\title{
Inflammation as orchestrator of cardiac disease progression
}

Citation for published version (APA):

Derks, W. (2016). Inflammation as orchestrator of cardiac disease progression. [Doctoral Thesis, Maastricht University]. https://doi.org/10.26481/dis.20160603wd

Document status and date:

Published: 01/01/2016

DOI:

10.26481/dis.20160603wd

Document Version:

Publisher's PDF, also known as Version of record

\section{Please check the document version of this publication:}

- A submitted manuscript is the version of the article upon submission and before peer-review. There can be important differences between the submitted version and the official published version of record.

People interested in the research are advised to contact the author for the final version of the publication, or visit the DOI to the publisher's website.

- The final author version and the galley proof are versions of the publication after peer review.

- The final published version features the final layout of the paper including the volume, issue and page numbers.

Link to publication

\footnotetext{
General rights rights.

- You may freely distribute the URL identifying the publication in the public portal. please follow below link for the End User Agreement:

www.umlib.nl/taverne-license

Take down policy

If you believe that this document breaches copyright please contact us at:

repository@maastrichtuniversity.nl

providing details and we will investigate your claim.
}

Copyright and moral rights for the publications made accessible in the public portal are retained by the authors and/or other copyright owners and it is a condition of accessing publications that users recognise and abide by the legal requirements associated with these

- Users may download and print one copy of any publication from the public portal for the purpose of private study or research.

- You may not further distribute the material or use it for any profit-making activity or commercial gain

If the publication is distributed under the terms of Article $25 \mathrm{fa}$ of the Dutch Copyright Act, indicated by the "Taverne" license above, 


\title{
Inflammation as orchestrator of cardiac disease progression
}

\author{
Wouter J.A. Derks
}


(C) Copyright Wouter Derks, Maastricht 2016

ISBN: 9789462953789

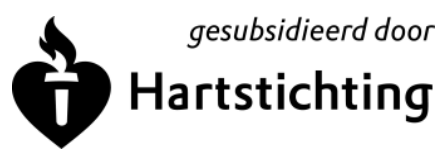

Financial support by the Dutch Heart Foundation for the publication of this thesis is gratefully acknowledged 


\title{
Inflammation as orchestrator of cardiac disease progression
}

\author{
PROEFSCHRIFT \\ Ter verkrijging van de graad doctor \\ aan de Universiteit Maastricht, \\ op gezag van de Rector Magnificus, \\ Prof. dr. L.L.G Soete, \\ volgens het besluit van het College van Decanen, \\ in het openbaar te verdedigen op \\ vrijdag 3 juni 2016 om 10.00 uur \\ door \\ Wouter Johannes Antonius Derks \\ Geboren op 5 mei 1984 te Venray
}




\section{Promotores}

Prof. dr. S. Heymans

Prof. dr. E. Lutgens (Universiteit van Amsterdam)

\section{Copromotor}

Dr. M. van Bilsen

\section{Beoordelingscommissie}

Prof. dr. C. Schalkwijk (Voorzitter)

Prof. dr. R.A. de Boer (Rijksuniversiteit Groningen)

Dr. P.A. da Costa Martins

Prof dr. F.W. Prinzen

Prof. dr. M.P.J. de Winther (Universiteit van Amsterdam) 


\section{Table of contents}

Chapter $1 \quad$ General introduction

Chapter 2 Inhibition of CD40-CD40 ligand (CD40L) and

downstream TRAF signalling reduces

angiotensin II induced cardiac hypertrophy

Chapter 3 Inflammation-induced metabolic remodelling

41

of the heart in acute viral myocarditis

Chapter 4 MicroRNAs act as pleiotropic regulators of

75

heart failure progression - A high-throughput

phenotypical screen in three primary cardiac cell types

Chapter $5 \quad$ Literature-based gene to phenotype interaction network

105

analysis reveals novel microRNA regulators in heart failure

Chapter 6 General discussion

Addendum: Summary/Samenvatting

159

Valorization/Valorisatie

Dankwoord

Curriculum Vitae

List of publications 
Chapter 1

\section{General introduction}

Inflammation as orchestrator of cardiac disease progression 


\section{Heart Failure}

Heart failure (HF) is the end stage of many cardiovascular diseases and is characterized by a decrease in cardiac function and by the inability of the heart to supply the body with adequate amounts of (oxygenated) blood. Patients suffer from shortness of breath, congestion and fatigue, which strongly reduces their quality of life. Its prevalence worldwide is high and is expanding in western society due to increased aging of the population and better survival of acute cardiovascular incidents. Currently about 1-2\% of the world's population suffers from HF and amongst elderly this is even $7-10 \%{ }^{1,2}$. Half of the people diagnosed with severe HF die within 5 years ${ }^{3,4}$. With this high prevalence, mortality rate and morbidity, HF is a huge burden on our society ${ }^{5}$. HF derives from a wide range of underlying disease aetiologies, such as a myocardial infarction (MI) or chronic hypertension but also diabetes and obesity ${ }^{6}$ or viral infections ${ }^{7}$ can be causes. Although HF treatment using digitalis has been around since the $16^{\text {th }}$ century, the high mortality and morbidity and the expanding prevalence show that there is a desperate need of improved therapies still.

Generally speaking, two types of HF can be distinguished, systolic and diastolic HF. Historically, HF was only suspected when systolic function was impaired and this systolic HF is characterized by a reduced fraction of blood leaving the left ventricle of the heart upon contraction (Ejection Fraction, EF). Normally between 50 and $70 \%$ of the blood leaves the left ventricle during systole but, in $\mathrm{HF}$ patients with systolic dysfunction this $\mathrm{EF}$ is reduced to less than $40 \%$. Patients suffering from diastolic HF, on the other hand, have a normal EF but the filling of the left ventricle during diastole is impaired. Both these types of HF cause similar symptoms in patients, but especially for the latter condition treatment option are limited ${ }^{8}$. For the development of novel treatment strategies for $\mathrm{HF}$, a more complete understanding of the processes underlying HF development and progression is crucial. The research described in this thesis aims to better understand these pathophysiological processes and the molecular mechanisms underlying them.

\section{Pathophysiology of HF and myocardial structural remodelling}

$\mathrm{HF}$ is a multifactorial disease that can develop rapidly, for instance after acute myocardial ischemia (AMI) or after viral infection of the cardiac tissue (viral myocarditis, VM). Alternatively, HF develops more gradually by sustained overloading of the heart, secondary to hypertension or valvular disease. The cardiac muscle tries to cope with this acute damage or chronic stress in various ways. These changes are commonly referred to as myocardial structural remodelling and include the enlargement of cardiomyocytes (hypertrophy), excessive deposition of extracellular matrix (fibrosis), and the infiltration of immune cells (inflammation) (Figure 1). Also crucial for the development of cardiac dysfunction are perturbations in cardiac energy metabolism during $\mathrm{HF}^{9}$.

The cardiac muscle contains multiple cell types (cardiomyocytes, fibroblasts, endothelial cells and resident immune cells) that become activated during pathophysiologic stress and contribute to structural remodelling in their own way. Generally speaking, cells are activated after ligand binding to a cell surface or intra-cellular receptor that leads to activation of intracellular signal-transduction pathways. Eventually, this results in the activation or translocation of transcription factors towards to nucleus, where they control gene expression. An exciting discovery was the identification of a class of small RNAs that negatively regulate gene expression and these, so-called microRNAs (miRNAs) ${ }^{10,11}$ add 
additional complexity to the regulation of gene/protein expression and have been shown to regulate pathophysiological remodelling processes associated with $\mathrm{HF}^{12-14}$.

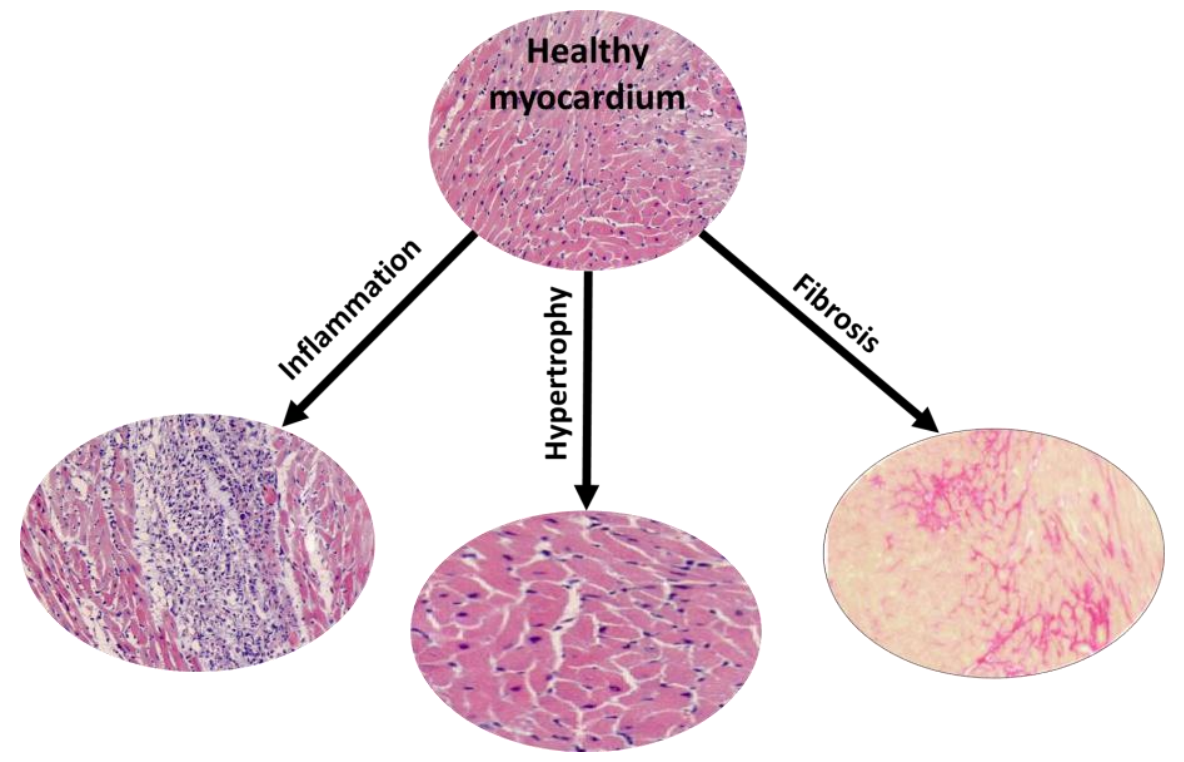

Figure 1. Histological analysis of the myocardium reveals the pathophysiological processes of cardiac inflammation, marked by the infiltration of immune cells, hypertrophy (enlargement of cardiomyocytes) and fibrosis (excessive collagen production). Cross-sections of healthy (above) and diseased (below) mouse hearts are stained with H\&E or Picro-Sirius Red.

\section{Cardiac hypertrophy}

Cardiac hypertrophy is an adaptive response of the heart to increased workload. It is characterized by enlargement of existing cardiomyocytes leading to ventricular wall thickening 15 and can either be pathological or physiological. Although short-term hypertrophic remodelling may be adaptive, chronic and continuous activation of this response is detrimental ${ }^{16-18}$. Actually, it is even debated if the development of hypertrophy is beneficial in the early stages of $\mathrm{HF}^{19}$, or if it is detrimental at any stage ${ }^{20}$. Interestingly, the heart can also develop a physiological hypertrophy in response to exercise (also known as athlete's heart) or pregnancy. There is evidence that these different types of hypertrophy are initiated via distinct molecular signalling pathways ${ }^{21}$. Stimuli for cardiac hypertrophy can be broadly segregated into biomechanical (stretch-sensitive) and neuro-humoral factors. Among the latter are Angiotensin II (AngII) and Endothelin-1 (ET-1). ET-1 is a paracrine regulator of cardiac hypertrophy secreted by endothelial cells in the microvasculature of the myocardium. In cultured cardiomyocytes of neonatal rats, stimulation with ET-1 induced hypertrophic responses characterized by an increase in protein synthesis and cell size and reactivation of the foetal gene program ${ }^{22}$. Angll induces cardiac hypertrophy mainly through its vasoconstrictive effects, leading to an increase in blood pressure ${ }^{16}$, but has also been shown to have a direct pro-hypertrophic effect on cardiomyocytes ${ }^{23}$.

\section{Cardiac fibrosis}

Another pathophysiological characteristic of many cardiac diseases is excessive myocardial fibrosis. During the process of cardiac remodelling, not only the cellular component of the myocardium undergoes changes but also the non-cellular part, called the extracellular matrix 
(ECM). Fibrosis is the adverse accumulation of collagens and other ECM proteins, which impairs ventricular function and predisposes the cardiac arrhythmias. Increased deposition of these collagens promotes abnormal myocardial stiffness that reduces the cardiac output ${ }^{24,25}$. During myocardial structural remodelling fibroblasts proliferate and differentiate into myofibroblasts, thereby secreting collagens ${ }^{26}$. Not only the amount of collagen changes in the diseased heart but also its composition, the degree of crosslinking, and thereby its quality 27. The final amount of fibrosis is always the result of a balance between collagen production and collagen degradation. Crucial for collagen degradation are the so-called matrix metalloproteinases (MMPs) that degrade collagen. MMPs in turn, are regulated by a family of tissue inhibitors of metalloproteinases (TIMPs) that counteract the effect of MMPs. The balance between MMP and TIMP activities is crucial for the amount of collagen in the heart. Transforming Growth Factor- $\beta$ (TGF $\beta$ ) is a pleiotropic cytokine expressed by multiple cell types and is a potent inducer of fibrosis ${ }^{28}$. TGF $\beta$ induces the expression of ECM proteins and suppresses the activity of genes encoding the MMPs. In addition, TGF $\beta$ induces the expression of Type I collagen and of pro-fibrotic genes such as connective tissue growth factor (CTGF) ${ }^{29}$. TGF $\beta$ has been shown to be upregulated in patients with $\mathrm{HF}^{30}$.

\section{Cardiac metabolism in HF}

The heart is one the most energy demanding organs of the body and at the same time has a limited capacity for storing fuel. The healthy heart derives most of its energy from the oxidation of fatty acids (FA) in a process called oxidative phosphorylation, that takes place inside the mitochondria ${ }^{9}$. This process efficiently generates energy in the form of ATP, required for the rhythmic cardiac muscle contraction. During $\mathrm{HF}$, perturbations in mitochondrial function have been observed ${ }^{9}$. In addition, a shift in cardiac metabolism away from the normal substrate preference of fatty acids towards glucose and towards glycolysis instead of oxidation takes place ${ }^{31}$. Ultimately, this leads to inefficient substrate use and ATP deficiency, a process referred to as "energy starvation". In this way cardiac metabolic remodelling contributes to HF progression ${ }^{32,33}$. Alterations in the expression of proteins involved in substrate uptake and conversion drive the cardiac metabolic remodelling process to a large extent. The transcriptional regulation of many genes involved in cardiac metabolism is under control of the Peroxisome proliferator-activated receptors (PPARs) and the estrogen related receptor- $\alpha(E R R \alpha){ }^{34,35}$. The transcriptional activities of these nuclear receptors are modulated by several co-repressor and co-activator proteins, among which the PPAR $\gamma$ coactivator- $1 \alpha$ (PGC- $1 \alpha$ ). PGC- $1 \alpha$ binds to and co-activates PPAR $\alpha$ and ERR $\alpha$, leading to increased capacity of FA uptake and oxidation. PGC-1 $\alpha$ is also a powerful initiator of mitochondrial biogenesis. During HF the expression and activation of these crucial regulators of metabolism is reduced.

\section{Inflammation at the heart of structural remodelling?}

Damage to the myocardium causes cell death and stress that subsequently initiates an inflammatory response. Necrotic, apoptotic or stressed cells secrete factors that can be recognized by other cells as Damage Associated Molecular Patterns (DAMPS) ${ }^{36}$. Also pathogens present in the heart can be recognized by cells in a similar way, these are referred to as Pathogen Associated Molecular Patterns (PAMPs). Almost all cell types have specific cell surface pattern recognition receptors (PRRs) for these PAMPs and DAMPS of which the family of Toll-like receptors (TLRs) is the most prominent. Activation of these receptors initiates intra-cellular pathways that result in the expression of pro-inflammatory mediators. The NFKB pathway is the main pathway involved here and controls expression of a variety of 
pro-inflammatory cytokines, such as TNF $\alpha$. In patients with HF it has been observed that a network of pro-inflammatory mediators (e.g., TNF $\alpha$, interleukin-1 $\beta$, interleukin-6) are expressed ${ }^{37}$ and inflammation has been shown to have crucial effects on all of the pathophysiological processes described previously ${ }^{38}$. Pro-inflammatory effectors of the immune system increase pathophysiological stress. In patients with $\mathrm{HF}$, the concentration of the pro-inflammatory cytokines TNF $\alpha$, IL-6, and IL-1 $\beta$ correlates with disease severity ${ }^{39}$. TNF $\alpha$ is an important pro-inflammatory cytokine and exerts strong effects on all cell types present in the heart ${ }^{40}$. Ligation to its receptor (TNFR1) results in activation of the classical NFKB Pathway, creating a positive feedback mechanism that increases the inflammatory response. Next to the expression of cytokines, activation of the pro-inflammatory pathways via NFKB also leads to the secretion of specific type of chemotactic cytokines (chemokines) that attract leukocytes from the blood into the tissue. One of these chemokines, MCP-1 (or CCL2) is the major factor responsible for attracting monocytes into the myocardium. Following infiltration these monocytes differentiate into macrophages. These macrophages in turn can polarise into several phenotypes that can either be pro- or anti-inflammatory. Macrophages stimulated by TLR ligands and interferon (IFN) $-\gamma$ undergo classical M1 activation, while those stimulated by the anti-inflammatory cytokine IL-4 become $\mathrm{M} 2$ macrophages ${ }^{41}$. M1 macrophages exert acute pro-inflammatory effects, such as the secretion of MMPs and NO, and can be phagocytic and proteolytic. M2 macrophages, on the other hand, are involved in wound healing, tissue remodelling and induce fibrosis. In recent history the cardiac immune response has been studied in pre-clinical models ${ }^{38}$. It became clear that TNF $\alpha$ had adverse effects in the hearts of both mice ${ }^{42}$ and rats ${ }^{43}$ and that deletion of TNF $\alpha$ reduced HF pathology in these rodents ${ }^{44}$. Already more than 10 years ago the first clinical trial targeting TNF $\alpha$ in HF patients was performed, using a drug already approved for the treatment of rheumatoid arthritis. The results of this trial proved disappointing as TNF $\alpha$ blockade even led to a worsened clinical outcome in HF patients ${ }^{45}$. This indicated that the exact role of inflammation and the immune system in the disease progression of HF cannot be generalised and that individual effectors of the immune system such as cytokines and chemokines can have either beneficial or detrimental effects depending on the tissue, cell type, disease progression and the underlying HF aetiology. Beneficial processes orchestrated by the immune system are wound healing, debris clearance and pathogen clearance. Numerous immune and non-immune cell types are involved in orchestrating this pathological response. Researchers have yet only started to solve this puzzle, since these responses are complex, depend on the cell type, and are mostly characterized only in vitro. Furthermore, the cell types producing these cytokines have not been identified in many cases, and even the target cells of most cytokines have not been fully defined in vivo. The complexity is further increased by the temporal changes that occur in the injury and immune responses, leading to an incomplete understanding of the role of these cytokines during disease. Nevertheless, some interesting discoveries have been made recently concerning the effect of individual effectors of the immune system on cardiovascular disease progression. Manipulation of the immune response was proven to successfully prevent progression of HF. For instance, adoptive transfer of immunosuppressive T-regulatory cells ${ }^{46}$ and polarisation of macrophages towards a more anti-inflammatory phenotype attenuated both cardiac hypertrophy and dysfunction 47,48 . 


\section{Inflammation and cardiac hypertrophy}

There are numerous examples of studies where blocking the signalling of pro-inflammatory cytokines reduces cardiac hypertrophy ${ }^{49}{ }^{50}$. In the heart, cardiac macrophages participate in the development of cardiac hypertrophy ${ }^{51}$ by secreting cytokines and growth factors like the pro-hypertrophic IL-6. Macrophages polarised towards M1 are known to increase cardiac hypertrophy and failure after cardiac PO ${ }^{47}, 48$. Both cardiomyocyte and non-cardiomyocyte cells secrete and respond to numerous cytokines, several pro-inflammatory pathways have been shown to modulate cardiac hypertrophy upon PO in mice, including TNF Receptor $1^{52}$ and TNF itself ${ }^{44}$, MCP-1 5349 and IL-6 ${ }^{54} 50$.

\section{Inflammation and cardiac fibrosis}

Inflammation has crucial effects on the balance between the production and degradation of collagens ${ }^{55}$. Tissue infiltrating pro-inflammatory immune cells, like M1 macrophages and neutrophils secrete granules containing MMPs and other factors capable of degrading collagen and other connective tissue components of the ECM ${ }^{56}$. This process facilitates their migration into inflamed tissues. Macrophages are also important effector cells in tissue remodelling and fibrosis and are a main source of MMPs (MMP1-12) as well as their suppressors, the TIMPs. Alternatively activated (M2) macrophages are also a major source of TGF $\beta$, considered the most pro-fibrotic agent. On the other hand, TGF $\beta$ has potent antiinflammatory effects, including the induction regulatory T-cells ${ }^{57}$, and stimulates fibrosis at the same time. These pleiotropic effects on inflammation and fibrosis are clearly shown in TGF $\beta$-deficient mice that exhibit profoundly reduced collagen deposition, while suffering from extensive inflammation and tissue necrosis ${ }^{58,59}$. In the murine heart inhibition of TGF $\beta$ increased leukocyte infiltration and chemokine expression after $\mathrm{MI}{ }^{60}$. Fibroblasts themselves also express PRRs, including the TLRs, and DAMPs activating these receptors can directly promote fibroblast differentiation into collagen-producing myofibroblasts ${ }^{61}$.

\section{Inflammation and cardiac metabolism}

Metabolic stress is an important inducer of cardiac inflammation. Inflammatory pathways are activated during cardiomyocyte hypertrophy and are attenuated by peroxisome proliferatoractivated receptors PPAR $\alpha$ and PPAR ${ }^{62,63}$. Reactive oxygen species (ROS) from stressed mitochondria, that are considered DAMPs, have an established role in inflammation and host defence, as they kill intracellular bacteria and have been shown to initiate inflammatory responses. ROS generated during mitochondrial respiration induces the production of proinflammatory cytokines. Oka et al. ${ }^{64}$ demonstrated in mice with aortic constriction that mitochondrial DNA that escapes from autophagy causes cardiac inflammation and failure upon recognition by a specific type of TLR (TLR9) that detects extracellular DNA. The polarisation status of macrophages determines their metabolic phenotype ${ }^{65}$. M1 polarised macrophages display a metabolic shift towards anaerobic glycolysis. In contrast, IL-4activated M2 mouse macrophages show a significant upregulation of mitochondrial fatty acid oxidation ${ }^{66}$. Apart from metabolic stress inducing cardiac inflammation the inverse has also been shown, in that NFKB activation induces metabolic remodelling away from oxidative processes towards a greater reliance on glycolytic metabolism ${ }^{63,67}$.

\section{Inflammation in acute VM and chronic HHD}

Acute HF and chronic HF are characterised by distinct inflammatory mechanisms and in this thesis two different types of HF aetiologies were studied, namely, Hypertensive Heart Disease (HHD) and Viral Myocarditis (VM). HHD which is associated with chronic, low-grade 
inflammation. HHD causes pressure overloading (PO) of the heart and ultimately leads to HF. In western society it is most prevalent amongst elderly woman. In the corresponding mouse model of HHD, hypertension is induced via infusion of Angll using implanted osmotic minipumps. VM is characterized by an acute and fulminant inflammatory response, and VM is an important cause of cardiovascular death in young, otherwise healthy individuals often as a result of common cough/influenza viruses ${ }^{68}$. In a subgroup of patients, VM gives rise to an autoimmune response predisposing to the development of dilated cardiomyopathy (DCM) ${ }^{7}$, ${ }^{69}$. In the mouse model of this disease, myocarditis in induced by coxsackievirus B3 (CVB3). Most mouse strains are not susceptible to develop CVB3 induced VM, only specific strains, like $\mathrm{C} 3 \mathrm{H}$ or Balb/c mice, develop myocarditis upon CVB3 infection, this is most likely due to differences in immuno-genetic background ${ }^{70}$.

\section{Aims and outline of this thesis}

The general aim of this thesis is to understand the role of inflammation in HF and to explore how it can be manipulated in such way that it preserves its crucial role in beneficial processes such as wound healing, debris clearance and pathogen clearance, but that its destructive effects on the cardiac tissue are diminished. To this aim, we have used patient material, different animal models and in vitro and in silico screening approaches.

Chapter two focusses on the deletion and manipulation of the pro-inflammatory CD40 receptor in an animal model of hypertensive induced HF. CD40 (or TNFRSF5) is a cellsurface receptor belonging to the tumor necrosis factor (TNF) receptor super family and is predominantly expressed by antigen-presenting cells, and its ligation with its ligand-receptor CD40L is a crucial step in the process of antigen presentation. As an important effector of the immune system, the CD40 receptor has been proven to be a critical regulator of the immune response in different chronic inflammatory diseases like atherosclerosis, multiple sclerosis and arthritis ${ }^{71-73}$. In all of these pathologies the blocking of CD40L-CD40 interactions was found to reduce disease severity. The effect of this receptor on the development of HF, as induced by chronic hypertension, has never been investigated before.

In chapter three it is investigated how the acute and fulminant inflammatory response affects cardiac metabolism in VM patients and in a mouse model of CVB3-induced VM. The acute effects of pronounced cardiac inflammation, and VM in particular, on cardiac metabolism are not well studied so far. For this extensive characterisation we made use of cardiac samples of VM patients and the CVB3 mouse VM model. We hypothesize that the fulminant inflammatory response associated with acute VM disturbs cardiomyocyte energy metabolism, thereby compromising cardiac energy metabolism. In addition, it was explored if the manipulation of cardiac metabolism has therapeutic potential.

Over the last decade high-throughput screening has developed into an indispensable tool to identify new therapeutic molecules ${ }^{74}$. In chapter four an in vitro highthroughput screening approach is applied in which 194 miRNAs, previously identified to be differentially expressed in inflammatory diseases of the heart, were tested for their effects on hypertrophy, fibrosis and inflammation in different cell types. The miRNA field is developing rapidly and the manipulation of their activity via mimics or anti-miRs holds great therapeutic promise ${ }^{13,75}$. By transfecting unstimulated and stimulated cardiomyocytes, fibroblasts and bone marrow-derived macrophages (BMDM) with miRNA mimics, we identified novel roles for many miRNAs in the cardiac remodelling process. This research was performed at Cenix bioscience, Dresden, Germany, a company that was renowned internationally for its expertise in high-throughput screening. 
In chapter five of this thesis an extensive overview was made of preclinical studies dealing with inflammation in cardiac PO. To this end, we identified articles that studied and manipulated cardiac inflammation, fibrosis and hypertrophy upon PO. From almost 300 articles the data was extracted. The dataset obtained was used to define and compare the features of different rodent models of PO and served as input for network analysis, linking phenotypical outcomes to genes and, subsequently to miRNAs that target these genes

Finally, in chapter six the findings of the previous chapters are summarized and discussed in broader perspective. 


\section{References}

1. McMurray JJ, Adamopoulos S, Anker SD, Auricchio A, Bohm M, Dickstein K, Falk V, Filippatos G, Fonseca C, Gomez-Sanchez MA, Jaarsma T, Kober L, Lip GY, Maggioni AP, Parkhomenko A, Pieske BM, Popescu BA, Ronnevik PK, Rutten FH, Schwitter J, Seferovic P, Stepinska J, Trindade PT, Voors AA, Zannad F, Zeiher A, Bax JJ, Baumgartner H, Ceconi C, Dean V, Deaton C, Fagard R, Funck-Brentano C, Hasdai D, Hoes A, Kirchhof P, Knuuti J, Kolh P, McDonagh T, Moulin C, Popescu BA, Reiner Z, Sechtem U, Sirnes PA, Tendera $M$, Torbicki A, Vahanian A, Windecker S, McDonagh T, Sechtem U, Bonet LA, Avraamides $P$, Ben Lamin HA, Brignole M, Coca A, Cowburn P, Dargie H, Elliott P, Flachskampf FA, Guida GF, Hardman S, lung B, Merkely B, Mueller C, Nanas JN, Nielsen OW, Orn S, Parissis JT, Ponikowski P and Guidelines ESCCfP. ESC guidelines for the diagnosis and treatment of acute and chronic heart failure 2012: The Task Force for the Diagnosis and Treatment of Acute and Chronic Heart Failure 2012 of the European Society of Cardiology. Developed in collaboration with the Heart Failure Association (HFA) of the ESC. Eur J Heart Fail. 2012;14:803-69.

2. Burchfield JS, Xie M and Hill JA. Pathological ventricular remodeling: mechanisms: part 1 of 2. Circulation. 2013;128:388-400.

3. Bhatia RS, Tu JV, Lee DS, Austin PC, Fang J, Haouzi A, Gong Y and Liu PP. Outcome of heart failure with preserved ejection fraction in a population-based study. N Engl J Med. 2006;355:260-9.

4. Bleumink GS, Knetsch AM, Sturkenboom MC, Straus SM, Hofman A, Deckers JW, Witteman JC and Stricker BH. Quantifying the heart failure epidemic: prevalence, incidence rate, lifetime risk and prognosis of heart failure The Rotterdam Study. Eur Heart J. 2004;25:1614-9.

5. Lloyd-Jones D, Adams RJ, Brown TM, Carnethon M, Dai S, De Simone G, Ferguson TB, Ford E, Furie K, Gillespie C, Go A, Greenlund K, Haase N, Hailpern S, Ho PM, Howard V, Kissela B, Kittner S, Lackland D, Lisabeth L, Marelli A, McDermott MM, Meigs J, Mozaffarian D, Mussolino M, Nichol G, Roger VL, Rosamond W, Sacco R, Sorlie P, Stafford R, Thom T, Wasserthiel-Smoller S, Wong ND, Wylie-Rosett J, American Heart Association Statistics $C$ and Stroke Statistics S. Executive summary: heart disease and stroke statistics--2010 update: a report from the American Heart Association. Circulation. 2010;121:94854.

6. Miki T, Yuda S, Kouzu H and Miura T. Diabetic cardiomyopathy: pathophysiology and clinical features. Heart Fail Rev. 2013;18:149-66.

7. Dennert R, Crijns HJ and Heymans S. Acute viral myocarditis. Eur Heart J. 2008;29:2073-82.

8. Rutten FH, Cramer MJ and Paulus WJ. [Heart failure with preserved ejection fraction: Diastolic heart failure]. Ned Tijdschr Geneeskd. 2012;156:A5315.

9. Doenst T, Nguyen TD and Abel ED. Cardiac metabolism in heart failure: implications beyond ATP production. Circ Res. 2013;113:709-24.

10. Novina CD and Sharp PA. The RNAi revolution. Nature. 2004;430:161-4.

11. Ha M and Kim VN. Regulation of microRNA biogenesis. Nat Rev Mol Cell Biol. 2014;15:509-24.

12. Ikeda S, He A, Kong SW, Lu J, Bejar R, Bodyak N, Lee KH, Ma Q, Kang PM, Golub TR and Pu WT. MicroRNA1 negatively regulates expression of the hypertrophy-associated calmodulin and Mef2a genes. Mol Cell Biol. 2009;29:2193-204.

13. van Rooij E, Sutherland LB, Thatcher JE, DiMaio JM, Naseem RH, Marshall WS, Hill JA and Olson EN. Dysregulation of microRNAs after myocardial infarction reveals a role of miR-29 in cardiac fibrosis. Proc Natl Acad Sci U S A. 2008;105:13027-32.

14. Corsten MF, Papageorgiou A, Verhesen W, Carai P, Lindow M, Obad S, Summer G, Coort SL, Hazebroek M, van Leeuwen R, Gijbels MJ, Wijnands E, Biessen EA, De Winther MP, Stassen FR, Carmeliet P, Kauppinen S, Schroen B and Heymans S. MicroRNA profiling identifies microRNA-155 as an adverse mediator of cardiac injury and dysfunction during acute viral myocarditis. Circulation research. 2012;111:415-25.

15. Grossman W, Jones D and McLaurin LP. Wall stress and patterns of hypertrophy in the human left ventricle. J Clin Invest. 1975;56:56-64.

16. Unger T. The role of the renin-angiotensin system in the development of cardiovascular disease. Am $\mathrm{J}$ Cardiol. 2002;89:3A-9A; discussion 10A.

17. Paradis P, Dali-Youcef N, Paradis FW, Thibault G and Nemer M. Overexpression of angiotensin II type I receptor in cardiomyocytes induces cardiac hypertrophy and remodeling. Proc Natl Acad Sci U S A. 2000;97:931-6.

18. Levy D, Garrison RJ, Savage DD, Kannel WB and Castelli WP. Prognostic implications of echocardiographically determined left ventricular mass in the Framingham Heart Study. $N$ Engl J Med. 1990;322:1561-6. 
19. Crozatier B and Ventura-Clapier R. Inhibition of hypertrophy, per se, may not be a good therapeutic strategy in ventricular pressure overload: other approaches could be more beneficial. Circulation. 2015;131:1448-57.

20. Schiattarella GG and Hill JA. Inhibition of hypertrophy is a good therapeutic strategy in ventricular pressure overload. Circulation. 2015;131:1435-47.

21. Mudd JO and Kass DA. Tackling heart failure in the twenty-first century. Nature. 2008;451:919-28.

22. Ito $\mathrm{H}$, Hirata $\mathrm{Y}$, Hiroe $\mathrm{M}$, Tsujino $\mathrm{M}$, Adachi S, Takamoto $\mathrm{T}$, Nitta M, Taniguchi $\mathrm{K}$ and Marumo $\mathrm{F}$. Endothelin-1 induces hypertrophy with enhanced expression of muscle-specific genes in cultured neonatal rat cardiomyocytes. Circ Res. 1991;69:209-15.

23. Watkins SJ, Borthwick GM, Oakenfull R, Robson A and Arthur HM. Angiotensin II-induced cardiomyocyte hypertrophy in vitro is TAK1-dependent and Smad2/3-independent. Hypertens Res. 2012;35:393-8.

24. Watson CJ, Phelan D, Xu M, Collier P, Neary R, Smolenski A, Ledwidge M, McDonald K and Baugh J. Mechanical stretch up-regulates the B-type natriuretic peptide system in human cardiac fibroblasts: a possible defense against transforming growth factor-beta mediated fibrosis. Fibrogenesis \& tissue repair. 2012;5:9.

25. Booz GW and Baker KM. Molecular signalling mechanisms controlling growth and function of cardiac fibroblasts. Cardiovasc Res. 1995;30:537-43.

26. Assomull RG, Prasad SK, Lyne J, Smith G, Burman ED, Khan M, Sheppard MN, Poole-Wilson PA and Pennell DJ. Cardiovascular magnetic resonance, fibrosis, and prognosis in dilated cardiomyopathy. J Am Coll Cardiol. 2006;48:1977-85.

27. Lopez B, Querejeta R, Gonzalez A, Larman M and Diez J. Collagen cross-linking but not collagen amount associates with elevated filling pressures in hypertensive patients with stage $C$ heart failure: potential role of lysyl oxidase. Hypertension. 2012;60:677-83.

28. Bronnum H, Eskildsen T, Andersen DC, Schneider M and Sheikh SP. IL-1beta suppresses TGF-betamediated myofibroblast differentiation in cardiac fibroblasts. Growth Factors. 2013;31:81-9.

29. Ruiz-Ortega M, Rodriguez-Vita J, Sanchez-Lopez E, Carvajal G and Egido J. TGF-beta signaling in vascular fibrosis. Cardiovasc Res. 2007;74:196-206.

30. Westermann D, Lindner D, Kasner M, Zietsch C, Savvatis K, Escher F, von Schlippenbach J, Skurk C, Steendijk P, Riad A, Poller W, Schultheiss HP and Tschope C. Cardiac inflammation contributes to changes in the extracellular matrix in patients with heart failure and normal ejection fraction. Circulation Heart failure. 2011;4:44-52.

31. Davila-Roman VG, Vedala G, Herrero P, de las Fuentes L, Rogers JG, Kelly DP and Gropler RJ. Altered myocardial fatty acid and glucose metabolism in idiopathic dilated cardiomyopathy. J Am Coll Cardiol. 2002;40:271-7.

32. van Bilsen $M$, van Nieuwenhoven FA and van der Vusse GJ. Metabolic remodelling of the failing heart: beneficial or detrimental? Cardiovascular research. 2009;81:420-8.

33. Kolwicz SC, Jr., Purohit S and Tian R. Cardiac metabolism and its interactions with contraction, growth, and survival of cardiomyocytes. Circ Res. 2013;113:603-16.

34. van Bilsen $\mathrm{M}$ and van Nieuwenhoven FA. PPARs as therapeutic targets in cardiovascular disease. Expert Opin Ther Targets. 2010;14:1029-45.

35. Huss JM, Garbacz WG and Xie W. Constitutive activities of estrogen-related receptors: Transcriptional regulation of metabolism by the ERR pathways in health and disease. Biochim Biophys Acta. 2015;1852:1912-27.

36. Seong SY and Matzinger P. Hydrophobicity: an ancient damage-associated molecular pattern that initiates innate immune responses. Nat Rev Immunol. 2004;4:469-78.

37. Aukrust P, Ueland T, Lien E, Bendtzen K, Muller F, Andreassen AK, Nordoy I, Aass H, Espevik T, Simonsen S, Froland SS and Gullestad L. Cytokine network in congestive heart failure secondary to ischemic or idiopathic dilated cardiomyopathy. Am J Cardiol. 1999;83:376-82.

38. Frieler RA and Mortensen RM. Immune cell and other noncardiomyocyte regulation of cardiac hypertrophy and remodeling. Circulation. 2015;131:1019-30.

39. Testa M, Yeh M, Lee P, Fanelli R, Loperfido F, Berman JW and LeJemtel TH. Circulating levels of cytokines and their endogenous modulators in patients with mild to severe congestive heart failure due to coronary artery disease or hypertension. J Am Coll Cardiol. 1996;28:964-71.

40. Gullestad L, Ueland T, Vinge LE, Finsen A, Yndestad A and Aukrust P. Inflammatory cytokines in heart failure: mediators and markers. Cardiology. 2012;122:23-35.

41. Murray PJ, Allen JE, Biswas SK, Fisher EA, Gilroy DW, Goerdt S, Gordon S, Hamilton JA, Ivashkiv LB, Lawrence T, Locati M, Mantovani A, Martinez FO, Mege JL, Mosser DM, Natoli G, Saeij JP, Schultze JL, Shirey KA, Sica A, Suttles J, Udalova I, van Ginderachter JA, Vogel SN and Wynn TA. Macrophage activation and polarization: nomenclature and experimental guidelines. Immunity. 2014;41:14-20. 
42. Kubota T, McTiernan CF, Frye CS, Slawson SE, Lemster BH, Koretsky AP, Demetris AJ and Feldman AM. Dilated cardiomyopathy in transgenic mice with cardiac-specific overexpression of tumor necrosis factoralpha. Circ Res. 1997;81:627-35.

43. Bozkurt B, Kribbs SB, Clubb FJ, Jr., Michael LH, Didenko VV, Hornsby PJ, Seta Y, Oral H, Spinale FG and Mann DL. Pathophysiologically relevant concentrations of tumor necrosis factor-alpha promote progressive left ventricular dysfunction and remodeling in rats. Circulation. 1998;97:1382-91.

44. Sun M, Chen M, Dawood F, Zurawska U, Li JY, Parker T, Kassiri Z, Kirshenbaum LA, Arnold M, Khokha R and Liu PP. Tumor necrosis factor-alpha mediates cardiac remodeling and ventricular dysfunction after pressure overload state. Circulation. 2007;115:1398-407.

45. Mann DL, McMurray JJ, Packer M, Swedberg K, Borer JS, Colucci WS, Djian J, Drexler H, Feldman A, Kober L, Krum H, Liu P, Nieminen M, Tavazzi L, van Veldhuisen DJ, Waldenstrom A, Warren M, Westheim A, Zannad $\mathrm{F}$ and Fleming $\mathrm{T}$. Targeted anticytokine therapy in patients with chronic heart failure: results of the Randomized Etanercept Worldwide Evaluation (RENEWAL). Circulation. 2004;109:1594-602.

46. Kvakan H, Kleinewietfeld M, Qadri F, Park JK, Fischer R, Schwarz I, Rahn HP, Plehm R, Wellner M, Elitok S, Gratze P, Dechend R, Luft FC and Muller DN. Regulatory T cells ameliorate angiotensin II-induced cardiac damage. Circulation. 2009;119:2904-12.

47. Usher MG, Duan SZ, Ivaschenko CY, Frieler RA, Berger S, Schutz G, Lumeng CN and Mortensen RM. Myeloid mineralocorticoid receptor controls macrophage polarization and cardiovascular hypertrophy and remodeling in mice. J Clin Invest. 2010;120:3350-64.

48. Heymans S, Corsten MF, Verhesen W, Carai P, van Leeuwen RE, Custers K, Peters T, Hazebroek M, Stoger L, Wijnands E, Janssen BJ, Creemers EE, Pinto YM, Grimm D, Schurmann N, Vigorito E, Thum T, Stassen F, Yin X, Mayr M, de Windt LJ, Lutgens E, Wouters K, de Winther MP, Zacchigna S, Giacca M, van Bilsen M, Papageorgiou AP and Schroen B. Macrophage microRNA-155 promotes cardiac hypertrophy and failure. Circulation. 2013;128:1420-32.

49. Kuwahara F, Kai H, Tokuda K, Takeya M, Takeshita A, Egashira K and Imaizumi T. Hypertensive myocardial fibrosis and diastolic dysfunction: another model of inflammation? Hypertension. 2004;43:739-45.

50. Coles B, Fielding CA, Rose-John S, Scheller J, Jones SA and O'Donnell VB. Classic interleukin-6 receptor signaling and interleukin- 6 trans-signaling differentially control angiotensin II-dependent hypertension, cardiac signal transducer and activator of transcription-3 activation, and vascular hypertrophy in vivo. The American journal of pathology. 2007;171:315-25.

51. Kamo T, Akazawa $\mathrm{H}$ and Komuro I. Cardiac nonmyocytes in the hub of cardiac hypertrophy. Circ Res. 2015;117:89-98.

52. Duerrschmid C, Crawford JR, Reineke E, Taffet GE, Trial J, Entman ML and Haudek SB. TNF receptor 1 signaling is critically involved in mediating angiotensin-ll-induced cardiac fibrosis. J Mol Cell Cardiol. 2013;57:59-67.

53. Haudek SB, Cheng J, Du J, Wang Y, Hermosillo-Rodriguez J, Trial J, Taffet GE and Entman ML. Monocytic fibroblast precursors mediate fibrosis in angiotensin-II-induced cardiac hypertrophy. J Mol Cell Cardiol. 2010;49:499-507.

54. Hirota H, Yoshida K, Kishimoto T and Taga T. Continuous activation of gp130, a signal-transducing receptor component for interleukin 6-related cytokines, causes myocardial hypertrophy in mice. Proc Natl Acad Sci U S A. 1995;92:4862-6.

55. Van Linthout S, Miteva K and Tschope C. Crosstalk between fibroblasts and inflammatory cells. Cardiovasc Res. 2014;102:258-69.

56. Ma Y, Yabluchanskiy A and Lindsey ML. Neutrophil roles in left ventricular remodeling following myocardial infarction. Fibrogenesis Tissue Repair. 2013;6:11.

57. Huber S, Schramm C, Lehr HA, Mann A, Schmitt S, Becker C, Protschka M, Galle PR, Neurath MF and Blessing $M$. Cutting edge: TGF-beta signaling is required for the in vivo expansion and immunosuppressive capacity of regulatory CD4+CD25+ T cells. J Immunol. 2004;173:6526-31.

58. Kulkarni $A B$ and Karlsson S. Transforming growth factor-beta 1 knockout mice. A mutation in one cytokine gene causes a dramatic inflammatory disease. Am J Pathol. 1993;143:3-9.

59. Bottinger EP, Letterio JJ and Roberts AB. Biology of TGF-beta in knockout and transgenic mouse models. Kidney Int. 1997;51:1355-60.

60. Ikeuchi M, Tsutsui H, Shiomi T, Matsusaka H, Matsushima S, Wen J, Kubota T and Takeshita A. Inhibition of TGF-beta signaling exacerbates early cardiac dysfunction but prevents late remodeling after infarction. Cardiovasc Res. 2004;64:526-35.

61. Meneghin A and Hogaboam CM. Infectious disease, the innate immune response, and fibrosis. $J$ Clin Invest. 2007;117:530-8.

62. Smeets PJ, Planavila A, van der Vusse GJ and van Bilsen M. Peroxisome proliferator-activated receptors and inflammation: take it to heart. Acta Physiol (Oxf). 2007;191:171-88. 
63. Smeets PJ, Teunissen BE, Planavila A, de Vogel-van den Bosch H, Willemsen PH, van der Vusse GJ and van Bilsen $M$. Inflammatory pathways are activated during cardiomyocyte hypertrophy and attenuated by peroxisome proliferator-activated receptors PPARalpha and PPARdelta. J Biol Chem. 2008;283:29109-18.

64. Oka T, Hikoso S, Yamaguchi O, Taneike M, Takeda T, Tamai T, Oyabu J, Murakawa T, Nakayama H, Nishida K, Akira S, Yamamoto A, Komuro I and Otsu K. Mitochondrial DNA that escapes from autophagy causes inflammation and heart failure. Nature. 2012;485:251-5.

65. Biswas SK and Mantovani A. Orchestration of metabolism by macrophages. Cell Metab. 2012;15:432-7.

66. Odegaard $\mathrm{JI}$ and Chawla A. Alternative macrophage activation and metabolism. Annu Rev Pathol. 2011;6:275-97.

67. Remels AH, Gosker HR, Bakker J, Guttridge DC, Schols AM and Langen RC. Regulation of skeletal muscle oxidative phenotype by classical NF-kappaB signalling. Biochim Biophys Acta. 2013;1832:1313-25.

68. Blauwet LA and Cooper LT. Myocarditis. Prog Cardiovasc Dis. 2010;52:274-88.

69. Esfandiarei M and McManus BM. Molecular biology and pathogenesis of viral myocarditis. Annu Rev Pathol. 2008;3:127-55.

70. Corsten MF, Schroen B and Heymans S. Inflammation in viral myocarditis: friend or foe? Trends Mol Med. 2012;18:426-37.

71. Lutgens E, Gorelik L, Daemen MJ, de Muinck ED, Grewal IS, Koteliansky VE and Flavell RA. Requirement for CD154 in the progression of atherosclerosis. Nat Med. 1999;5:1313-6.

72. Gerritse K, Laman JD, Noelle RJ, Aruffo A, Ledbetter JA, Boersma WJ and Claassen E. CD40-CD40 ligand interactions in experimental allergic encephalomyelitis and multiple sclerosis. Proceedings of the National Academy of Sciences of the United States of America. 1996;93:2499-504.

73. Durie FH, Fava RA, Foy TM, Aruffo A, Ledbetter JA and Noelle RJ. Prevention of collagen-induced arthritis with an antibody to gp39, the ligand for CD40. Science. 1993;261:1328-30.

74. Echeverri CJ and Perrimon N. High-throughput RNAi screening in cultured cells: a user's guide. Nat Rev Genet. 2006;7:373-84.

75. van Rooij E and Olson EN. MicroRNA therapeutics for cardiovascular disease: opportunities and obstacles. Nature reviews Drug discovery. 2012;11:860-72. 


\section{Chapter 2}

\section{Inhibition of CD40-CD40 ligand (CD40L) and downstream TRAF signalling reduces angiotensin II induced cardiac hypertrophy}

\section{Authors:}

Wouter Derks ${ }^{1}$

Marc van Bilsen ${ }^{1,2}$

Paolo Carai ${ }^{1,3}$

Louis Boon 4

Esther Lutgens ${ }^{5}$

Stephane Heymans ${ }^{1,3}$

1.) Department of Cardiology. Cardiovascular Research Institute Maastricht (CARIM), Maastricht University, Maastricht, the Netherlands.

2.) Department of Physiology, Cardiovascular Research Institute Maastricht (CARIM), Maastricht University, Maastricht, the Netherlands.

3.) Center for Molecular and Cardiovascular Biology, Department of Cardiovascular Sciences, Leuven, Belgium.

4.) Bioceros, Utrecht, the Netherlands.

5.) Department of Medical Biochemistry, Academic Medical Centre, University of Amsterdam, Amsterdam, the Netherlands.

\section{Submitted to Clinical Science}




\begin{abstract}
Inflammation is emerging as a crucial modulator of hypertensive heart disease (HHD). Activation of the co-stimulatory CD40 receptor exerts pro-inflammatory effects via macrophages, and serum levels of soluble CD40-ligand are elevated in patients with HHD induced heart failure (HF). Here, we investigated whether CD40-CD40L interactions affect cardiac hypertrophy during HHD and which downstream TNF-receptor associated factor (TRAF) signalling molecules are involved. CD40 knockout (CD40 ${ }^{-/}$) and wild-type (Wt) mice were treated with Angiotensin II (Angll, $2.5 \mathrm{mg} / \mathrm{kg} /$ day) for 4 weeks. Ventricular mass increased significantly in $\mathrm{Wt}(+31 \%, \mathrm{P}<0.01)$, but not in $\mathrm{CD} 40^{-/-}$mice $(+10 \%, \mathrm{~N} . \mathrm{S}$.) upon Angll treatment. In line, the increase in diastolic LV wall thickness and cardiomyocyte cross sectional area was blunted in $\mathrm{CD} 40^{-/}$mice. To determine the CD40-TRAF interactions involved, the effect of site directed mutagenesis in CD40-TRAF binding on cardiac hypertrophy was investigated. Overexpression of intact CD40 in antigen presenting cells exacerbated cardiac hypertrophy upon pressure overload and relative to mice with intact downstream CD40 signalling, disruption of TRAF2/3/5, TRAF6 or both binding sites resulted in significantly reduced hypertrophy (Ventricular mass $-24 \%$ for CD40-T $\Delta 2,-28 \%$ for CD40$\mathrm{T} \Delta 6$ and $-26 \%$ in $\mathrm{CD} 40-\mathrm{T} \Delta 2 / 6 ; \mathrm{p}<0.05)$. Administration of $\mathrm{CD}_{40} /-$ macrophage-conditioned medium to cardiomyocytes in vitro blunted the hypertrophic response indicating that the secretome of $\mathrm{CD}_{40} 0^{--}$macrophages affects myocyte hypertrophy. In conclusion, inhibition of CD40 in myeloid cells decreases Angll-induced cardiac hypertrophy with direct involvement of the CD40-TRAF2/3/5 and CD40-TRAF6 pathways.
\end{abstract}




\section{Introduction}

The increasing prevalence of heart failure (HF) poses enormous challenges for health care systems worldwide. Hypertension triggers hypertrophic remodelling of the cardiac muscle, ultimately leading to the development of HF. Despite effective medical interventions mortality and morbidity remain substantial. Emerging evidence indicates that the activation of inflammatory processes plays a pivotal role in disease progression during hypertensive heart disease (HHD) ${ }^{1}$. The immune system favours HHD by the release of cytokines stimulating the development of cardiac hypertrophy and fibrosiss ${ }^{2-4}$. Manipulation of the immune response may prevent progression of heart failure. For instance, adoptive transfer of immunosuppressive T-regulatory cells ${ }^{5}$ and polarization of macrophages towards a more anti-inflammatory phenotype attenuate cardiac hypertrophy and dysfunction ${ }^{6,7}$.

CD40 (or TNFRSF5) is a cell-surface receptor belonging to the tumor necrosis factor (TNF) receptor super family and is predominantly expressed by antigen-presenting cells. Its corresponding ligand (CD40L or CD154) is a receptor expressed on T-cells and platelets and can be cleaved into a soluble form (sCD40L). The co-stimulatory dyad CD40L-CD40 is a critical regulator of the immune response in different inflammatory diseases like atherosclerosis, multiple sclerosis and arthritis ${ }^{8-10}$. Blocking of CD40L-CD40 interactions reduced disease severity. Importantly, serum levels of SCD40L are elevated in patients with HHD and HF ${ }^{11-13}$.

CD40L-CD40 interactions have pleiotropic effects depending on the cell-type and the downstream CD40-signaling pathways involved ${ }^{14}$. The CD40 receptor itself lacks intrinsic signalling activity. Upon activation, it recruits adaptor molecules (TNF Receptor-Associated Factors, or TRAFs) to its cytoplasmic domain to initiate downstream signalling. Ligation of CD40L to CD40 results in trimerization of CD40 and subsequent association of TRAF molecules to its TRAF2/3/5 and/or TRAF6 binding sites, ensuing in nuclear factor $K B$ (NFKB) signalling ${ }^{15}$. Downstream signalling via TRAF6 only activates the canonical NFKB pathway, whilst downstream signalling via TRAF $2 / 3 / 5$ signalling activates both the canonical and noncanonical pathway ${ }^{16}$. In atherosclerosis, disease progression is mediated by CD40-expressing hematopoietic cells and CD40-TRAF6 interactions in macrophages and dendritic cells are specifically required for the pro-atherogenic activity exerted by these cells ${ }^{17}$.

The possible pathophysiological significance of CD40L-CD40-TRAF signalling in the development of HHD has never been addressed. Accordingly, the present study reveals that absence of CD40 in KO mice and deficiency of CD40-TRAF binding in the myeloid cells results in a significant reduction of cardiac hypertrophy. 


\section{Material \& Methods}

\section{Mouse model of HHD}

Three months old male CD40 knockout (CD40 $\%, n=22)$, Wt $(n=23)$ and transgenic CD40-Twt $(n=16), C D 40-T \Delta 2(n=14) C D 40-T \Delta 6(n=13)$ and CD40-T $2 / 6(n=14)$ mice weighing $20-30$ gram were used (all on a C57Bl6 background ${ }^{18}$ ). Transgenic mice were on a $\mathrm{CD} 40^{\%}$ background expressing a chimeric CD40 transgene without (CD40-Twt) or with mutations either at the TRAF2/3/5 (CD40-T $\Delta 2$ ), TRAF6 (CD40-T $\Delta 6$ ) or both binding sites (CD40-T $\Delta 2 / 6)$. Expression of this transgene is under the control of the major histocompatibility complex class II (MHC class II) promotor ${ }^{18}$. To study the effect of HHD, AngII (Bachem AG, Bubendorf, Switzerland) was dissolved in sterile PBS and was infused subcutaneously ( $2.5 \mathrm{mg} / \mathrm{kg} / \mathrm{day})$ by osmotic mini pumps (ALZET, model 2004, DURECT corporation, Cupertino, USA) for 28 days. During mini pump implantation mice were anesthetized with isoflurane ( $2.5 \mathrm{Vol} \%$ isoflurane / air). In each Angll -treatment group 1-3 (6 to 21\%) mice died prematurely due to peri-aortic rupture and bleeding. All knockout and transgenic mice were bred and maintained in the animal facility of Maastricht University. The animals had free access to standard chow (SNIFF, Soest, Germany) and drinking water and were housed in groups of 2-5 mice per cage in a temperature-controlled room with $12 \mathrm{~h}: 12 \mathrm{~h}$ light-dark cycle. This study was approved by the institutional animal research ethics committee and was designed conform the guidelines for the use of laboratory animals formulated in the Dutch law on care and use of experimental animals.

\section{Echocardiographic Assessment of Cardiac Hypertrophy and Blood Pressure Measurement}

Cardiac dimensions and function were determined using echocardiography. Mice were anesthetized with isoflurane $(2.5 \mathrm{Vol} \%$ isoflurane / air) and analysed with a $30-\mathrm{MHz}$ transducer (RMV 707b, VisualSonics, Toronto, Canada). Two-dimensionally targeted B-mode echocardiographic images were recorded at the level of the papillary muscles from the shortaxis view. Intraventricular septal (IVS), left ventricular posterior wall thickness (LVPW) and left ventricular internal diameter (LVID) at systole and end-diastole were estimated from these images. From these parameters, fractional shortening (FS) was determined as the percentage decrease of LVID between diastole to systole. Wall thickness was expressed as the mean of IVS and LVPW thickness. Relative wall thickness is determined by dividing wall thickness by the LVID during diastole. Blood pressure was monitored using a tail cuff (CODA, Kent Scientific, Torrington, CT) in awake animals 3 days before sacrifice.

\section{In vitro experiments}

Isolation and activation of neonatal ventricular cardiomyocytes.

Rat neonatal ventricular cardiomyocytes (NCMs) were isolated by enzymatic dissociation from 1- to 2-day old neonatal rats. NCMs were plated $2.5 \times 10^{5}$ on gelatinized 6 -well plates and cultured as described previously ${ }^{19}$. NCMs were primed with IFNy 100u (Peprotech, London, UK) for $24 \mathrm{~h}$ and subsequently activated with an agonistic CD40 antibody, $10 \mathrm{\mu g} / \mathrm{ml}$ (FGK45, Bioceros, Utrecht, The Netherlands) or a Rat IgG isotype control (Santa Cruz Biotechnology, Heidelberg, Germany, Cat. No. SC-2026) for 6 hours to determine inflammatory markers and 24 hours to determine hypertrophic response with 3 replicates per condition. 
BMDM Conditioned medium experiment.

Bone marrow derived macrophages (BMDM) were generated as described before ${ }^{20}$. In brief, bone marrow was isolated from 12 weeks old $\mathrm{CD}_{4} \%$ and $\mathrm{Wt}$ mice and cultured in RPMI supplemented with $15 \%$ M-CSF containing 1929 cell conditioned medium (LCM) to generate BMDMs and lifted for plating at day 8. BMDM conditioned medium was collected after $24 \mathrm{~h}$ of LPS stimulation and was dialyzed for $24 \mathrm{~h}$ using a $40 \mathrm{~nm}$ membrane against a $10 \mathrm{x}$ higher volume of NCM medium. Dialyzed conditioned medium was subjected to NCMs in presence and absence of $10 \mathrm{nM}$ Endothelin-1 (ET-1) (Sigma-Aldrich, Zwijndrecht, The Netherlands, Cat No. E7764) with three replicates per condition. The experiment was repeated three times.

\section{Tissue preparation, histology and immunohistochemistry}

After 28 days of Angll infusion and immediately after echocardiography mice were sacrificed by exsanguinations via the abdominal aorta. Blood was collected in heparinized tubes and centrifuged for $15 \mathrm{~min}$ at $+4 \mathrm{C}$, obtained plasma was stored at $-80 \mathrm{C}$ until analysis. The ventricles, lung, liver, spleen and kidney were dissected, weighed and prepared for further analysis. Atria were removed from the heart and the ventricles were cut into two pieces between base and apex. Transverse midsections of the hearts were fixed in 1\% PFA for $4 \mathrm{~h}$ and embedded in paraffin. The tissue was sectioned in $5 \mu \mathrm{m}$ thick slices and later processed for Hematoxylin/Eosin, Picrosirius red and Wheat Germ Agglutinin (WGA) staining. The apex of the heart was sectioned into two parts and snap frozen for RNA and protein isolation.

Fibrosis was quantified as Sirius red positive area and peri-vascular fibrosis was excluded from the quantification. WGA staining was performed to determine cardiomyocyte cross sectional area by incubating deparaffinized and rehydrated slides for $30 \mathrm{~min}$ with $50 \mu \mathrm{g} / \mathrm{ml}$ FITC labelled Lectin (Sigma-Aldrich, Zwijndrecht, The Netherlands, Cat. No. L4895) and mounting with Vectashield + DAPI (Vector Laboratories, Peterborough, UK, Cat. No. H-1500). All quantifications were performed in a blinded manner using Leica Qwin software.

\section{Immunohistochemistry}

Paraffin sections were de-paraffinized and rehydrated. After boiling in a pressure cooker in $10 \mathrm{mM}$ sodium citrate buffer to unmask antigens, the slides were blocked in buffer containing normal Rabbit serum (Dako, Heverlee, Belgium, Cat. No. X0902,), incubated with primary antibody Rat-anti-Mouse CD45 (BD Pharmingen, Germany, Cat. No. 550539), and then incubated with biotin-conjugated secondary Rabbit-anti-Rat antibody (Dako, Heverlee, Belgium, Cat. No. E0468). Signal was amplified using TSA kit (PerkinElmer, USA, Cat. No. NEL747A) and visualized using DAB (Sigma-Aldrich, Zwijndrecht, The Netherlands, Cat. No. D5905). Three random photographs were taken at 20X magnification using a LEICA DM2000 microscope and LAS v3.8.0 software. CD45 positive cells per field were counted using ImageJ software.

\section{Analysis of cytokine levels in serum and BMDM conditioned medium}

Conditioned medium of unstimulated and LPS $(100 \mathrm{ng} / \mathrm{ml})$ stimulated CD40 $/-$ and Wt BMDM was analysed for pro-inflammatory cytokines IFN- $\gamma$, IL-10, IL-12p70, IL-13, IL-2, IL-4, IL-5, IL-6, KC/GRO and TNF- $\alpha$ using V-PLEX Pro-inflammatory Panel 1 (mouse) Kit (MSD, Cat. No. K15048D-1) according to manufacturer's protocol. 


\section{Gene expression analysis}

Left ventricular tissue was snap frozen in liquid nitrogen and processed for analysis of mRNA content by quantitative PCR. Total RNA was isolated using a RNeasy kit (Qiagen, Venlo, the Netherlands) following manufacturer's protocol. cDNA was prepared using the iScript kit (BioRad Inc., Hercules, CA, USA) using $1 \mu \mathrm{g}$ RNA of each sample as input. RT-qPCR and SYBR Green (BioRad Inc. Hercules, CA, USA) quantitative RT-PCR analysis was performed on a BioRad iCycler to determine the mRNA levels of the analysed genes. The $\Delta \Delta \mathrm{Ct}$ method was used to analyse obtained $\mathrm{Ct}$ Values and make the mRNA levels relative to the control groups and corrected for housekeeping gene GAPDH.

\section{Statistical Analysis}

Values are presented as MEAN \pm SEM. Data from different groups were analysed using twotailed Student's t testing for Gaussian data when two groups were compared. For comparisons of more than 2 groups, ANOVA in combination with Bonferroni or Dunnet's post testing was applied. For echo-analysis paired comparisons were used between baseline groups and 4 week Angll treated mice. Differences were considered statistically significant at $P$ values $<0.05$. 


\section{Results}

\section{Absence of CD40 blunts the hypertrophic response after Angll infusion}

To assess the role of CD40 in the development of cardiac hypertrophy, CD40 knockout $\left(\mathrm{CD} 40^{-/}\right)$and Wild type (Wt) mice were infused with Angll for 4 weeks. Arterial blood pressure rose to a similar extent in both $\mathrm{Wt}$ and $\mathrm{CD} 40^{-/}$mice in response to Angll (Supplemental Figure 1). Notably, ventricular mass increased significantly in $\mathrm{Wt}(+31 \%$, $\mathrm{P}<0.01)$, but not in $\mathrm{CD} 40^{\%}$ mice $(+10 \%, \mathrm{~N} . \mathrm{S}$.) in response to Angll treatment (Figure $1 \mathrm{a}$ and Table 1). In line, the echocardiographic diastolic LV wall thickness and histological cardiomyocyte cross sectional area (CSA) increased in $\mathrm{Wt}$, but not in CD40 ${ }^{-1-}$ mice (Figure $1 \mathrm{~b}$ c, Table 2). Also transcript levels of the cardiac hypertrophic markers $\alpha$-skeletal actin ( $\alpha$-SKA) and atrial natriuretic factor (ANF) remained low in Angll-treated CD40 $\%$ mice, as compared to Wt mice (Figure 1d-e, Supplemental table 1). Fractional shortening (FS) was reduced to a similar extent in Wt and $\mathrm{CD} 40^{-/}$mice following Angll-treatment (Figure 1f, Table 2). Also Sirius red stained interstitial fibrosis or transcript levels of Col1 $\alpha$ and TGF $\beta 1$ (both strongly increasing upon Angll infusion) did not differ significantly between Wt and $\mathrm{CD} 40^{-\%}$ hearts (Figure 2a, Supplemental table 1). Collectively, these findings indicate a blunted hypertrophic response in the absence of CD40. This blunted hypertrophy however did not affect cardiac fibrosis or overall function.

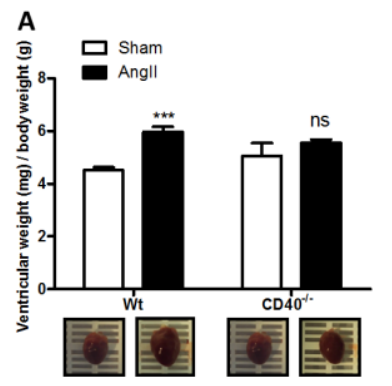

B

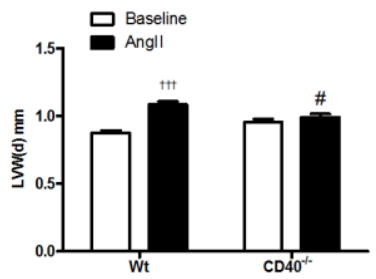

E

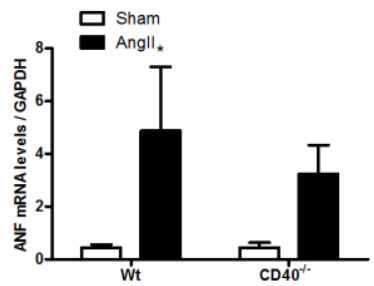

C
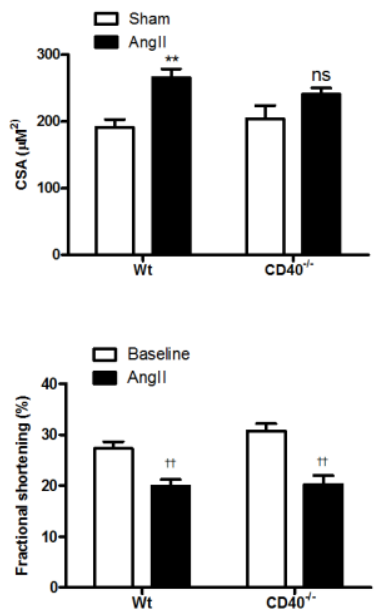

Figure 1. CD40\% mice have a blunted hypertrophic response after Angll treatment. CD40\% and Wt were infused with Angll (2.5 mg/kg/day) for 4 weeks. Untreated $\mathrm{CD}^{-1} \mathrm{O}^{-1}$ and Wt mice served as controls. A. Ventricular weight corrected for by body weight at the start of the experiment shows a significant increase in Wt mice after Angll but not in $\mathrm{CD}^{-1} \%$ mice. B. Echocardiographic measurements before (white bars) and after 4 weeks (black bars) of Angll treatment showed a strong increase in diastolic wall thickness in Angll treated Wt mice but no significant increase in Angll treated $\mathrm{CD}_{40} 0^{-}$mice. Wall thickness was shown as the average of intra-ventricular septal (IVS) and left ventricular posterior wall thickness (LVPW) in mm during diastole. C. Cardiomyocyte CSA shows a significant increase in Wt but not in CD40--mice upon Angll. D. Transcript levels of $\alpha$-SKA and ANF (panel E.) show a significant increase in Wt but not in $\mathrm{CD}^{-} 0^{-}$mice upon Angll. F. Fractional shortening, determined as a percentage decrease of LVID between diastole to systole, is significantly decreased in both Wt and $\mathrm{CD} 40^{\%}$ mice upon Angll infusion. Error bars represent SEM; * Compared to corresponding sham groups, \# Compared to Wt-Angll,

+ Compared to Baseline values. ${ }^{*} P<0.05 * * P<0.01{ }^{* * *} P<0.001$. 
Table 1. Morphometric characteristics of Angll-treated and non-treated Wt and CD40 $\%$ mice.

\begin{tabular}{lcccc}
\hline & Wt (N=11) & Wt Angll (N=16) & CD40\% (N=6) & CD40-/- AngII (N=22) \\
\hline Body Weight Day 0 (g) & $25.4 \pm 0.5$ & $26.3 \pm 0.6$ & $23.5 \pm 0.6$ & $24.6 \pm 0.4$ \\
Body Weight Day 28 (g) & $27.2 \pm 0.7$ & $27.6 \pm 0.6$ & $24.8 \pm 0.7$ & $26.0 \pm 0.4$ \\
Lung (mg) & $180 \pm 7$ & $215 \pm 7$ & $168 \pm 7$ & $197 \pm 11$ \\
Liver (mg) & $1064 \pm 98$ & $1062 \pm 34$ & $1188 \pm 41$ & $1015 \pm 43$ \\
Kidney (mg) & $169 \pm 4$ & $158 \pm 4$ & $169 \pm 8$ & $159 \pm 6$ \\
Spleen (mg) & $89.0 \pm 7$ & $86.9 \pm 4$ & $73.3 \pm 6$ & $78.4 \pm 3$ \\
Ventricles (mg) & $115 \pm 4$ & $155 \pm 6^{* * *}$ & $119 \pm 13$ & $137 \pm 4$
\end{tabular}

Data are expressed as means \pm S.E.M. For statistical analysis one-way ANOVA test with Bonferroni post-hoc testing was applied. ${ }^{*}$ indicates statistically significant difference $\left({ }^{*} P<0.05,{ }^{*} P<0.01,{ }^{* * *} P<0.001\right)$ between Anglltreated and corresponding non-treated mice.
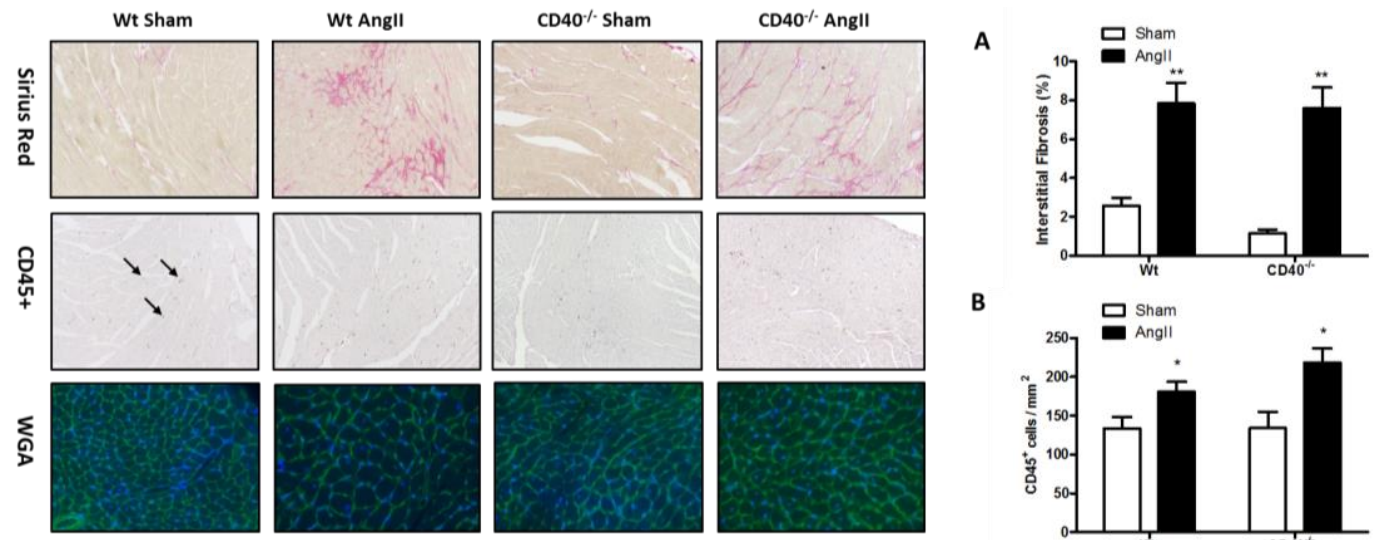

B

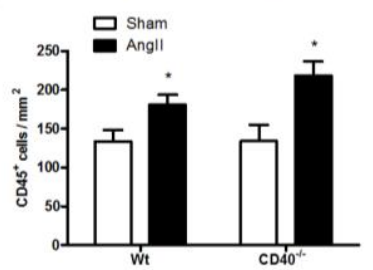

Figure 2. Histological analysis of myocardial tissue after Angll treatment. Panels with Sirius Red, CD45 immunostaining and WGA staining showing the effect of Angll treatment on the myocardium in Wt and CD40 ${ }^{-1}$ mice. A. Interstitial fibrosis is quantified as Sirius Red positive area and shows no difference between Wt and CD40/- mice. B. Loss of CD40 does not attenuate number of $\mathrm{CD}_{4} 5^{+}$leukocytes after Angll treatment. Number of leukocytes were determined as $C D 45^{+}$cells using IHC staining. The arrows indicate examples of $C D 45^{+}$cells. Error bars represent SEM; ${ }^{*} P<0.05 * * P<0.01 * * * P<0.001,{ }^{*}$ Compared to corresponding Sham group. 
Table 2. Echocardiographic determination of changes in cardiac dimensions and cardiac performance characteristics of Angll-treated and non-treated Wt and CD40--- mice.

\begin{tabular}{|c|c|c|c|c|}
\hline & Wt & Wt Angll & $\mathrm{CD} 40^{-/}$ & CD40\% AngII \\
\hline \multicolumn{5}{|c|}{ Heart rate (bpm) } \\
\hline - 0 days & $505 \pm 32$ & $502 \pm 16$ & $527 \pm 14$ & $513 \pm 16$ \\
\hline-28 days & $485 \pm 23$ & $498 \pm 21$ & $463 \pm 17$ & $530 \pm 15$ \\
\hline \multicolumn{5}{|c|}{ Wall thickness, diastolic (mm) } \\
\hline - O days & $0.86 \pm 0.02$ & $0.87 \pm 0.02$ & $0.86 \pm 0.01$ & $0.95 \pm 0.03$ \\
\hline - 28 days & $0.92 \pm 0.04$ & $1.08 \pm 0.02 * * *$ & $0.81 \pm 0.02$ & $0.99 \pm 0.02 * * * \#$ \\
\hline \multicolumn{5}{|c|}{ Wall thickness, systolic (mm) } \\
\hline - O days & $1.17 \pm 0.03$ & $1.19 \pm 0.03$ & $1.17 \pm 0.04$ & $1.25 \pm 0.03$ \\
\hline - 28 days & $1.20 \pm 0.05$ & $1.30 \pm 0.02$ & $1.14 \pm 0.05$ & $1.20 \pm 0.04$ \\
\hline \multicolumn{5}{|c|}{ Left Ventricular Internal Diameter ( $\mathrm{mm}$ ) Diastolic } \\
\hline - 0 days & $3.67 \pm 0.09$ & $3.77 \pm 0.05$ & $3.99 \pm 0.07$ & $3.72 \pm 0.6$ \\
\hline - 28 days & $3.78 \pm 0.08$ & $3.76 \pm 0.11$ & $4.24 \pm 0.11$ & $3.89 \pm 0.09$ \\
\hline \multicolumn{5}{|c|}{ Left Ventricular Internal Diameter ( $\mathrm{mm}$ ) Systolic } \\
\hline - 0 days & $2.67 \pm 0.07$ & $2.75 \pm 0.07$ & $2.89 \pm 0.05$ & $2.58 \pm 0.07$ \\
\hline - 28 days & $2.87 \pm 0.11$ & $3.01 \pm 0.11$ & $3.23 \pm 0.14$ & $3.12 \pm 0.12$ \\
\hline \multicolumn{5}{|c|}{ Rel. Wall Thickness (Diastolic) } \\
\hline - O days & $0.23 \pm 0.01$ & $0.23 \pm 0.01$ & $0.21 \pm 0.01$ & $0.25 \pm 0.01 *$ \\
\hline-28 days & $0.26 \pm 0.01$ & $0.31 \pm 0.01$ & $0.19 \pm 0.01$ & $0.25 \pm 0.01^{\#}$ \\
\hline \multicolumn{5}{|c|}{ Fractional Shortening (\%) } \\
\hline - O days & $27.1 \pm 0.99$ & $27.3 \pm 1.13$ & $27.7 \pm 0.72$ & $30.1 \pm 1.44$ \\
\hline - 28 days & $24.0 \pm 1.96$ & $19.9 \pm 1.29$ & $24.1 \pm 1.49$ & $20.2 \pm 1.78$ \\
\hline
\end{tabular}

Data are expressed as means \pm S.E.M. For statistical analysis one-way ANOVA test with Bonferroni post-hoc testing was applied. * indicates statistically significant difference $\left({ }^{*} P<0.05,{ }^{*} P<0.01, * * * P<0.001\right)$ between Anglltreated and corresponding non-treated mice. \# indicates statistically significant difference (\# $P<0.05, \# \# P<0.01$, \#\#\# $P<0.001)$ compared to corresponding Wt mice. 

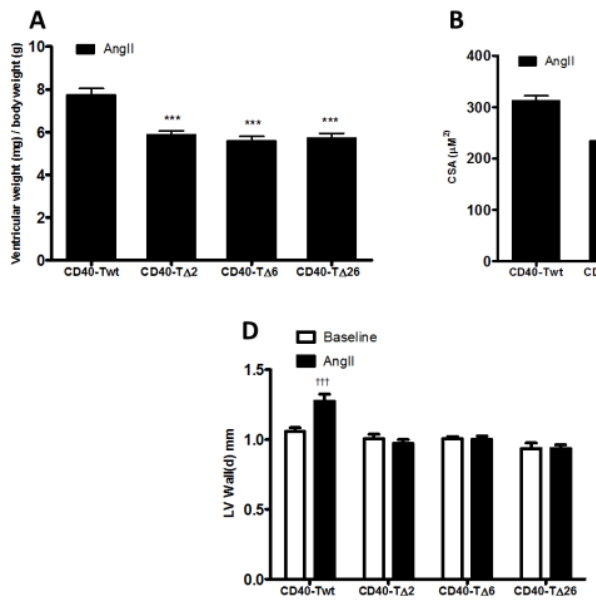

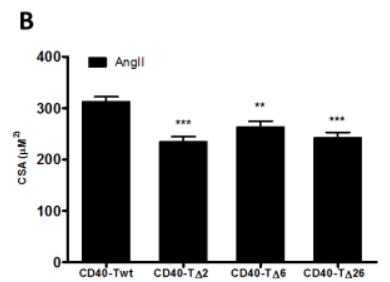

C

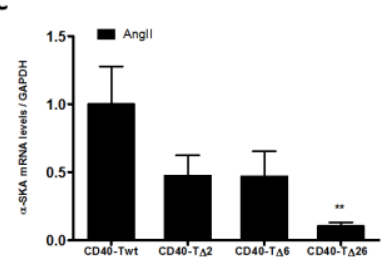

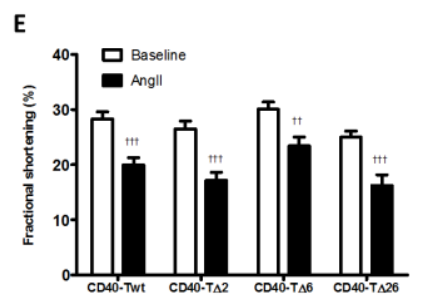

Figure 3. Loss of CD40-TRAF signalling reduces cardiac hypertrophy after Angll treatment. Transgenic CD40-Twt,

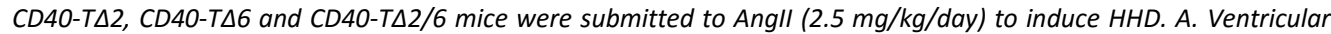

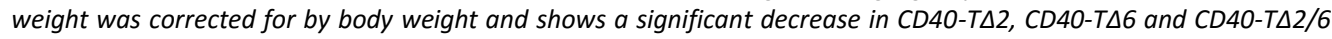
mice compared to CD4O-Twt controls. B. Cardiomyocytes of mice with disrupted CD4O-TRAF binding have reduced CSA after Angll treatment. C. Transcript levels of $\alpha$-SKA show a trend towards reduced expression in CD4O-TA2 and CD40-TA6 mice and was significantly decreased in CD40-TA2/6 mice. D. Echocardiographic measurements before (white bars) and after 4 weeks (black bars) of Angll treatment showed a strong increase in diastolic wall thickness in CD40-Twt mice but no increase in the groups with disrupted CD40-TRAF binding. Wall thickness was shown as mean of intra-ventricular septal (IVS) and left ventricular posterior wall thickness (LVPW) in mm during diastole. E. Fractional shortening was determined as a percentage decrease of LVID between diastole to systole. Disruption of CD4O-TRAF binding site does not improve fractional shortening after Angll treatment. Error bars represent SEM; * Compared to CD40-Twt Control, + Compared to corresponding baseline values. ${ }^{*} P<0.05 * * P<0.01 * * * P<0.001$.

\section{Both CD40-TRAF6 and CD40-TRAF2/3/5 interactions are implicated in cardiac hypertrophy}

To determine which of the CD40-TRAF signal transduction pathways is responsible for the blunted hypertrophic response in $\mathrm{CD}_{40}{ }^{-/}$mice, transgenic mice with disrupted CD40-TRAF binding were submitted to 4 weeks of Angll infusion. The mice are on a CD40\%- background and express a chimeric CD40 transgene, without (CD40-Twt) or with mutations either at the

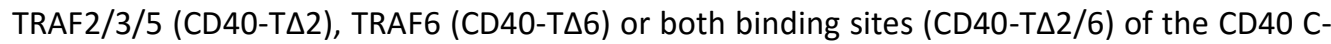
terminus. As transgene expression is under control of the major histocompatibility complex class II (MHC class II) promoter ${ }^{18}$, its expression is confined to antigen presenting cells (APCs), mostly macrophages, B-cells and dendritic cells. Baseline body weight, heart weight and serum levels of SCD40L did not significantly differ between the genotypes as was shown previously ${ }^{17,21}$.

Baseline echocardiographic measurements revealed only modest differences in cardiac function and dimensions (Table 3). Only in CD40-T $\Delta 2 / 6$ mice, baseline values of wall thickness (LVWd, -11\%) and left ventricular internal diameter (LVIDd, $+8.5 \%$ ) significantly changed compared to CD40-Twt control. Upon Angll infusion, ventricular mass was remarkably large (VW/BW Ratio $7.73 \pm 0.31$ ) in transgenic mice with intact CD40-TRAF signalling (CD40-Twt) and significantly larger than in Wt mice (VW/BW ratio $5.96 \pm 0.20$ ) (Figure $3 a$ and 1a). Disruption of TRAF binding sites resulted in significantly lower cardiac mass after Angll treatment vs. intact CD40-TRAF signalling (VW/BW Ratio -23.9\% in CD40$\mathrm{T} \Delta 2,-26.1 \%$ in CD40-T $\Delta 6$ and $-27.8 \%$ in CD40-T $\Delta 2 / 6, \mathrm{p}<0.001$ ) (Figure 3a, Table 4). Diastolic LV wall thickness, concordantly to the results above, increased significantly in CD40-Twt after 
Angll compared to baseline (Figure 3d). In the mutant groups however, the increase in LV wall thickness was completely blunted (Figure 3d, Table 3).

Table 3. Echocardiographic determination of changes in cardiac dimensions and cardiac performance in of transgenic mice with disrupted CD40-TRAF binding treated with angiotensin II (AngII) for 4 weeks.

\begin{tabular}{|c|c|c|c|c|}
\hline & CD40-Twt (N=16) & $\mathrm{CD} 40-\mathrm{T} \Delta 2(\mathrm{~N}=12)$ & $\mathrm{CD} 40-\mathrm{T} \Delta 6(\mathrm{~N}=14)$ & $\mathrm{CD} 40-\mathrm{T} \Delta 2 / 6(\mathrm{~N}=12)$ \\
\hline \multicolumn{5}{|c|}{ Heart rate (bpm) } \\
\hline - 0 days & $482 \pm 20$ & $516 \pm 17$ & $516 \pm 14$ & $529 \pm 14$ \\
\hline - 28 days & $557 \pm 11$ & $535 \pm 18$ & $525 \pm 20$ & $515 \pm 24$ \\
\hline \multicolumn{5}{|c|}{ Wall thickness, diastolic (mm) } \\
\hline - 0 days & $1,06 \pm 0.03$ & $1,01 \pm 0.03$ & $1,00 \pm 0.01$ & $0,94 \pm 0.04 * *$ \\
\hline - 28 days & $1,27 \pm 0.05$ & $0,96 \pm 0.03 * * *$ & $1,01 \pm 0.02 * * *$ & $0,95 \pm 0.02 * * *$ \\
\hline \multicolumn{5}{|c|}{ Wall thickness, systolic (mm) } \\
\hline - 0 days & $1.36 \pm 0.02$ & $1.29 \pm 0.04$ & $1.28 \pm 0.02$ & $1.12 \pm 0.02 * * *$ \\
\hline - 28 days & $1.49 \pm 0.06$ & $1.15 \pm 0.03 * * *$ & $1.32 \pm 0.04^{*}$ & $1.15 \pm 0.03 * * *$ \\
\hline \multicolumn{5}{|c|}{$\begin{array}{l}\text { Left Ventricular Internal } \\
\text { Diameter }(\mathrm{mm}) \text { Diastole }\end{array}$} \\
\hline - 0 days & $3.52 \pm 0.10$ & $3.52 \pm 0.07$ & $3.66 \pm 0.90$ & $3.82 \pm 0.06 *$ \\
\hline - 28 days & $4.12 \pm 0.14$ & $4.18 \pm 0.11$ & $3.93 \pm 0.12$ & $4.24 \pm 0.14$ \\
\hline \multicolumn{5}{|c|}{$\begin{array}{l}\text { Left Ventricular Internal } \\
\text { Diameter }(\mathrm{mm}) \text { Systole }\end{array}$} \\
\hline - 0 days & $2.51 \pm 0.07$ & $2.58 \pm 0.06$ & $2.57 \pm 0.9$ & $2.86 \pm 0.06 * *$ \\
\hline - 28 days & $3.31 \pm 0.15$ & $3.46 \pm 0.11$ & $3.02 \pm 0.15$ & $3.55 \pm 0.17$ \\
\hline \multicolumn{5}{|c|}{ Rel. Wall Thickness, diastolic } \\
\hline - 0 days & $0.31 \pm 0.01$ & $0.29 \pm 0.01$ & $0.28 \pm 0.01$ & $0.25 \pm 0.01 * *$ \\
\hline - 28 days & $0.31 \pm 0.02$ & $0.23 \pm 0.01^{* * *}$ & $0.26 \pm 0.01^{*}$ & $0.23 \pm 0.01^{* * *}$ \\
\hline \multicolumn{5}{|c|}{ Fractional Shortening (\%) } \\
\hline - 0 days & $28.3 \pm 1.3$ & $26.5 \pm 1.5$ & $30.1 \pm 1.3$ & $25.0 \pm 1.1$ \\
\hline - 28 days & $19.9 \pm 1.4$ & $17.2 \pm 1.5$ & $23.4 \pm 1.7$ & $16.2 \pm 1.9 *$ \\
\hline
\end{tabular}

Data are expressed as means \pm S.E.M. For statistical analysis one-way ANOVA test was used. With Dunnett's posthoc testing vs. CD40-Twt group. * indicates significant differences between CD40-Twt and the groups with disrupted TRAF binding. ( $P<0.05, * * P<0.01, * * * P<0.001)$.

Decreased hypertrophy in mice with disabled CD40-TRAF2/3/5 or/and CD40-TRAF6 binding was confirmed by a blunted increase in cardiomyocyte CSA (Figure $3 b$ ). Transcript levels of $\alpha$ SKA were significantly reduced in CD40-T $\Delta 26$ mice and tended to be reduced in CD40-T $\Delta 2$ and CD40-T $\Delta 6$ mice (Figure 3c, Supplemental table 2). Blood pressure and heart rate did not significantly differ among the 4 groups (Supplemental Figure 2). These findings show that both CD40-TRAF2/3/5 and CD40-TRAF6 signalling are involved in the development of cardiac hypertrophy. Despite attenuation of hypertrophy after Angll treatment, but in line with the result in $\mathrm{CD}_{40} \%$ mice, fractional shortening was not improved by disruption of CD40TRAF2/3/5 or CD40-TRAF6 interactions (Figure 3e, Table 3). Leukocyte numbers (CD45+ cells) 
did not differ significantly in the myocardium of these four groups of transgenic mice (Supplemental Figure 3). Also interstitial cardiac fibrosis and transcript levels of fibrotic markers did not differ significantly between CD40-T $\Delta 2, \mathrm{CD} 40-\mathrm{T} \Delta 6$, and CD40-T $\Delta 2 / 6$ and CD40-Twt mice and Wt mice (Supplemental Figure 3, Supplemental table 2).

Table 4. Morphometric characteristics of transgenic mice with disrupted CD40-TRAF binding all treated with Angll.

\begin{tabular}{|c|c|c|c|c|}
\hline & CD40-Twt (N=16) & CD40-T $\Delta 2(\mathrm{~N}=12)$ & CD40-T $\Delta 6(\mathrm{~N}=14)$ & $\mathrm{CD} 40-\mathrm{T} \Delta 2 / 6(\mathrm{~N}=12)$ \\
\hline Body Weight Start (g) & $25.6 \pm 0.7$ & $24.9 \pm 0.8$ & $25.0 \pm 0.7$ & $24.7 \pm 0.5$ \\
\hline Body Weight End (g) & $25.6 \pm 0.4$ & $24.9 \pm 0.4$ & $25.1 \pm 0.5$ & $25.5 \pm 0.4$ \\
\hline Lung (mg) & $195 \pm 9$ & $181 \pm 5$ & $203 \pm 8$ & $187 \pm 10$ \\
\hline Liver (mg) & $1178 \pm 57$ & $1083 \pm 51$ & $1130 \pm 32$ & $1122 \pm 49$ \\
\hline Kidney (mg) & $163 \pm 5$ & $168 \pm 7$ & $154 \pm 5$ & $165 \pm 5$ \\
\hline Spleen (mg) & $74 \pm 3$ & $76 \pm 3$ & $86 \pm 3^{*}$ & $88 \pm 3^{* *}$ \\
\hline Ventricles (mg) & $199 \pm 9$ & $151 \pm 5 * * *$ & $142 \pm 7 * * *$ & $146 \pm 7^{* * *}$ \\
\hline
\end{tabular}

Data are expressed as means \pm S.E.M. For statistical analysis one-way ANOVA test was used. With Dunnett's posthoc testing vs. CD40-Twt group. * indicates significant differences between CD40-Twt and the groups with disrupted TRAF binding. ( $\left.{ }^{*} P<0.05, * * P<0.01, * * * P<0.001\right)$.

\section{CD40 activation indirectly stimulates cardiomyocyte hypertrophy}

The number of leukocytes (CD45 $5^{+}$cells) in the myocardium increased equally in both Wt and $\mathrm{CD}^{\circ} \%$ mice after Angll (Figure 2b). However, transcript levels of M1 (iNOS) and M2 (Arginase-1) macrophage markers in the myocardium (Supplemental table 1), suggest a shift towards a more anti-inflammatory $\mathrm{M} 2$ phenotype in $\mathrm{CD} 40^{-1-}$ mice.

Besides immune cells, CD40 is also expressed by cardiomyocytes ${ }^{22}$. To determine whether activation of CD40 on cardiomyocytes can directly induce hypertrophy, rat NCMs were subjected to an agonistic CD40 (FGK45) antibody or an isotype control, and stimulated with Endothelin-1 (ET-1). Activation of CD40 on NCMs by the agonistic CD40 antibody FGK45 increased the transcript levels of pro-inflammatory genes, MCP-1, MIP- $1 \alpha$ and IL- 6 after $6 \mathrm{~h}$, indicative of successful CD40 ligation (Figure 4b). However, CD40 activation did not induce cardiomyocyte hypertrophy: neither transcript levels of ANP and $\alpha$-SKA (Figure 4b) nor cell surface area at $24 \mathrm{~h}$ increased significantly upon FGK45 treatment. To substantiate the notion that CD40 influences macrophage polarization, bone marrow derived macrophages (BMDMs) were generated from $\mathrm{Wt}$ and $\mathrm{CD} 40^{-/}$mice and activated with LPS. Conditioned medium of these BMDMs mice was administered to rat neonatal cardiomyocytes (NCMs) and a hypertrophic response was induced by stimulation with ET-1.

The conditioned medium from $\mathrm{CD}_{40} \%$ BMDMs blunted the $\mathrm{ET}-1$ induced hypertrophic response as shown by the reduction in both cell surface area and transcript levels of hypertrophic markers (Figure 4a). Subsequently we determined the levels of several cytokines in the conditioned medium. LPS stimulation induced pronounced increase of all cytokines measured. Although no significant difference in cytokine levels between Wt and CD40 $\%$ LPS treated BMDMs was observed, the pro-inflammatory cytokines IL-1 $\beta$, TNF $\alpha$, IL- 6 and IL-12 tended to be decreased in $\mathrm{CD}_{40}^{-/}$BMDM conditioned medium. Moreover, IL-6 levels were significantly lower in the conditioned medium of untreated $\mathrm{CD} 40^{-1-}$ BMDMs compared to Wt (Supplemental figure 4c). 

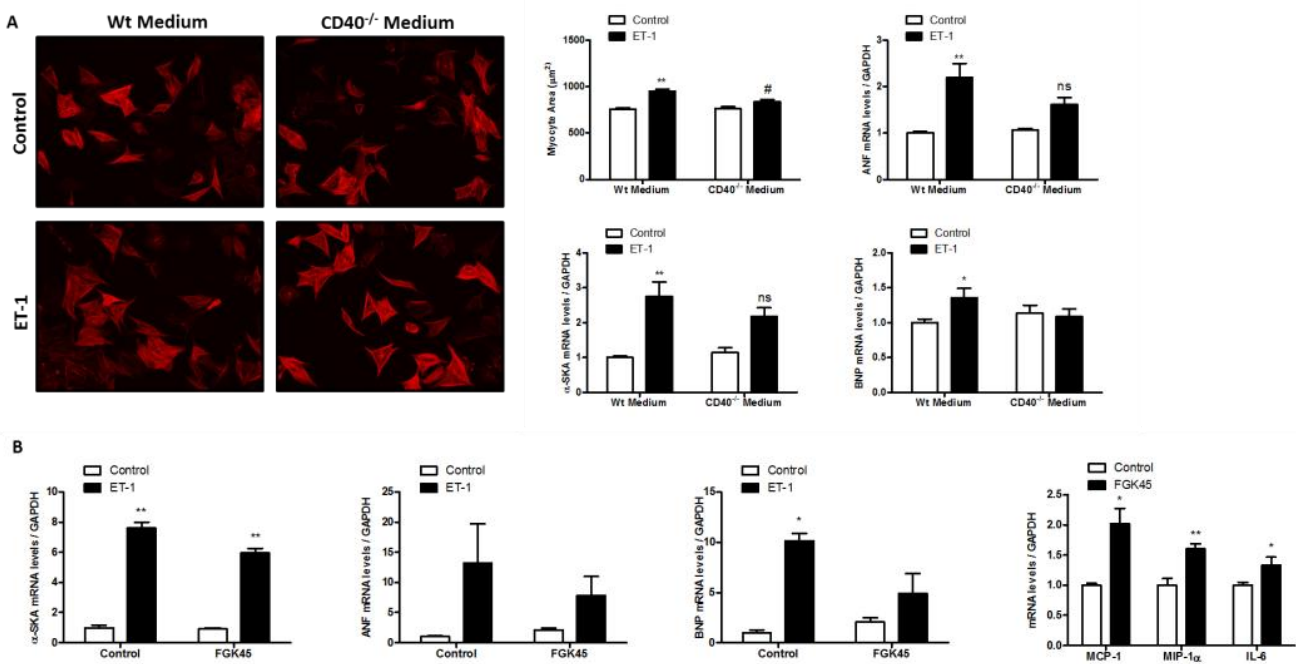

Figure 4. Conditioned medium of CD40\% BMDMs blunts hypertrophic response in NCMs. NCMs were cultured in conditioned medium of $C D 40^{-}$and Wt BMDMs and stimulated with ET-1. A. Phalloidin stained NCMs with $24 h$ ET-1 stimulus or control, quantification of cell surface area shows a reduction cell size for NCMs treated with conditioned medium of $\mathrm{CD}_{40} \%$ BMDMs compared to NCMs treated with conditioned medium of Wt BDMDs. The reduction in size is confirmed by transcript levels of hypertrophic markers $\alpha$-SKA, ANF and BNP. B. Activation of CD40 using an agonistic antibody (FGK45) does not induce hypertrophy on cardiomyocytes. FGK45 does not induce expression of marker genes $\alpha$-SKA, ANF and BNP alone nor does it affect expression in combination with ET-1 stimulation after $24 \mathrm{~h}$. To confirm activation of CD40 on cardiomyocytes by FGK45 transcript levels of marker genes, MCP-1, MIP-1 $\alpha$ and IL-6 were quantified after 6 h of activation. Error bars represent SEM; *Compared to corresponding Sham, \# Compared to Wt-Angll. ${ }^{*} P<0.05 * * P<0.01 * * * P<0.001$. 


\section{Discussion}

The present study reveals that the absence of the immuno-modulatory co-stimulatory receptor CD40 blunts cardiac hypertrophy upon pressure overload. Also expression of transgenic CD40 in MHC class $\mathrm{II}^{+}$cells exacerbates myocyte hypertrophy, while loss of CD40TRAF2/3/5 or/and CD40-TRAF6 interactions reduces hypertrophy. Furthermore, hypertrophy is inhibited when cardiomyocytes are exposed to conditioned medium from macrophages lacking CD40. Collectively, these results demonstrate that CD40L-CD40-TRAF interactions in antigen-presenting cells regulate Angll induced cardiac hypertrophy in a paracrine manner.

\section{CD40L-CD40 interactions control cardiac hypertrophy}

The current finding that $\mathrm{CD} 40$ is involved in cardiac hypertrophy is in line with previous studies that already suggested a potential role for CD40 the development of cardiac hypertrophy in patients, but did not provide biological evidence. CD40L expression on platelets and SCD40L levels in serum positively correlated with increased HF (NYHA) functional class ${ }^{23}$, left ventricular ejection fraction ${ }^{24}$ and hypertension ${ }^{11}$. Specific proinflammatory pathways, including TNF Receptor $1^{25}$ and TNF itself ${ }^{26}$, MCP-1 ${ }^{3,27}$ and IL-6 ${ }^{28}$ ${ }^{29}$ modulate cardiac hypertrophy upon pressure overload. Although activation of CD40 clearly stimulated cardiomyocyte hypertrophy, the Angll-induced fibrotic response or systolic dysfunction remained unaltered in both $\mathrm{CD} 40^{-}$mice and mice with mutated CD40-TRAF binding domains (figures $2 \mathrm{a}$ and supplemental 3 ). This suggests that fibrotic remodelling and associated systolic impairment is independent of CD4OL-CD40 signalling and contrasts with most other studies in which deletion of other inflammatory activators ${ }^{26,}{ }^{29}$ resulted in a blunted fibrotic response and decrease in contractility. Previously, however, some studies have also shown effects on hypertrophy independent of the fibrotic response ${ }^{7}$ or cardiac function ${ }^{30}$.

Notably, in mice with APC-specific expression of CD40 (CD40-Twt mice), Angll induced a far more pronounced hypertrophy (VW/BW ratio $7.73 \pm 0.31$ ) as compared to $\mathrm{Wt}$ mice (VW/BW ratio $5.96 \pm 0.20$, Figures $1 \mathrm{a}$ and $3 \mathrm{a}$ ). This underscores the importance of CD40 in cardiac hypertrophic remodelling. Moreover, as macrophages and dendritic cells are the two most common types of APCs expressing MHC class II in the hypertensive heart, this implies that expression of CD40 in these cell lineages is crucial for CD40-mediated cardiac hypertrophy. In addition to the increased SCD40L levels in the blood of hypertensive patients ${ }^{12}$, Angll has also been shown to directly induce the expression of CD40 on human monocytes and dendritic cells ${ }^{31}$. This could have an enhancing effect on the mechanism of myeloid CD40 mediated cardiac hypertrophy.

Both CD40-TRAF6 and CD40-TRAF2/3/5 interactions mediate cardiac hypertrophy after Angll induced pressure overload

Whereas in atherosclerosis, disease progression is mainly mediated by APC-specific CD40TRAF6 signalling ${ }^{17}$, our results here demonstrate that both CD40-TRAF6 and CD40TRAF2/3/5 interactions are involved in the development of cardiac hypertrophy (Figure 3). Animals that are deficient in either CD40-TRAF6 interactions (CD40-TA6 mice) or CD40TRAF2/3/5 interactions (CD40-T $\triangle 2$ mice) are protected from Angll-mediated cardiac hypertrophy. In animals with both CD40-TRAF6 and CD40-TRAF2/3/5 interactions blocked (CD40-TA2/6 mice) an expected additional reduction of hypertrophy was absent. This indicates that the pathway downstream of CD40-TRAF signalling involved in hypertrophy is 
activated via both TRAF6 and TRAF2/3/5. CD40 can activate NFKB, via the canonical and the non-canonical NFKB pathway. Interestingly, downstream CD40 signalling via TRAF6 activates the canonical NFKB pathway only, whereas CD40-TRAF2/5 interactions activate both pathways ${ }^{16}$. Classical NFKB activation has been shown to mediate cardiac hypertrophy in several mouse models of $\mathrm{HF}^{32-35}$ and leads to expression of pro-inflammatory and prohypertrophic cytokines such as IL-6. The blunted hypertrophic response in both CD40-T 22 and CD40-T $\triangle 6$ mice suggests involvement of the canonical pathway here.

\section{Polarization of macrophages lacking CD40L-CD40 signalling}

Interestingly, the amount of leukocytes $\left(\mathrm{CD}^{2} 5^{+}\right)$was comparable in the myocardium of $\mathrm{CD} 40^{-}$ 1- and Wt mice after Angll. However, we and others ${ }^{17,}{ }^{36}$, show that in vitro M1 polarization towards pro-inflammatory macrophages is abrogated and skews away from M1 polarization in the absence of CD40 signalling. Polarization towards M1 is known to increase cardiac hypertrophy and failure after pressure overload ${ }^{6,7}$. Data on $M 1$ versus $M 2$ marker transcript levels of macrophages in the myocardium are difficult to interpret because of bias from transcripts originating from other cardiac cell types such as cardiomyocytes and fibroblasts. We observed a shift away from the $\mathrm{M} 1$ marker in the myocardium of $\mathrm{CD} 40^{-/-}$mice, with lower transcript levels of iNOS and IL-12 in particular. Mice with mutated CD40-TRAF binding had lower cardiac transcript levels of M1 marker iNOS and higher levels of Arg-1. However, the fact that $\mathrm{CD} 40^{-/}$BMDM conditioned medium blunted the hypertrophic response in ET-1 treated cardiomyocytes (Figure 3) provides evidence for a change in the secretome of these BMDMs. Analysis of several cytokines in this conditioned medium showed only modest changes (Supplemental figure 4) indicating that the anti-hypertrophic effects of CD40\% BMDM conditioned medium are not mediated by these cytokines. Moreover, LPS stimulation induces a very pronounced increase in cytokine secretion, perhaps indicating that this trigger is too strong to detect subtler differences in cytokine synthesis between the two genotypes. The significantly lower IL-6 levels, a well-known pro-hypertrophic cytokine ${ }^{29}$, in unstimulated CD40\% compared to Wt BMDMs suggests that this might be the case.

\section{Conclusions}

We have shown that the absence of CD40L-CD40 signalling modulates cardiac hypertrophy. Furthermore, our results show that both downstream pathways via CD40-TRAF6 and CD40TRAF2/3/5 are mechanistically involved in in this process. Although cardiomyocyte hypertrophy during pressure overload is a highly complex process involving many cell types and signalling pathways, we provide additional evidence for the importance of myeloid cells in this process. The CD40L-CD40 co-stimulatory dyad offers opportunities for therapeutic intervention, both intra- and extra-cellular, to attenuate cardiac hypertrophy and thereby to treat hypertensive heart disease.

\section{Perspectives}

Pathological cardiac hypertrophy is a predictor of cardiac disease progression. CD40L has become an established biomarker of HF in hypertensive patients ${ }^{11,24}$. Our data provide biological evidence for a central role of CD40L-CD40-TRAF signalling plays in the development of cardiac hypertrophy. Blocking CD40L interactions has already been subject of clinical trials in various inflammatory diseases such as lupus and rheumatoid arthritis, but thromboembolic events have ended this ${ }^{37}$. The severe immune-compromising effects of blocking CD40 make long-term treatment difficult. Importantly, compounds specifically 
blocking CD40-TRAF6 interactions avoiding these side effects have been developed ${ }^{38}$ may have therapeutic potential of targeting the CD40L-CD40-TRAF pathway in the context of HHD.

\section{Acknowledgements}

Authors would like to thank Nicole Bitsch for excellent support with in vivo experiments and Rick van Leeuwen for isolation of neonatal cardiomyocytes. We acknowledge the support from the Netherlands Organization for Scientific Research (NWO) (VICl grant to E.L.), the Netherlands CardioVascular Research Initiative (CVON2011-19) and the Deutsche Forschungsgemeinschaft (DFG) (SFB1054-B04 to E.L. and SFB1123-A5 to E.L.). The research leading to these results has received funding from the European Union Commission's Seventh Framework program under grant agreement $N^{\circ} 602904$ (FIBROTARGETS, for fibrosis studies) and $N^{\circ} 261409$ (MEDIA, for macrophage/monocyte studies).

\section{References}

1. Heymans S, Hirsch E, Anker SD, Aukrust P, Balligand JL, Cohen-Tervaert JW, Drexler H, Filippatos G, Felix SB, Gullestad L, Hilfiker-Kleiner D, Janssens S, Latini R, Neubauer G, Paulus WJ, Pieske B, Ponikowski P, Schroen B, Schultheiss HP, Tschope C, Van Bilsen M, Zannad F, McMurray J and Shah AM. Inflammation as a therapeutic target in heart failure? A scientific statement from the Translational Research Committee of the Heart Failure Association of the European Society of Cardiology. Eur J Heart Fail. 2009;11:119-29.

2. Nicoletti A and Michel JB. Cardiac fibrosis and inflammation: interaction with hemodynamic and hormonal factors. Cardiovasc Res. 1999;41:532-43.

3. Kuwahara F, Kai H, Tokuda K, Takeya M, Takeshita A, Egashira K and Imaizumi T. Hypertensive myocardial fibrosis and diastolic dysfunction: another model of inflammation? Hypertension. 2004;43:739-45.

4. Hein S, Arnon E, Kostin S, Schonburg M, Elsasser A, Polyakova V, Bauer EP, Klovekorn WP and Schaper J. Progression from compensated hypertrophy to failure in the pressure-overloaded human heart: structural deterioration and compensatory mechanisms. Circulation. 2003;107:984-91.

5. Kvakan H, Kleinewietfeld M, Qadri F, Park JK, Fischer R, Schwarz I, Rahn HP, Plehm R, Wellner M, Elitok S, Gratze P, Dechend R, Luft FC and Muller DN. Regulatory T cells ameliorate angiotensin II-induced cardiac damage. Circulation. 2009;119:2904-12.

6. Usher MG, Duan SZ, Ivaschenko CY, Frieler RA, Berger S, Schutz G, Lumeng CN and Mortensen RM. Myeloid mineralocorticoid receptor controls macrophage polarization and cardiovascular hypertrophy and remodeling in mice. J Clin Invest. 2010;120:3350-64.

7. Heymans S, Corsten MF, Verhesen W, Carai P, van Leeuwen RE, Custers K, Peters T, Hazebroek M, Stoger L, Wijnands E, Janssen BJ, Creemers EE, Pinto YM, Grimm D, Schurmann N, Vigorito E, Thum T, Stassen F, Yin X, Mayr M, de Windt LJ, Lutgens E, Wouters K, de Winther MP, Zacchigna S, Giacca M, van Bilsen M, Papageorgiou AP and Schroen B. Macrophage microRNA-155 promotes cardiac hypertrophy and failure. Circulation. 2013;128:1420-32.

8. Lutgens E, Gorelik L, Daemen MJ, de Muinck ED, Grewal IS, Koteliansky VE and Flavell RA. Requirement for CD154 in the progression of atherosclerosis. Nat Med. 1999;5:1313-6.

9. Gerritse K, Laman JD, Noelle RJ, Aruffo A, Ledbetter JA, Boersma WJ and Claassen E. CD40-CD40 ligand interactions in experimental allergic encephalomyelitis and multiple sclerosis. Proceedings of the National Academy of Sciences of the United States of America. 1996;93:2499-504.

10. Durie FH, Fava RA, Foy TM, Aruffo A, Ledbetter JA and Noelle RJ. Prevention of collagen-induced arthritis with an antibody to gp39, the ligand for CD40. Science. 1993;261:1328-30.

11. Ueland T, Aukrust P, Yndestad A, Otterdal K, Froland SS, Dickstein K, Kjekshus J, Gullestad L and Damas JK. Soluble CD40 ligand in acute and chronic heart failure. Eur Heart J. 2005;26:1101-7.

12. Derosa G, D'Angelo A, Mugellini A, Pesce RM, Fogari E and Maffioli P. Evaluation of emerging biomarkers in cardiovascular risk stratification of hypertensive patients: a 2-year study. Curr Med Res Opin. 2012;28:1435-45. 
13. Damas JK, Otterdal K, Yndestad A, Aass H, Solum NO, Froland SS, Simonsen S, Aukrust P and Andreassen AK. Soluble CD40 ligand in pulmonary arterial hypertension: possible pathogenic role of the interaction between platelets and endothelial cells. Circulation. 2004;110:999-1005.

14. Engel D, Seijkens T, Poggi M, Sanati M, Thevissen L, Beckers L, Wijnands E, Lievens D and Lutgens E. The immunobiology of CD154-CD40-TRAF interactions in atherosclerosis. Semin Immunol. 2009;21:308-12.

15. Lutgens E, Lievens D, Beckers L, Donners M and Daemen M. CD40 and its ligand in atherosclerosis. Trends Cardiovasc Med. 2007;17:118-23.

16. Hauer J, Puschner S, Ramakrishnan P, Simon U, Bongers M, Federle C and Engelmann H. TNF receptor (TNFR)-associated factor (TRAF) 3 serves as an inhibitor of TRAF2/5-mediated activation of the noncanonical NF-kappaB pathway by TRAF-binding TNFRs. Proceedings of the National Academy of Sciences of the United States of America. 2005;102:2874-9.

17. Lutgens E, Lievens D, Beckers L, Wijnands E, Soehnlein O, Zernecke A, Seijkens T, Engel D, Cleutjens J, Keller AM, Naik SH, Boon L, Oufella HA, Mallat Z, Ahonen CL, Noelle RJ, de Winther MP, Daemen MJ, Biessen EA and Weber C. Deficient CD40-TRAF6 signaling in leukocytes prevents atherosclerosis by skewing the immune response toward an antiinflammatory profile. J Exp Med. 2010;207:391-404.

18. Ahonen C, Manning E, Erickson LD, O'Connor B, Lind EF, Pullen SS, Kehry MR and Noelle RJ. The CD40TRAF6 axis controls affinity maturation and the generation of long-lived plasma cells. Nat Immunol. 2002;3:451-6.

19. De Windt LJ, Willemsen PH, Popping S, Van der Vusse GJ, Reneman RS and Van Bilsen M. Cloning and cellular distribution of a group II phospholipase A2 expressed in the heart. J Mol Cell Cardiol. 1997;29:2095-106.

20. Peiser L, Gough PJ, Kodama T and Gordon S. Macrophage class A scavenger receptor-mediated phagocytosis of Escherichia coli: role of cell heterogeneity, microbial strain, and culture conditions in vitro. Infect Immun. 2000;68:1953-63.

21. Donners M, Beckers L, Lievens D, Munnix I, Heemskerk J, Janssen B, Wijnands E, Cleutjens J, Zernecke A and Weber $C$. The CD40-TRAF6 axis is the key regulator of the CD40/CD40L system in neointima formation and arterial remodeling. Blood. 2008;111:4596.

22. Seko Y, Takahashi N, Azuma M, Yagita H, Okumura K and Yazaki Y. Expression of costimulatory molecule CD40 in murine heart with acute myocarditis and reduction of inflammation by treatment with antiCD40L/B7-1 monoclonal antibodies. Circ Res. 1998;83:463-9.

23. Stumpf C, Lehner C, Eskafi S, Raaz D, Yilmaz A, Ropers S, Schmeisser A, Ludwig J, Daniel WG and Garlichs CD. Enhanced levels of CD154 (CD40 ligand) on platelets in patients with chronic heart failure. Eur J Heart Fail. 2003;5:629-37.

24. Yan JC, Liu PJ, Du RZ, Liang Y, Ma GS, Zhu J and Luo D. Relationship between CD40 ligand expression and B type natriuretic peptide levels in patients with chronic heart failure. Clin Chim Acta. 2008;392:17-20.

25. Duerrschmid C, Crawford JR, Reineke E, Taffet GE, Trial J, Entman ML and Haudek SB. TNF receptor 1 signalling is critically involved in mediating angiotensin-ll-induced cardiac fibrosis. J Mol Cell Cardiol. 2013;57:59-67.

26. Sun M, Chen M, Dawood F, Zurawska U, Li JY, Parker T, Kassiri Z, Kirshenbaum LA, Arnold M, Khokha R and Liu PP. Tumor necrosis factor-alpha mediates cardiac remodeling and ventricular dysfunction after pressure overload state. Circulation. 2007;115:1398-407.

27. Haudek SB, Cheng J, Du J, Wang Y, Hermosillo-Rodriguez J, Trial J, Taffet GE and Entman ML. Monocytic fibroblast precursors mediate fibrosis in angiotensin-ll-induced cardiac hypertrophy. J Mol Cell Cardiol. 2010;49:499-507.

28. Hirota H, Yoshida K, Kishimoto T and Taga T. Continuous activation of gp130, a signal-transducing receptor component for interleukin 6-related cytokines, causes myocardial hypertrophy in mice. Proc Natl Acad Sci U S A. 1995;92:4862-6.

29. Coles B, Fielding CA, Rose-John S, Scheller J, Jones SA and O'Donnell VB. Classic interleukin-6 receptor signaling and interleukin-6 trans-signaling differentially control angiotensin II-dependent hypertension, cardiac signal transducer and activator of transcription-3 activation, and vascular hypertrophy in vivo. The American journal of pathology. 2007;171:315-25.

30. Gao XM, Wong G, Wang B, Kiriazis H, Moore XL, Su YD, Dart A and Du XJ. Inhibition of mTOR reduces chronic pressure-overload cardiac hypertrophy and fibrosis. J Hypertens. 2006;24:1663-70.

31. Nahmod KA, Vermeulen ME, Raiden S, Salamone G, Gamberale R, Fernandez-Calotti P, Alvarez A, Nahmod V, Giordano $M$ and Geffner JR. Control of dendritic cell differentiation by angiotensin II. FASEB J. 2003;17:491-3.

32. Kawano S, Kubota T, Monden Y, Kawamura N, Tsutsui H, Takeshita A and Sunagawa K. Blockade of NFkappaB ameliorates myocardial hypertrophy in response to chronic infusion of angiotensin II. Cardiovasc Res. 2005;67:689-98. 
33. Freund C, Schmidt-Ullrich R, Baurand A, Dunger S, Schneider W, Loser P, El-Jamali A, Dietz R, Scheidereit C and Bergmann MW. Requirement of nuclear factor-kappaB in angiotensin II- and isoproterenol-induced cardiac hypertrophy in vivo. Circulation. 2005;111:2319-25.

34. Li Y, Ha T, Gao X, Kelley J, Williams DL, Browder IW, Kao RL and Li C. NF-kappaB activation is required for the development of cardiac hypertrophy in vivo. Am J Physiol Heart Circ Physiol. 2004;287:H1712-20.

35. Javan H, Szucsik AM, Li L, Schaaf CL, Salama ME and Selzman CH. Cardiomyocyte p65 nuclear factorkappaB is necessary for compensatory adaptation to pressure overload. Circulation Heart failure. 2015;8:109-18.

36. Verreck FA, de Boer T, Langenberg DM, van der Zanden L and Ottenhoff TH. Phenotypic and functional profiling of human proinflammatory type-1 and anti-inflammatory type-2 macrophages in response to microbial antigens and IFN-gamma- and CD40L-mediated costimulation. J Leukoc Biol. 2006;79:285-93.

37. Boumpas DT, Furie R, Manzi S, Illei GG, Wallace DJ, Balow JE, Vaishnaw A and Group BGLNT. A short course of BG9588 (anti-CD40 ligand antibody) improves serologic activity and decreases hematuria in patients with proliferative lupus glomerulonephritis. Arthritis Rheum. 2003;48:719-27.

38. van den Berg SM, Seijkens TT, Kusters PJ, Zarzycka B, Beckers L, den Toom M, Gijbels MJ, Chatzigeorgiou A, Weber C, de Winther MP, Chavakis T, Nicolaes GA and Lutgens E. Blocking CD40-TRAF6 interactions by small-molecule inhibitor 6860766 ameliorates the complications of diet-induced obesity in mice. International journal of obesity. 2014. 


\section{Supplementary Figures and Tables}

A

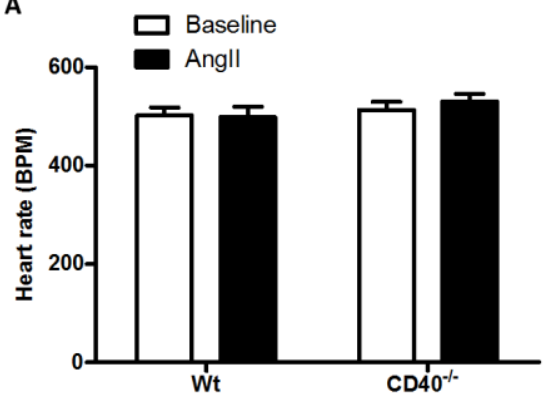

B

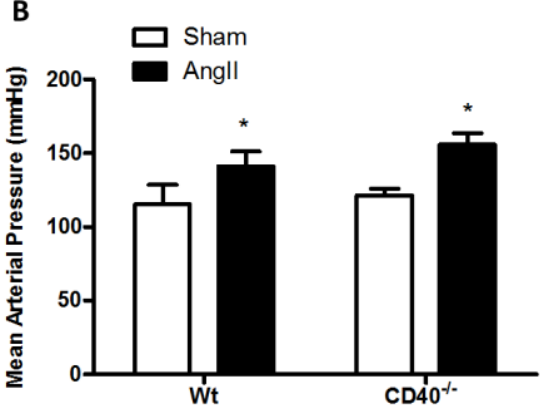

Supplemental figure 1. Heart rate and blood pressure of $\mathbf{W t}$ and $\mathrm{CD} 40 \%$ mice. Characteristics of Wt and CD40\% mice upon Angll infusion. A. Heart rate in beats per minute (BPM) does not differ between Wt and CD40\% mice or after Angll infusion. B. Mean arterial blood pressures were measured using tail cuff method after 26 days of Angll infusion, showing an increase in Wt and CD40\% mice after Angll treatment. Error bars represent SEM; *Compared to corresponding Sham.

A

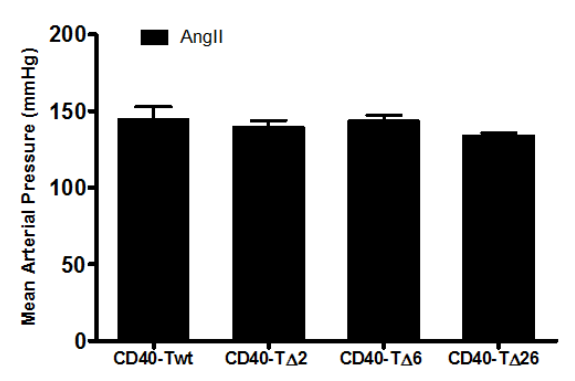

B

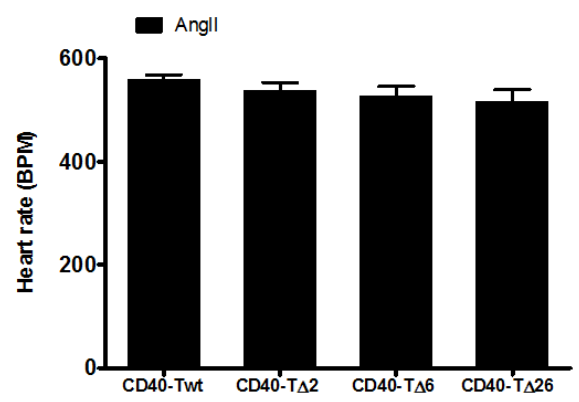

Supplemental figure 2. Heart rate and blood pressure of mice with disrupted TRAF binding. Characteristics of mice with disrupted TRAF binding upon Angll infusion. A. Mean arterial blood pressures were measured using tail cuff method after 26 days of Angll infusion, showing an equal level for all groups. B. Heart rate in beats per minute (BPM) does not differ between groups. Error bars represent SEM; ${ }^{*} P<0.05^{* *} P<0.01 * * * P<0.001$ to corresponding baseline group. 
A

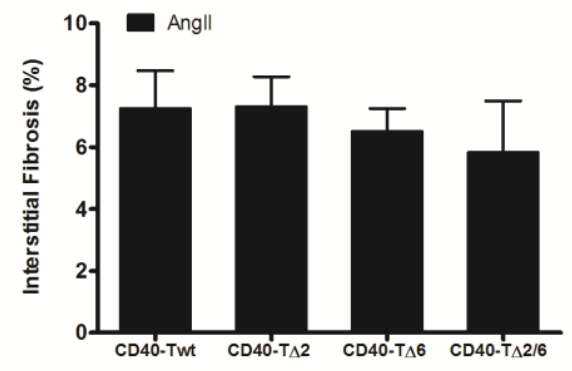

B

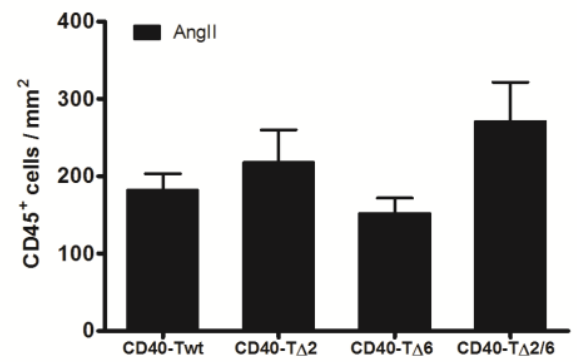

Supplemental figure 3. Histological analysis of myocardial tissue of mice with disrupted TRAF binding after Angll treatment. A. Fibrosis is unaffected in mice lacking CD40-TRAF signalling as determined by picrosirius red positive area. B. Loss of CD4O-TRAF signalling does not attenuate number of CD45+ leukocytes after Angll treatment. Number of leukocytes were determined as CD45 positive cells using IHC staining. Error bars represent SEM; * $P<0.05 * *$ $P<0.01 * * * P<0.001$, *Compared to corresponding Sham group

A

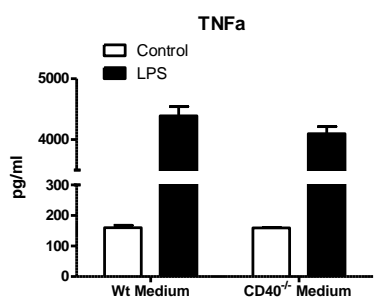

D

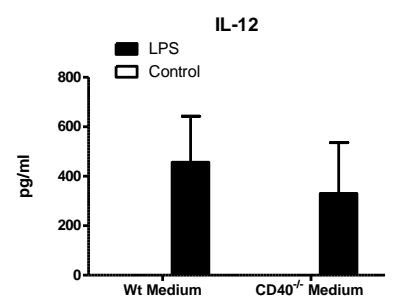

B

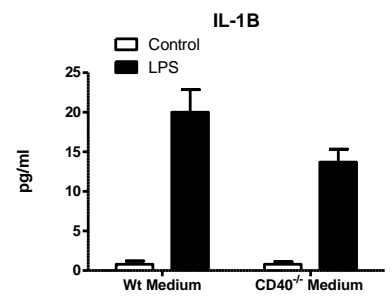

$\mathbf{E}$

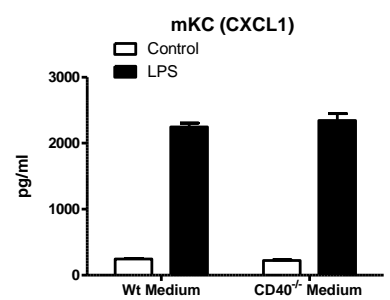

C

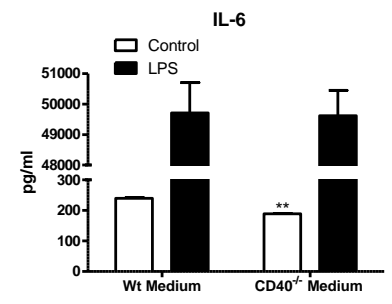

$\mathbf{F}$

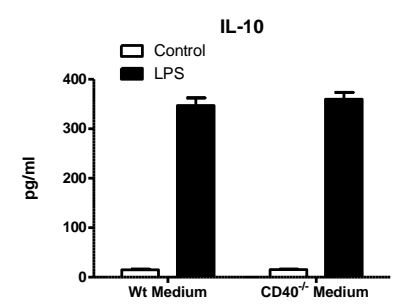

Supplemental figure 4. Cytokine levels in conditioned medium of Wt and CD40 $\%$ BMDM. Levels of TNF $, I L-16, I L-6$, $I L-12, K C$, and IL-10 (A-F) were determined in the supernatant of cultured BMDM using a V-PLEX Pro-inflammatory panel. LPS (100 $\mathrm{ng} / \mathrm{ml})$ induced expression of all measured cytokines. Only levels of IL-6 in control condition showed a significant difference between the genotypes. 
Supplemental table 1. Cardiac mRNA levels of marker genes for hypertrophy, fibrosis and inflammation of Wt and CD40 $\%$ mice treated with vehicle or angiotensin II (AngII) for 4 weeks.

\begin{tabular}{|c|c|c|c|c|c|}
\hline & $\mathrm{Ct}$ & Wt N=11 & Wt Angll N=16 & $\mathrm{CD} 40^{-/-\mathrm{N}=6}$ & CD40 $\%$ AngII N=22 \\
\hline \multicolumn{6}{|c|}{ Hypertrophy } \\
\hline$\alpha-S K A$ & 23.3 & $1.00 \pm 0.47$ & $2.80 \pm 0.61 *$ & $0.54 \pm 0.41$ & $1.62 \pm 0.27$ \\
\hline$A N F$ & 24.2 & $1.00 \pm 0.56$ & $4.87 \pm 2.44$ & $0.43 \pm 0.20$ & $3.23 \pm 1.10$ \\
\hline$B N P$ & 21.4 & $1.00 \pm 0.19$ & $1.91 \pm 0.33$ & $0.69 \pm 0.19$ & $1.74 \pm 0.25$ \\
\hline \multicolumn{6}{|c|}{ Fibrosis } \\
\hline Col1a & 27.1 & $1.00 \pm 0.18$ & $2.01 \pm 0.33$ & $0.59 \pm 0.06$ & $2.54 \pm 0.67$ \\
\hline$T G F b 1$ & 28.4 & $1.00 \pm 0.14$ & $1.40 \pm 0.18$ & $0.51 \pm 0.05$ & $1.76 \pm 0.29 *$ \\
\hline \multicolumn{6}{|c|}{ Inflammation / M1 Markers } \\
\hline iNOS & 31,1 & $1.00 \pm 0.55$ & $1.16 \pm 0.44$ & $0.59 \pm 0.26$ & $0.87 \pm 0.29$ \\
\hline IL-12 & 33.9 & $1.00 \pm 0.22$ & $1.07 \pm 0.21$ & $1.03 \pm 0.31$ & $0.71 \pm 0.11$ \\
\hline IL-6 & 33,2 & $1.00 \pm 0.46$ & $1.29 \pm 0.33$ & $0.30 \pm 0.10$ & $1.25 \pm 0.28$ \\
\hline$I L-16$ & 32.7 & $1.00 \pm 0.18$ & $0.81 \pm 0.10$ & $0.56 \pm 0.23$ & $0.85 \pm 0.18$ \\
\hline IKB $\alpha$ & 27.8 & $1.00 \pm 0.20$ & $1.01 \pm 0.09$ & $0.79 \pm 0.14$ & $1.31 \pm 0.16$ \\
\hline$M C P-1$ & 29.3 & $1.00 \pm 0.42$ & $0.47 \pm 0.01$ & $0.34 \pm 0.08$ & $0.51 \pm 0.05$ \\
\hline \multicolumn{6}{|c|}{ M2 Markers } \\
\hline $\operatorname{Arg}-1$ & 32,5 & $1.00 \pm 0.88$ & $0.22 \pm 0.11$ & $0.86 \pm 0.42$ & $0.13 \pm 0.06$ \\
\hline Fizz-1 & 31.7 & $1.00 \pm 0.20$ & $0.27 \pm 0.06 * *$ & $1.21 \pm 0.33$ & $0.32 \pm 0.08 * * *$ \\
\hline$Y M-1$ & 34.9 & $1.00 \pm 0.43$ & $1.30 \pm 0.35$ & $0.50 \pm 0.10$ & $1.04 \pm 0.18$ \\
\hline \multicolumn{6}{|c|}{ 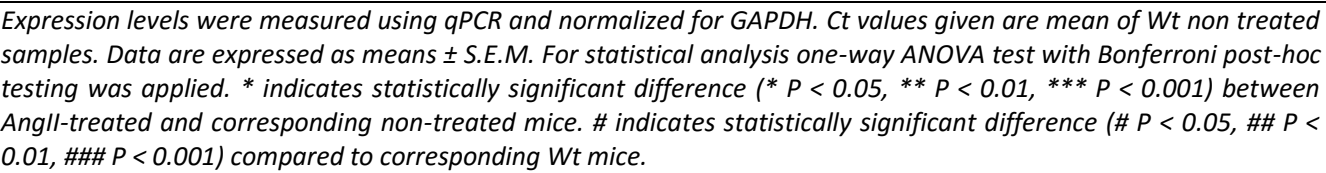 } \\
\hline
\end{tabular}


Supplemental table 2. Cardiac mRNA levels of marker genes for hypertrophy, fibrosis and inflammation of transgenic mice with disrupted CD40-TRAF binding treated Angll.

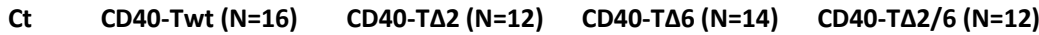

\section{Hypertrophy}

$\alpha-S K A$

ANF

BNP

Fibrosis

Col1a

TGFb1

Inflammation / M1

markers

iNOS

IL-6

MCP-1

$I L-16$

IL-12

$I K B \alpha$

\section{M2 Markers}

Arg-1

Fizz-1

$Y M-1$
19.4

$1.00 \pm 0.27$

21.4

$1.00 \pm 0.16$

19.7

$1.00 \pm 0.14$

23.7

$1.00 \pm 0.22$

$0.77 \pm 0.19$

$1.24 \pm 0.69$

$1.00 \pm 0.26$

$1.00 \pm 0.14$

$0.82 \pm 0.20$

$0.68 \pm 0.15$

$0.93 \pm 0.29$
31,9

$1.00 \pm 0.37$

$0.47 \pm 0.12$

$1.18 \pm 0.38$

$1.20 \pm 0.21$

$1.02 \pm 0.21$

$0.59 \pm 0.25$

$1.00 \pm 0.30$

25.3

33.7

$1.00 \pm 0.25$

34.88

$1.00 \pm 0.29$

$5.24 \pm 2.58$

$10.67 \pm 6.21$

$1.81 \pm 0.49$

$2.45 \pm 0.72$

$0.55 \pm 0.08$

$0.97 \pm 0.28$

33.6

$1.00 \pm 0.30$

$0.74 \pm 0.36$

$0.71 \pm 0.17$

$1.67 \pm 0.37$

$3.00 \pm 0.70 * *$

$1.02 \pm 0.16$

Expression levels were measured using qPCR and normalized for GAPDH. Ct values are mean CD40-Twt group. Data are expressed as means \pm S.E.M. For statistical analysis one-way ANOVA test was used. With Dunnett's post-hoc testing vs. CD40-Twt group. * indicates significant differences between CD40-Twt and the groups with disrupted TRAF binding. ( $P<0.05, * * P<0.01, * * * P<0.001)$. 


\section{Chapter 3}

\section{Inflammation-induced metabolic remodelling of the heart in acute viral myocarditis}

\section{Authors:}

Alex Remels ${ }^{1 *}$

Wouter Derks ${ }^{3 *}$

Koen Verhees ${ }^{1}$

Marco Kelders ${ }^{1}$

Paolo Carai ${ }^{3}$

Georg Summer ${ }^{3,4}$

Chiel de Theije ${ }^{1}$

Stephane Heymans ${ }^{3}$

Anna Papageorgiou ${ }^{3}$

Marc van Bilsen ${ }^{2,3}$

1.) Department of Respiratory Medicine, School for Nutrition, Toxicology \& Metabolism (NUTRIM), Maastricht University, Maastricht, the Netherlands

2.) Department of Physiology, Cardiovascular Research Institute Maastricht (CARIM), Maastricht University, Maastricht, the Netherlands

3.) Department of Cardiology, Cardiovascular Research Institute Maastricht (CARIM), Maastricht University, Maastricht, the Netherlands

4.) TNO, Microbiology \& Systems Biology, Zeist, the Netherlands

* AR and WD contributed equally

In preparation 


\section{Abstract}

Rationale: Abnormalities in cardiac energy metabolism, collectively referred to as cardiac metabolic remodelling, play an important role in the pathophysiology of heart failure. Although inflammation has been shown to interfere with regulatory pathways controlling cardiac metabolism, currently, it is unknown in which way, and to what extent, cardiac metabolism is perturbed in viral myocarditis (VM) and whether metabolic intervention has therapeutic potential in this condition.

Objectives: To delineate the effect of acute VM on cardiac metabolism in both humans and mice and to investigate potential molecular mechanisms underlying these metabolic disturbances. Additionally, we explored if dichloroacetate (DCA), an activator of glucose oxidation, has therapeutic potential in VM.

Methods and Results: Micro-array analysis of cardiac biopsies obtained from myocarditis patients revealed a marked down-regulation of several key pathways involved in oxidative metabolism, indicative of cardiac metabolic remodelling. Moreover, activity levels and protein/mRNA abundance of multiple constituents and molecular regulators of cardiac oxidative metabolism were decreased in Coxsackievirus B3 (CVB3)-induced VM in mice. Furthermore, cardiac NF-KB signalling was activated in both human and mouse VM and in vitro experiments using cultured cardiomyocytes revealed that NF-KB activation impairs cardiomyocyte oxidative metabolism by interfering with the peroxisome proliferatoractivated receptor $\gamma$ co-activator (PGC-1) network, the main regulatory pathway controlling cellular oxidative metabolism. Surprisingly, DCA treatment failed to restore VM-induced abnormalities in cardiac metabolic gene expression and, in fact, aggravated the VM-induced immune response and cardiac inflammation.

Conclusions: These findings collectively suggest that in acute VM the secretion of proinflammatory cytokines by infiltrating leukocytes activates NF-kB in cardiomyocytes, thereby interfering with the PGC1-mediated control of mitochondrial oxidative metabolism. DCAtreatment was unable to attenuate VM-induced disturbances in cardiac metabolic profile and function. 


\section{Introduction}

Characterized by an acute, fulminant inflammatory response in the heart, viral myocarditis (VM) is an important cause of death in young, otherwise healthy individuals. In a subgroup of patients VM gives rise to an autoimmune response predisposing to the development of dilated cardiomyopathy (DCM) ${ }^{1,2}$. Current treatment of VM focuses primarily on targeting the innate or acquired immune response ${ }^{3}$.

It is well established that cardiac disease is often accompanied by marked perturbations in cardiac energy metabolism, collectively referred to as cardiac metabolic remodelling ${ }^{4}$. Cardiac metabolic remodelling likely contributes significantly to disease progression 5, 6 and appears to be an attractive target for therapeutic intervention ${ }^{7}$. Stimulation of mitochondrial oxidative metabolism by dichloroacetate (DCA), for instance, was found to improve cardiac function and survival in a rat model of left ventricular hypertrophy and failure ${ }^{8}$. Although inflammation has been postulated as a potential driver of the metabolic remodelling process ${ }^{9}$, it is unknown whether disturbances in cardiac energy metabolism are involved in the development of VM-induced cardiac dysfunction.

Previously, it was shown that VM-induced cardiac dilatation upon exposure of mice to Coxsackievirus B3 (CVB3) was associated with reduced mRNA expression levels of genes involved in fatty acid $\beta$-oxidation and the mitochondrial respiratory chain ${ }^{10,11}$. Whether or not cardiac metabolism is perturbed in VM in humans is unexplored. Although these studies in mice suggest altered cardiac metabolic gene expression in VM, a comprehensive overview of the cardiac metabolic profile in VM is lacking. Furthermore, these studies do not allow one to discriminate whether these metabolic changes are due to (acute) viral myocarditis per se, or whether they arise secondary to the development of DCM over time. If impairments in cardiac oxidative energy metabolism are indeed involved in VM-induced cardiac dysfunction, this would imply that interventions aimed at improving cardiac metabolism may have a therapeutic benefit.

Previously, we and others showed that, both in the heart as well as in skeletal muscle tissue, activation of the inflammatory nuclear factor-KB (NF- $\mathrm{KB}$ ) signalling pathway induced a metabolic shift away from oxidative processes towards a greater reliance on, less efficient, glycolytic metabolism ${ }^{12,}{ }^{13}$. Therefore, we hypothesize that, in both humans and mice, the inflammatory response associated with acute VM ignites NF-KB-mediated reprogramming of cardiomyocyte energy metabolism thereby compromising cardiac energy status and leading to cardiac dysfunction. To address this, we first performed micro-array analysis on cardiac biopsies of patients with acute VM as well as on cardiac tissue of mice suffering from CVB3-induced VM and found clear evidence for a metabolic shift. Subsequently, we investigated the activity, as well as protein and mRNA levels of multiple constituents and molecular regulators of oxidative metabolism in intact hearts and in cardiac myocytes isolated from mice with CVB3-induced VM. Next, the impact of inflammation and activation of the NF-KB signalling pathway on metabolic gene expression in cultured cardiomyocytes was assessed in detail. Finally, we explored whether dichloroacetate (DCA), a drug known to stimulate mitochondrial oxidative metabolism, was able to ameliorate VMinduced cardiac injury. 


\section{Methods}

An expanded Material and Methods is available in the Supplemental material.

\section{Human subjects}

Acquisition of human samples and clinical parameters have been described previously ${ }^{14,15}$. In short, all human material was obtained during routine clinical sampling and available for research purposes in accordance with the Declaration of Helsinki and the ethical committee at Maastricht University. Written consent was obtained from all subjects. For micro-array analysis, right ventricular septal biopsies were used from patients with acute myocarditis $(n=3)$. Patients presented with an episode of upper airway or gastro-intestinal infection, followed after 1-3 weeks by chest pain. All myocarditis patients had elevated troponin T plasma levels and immunohistochemically proven cardiac inflammation ( $n>14$ CD45 positive cells $/ \mathrm{mm}^{2}$ ). In all patients, virus presence was demonstrated in cardiac biopsies ${ }^{14}, 15$. Coronary artery disease was excluded either by CAG or CT. Controls $(n=6)$ consisted of agematched patients with unexplained ventricular tachy-arrhythmias but with a normal ejection fraction and the absence of systemic or cardiac inflammation or virus presence at the time of biopsy ${ }^{14,15}$. Cardiac biopsies were immediately snap-frozen for analyses.

\section{Animal studies}

All mice were bred and maintained in an open animal facility (University of Leuven, Belgium) and experiments were performed according to the guidelines for the care and use of laboratory animals approved by the institution animal committee of the University of Leuven (ECD 2432013). 4-6 weeks old male wild type C3H mice (Harlan, NL) were injected intraperitoneally (i.p.) with CVB3 virus (Nancy strain, $200 \mu \mathrm{l} 5 \times 10^{8} \mathrm{PFU}$, derived from ATCC batch) or saline as previously described ${ }^{16}$. DCA $(25 \mathrm{mg} / \mathrm{kg} /$ day, Sigma) was administered daily via i.p. injection, starting from day 0 of CVB3 infection. Animals were anaesthetized with xylazine $(10 \mathrm{mg} / \mathrm{kg})$ and ketamine $(100 \mathrm{mg} / \mathrm{kg})$ prior to sacrifice by cervical dislocation at 7 days post infection and all organs were excised, weighed and taken for further histological and molecular analysis.

\section{Cell culture}

Rat neonatal ventricular cardiac myocytes were isolated by enzymatic dissociation from the hearts of 1- to 2-day old neonatal rats. NCMs were plated $1.0 \times 10^{6}$ on gelatinized 6 -well plates and cultured as described previously ${ }^{17}$. HL-1 cells were kindly provided by Dr. W. Claycomb (Louisiana State University, New Orleans, LA), cultured in Claycomb medium (supplemented with 10\% FBS, $0.1 \mathrm{mM}$ norepinephrine, $2 \mathrm{mM}$ L-glutamine, 100 units $/ \mathrm{ml}$ penicillin, and $100 \mu \mathrm{g} / \mathrm{ml}$ streptomycin) at $37^{\circ} \mathrm{C}$ and $5 \% \mathrm{CO}_{2}$.

\section{Messenger RNA expression array analysis}

Mouse VM samples expression was measured using Affymetrix Mouse Gene 1.0 ST Array. The human total RNA was hybridized to Illumina HumanHT-12 v3.0 Gene Expression arrays. Detailed information about array analyses is available in the methods section in the online data supplement. 


\section{Statistics}

Data were analysed according to the guidelines of Altman et al. using SPSS (SPSS Inc., Chicago, IL, USA). Non-parametric tests and unpaired t-tests were used when appropriate. Data are represented as the mean \pm SEM. A p-value of less than 0.05 was considered to be significant.

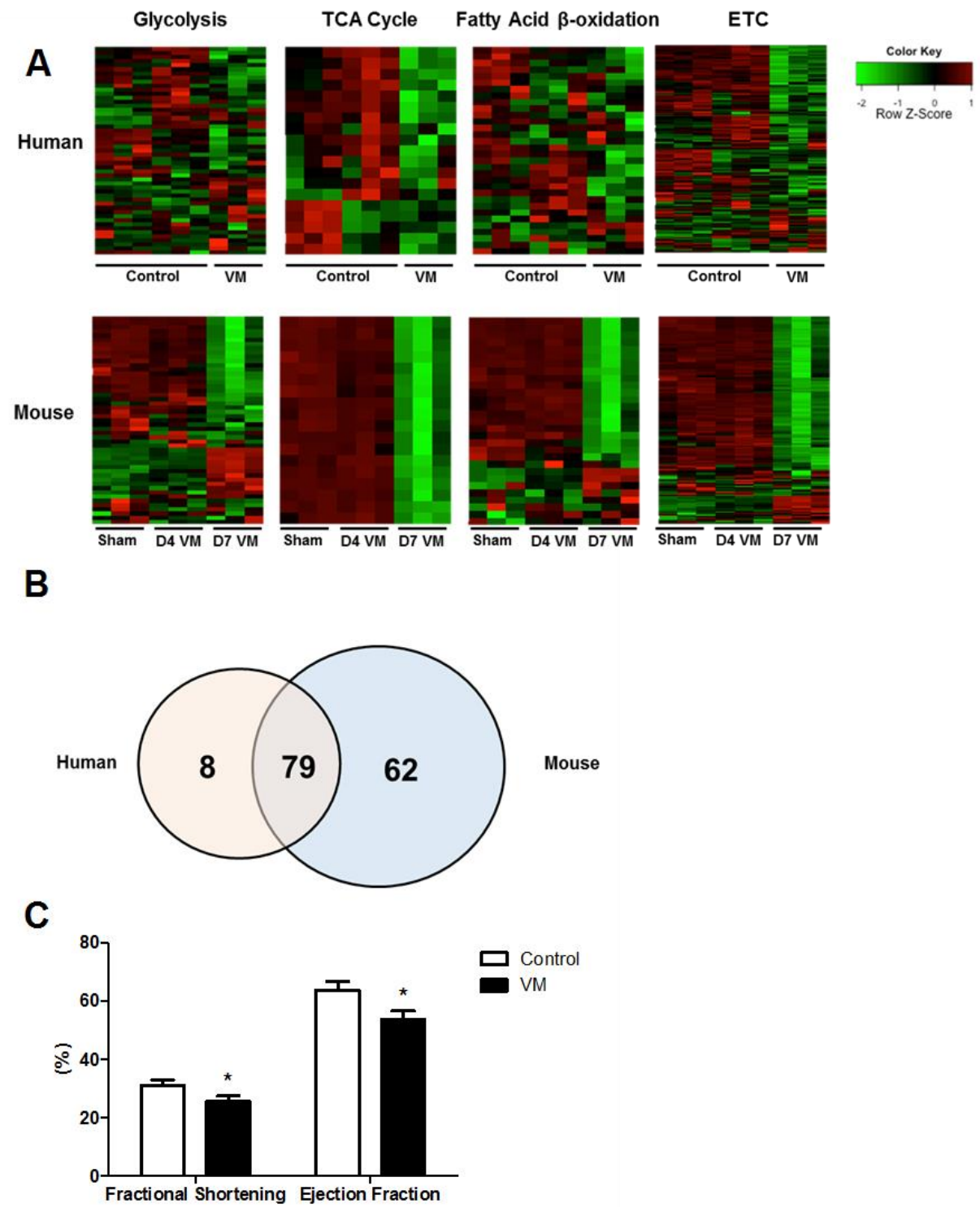

Figure 1. Micro-array analysis reveals down-regulation of oxidative metabolic pathways in the heart during VM. Heat maps of differentially-regulated ( $p \leq 0.05)$ metabolic genes in right ventricular septal biopsies from patients with viral myocarditis (VM) $(n=3)$ and controls $(n=6)$ and in cardiac tissue of mice with CVB3-induced VM, 4 and 7 days post inoculation, and sham-treated animals ( $n=3$ for all groups) (A). Venn diagram depicting overlap in differentiallyregulated metabolic genes in human and mouse VM (B). Echocardiographic assessment of the impact of VM (7 days post-inoculation) on cardiac function in mice (C). ${ }^{*} p \leq 0.05$ compared to control. 


\section{Results}

\section{Transcriptomic analysis reveals down regulation of oxidative metabolic pathways during}

acute VM

To identify cellular processes that are affected by VM in both humans and mice, micro-arrays were performed on cardiac biopsies of a previously well-characterized group of VM patients (Table S1) ${ }^{15}$ and on cardiac tissue of mice with acute CVB3-induced VM (Figure S1 A, B). Subsequent enrichment analysis revealed that processes related to cardiac energy metabolism were among the most prominently regulated processes in both man and mice (Table S2, S3). Accordingly, heat maps were constructed of all genes that are implicated in glycolysis, the tri-carboxylic acid (TCA) cycle, fatty acid $\beta$-oxidation and the electron transport chain (ETC). This revealed that, in the glycolytic pathway, a number of genes were upregulated while others were down-regulated in both human and mouse (Figure $1 \mathrm{~A})$. Remarkably, however, the far majority of genes involved in fatty acid $\beta$-oxidation, the TCA cycle and the ETC, was dramatically down-regulated during VM (Figure 1A; Visualization of metabolic pathways in human VM are depicted in Figure S2-S5). Significant overlap in differentially-regulated metabolic genes was observed between species (Fig 1B). Similar to observations in VM patients ${ }^{15}$, altered cardiac metabolic gene expression during CVB3induced VM was associated with a reduced ejection fraction (EF) and a decreased fractional shortening (FS) in absence of cardiac dilatation (Table S1 and Figure 1C). Notably, in mice, reductions in expression levels of constituents of mitochondrial oxidative metabolic pathways were much more pronounced at day 7 post-inoculation compared to day 4 (Figure $1 \mathrm{~A})$.

\section{VM-induced metabolic remodelling of the heart}

To validate our micro-array data, next we investigated mRNA transcript levels of several genes involved in cardiac oxidative metabolism in a mouse model of CVB3-induced VM and, importantly, subsequently verified whether alterations in the expression levels of these genes were associated with alterations in protein abundance and enzymatic activity of constituents of these metabolic pathways. As depicted in Figure $2 A$, enzymatic activity of $\beta$ hydroxyacyl-CoA dehydrogenase (HAD; rate-limiting enzyme in fatty acid $\beta$-oxidation) as well as cardiac mRNA levels of acyl-CoA dehydrogenase for very long chain fatty acids (ACAD-VL) were significantly decreased in VM confirming our micro-array data and further indicating a reduced fatty acid oxidative capacity. Moreover, VM was associated with significant impairments in mitochondrial oxidative phosphorylation as enzyme activity of cytochrome $c$ oxidase (COX) as well as protein and mRNA abundance of several sub-units of complexes involved in oxidative phosphorylation were reduced compared to sham-treated animals (Figure 2B).

In addition to impairments in oxidative metabolic pathways, transcriptomic analysis also indicated an increase in the expression levels of various glycolytic genes in the heart during VM. This was confirmed by increased expression levels of glucose transporter 1 (Glut1 ) and of several glycolytic enzymes as assessed by QPCR (Figure 2C). Also, lactate dehydrogenase-1 (LDH1) and the lactate transporter monocarboxylate transporter-4 (MCT-4) were increased expressed during CVB3-induced VM further evidencing activation of a glycolytic gene program (Figure 2D). Notably, transcript abundance of hypoxia-inducible factor $1 \alpha$ (HIF-1 $\alpha$ : a pivotal regulator of cardiac glycolytic metabolism) and of known HIF-1 $\alpha$ target genes were increased in VM hearts (Figure 2E) which correlated positively with 
glycolytic gene expression (Table. S4). Collectively, these alterations in oxidative and glycolytic metabolic pathways observed during VM were associated with an increase in the phosphorylation status of the energy-sensing molecule adenosine monophosphate kinase (AMPK) suggesting an energy-deprived state of the heart (Figure 2F). In concert, these data indicate that VM-induced metabolic changes observed at the level of the entire transcriptome are reflected by parallel changes at the protein and enzyme activity level and reveal impairments in oxidative metabolism, which appear to be (partly) compensated by HIF-dependent activation of glycolysis.

\section{Altered mitochondrial dynamics in VM}

Considering the aberrant expression and activity of several mitochondrial proteins essentially involved in oxidative substrate metabolism, cardiac mitochondrial morphology was assessed by electron microscopy. Although we did not observe prominent changes in mitochondrial ultra-structure 7 days post infection, we did observe altered mRNA expression levels of mitochondrial fusion and fission genes at this time-point (Figure $3 \mathrm{~A}, \mathrm{~B}$ ). More specifically, mRNA levels of the mitochondrial fusion gene $1(\mathrm{mfn}-1)$ decreased by approximately $40 \%$, while transcript abundance of the mitochondrial fission gene FIS1 increased 2-fold suggesting potential alterations in mitochondrial dynamics. 
A

Fatty acid $\beta$-oxidation

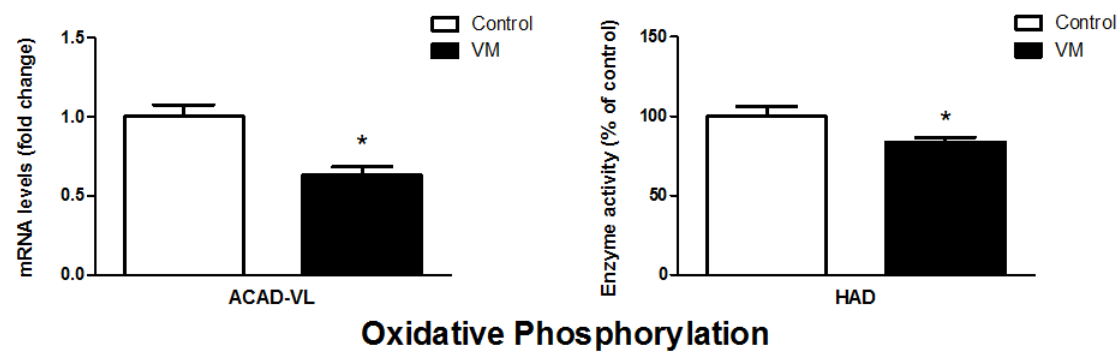

B
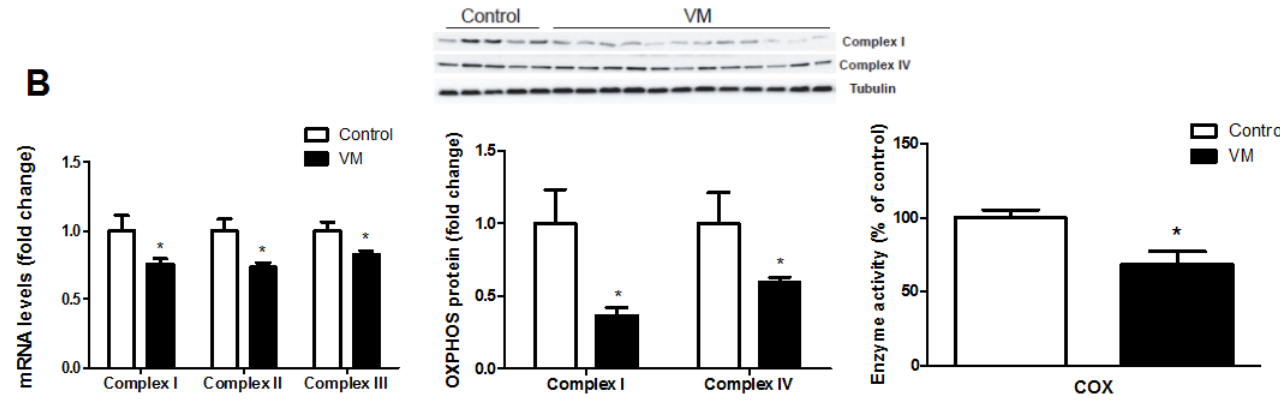

Glycolysis
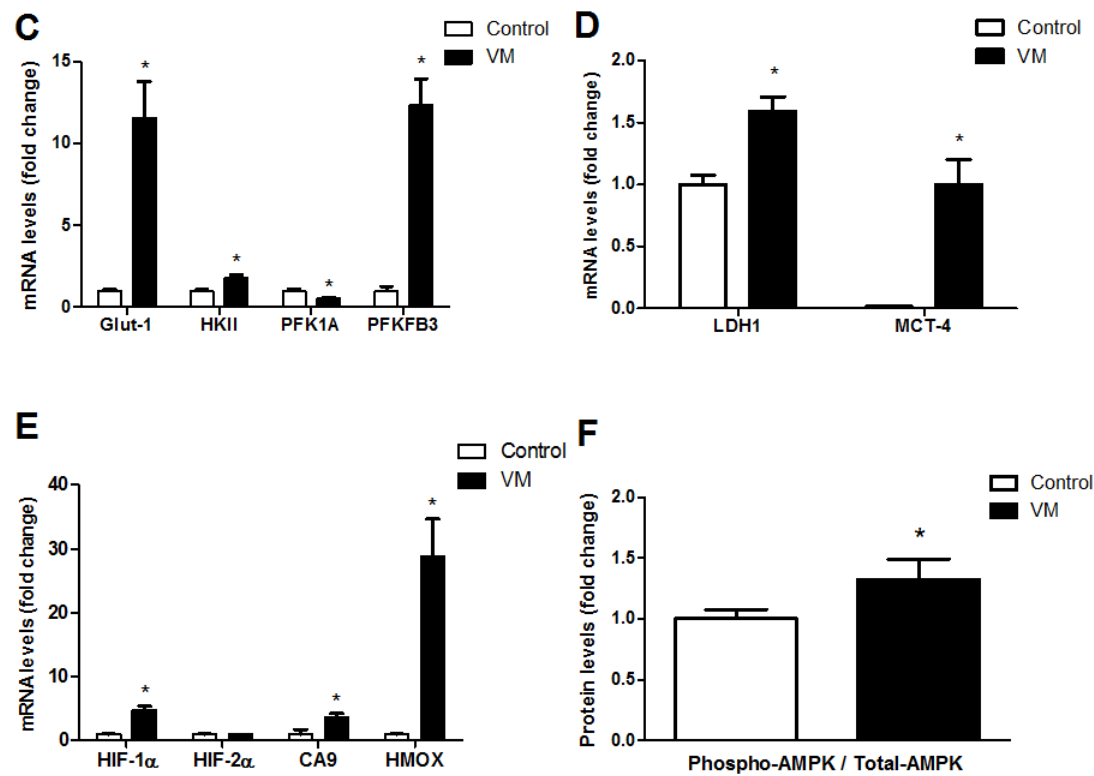

Figure 2. Down-regulation of mitochondrial oxidative metabolic pathways in the heart during CVB3-induced VM. Intact hearts were obtained from mice with CVB3-induced VM $(n=12)$ and sham-treated animals $(n=5)$. The activity of individual metabolic enzymes was determined and corrected for total protein content $(A, B)$. Western blot was performed to determine protein content of proteins of interest $(B, F)$ and metabolic gene expression was determined by QPCR (A-E). Western blots were corrected for $\alpha$-tubulin. ${ }^{*} p \leq 0.05$ compared to control. 
A
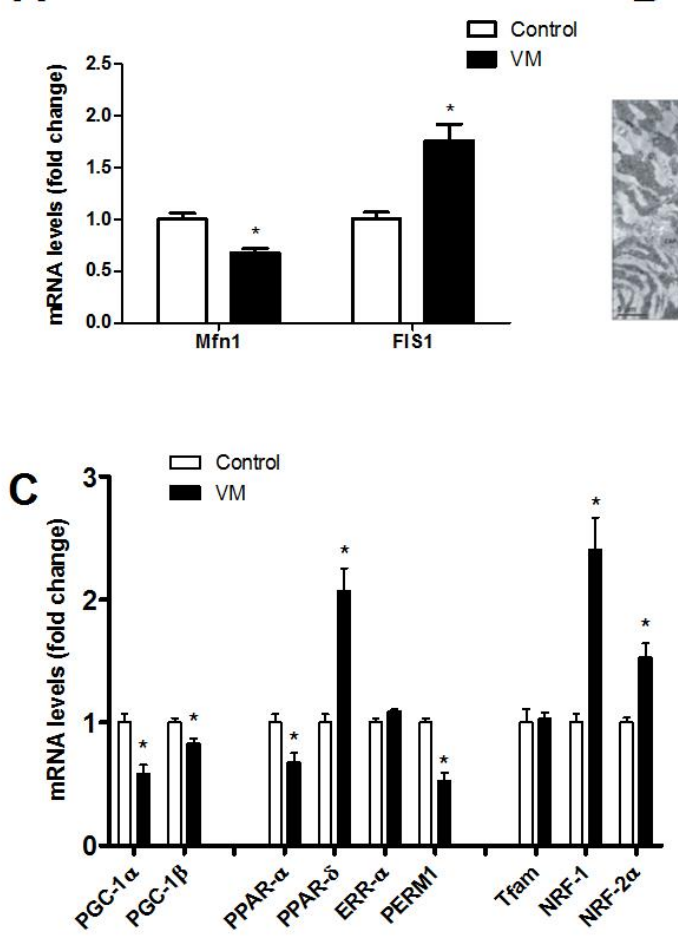

B

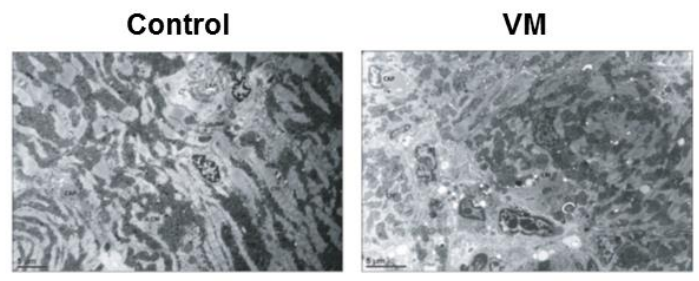

Figure 3. Disturbed mitochondrial dynamics and impaired PGC-1 signalling during CVB3-induced VM. C3H mice were subjected to CVB3-induced VM $(n=12)$ or sham-treated $(n=5)$ for 7 days. QPCR analysis revealed altered expression levels of mitochondrial fusion and fission genes (A). Electron microscopy images were taken from controls and animals with VM (B). Expression levels of constituents of the PGC-1 signalling network were altered during VM (C, D). Western blots were corrected for $\alpha$-tubulin. ${ }^{*} p \leq 0.05$ compared to control. Scale bar: $100 \mu \mathrm{M}$.

Peroxisome proliferator-activated receptor $\gamma$ co-activator molecule 1 (PGC-1) proteins are essential co-activators for a multitude of transcription factors involved in the molecular regulation of mitochondrial biogenesis and dynamics and hence are key determinants controlling cardiac oxidative metabolism ${ }^{18}$. Accordingly, PGC-1 $\alpha$ transcript abundance correlated positively with expression levels of various constituents of oxidative phosphorylation and fatty acid $\beta$-oxidation in our study (Table S4). Moreover, mRNA levels of PGC- $1 \alpha$ and of its family member PGC- $1 \beta$ were significantly reduced in the heart during VM as were expression levels of the PGC-1-coactivated transcription factors PPAR- $\alpha$ and PERM1. Protein levels of NRF-1 and Tfam, key regulators of mitochondrial biogenesis ${ }^{19}$, were dramatically decreased in VM further evidencing disturbances in the molecular regulation of mitochondrial metabolism. Interestingly, abundance of other PGC-1-co-activated transcription factors was unaltered (ERR- $\alpha$, Tfam) or increased (NRF-1, NRF-2 $\alpha$ and PPAR- $\delta$ ) (Figure $3 \mathrm{C}$-D) suggesting a compensatory cellular response to activate mitochondrial biogenesis. 
A

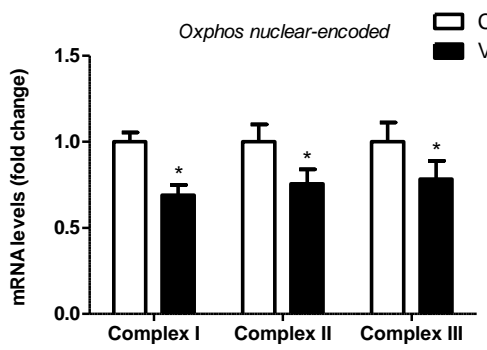

B

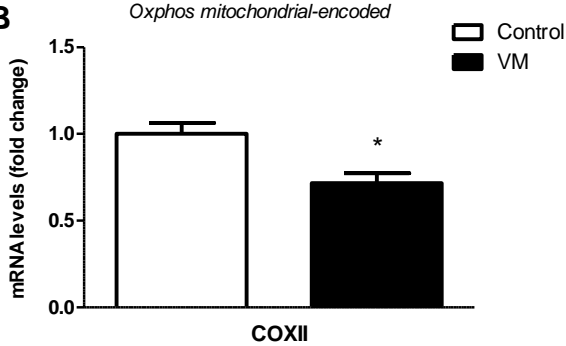

C

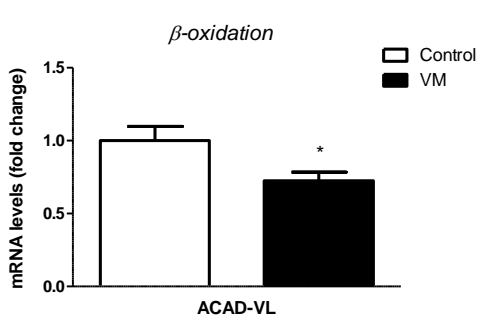

$\mathbf{E}$

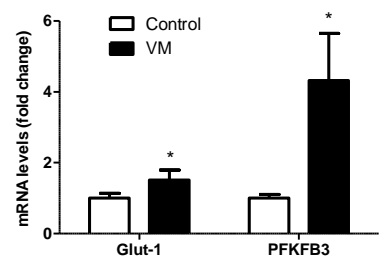

$\mathbf{F}$

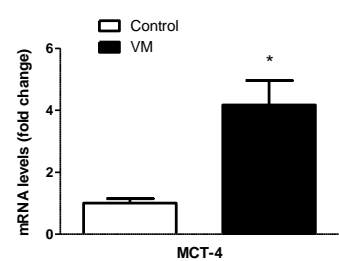

$\square$ Control

VM

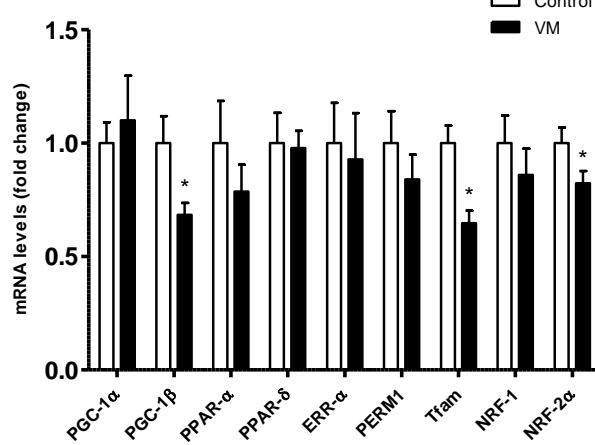

G

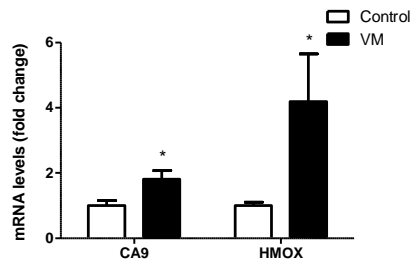

Figure 4. Cardiomyocyte metabolic gene expression in VM. Cardiomyocytes were isolated from hearts of mice with CVB3-induced VM $(n=8)$ and sham-treated animals $(n=8)$. QPCR analyses revealed down-regulation of genes involved in oxidative phosphorylation $(A, B)$, fatty acid B-oxidation $(C)$ and $P G C-1$ signalling $(D)$ while genes involved in glycolytic metabolism (E, F) and HIF signalling (G) were up-regulated. ${ }^{*} p \leq 0.05$ compared to control.

\section{Cardiomyocyte metabolic gene expression is impaired in VM}

Importantly, to verify whether the observed changes in metabolic pathways in intact hearts during VM reflect alterations in metabolic gene expression in cardiomyocytes per se and are not confined to infiltrating immune cells, cardiomyocytes were isolated from the hearts of animals with or without CVB3-induced VM. CVB3 viral RNA was detectable in cardiomyocytes isolated from animals with VM whereas control cardiomyocytes displayed no detectable levels (Figure S1C). In line with our in situ data, cardiomyocytes from VM animals were characterized by significantly reduced expression levels of nuclear- and mitochondrialencoded sub-units of oxidative phosphorylation complexes as well as decreased ACAD-VL (fatty acid $\beta$-oxidation) mRNA abundance (Figure $4 \mathrm{~A}-\mathrm{C}$ ). Furthermore, cardiomyocytes from animals with VM displayed decreased transcript levels of constituents of the PGC-1 signalling network as PGC-1 $\beta$ expression as well as Tfam and NRF-2 $\alpha$ mRNA levels were decreased (Figure 4D). Similar to what was observed in intact hearts, cardiomyocytes isolated from VM hearts also showed increased expression of multiple glycolytic constituents such as Glut-1, 
PFKFB3, and MCT-4 (Figure $4 \mathrm{E}, \mathrm{F}$ ). Although HIF-1 $\alpha$ expression itself was unaltered in cardiomyocytes isolated from VM hearts (data not shown), expression levels of the HIF-1 $\alpha$ target genes CA9 and HMOX were increased compared to control (Figure 4G).

\section{Inflammation-induced activation of NF-KB signalling impairs cardiomyocyte oxidative metabolism}

Having established a cardiomyocyte-specific metabolic remodelling process in VM, we next investigated potential underlying molecular mechanisms. Exposure of cultured neonatal cardiomyocytes to CVB3 virus potently induced expression of genes involved in viral uptake and host response to viral infection but failed to induce any significant decreases in oxidative metabolic gene expression (Figure S6A-E) suggesting that CVB3 virus infection itself does not impair cardiomyocyte metabolic gene expression.

Interestingly, in animals with VM, cardiac expression levels of the pro-inflammatory cytokine tumor necrosis factor $\alpha$ (TNF- $\alpha$ ) correlated inversely and significantly with expression levels of various genes involved in fatty acid $\beta$-oxidation, oxidative phosphorylation and PGC-1 signalling (Figure 5A-C), suggesting a role for TNF- $\alpha$ in VMinduced cardiac metabolic remodelling.

TNF- $\alpha$ is a known activator of the NF-KB pathway, a key inflammatory signalling cascade relaying inflammatory signals to the nucleus ${ }^{20}$. Our micro-array analysis revealed an up regulation in the expression levels of multiple constituents and target genes of the NF-KB pathway in cardiac biopsies of VM patients as well as in cardiac tissue of mice with VM, in particular 7 days post inoculation (Figure 5D). To confirm these micro-array data, expression levels of various inflammatory cytokines and chemokines, which are known NF-kB target genes ${ }^{21}$, were analysed by QPCR and found to be dramatically induced during CVB3-induced VM (Figure S7A). Moreover, cardiac expression levels of key constituents of NF-KB signalling itself $(\mathrm{IKB} \alpha, \mathrm{ReIA})$ were increased (Figure 5E) and correlated with the degree of cardiac immune cell influx (Table S4). Immunohistochemical staining for RelA, the main transcriptionally-active subunit of the NF-kB pathway, showed prominent NF-kB activation in the cardiomyocytes during CVB3-induced VM (Figure 5F). This was confirmed by increased expression levels of constituents and established target genes of the NF-KB pathway in cardiomyocytes isolated from the hearts of mice with VM (Figure S7B). 
A

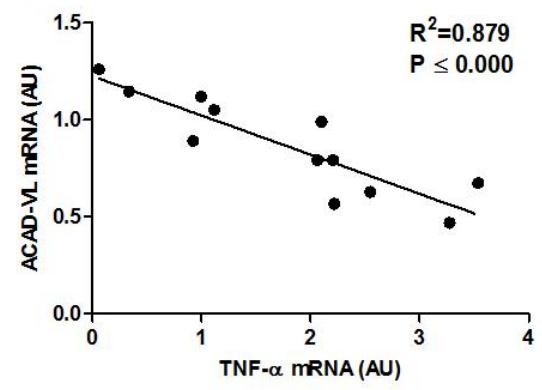

C

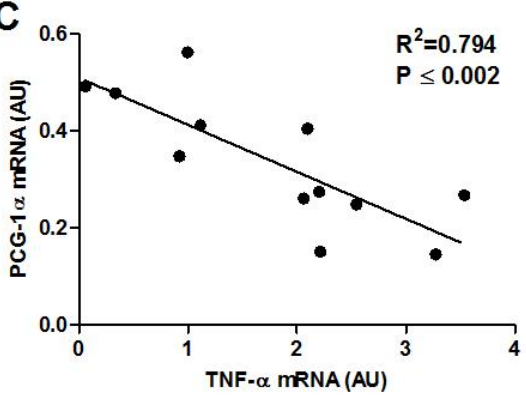

E

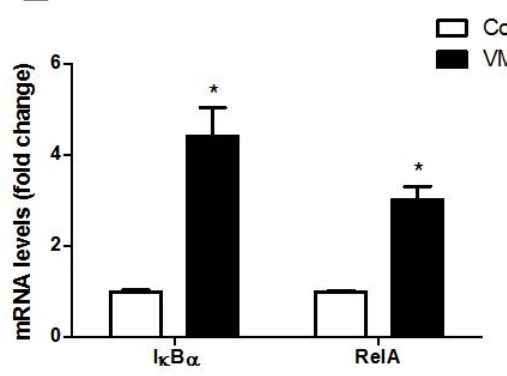

B
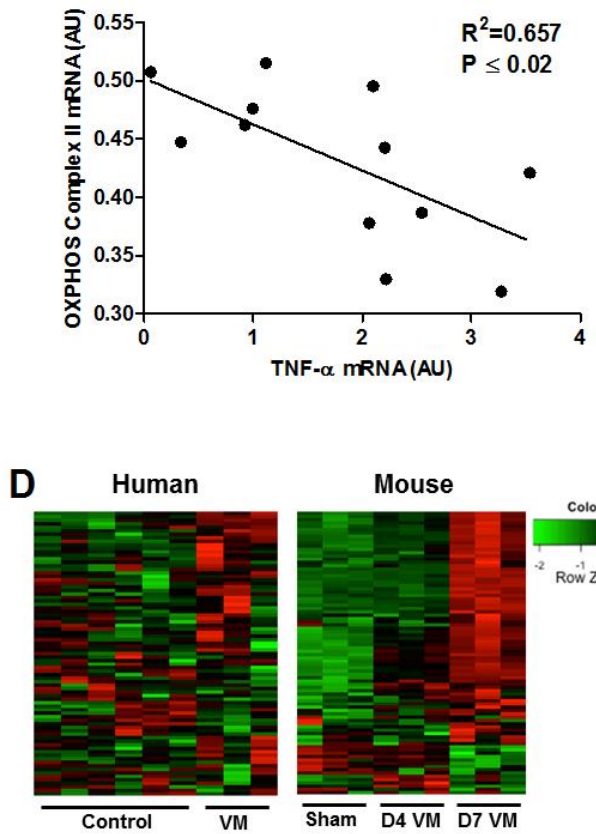

Color Key

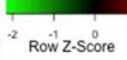

F
ReIA

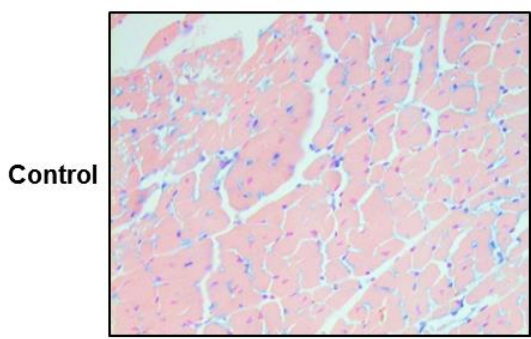

VM

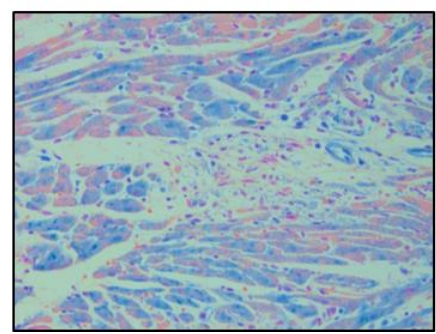

Figure 5. Activation of NF-KB signalling in cardiomyocytes during VM. Correlations between cardiac TNF- $\alpha$ expression and expression of constituents of fatty acid B-oxidation (A), oxidative phosphorylation (B) and PGC-1 signalling $(C)$ were explored in mice with CVB3-induced VM $(n=12)$. Heat map analysis revealed activation of $N F-K B$ signalling in right ventricular septal biopsies from patients with viral myocarditis (VM) (n=3) and in cardiac tissue of mice with CVB3-induced VM ( $n=3$ ) especially 7 days post-inoculation (D). Gene expression of NF- $K B$ signalling constituents was induced in cardiac tissue of mice with CVB3-induced VM ( $n=12)$ compared to sham-treated animals $(n=5)(E)$. Immuno-histochemical staining of RelA on sections from whole hearts from sham-treated mice $(n=5)$ and mice with CVB3-induced VM ( $n=9)$ demonstrated activation of NF-KB in cardiomyocytes (F). * $p \leq 0.05$ compared to control. 
To further explore a potentially direct detrimental impact of TNF- $\alpha$ and subsequent activation of the NF-KB pathway on cardiomyocyte oxidative metabolism, we stimulated cultured HL-1 cardiomyocytes with TNF- $\alpha$. In response to TNF- $\alpha$, inflammatory gene expression was induced (Figure S8A) and oxidative phosphorylation complex I, II and III subunit mRNA expression decreased significantly (Figure 6A). Moreover, TNF- $\alpha$ induced profound reductions in the expression levels of multiple constituents of the PGC-1 signalling network (Figure 6B) and decreased NRF-1 transcriptional activity $(84.4 \% \pm 2.8$ vs $100.0 \% \pm$ $5.1 ; \mathrm{P} \leq 0.05)$ and Tfam promoter transactivation $(86.6 \% \pm 4.8$ vs $100.0 \% \pm 4.7 ; \mathrm{P} \leq 0.05)$ indicating impairments in mitochondrial dynamics and biogenesis.

To examine whether NF-KB activation as such yielded similar results, we activated NF-KB signalling in cultured cardiomyocytes by adenoviral over-expression of IKK $\beta$, one of the key kinases involved in activation of the NF-KB pathway ${ }^{20}$. In HL-1 cardiomyocytes, NF-KB transcriptional activity as well as mRNA transcript abundance of intra-cellular adhesion molecule (ICAM-1), a known NF-KB target gene, was potently induced following IKK- $\beta$ overexpression (Figure $\mathrm{S} 8 \mathrm{~B}$ ). mRNA transcript abundance as well as protein content of oxidative phosphorylation complexes was found to be decreased in response to IKK $\beta$-induced NF-KB activation (Figure 6C, D). Also, IKK- $\beta$ over-expression was sufficient to decrease PGC-1 $\alpha$, PGC$1 \beta$, Tfam and PPAR- $\alpha$ transcript abundance similar to what was observed in response to TNF$\alpha$ (Figure 6E). IKK- $\beta$ over-expression induced similar changes in oxidative metabolic gene expression in cultured rat neonatal cardiomyocytes (Figure S8C-E).

In concert, this shows that (inflammation-induced) activation of the NF-KB signalling pathway impairs cardiomyocyte oxidative metabolic gene expression likely through interference with the PGC-1 signalling circuitry. This molecular mechanism may well be involved in VM-induced alterations in cardiac energy metabolism.

\section{DCA fails to restore VM-induced changes in cardiac metabolic gene expression}

Given the pronounced impairments in parameters of mitochondrial energy metabolism observed in acute VM, we explored whether systemic administration of DCA, a stimulator of glucose oxidation, would protect against VM-induced impairments in cardiac energy metabolism and thereby improve cardiac function. First, the effectiveness of DCA to upregulate cardiomyocyte oxidative metabolic gene expression was assessed in vitro using cultured neonatal cardiomyocytes. In line with previous studies ${ }^{22}$, the expression of PPAR- $\alpha$, involved in the regulation of cardiomyocyte oxidative metabolism, was up-regulated by DCA. Moreover, expression of a sub-unit of complex III of the respiratory chain was also upregulated indicating effectiveness of DCA (Figure 7A).

In vivo, VM resulted in an increased heart weight regardless of DCA treatment and DCA failed to protect against VM-induced cardiac dysfunction (Figure 7B, C). Surprisingly, DCA treatment of VM mice worsened the cardiac phenotype as evidenced by a marked increase in immune cell infiltration and inflammatory gene expression (Figure 7D) as well as more extensive metabolic remodelling at the mRNA level. Notably, spleen weight was also increased in DCA-treated versus vehicle-treated VM mice, suggestive of an exaggerated immune response (Figure $7 \mathrm{E}-\mathrm{G}$ ). 


\section{HL-1 cardiomyocytes}

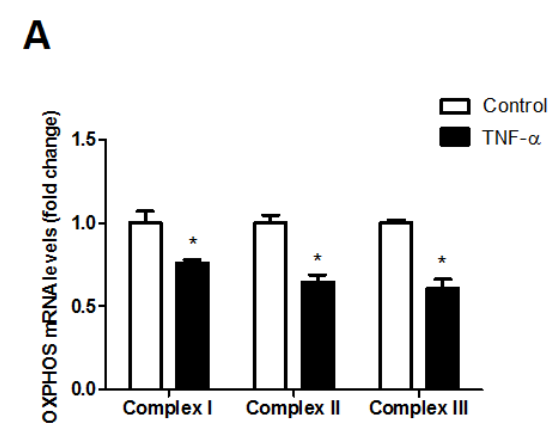

B

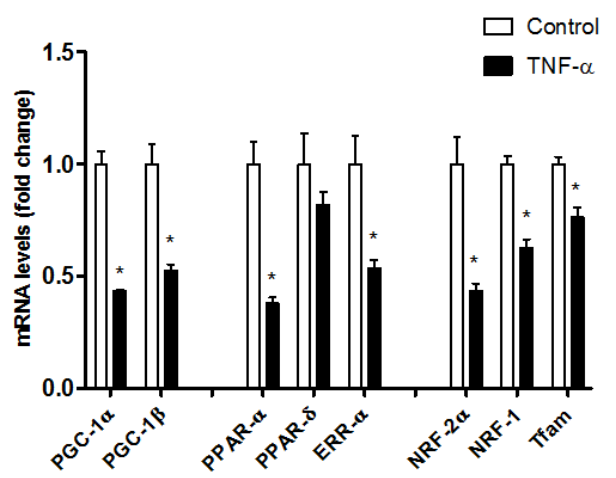

C

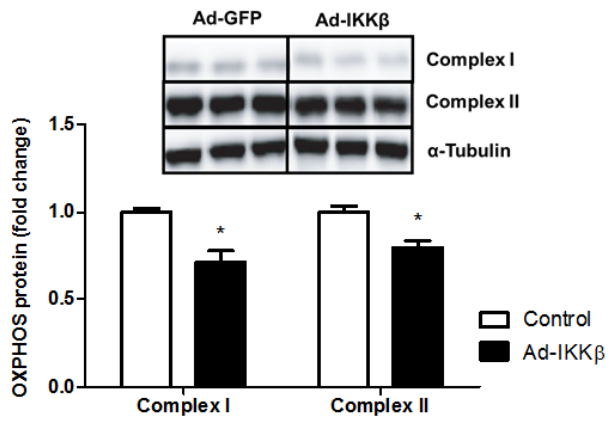

E
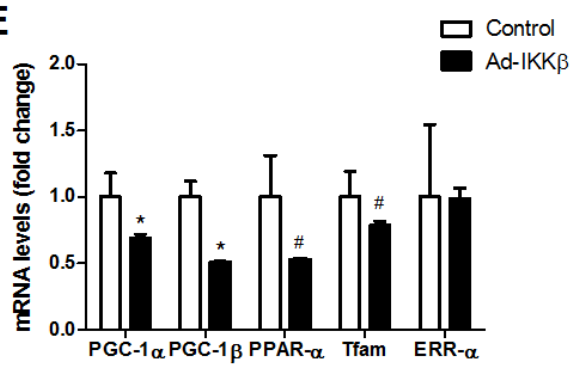

Figure 6. Activation of NF-KB signalling impairs cardiomyocyte oxidative phenotype. HL-1 cardiomyocytes were stimulated with $T N F-\alpha(10 \mathrm{ng} / \mathrm{ml})$ for $48 \mathrm{~h}$ and expression levels of genes involved in oxidative phosphorylation (A) and PGC-1 signalling (B) were determined. $H L-1$ cardiomyocytes were subjected to adenoviral over-expression of IKKB or an adenoviral construct expressing GFP as a control for $48 h(C, E)$ or $72 h(D)$. mRNA and protein expression levels of complexes of oxidative phosphorylation $(C, D)$ and $P G C-1$ signalling constituents $(E)$ were decreased. Western blots were corrected for $\alpha$-tubulin. Western blot triplicates for each treatment condition were cut from the same immunoblot as indicated by the black boxes. \# $p \leq 0.1, * p \leq 0.05$ compared to control. 
A

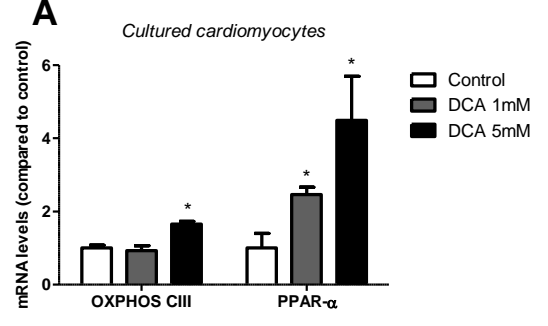

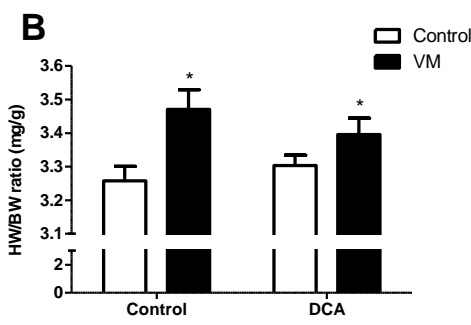

D
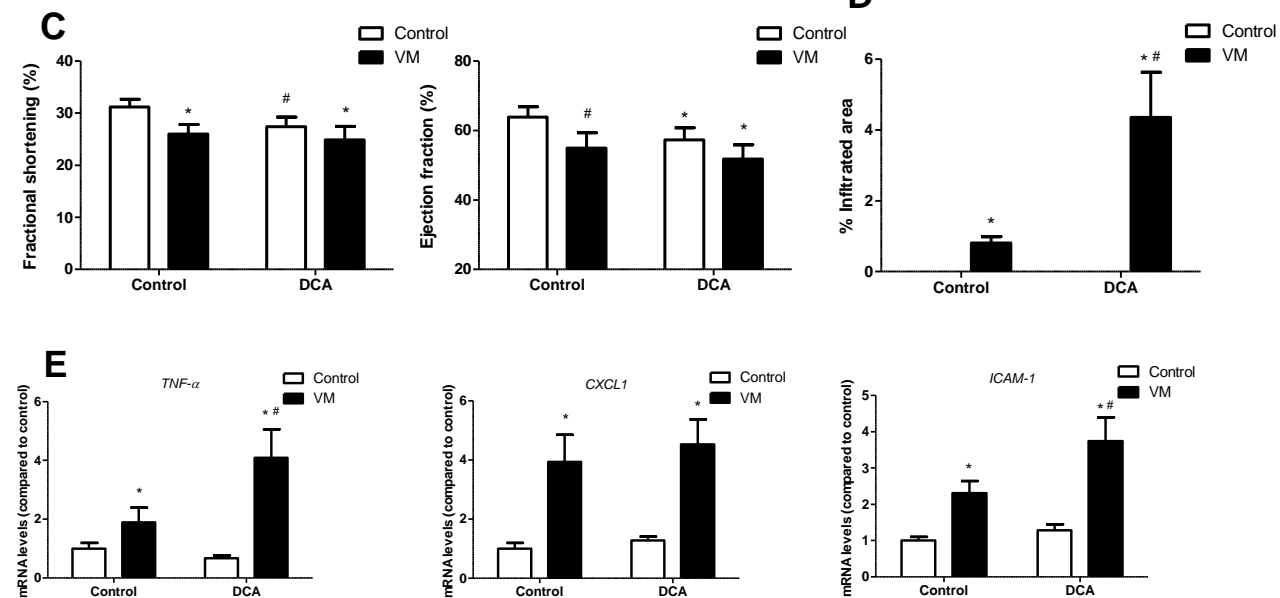

$\mathbf{F}_{\text {은 }}$
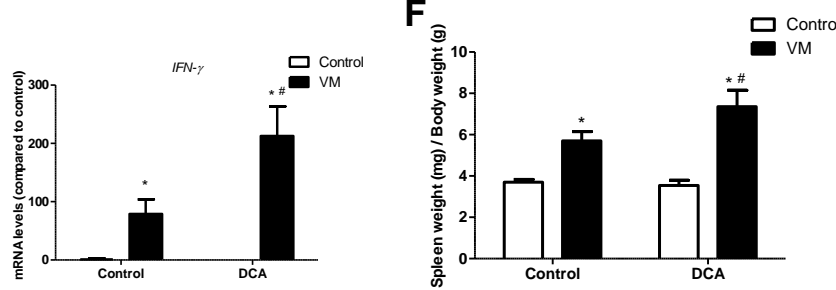

G
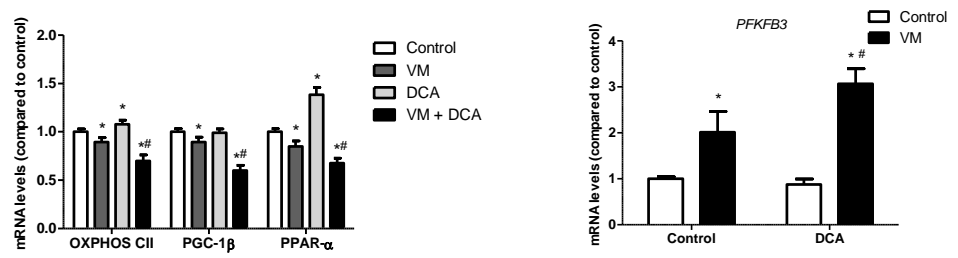

Figure 7. DCA aggravates VM-induced inflammation and fails to restore cardiac function and metabolic gene expression. Rat neonatal cardiomyocytes were cultured and stimulated with DCA for 24h. mRNA expression levels of PPAR- $\alpha$ and OXPHOS complex III sub-unit were analysed by QPCR (A). C3H mice received a single intraperitoneal (i.p) injection with CVB3 or vehicle. Additionally, all mice received an injection with vehicle or DCA (25 mg/ $\mathrm{kg} /$ day i.p) for 6 consecutive days. In VM animals, heart weight was increased and cardiac function was impaired regardless of DCA treatment $(B, C)$. DCA potentiated VM-induced increases in cardiac immune cell infiltrate and inflammatory gene expression $(D, E)$ as well as in spleen weight $(F)$. DCA potentiated VM-induced reductions in oxidative metabolic gene expression and inductions of glycolytic gene expression (G). ${ }^{*} p \leq 0.05$ compared to sham-control, ${ }^{*} p \leq 0.05$ compared to VM-control. 


\section{Discussion}

This is the first study showing that acute VM, in both humans and mice, is associated with metabolic remodelling of the heart. The present findings support the notion that the marked suppression of cardiomyocyte mitochondrial oxidative metabolism is linked to activation of the inflammatory NF-KB pathway, secondary to paracrine signalling by cytokines secreted by leukocytes infiltrating the myocardium and paracrine/autocrine signalling by inflammatory mediators produced by the cardiomyocytes themselves.

Changes in the energy metabolism of the heart and subsequent altered energetics, resulting in an 'energy-starved' heart, have been postulated to play an important causal role in the development of heart failure ${ }^{23}$. In humans and mice, we found striking similarities indicative for disturbed mitochondrial oxidative energy metabolism in the heart during VM which appeared to be (partly) compensated by activation of glycolysis. The increased phosphorylation status of the cellular energy-sensor AMPK revealed that metabolic remodelling during VM results in an energy-deprived state of the heart, which may well contribute to VM-induced cardiac dysfunction 23, 24. Besides contributing to cardiac dysfunction by providing an insufficient supply of ATP to cardiac myocytes, alterations in mitochondrial metabolic pathways may also have implications beyond ATP production as these pathways also regulate essential, non-ATP-generating anabolic processes that are crucial for maintaining cellular activities ${ }^{4}$. Importantly, alterations in metabolic gene expression in intact hearts were confirmed in cardiomyocytes isolated from the hearts of these animals, indicating that metabolic remodelling was confined to cardiomyocytes and not confounded by infiltrating immune cells which highly rely on glycolysis for energy generation ${ }^{25}$.

During VM, expression levels of TNF- $\alpha$ in the heart correlated negatively with abundance of key constituents and molecular regulators of mitochondrial oxidative metabolism, suggesting that inflammatory cytokines such as TNF- $\alpha$ may act as potential drivers of the cardiac metabolic remodelling process. The observation that stimulation of cultured cardiomyocytes with TNF- $\alpha$, but not exposure to CVB3 virus per se, potently impaired oxidative metabolic gene expression and its molecular regulation by PGC-1 signalling further strengthened this notion. As expression levels of TNF- $\alpha$ mRNA correlated strongly with the degree of cardiac immune cell influx, infiltrating immune cells likely are the main source of inflammatory cytokines in the heart during VM. However, cardiomyocytes themselves may well contribute to the ensuing inflammatory response in the heart as expression levels of various inflammatory cytokines were increased in cardiomyocytes isolated from hearts of animals with VM. These observations are in line with the observation that cardiac-specific over-expression of TNF- $\alpha$, a well-characterized model of cytokineinduced cardiomyopathy, resulted in mitochondrial dysfunction and impairments in cardiac oxidative metabolism ${ }^{26}$ and demonstrates, in line with other literature ${ }^{27}$ that TNF- $\alpha$ is a potent trigger for cardiomyocyte metabolic remodelling and development of cardiac dysfunction, relevant to VM-associated cardiac pathophysiology. Also, in addition to TNF- $\alpha$, cardiac-specific over-expression of other individual members of the TNF superfamily was sufficient to mediate adverse resulting in a dilated cardiac phenotype and mitochondrial dysfunction 28,29 suggesting a broader involvement of pro-inflammatory mediators and inflammatory signalling.

In this regard, studies have shown that RelA, the main transcriptionally-active sub-unit of the inflammatory NF-KB signalling pathway, not only impairs transcription of the PGC-1 $\alpha$ gene 
itself but also physically interacts with and sequester PGC-1 $\alpha$ protein thereby inhibiting its function in the regulation of cellular oxidative metabolism ${ }^{30-32}$. In addition, activation of NF$\mathrm{KB}$ signalling has been shown to impair transcription as well as activity of PPAR- $\alpha$ and PPAR- $\delta$ isoforms ${ }^{33-35}$. These studies further highlight the detrimental impact of activation of inflammatory signalling on the molecular regulation of oxidative energy metabolism and are in line with our findings as activation of NF-KB signalling in cardiomyocytes (in vitro as well as in vivo) was associated with reductions in expression levels of PGC-1 and PPAR isoforms. Interestingly, while Tfam and NRF-1 protein levels were decreased during VM, mRNA abundance of these key regulators of mitochondrial biogenesis increased suggesting a compensatory cellular response to activate mitochondrial biogenesis in response to cardiac energy depletion, a phenomenon also observed in experimental models of hypertrophic heart disease and heart failure ${ }^{36}$. Alterations in mitochondrial oxidative metabolic pathways and its molecular regulation by the PGC-1 network were observed within a few days of viral infection suggesting that perturbations in cardiac energy metabolism in VM do not arise secondary to a reduced cardiac function but likely contribute to impairment of cardiac function during disease progression ${ }^{37}$.

Illustrative of its significance, blockade of the NF-kB pathway improved cardiac function and survival during TNF- $\alpha$-induced cardiomyopathy and activation of NF-KB signalling, by cardiac-specific transgenic over-expression of IKK- $\beta$, was sufficient to induce foetal reprogramming of cardiomyocytes, cardiomyopathy and heart failure ${ }^{38,39}$. Together with our findings that impaired oxidative metabolism and disturbances in PGC-1 signalling during VM were associated with a massive induction of NF-KB signalling and that activation of NF-KB signalling per se was sufficient to impair cardiomyocyte oxidative gene expression, this suggests that NF-KB activation likely constitutes an important etiological factor involved in adverse cardiac metabolic remodelling observed in VM, most likely through downregulation of the PGC-1 signalling network. Intriguingly, NF-KB activation has also been shown in other experimental models of heart failure associated with cardiac metabolic remodelling ${ }^{40,41}$. In addition, NF-KB activation during CVB3-induced VM in our study was also associated with activation of a glycolytic gene program and increased expression of HIF-1 $\alpha$ and HIF-1 $\alpha$ target genes. This is in line with current understanding of these pathways as interactions between the inflammatory NF-KB pathway and the hypoxia-sensitive HIF-1 $\alpha$ pathway, which is known to regulate cardiac glycolytic gene expression ${ }^{42}$, have been described ${ }^{43-46}$.

Collectively, these observations are in line with the notion that, besides its well established role in regulating immune and inflammatory responses, the NF-KB pathway is increasingly being recognized as a central regulator of energy homeostasis via direct interaction with cellular networks that govern glycolysis and respiration ${ }^{47}$.

Given the marked derangements in cardiac energy metabolism observed in the present study and the notion that previous studies showed that correcting cardiac metabolism may offer relief to the diseased heart, we tried to stimulate oxidative metabolism in VM mice directly. Recently, Kato et al. demonstrated that DCA, an activator of the pyruvate dehydrogenase ( $\mathrm{PDH}$ ) complex and, hence, glucose oxidation, improved cardiac function and survival in a rat model of hypertensive heart disease ${ }^{48}$. Unexpectedly, the current study shows that in the setting of acute VM direct stimulation of mitochondrial metabolism with DCA worsened the VM phenotype as reflected by increased leukocyte infiltration, further reductions in metabolic oxidative gene expression and diminished cardiac function. Although the outcome is negative in terms of therapy, this experiment once again 
shows the intricate link between inflammation and metabolism ${ }^{47}$. Notably, administration of DCA to animals with VM also increased spleen weight indicative of an immune-modulatory effect of DCA. This is in coherence with available literature showing that DCA modulates cytokine response toward T-helper 1 lymphocyte function and is able to promote an M1 inflammatory phenotype in macrophages ${ }^{49}, 50$. Collectively, these findings suggest that the stimulation of glucose oxidation potentiates pro-inflammatory immune responses and in this way impairs anti-viral responses in VM and further impairs cardiomyocyte oxidative metabolism.

However, it cannot be excluded that DCA also exerts detrimental effects on cardiomyocytes in which oxidative metabolism is markedly affected, as seen in our VM model. It is feasible that stimulating PDH activity with DCA while respiratory chain activity is severely impaired further promotes rather than alleviates mitochondrial dysfunction. This would imply that metabolic therapy in VM demands that metabolism is stimulated in a more balanced way, for instance by stimulating the activity of more upstream regulators of cardiac metabolism, such as PGC-1, either directly or via the targeted inhibition of the NF-KB pathway. Inhibition of NF-KB signalling has already been shown to improve outcome in experimental models of myocarditis by attenuating inflammatory responses in the heart and inhibiting viral replication in lymphoid cells ${ }^{51,52}$. It remains to be established, however, if this is through its anti-inflammatory or metabolic effects.

In conclusion, the collective findings support the concept that cytokines, including TNF- $\alpha$, released from inflammatory cells infiltrating the infected myocardium, lead to the activation of NF-KB within the cardiomyocyte, ultimately resulting in impairment of mitochondrial oxidative metabolism. It is well feasible that these disturbances in energy generating capacity significantly contribute to the observed depression of cardiac contractile function during acute VM.

\section{Acknowledgements}

The authors would like to thank K. Custers for excellent technical assistance. This work was made possible by a VENI grant (016.126.044) from the Netherlands Organization for Scientific Research (to A.R) and by the Scientific Research Fund of Flanders (FWO) to AP and SH (G074009N). 


\section{References}

1. Esfandiarei M and McManus BM. Molecular biology and pathogenesis of viral myocarditis. Annu Rev Pathol. 2008;3:127-55.

2. Dennert R, Crijns HJ and Heymans S. Acute viral myocarditis. Eur Heart J. 2008;29:2073-82.

3. Kuhl U, Pauschinger M, Schwimmbeck PL, Seeberg B, Lober C, Noutsias M, Poller W and Schultheiss HP. Interferon-beta treatment eliminates cardiotropic viruses and improves left ventricular function in patients with myocardial persistence of viral genomes and left ventricular dysfunction. Circulation. 2003;107:2793-8.

4. Doenst T, Nguyen TD and Abel ED. Cardiac metabolism in heart failure: implications beyond ATP production. Circ Res. 2013;113:709-24.

5. van Bilsen $M$, van Nieuwenhoven FA and van der Vusse GJ. Metabolic remodelling of the failing heart: beneficial or detrimental? Cardiovascular research. 2009;81:420-8.

6. Kolwicz SC, Jr., Purohit S and Tian R. Cardiac metabolism and its interactions with contraction, growth, and survival of cardiomyocytes. Circ Res. 2013;113:603-16.

7. Doehner W, Frenneaux M and Anker SD. Metabolic Impairment in Heart Failure The Myocardial and Systemic Perspective. J Am Coll Cardiol. 2014;64:1388-1388.

8. Kato T, Niizuma S, Inuzuka Y, Kawashima T, Okuda J, Tamaki Y, Iwanaga Y, Narazaki M, Matsuda T, Soga T, Kita T, Kimura $T$ and Shioi T. Analysis of metabolic remodelling in compensated left ventricular hypertrophy and heart failure. Circ Heart Fail. 2010;3:420-30.

9. Alvarez-Guardia D, Palomer X, Coll T, Davidson MM, Chan TO, Feldman AM, Laguna JC and VazquezCarrera M. The p65 subunit of NF-kappaB binds to PGC-1alpha, linking inflammation and metabolic disturbances in cardiac cells. Cardiovasc Res. 87:449-58.

10. Nishtala K, Phong TQ, Steil L, Sauter M, Salazar MG, Kandolf R, Kroemer HK, Felix SB, Volker U, Klingel K and Hammer E. Virus-induced dilated cardiomyopathy is characterized by increased levels of fibrotic extracellular matrix proteins and reduced amounts of energy-producing enzymes. Proteomics. 2011;11:4310-20.

11. Xu J, Nie HG, Zhang XD, Tian $Y$ and $Y u$ B. Down-regulated energy metabolism genes associated with mitochondria oxidative phosphorylation and fatty acid metabolism in viral cardiomyopathy mouse heart. Mol Biol Rep. 2011;38:4007-13.

12. Remels AH, Gosker HR, Bakker J, Guttridge DC, Schols AM and Langen RC. Regulation of skeletal muscle oxidative phenotype by classical NF-kappaB signalling. Biochimica et biophysica acta. 2013.

13. Smeets PJ, Teunissen BE, Planavila A, de Vogel-van den Bosch H, Willemsen PH, van der Vusse GJ and van Bilsen $M$. Inflammatory pathways are activated during cardiomyocyte hypertrophy and attenuated by peroxisome proliferator-activated receptors PPARalpha and PPARdelta. The Journal of biological chemistry. 2008;283:29109-18.

14. Papageorgiou AP, Swinnen M, Vanhoutte D, VandenDriessche T, Chuah M, Lindner D, Verhesen W, de Vries B, D'Hooge J, Lutgens E, Westermann D, Carmeliet P and Heymans S. Thrombospondin-2 prevents cardiac injury and dysfunction in viral myocarditis through the activation of regulatory T-cells. Cardiovasc Res. 2012;94:115-24.

15. Corsten MF, Papageorgiou A, Verhesen W, Carai P, Lindow M, Obad S, Summer G, Coort SL, Hazebroek M, van Leeuwen R, Gijbels MJ, Wijnands E, Biessen EA, De Winther MP, Stassen FR, Carmeliet P, Kauppinen S, Schroen B and Heymans S. MicroRNA profiling identifies microRNA-155 as an adverse mediator of cardiac injury and dysfunction during acute viral myocarditis. Circulation research. 2012;111:415-25.

16. Heymans S, Pauschinger M, De Palma A, Kallwellis-Opara A, Rutschow S, Swinnen M, Vanhoutte D, Gao F, Torpai R, Baker AH, Padalko E, Neyts J, Schultheiss HP, Van de Werf F, Carmeliet P and Pinto YM. Inhibition of urokinase-type plasminogen activator or matrix metalloproteinases prevents cardiac injury and dysfunction during viral myocarditis. Circulation. 2006;114:565-73.

17. De Windt LJ, Willemsen PH, Popping S, Van der Vusse GJ, Reneman RS and Van Bilsen M. Cloning and cellular distribution of a group II phospholipase A2 expressed in the heart. J Mol Cell Cardiol. 1997;29:2095-106.

18. Puigserver $\mathrm{P}$ and Spiegelman BM. Peroxisome proliferator-activated receptor-gamma coactivator 1 alpha (PGC-1 alpha): transcriptional coactivator and metabolic regulator. Endocr Rev. 2003;24:78-90.

19. Jornayvaz FR and Shulman GI. Regulation of mitochondrial biogenesis. Essays Biochem. 2010;47:69-84.

20. Hayden MS and Ghosh S. Regulation of NF-kappaB by TNF family cytokines. Semin Immunol. 2014;26:25366.

21. Pahl HL. Activators and target genes of Rel/NF-kappaB transcription factors. Oncogene. 1999;18:6853-66.

22. Zhou YC and Waxman DJ. Activation of peroxisome proliferator-activated receptors by chlorinated hydrocarbons and endogenous steroids. Environ Health Perspect. 1998;106 Suppl 4:983-8. 
23. Neubauer S. The failing heart--an engine out of fuel. N Engl J Med. 2007;356:1140-51.

24. Fukushima A, Milner K, Gupta A and Lopaschuk GD. Myocardial Energy Substrate Metabolism in Heart Failure : from Pathways to Therapeutic Targets. Curr Pharm Des. 2015;21:3654-64.

25. Newsholme P, Gordon S and Newsholme EA. Rates of utilization and fates of glucose, glutamine, pyruvate, fatty acids and ketone bodies by mouse macrophages. Biochem J. 1987;242:631-6.

26. Li YY, Chen D, Watkins SC and Feldman AM. Mitochondrial abnormalities in tumor necrosis factor-alphainduced heart failure are associated with impaired DNA repair activity. Circulation. 2001;104:2492-7.

27. Mariappan N, Elks CM, Fink B and Francis J. TNF-induced mitochondrial damage: a link between mitochondrial complex I activity and left ventricular dysfunction. Free Radic Biol Med. 2009;46:462-70.

28. Dai Y, Dent $P$ and Grant S. Tumor necrosis factor-related apoptosis-inducing ligand (TRAIL) promotes mitochondrial dysfunction and apoptosis induced by 7-hydroxystaurosporine and mitogen-activated protein kinase kinase inhibitors in human leukemia cells that ectopically express $\mathrm{Bcl}-2$ and $\mathrm{Bcl}-\mathrm{xL}$. $\mathrm{Mol}$ Pharmacol. 2003;64:1402-9.

29. Shi J, Jiang B, Qiu Y, Guan J, Jain M, Cao X, Bauer M, Su L, Burkly LC, Leone TC, Kelly DP and Liao R. PGC1alpha plays a critical role in TWEAK-induced cardiac dysfunction. PLoS One. 2013;8:e54054.

30. Alvarez-Guardia D, Palomer X, Coll T, Davidson MM, Chan TO, Feldman AM, Laguna JC and VazquezCarrera M. The p65 subunit of NF-kappaB binds to PGC-1alpha, linking inflammation and metabolic disturbances in cardiac cells. Cardiovasc Res. 2010;87:449-58.

31. Irrcher I, Ljubicic V, Kirwan AF and Hood DA. AMP-activated protein kinase-regulated activation of the PGC-1alpha promoter in skeletal muscle cells. PLoS One. 2008;3:e3614.

32. Palomer X, Alvarez-Guardia D, Rodriguez-Calvo R, Coll T, Laguna JC, Davidson MM, Chan TO, Feldman AM and Vazquez-Carrera M. TNF-alpha reduces PGC-1alpha expression through NF-kappaB and p38 MAPK leading to increased glucose oxidation in a human cardiac cell model. Cardiovasc Res. 2009;81:703-12.

33. Serrano-Marco L, Chacon MR, Maymo-Masip E, Barroso E, Salvado L, Wabitsch M, Garrido-Sanchez L, Tinahones FJ, Palomer X, Vendrell J and Vazquez-Carrera M. TNF-alpha inhibits PPARbeta/delta activity and SIRT1 expression through NF-kappaB in human adipocytes. Biochim Biophys Acta. 2012;1821:117785.

34. Palomer X, Alvarez-Guardia D, Davidson MM, Chan TO, Feldman AM and Vazquez-Carrera M. The interplay between NF-kappaB and E2F1 coordinately regulates inflammation and metabolism in human cardiac cells. PLoS One. 2011;6:e19724.

35. Rodriguez-Calvo R, Serrano L, Barroso E, Coll T, Palomer X, Camins A, Sanchez RM, Alegret M, Merlos M, Pallas M, Laguna JC and Vazquez-Carrera M. Peroxisome proliferator-activated receptor alpha downregulation is associated with enhanced ceramide levels in age-associated cardiac hypertrophy. J Gerontol A Biol Sci Med Sci. 2007;62:1326-36.

36. Aubert G, Vega RB and Kelly DP. Perturbations in the gene regulatory pathways controlling mitochondrial energy production in the failing heart. Biochim Biophys Acta. 2013;1833:840-7.

37. Enache I, Charles AL, Bouitbir J, Favret F, Zoll J, Metzger D, Oswald-Mammosser M, Geny B and Charloux A. Skeletal muscle mitochondrial dysfunction precedes right ventricular impairment in experimental pulmonary hypertension. Mol Cell Biochem. 2013;373:161-70.

38. Moe GW, Marin-Garcia J, Konig A, Goldenthal M, Lu X and Feng Q. In vivo TNF-alpha inhibition ameliorates cardiac mitochondrial dysfunction, oxidative stress, and apoptosis in experimental heart failure. Am J Physiol Heart Circ Physiol. 2004;287:H1813-20.

39. Maier HJ, Schips TG, Wietelmann A, Kruger M, Brunner C, Sauter M, Klingel K, Bottger T, Braun T and Wirth T. Cardiomyocyte-specific IkappaB kinase (IKK)/NF-kappaB activation induces reversible inflammatory cardiomyopathy and heart failure. Proc Natl Acad Sci U S A. 2012;109:11794-9.

40. Gordon JW, Shaw JA and Kirshenbaum LA. Multiple facets of NF-kappaB in the heart: to be or not to NFkappaB. Circ Res. 2011;108:1122-32.

41. Van der Heiden K, Cuhlmann S, Luong le A, Zakkar M and Evans PC. Role of nuclear factor kappaB in cardiovascular health and disease. Clin Sci (Lond). 2010;118:593-605.

42. Xue W, Cai L, Tan Y, Thistlethwaite P, Kang YJ, Li X and Feng W. Cardiac-specific overexpression of HIF1 \{alpha\} prevents deterioration of glycolytic pathway and cardiac remodeling in streptozotocin-induced diabetic mice. Am J Pathol. 2010;177:97-105.

43. Taylor CT. Interdependent roles for hypoxia inducible factor and nuclear factor-kappaB in hypoxic inflammation. J Physiol. 2008;586:4055-9.

44. Zhou J, Schmid T and Brune B. Tumor necrosis factor-alpha causes accumulation of a ubiquitinated form of hypoxia inducible factor-1alpha through a nuclear factor-kappaB-dependent pathway. Mol Biol Cell. 2003;14:2216-25.

45. van Uden P, Kenneth NS and Rocha S. Regulation of hypoxia-inducible factor-1alpha by NF-kappaB. Biochem J. 2008;412:477-84. 
46. Rius J, Guma M, Schachtrup C, Akassoglou K, Zinkernagel AS, Nizet V, Johnson RS, Haddad GG and Karin M. NF-kappaB links innate immunity to the hypoxic response through transcriptional regulation of HIF1alpha. Nature. 2008;453:807-11.

47. Tornatore L, Thotakura AK, Bennett J, Moretti M and Franzoso G. The nuclear factor kappa B signaling pathway: integrating metabolism with inflammation. Trends Cell Biol. 2012;22:557-66.

48. Kato T, Niizuma S, Inuzuka Y, Kawashima T, Okuda J, Tamaki Y, Iwanaga Y, Narazaki M, Matsuda T, Soga T, Kita T, Kimura T and Shioi T. Analysis of metabolic remodeling in compensated left ventricular hypertrophy and heart failure. Circulation Heart failure. 2010;3:420-30.

49. Ohashi T, Akazawa T, Aoki M, Kuze B, Mizuta K, Ito $Y$ and Inoue N. Dichloroacetate improves immune dysfunction caused by tumor-secreted lactic acid and increases antitumor immunoreactivity. Int J Cancer. 2013;133:1107-18.

50. Kumar A, Kant S and Singh SM. Antitumor and chemosensitizing action of dichloroacetate implicates modulation of tumor microenvironment: a role of reorganized glucose metabolism, cell survival regulation and macrophage differentiation. Toxicol Appl Pharmacol. 2013;273:196-208.

51. Watanabe R, Azuma RW, Suzuki J, Ogawa M, Itai A, Hirata Y, Komuro I and Isobe M. Inhibition of NFkappaB activation by a novel IKK inhibitor reduces the severity of experimental autoimmune myocarditis via suppression of T-cell activation. Am J Physiol Heart Circ Physiol. 2013;305:H1761-71.

52. Gui J, Yue Y, Chen R, Xu W and Xiong S. A20 (TNFAIP3) alleviates CVB3-induced myocarditis via inhibiting NF-kappaB signaling. PLoS One. 2012;7:e46515. 


\section{Supplemental methods}

\section{Messenger RNA expression array analysis}

Mouse VM samples mRNA expression was measured using Affymetrix Mouse Gene 1.0 ST Array. Expression was normalized using RNA from Affymetrix ${ }^{1}$ Bioconductor package and Ensembl mapped custom CDF (version 16) in R. The human total RNA was hybridized to Illumina Human miRNAv2 Expression Panel arrays by ServiceXS (Leiden, The Netherlands). The lumi ${ }^{2}$ package from Bioconductor was used to annotate and quantile normalize the expression data. To assess differential expression, the limma ${ }^{3}$ package was used and genes with an adjusted $p$-value of $<0.05$ were considered significantly differentially regulated. Pathway enrichment of the differentially regulated genes was done using the Statistics tool in PathVisio ${ }^{4}$ against the curated set of WikiPathway ${ }^{5}$ mouse pathways.

\section{Pathway visualization}

The presented pathways are adaptation of the following WikiPathway pathways: fatty acid $\beta$ oxidation (WP1269), glycolysis and gluconeogenesis (WP157) and TCA cycle (WP434). The electron transport chain (WP295) pathway was used as is. The adaptations focus on streamlining the enriched pathways. They are available on WikiPathways as: [new fatty acid], [new TCA] and [new glycolysis]. The visualization of expression data was done through PathVisio. Mouse to human gene mapping was obtained through the BioMart of Ensembl Release 75.

\section{Tissue preparation, Histology and Immunohistochemistry}

Directly after sacrifice the hearts were dissected, weighed and prepared for further analysis. Atria were removed from the isolated heart and the heart was cut in two pieces between base and apex. Transverse midsections of the hearts were fixed in zinc fixative (BD Pharmingen) for $48 \mathrm{~h}$ and later embedded in paraffin, sectioned at $5 \mu \mathrm{m}$, and later processed for hematoxylin / eosin (H\&E) staining. The amount of cardiac inflammation was determined by calculating the infiltrate area of 10 random photo's at $10 x$ magnification of an H\&E staining using ImageJ program. Paraffin sections were first deparaffinized and rehydrated. The slides were blocked in buffer containing normal Rabbit serum (Dako), incubated with primary antibody Anti-Mouse CD45 (BD Pharmingen), and then incubated with biotinconjugated secondary Rabbit anti-Rat antibody (Dako). Signal was amplified using $A B C$ kit (Vectastain, Vectorlabs, CA) and visualized using DAB (Sigma-Aldrich).

\section{RelA staining}

Paraffin sections were deparaffinized using xylene and rehydrated. Antigen retrieval was performed by boiling the sections which were subsequently blocked using $1 \%$ BSA. Sections were exposed to RelA antibody $1 / 500$ diluted in $0.1 \%$ BSA overnight at $4^{\circ} \mathrm{C}$. After 3 wash steps, a swine anti-rabbit IgG biotin labelled antibody (Dako, E0431) diluted 1/250 in 1\% BSA was applied for $1 \mathrm{~h}$ at room temperature. Signal was amplified using ABC kit (Vectastain, Vectorlabs, CA)

\section{Isolation of adult cardiomyocytes}

Adult mouse cardiac cells were extracted by enzymatic digestion using an adjusted method previously described ${ }^{6}$. Briefly, hearts were dissected and immediately perfused with calciumfree Tyrode solution via a Langendorf perfusion system. After enzymatic digestion 
(collagenase I, Worthington, UK) calcium was slowly reintroduced. Freshly isolated, adult cardiomyocytes were lysed directly in RNA lysis buffer for further molecular analysis.

\section{Echo analysis}

Mice were anesthetized (1\% isoflurane, ecuphar) and echocardiograpy was performed with a MS400 transducer (18-38 MHz) on a Vevo-imaging station (Vevo2100, VisualSonics). M-mode views, long and short axis B-mode views were obtained and heart rate and LV diameters at end-diastole, end-systole, anterior wall thickness, LV posterior wall thickness in end diastole, were measured, with which fractional shortening and ejection fraction was calculated.

\section{Chemicals and reagents}

TNF- $\alpha$ (Calbiochem, Nottingham, United Kingdom) was dissolved in $0.1 \%$ bovine serum albumin (BSA) which also served as a vehicle control ( $0.005 \%$ final concentration) to a stock of $200 \mathrm{ng} / \mathrm{ml}$. Pre-made adenoviral (AD) constructs (Ad-CMV-IKK $\beta$ and Ad-CMV-GFP) were purchased from Vector Biolabs (Philadelphia, USA). Viral expression constructs were dissolved in Dulbecco's Modified Eagle Medium (DMEM) with 2 \% BSA \& $2.5 \%$ Glycerol $\left(1 \times 10^{10} \mathrm{PFU} / \mathrm{ml}\right)$ and further diluted in sterile Hank's Balanced Salt solution (HBSS) (Invitrogen, Leusden, the Netherlands) to a stock concentration of $2 \times 10^{8} \mathrm{PFU} / \mathrm{ml}$. A final concentration of $2 \times 10^{7} \mathrm{PFU} / \mathrm{ml}$ was added to cells.

\section{RNA isolation, cDNA synthesis and QPCR}

Directly after sacrifice the hearts were dissected and the apex of the heart was sectioned in two parts and directly frozen in liquid nitrogen for RNA isolation. Left ventricular tissue was snap frozen in liquid nitrogen and processed for analysis of mRNA content by quantitative

PCR. For in vitro experiments cells were washed with phosphate-buffered saline (PBS) and lysed using RLT buffer (Qiagen, Venlo, the Netherlands) with added $1 \% \beta$-mercaptho-ethanol. Total RNA was isolated using RNeasy kit (Qiagen, Venlo, the Netherlands) following manufacturers protocol. cDNA was prepared using iScript kit (Biorad Inc., Hercules, CA, USA) using $1 \mu \mathrm{g}$ RNA of each sample as input. QPCR analysis was performed on a Biorad iCycler to determine the mRNA levels of the analysed genes.

\section{Electron Microscopy}

For determination of ultra-structural mitochondrial morphology, hearts were perfused and fixed with $2.5 \%$ glutaraldehyde in $0.1 \mathrm{M}$ phosphate buffer $(\mathrm{pH} 7.4)$. Post fixation was performed in $1 \%$ osmium tetroxide in $0.1 \mathrm{M}$ cacodylate buffer $(\mathrm{pH} 7.4)$ supplemented with $1.5 \%$ potassium ferrocyanide. The samples were then dehydrated and embedded in epon. Ultrathin sections were examined using a Philips CM100 electron microscope.

\section{Transfections and plasmids}

For the assessment of NF-KB transcriptional activity, cells were transfected with a $6 \mathrm{~KB}-\mathrm{TK}$ luciferase plasmid (NF-KB reporter) as previously described ${ }^{7}$. For assessment of Tfam promoter activity and NRF-1 transcriptional activity, cells were transfected with mTFARC4wt/PGL3 or p4xNRF-1 luciferase reporter construct ${ }^{7-9}$. Cells were grown to be $50 \%$ confluent and transfected by nanofectin according to manufacturer's instructions (PAA, Pasching, Austria). Cells were harvested by washing $2 x$ with cold PBS and subsequent lysis by adding $(100 \mu \mathrm{l}) 1 \mathrm{x}$ Reporter Lysis Buffer (Promega, Madison, WI, USA) and incubation on ice for $10 \mathrm{~min}$. Cell lysates were centrifuged (14000 rpm, $1 \mathrm{~min}$ ) and supernatants were snap 
frozen and stored at $-80{ }^{\circ} \mathrm{C}$ for later analysis. Luciferase activity was measured according to manufacturer's instructions (Promega) and corrected for $\beta$-galactosidase (Tropix, Bedford, MA, USA).

\section{Whole cell lysates and Western blot}

Whole heart was powdered and lysed in a whole-cell lysate (WCL) buffer (20 mM Tris, pH 7.4; 150 mM NaCl; 1 \% Nonidet P-40; 1 mM DTT; 1 mM Na3VO $; 1$ mM PMSF; 10 g/ml Leupeptin and $1 \%$ aprotenin) using a Polytron PT 1600 E (Kinematica AG, Littau/Luzern, Germany). HL-1 cells were harvested in the same buffer by scraping with rubber police men. Crude lysates were incubated on ice for $30 \mathrm{~min}$, followed by a $30 \mathrm{~min}$ centrifugation step at $16,000 \mathrm{~g}$ and 4 ${ }^{\circ} \mathrm{C}$. A portion of the supernatant was saved for protein determination, prior to the addition with $4 \times$ Laemmli sample buffer (0.25 M Tris-HCl pH 6.8; $8 \%$ (w/v) SDS; $40 \%$ (v/v) glycerol; $0.4 \mathrm{M} \mathrm{DTT}$, and $0.04 \%(\mathrm{w} / \mathrm{v})$ Bromophenol Blue). The samples were boiled for $5 \mathrm{~min}$ at $95{ }^{\circ} \mathrm{C}$ and stored at $-20{ }^{\circ} \mathrm{C}$. Total protein concentration was assessed with the Bio-Rad DC protein assay kit (Bio-Rad, Hercules, CA, USA) according to the manufacturer's instructions. For western blotting, $2.0 \mu \mathrm{g}$ (OXPHOS, GAPDH) or $10 \mu \mathrm{g}$ (NRF-1, Tfam, AMPK, phospho-AMPK, IKK- $\beta$ and $\alpha$-tubulin) of protein was loaded per lane and separated on a $10 \%$ polyacrylamide gel (Mini Protean 3 system; Biorad, Hercules, CA, USA), followed by transfer to a $0.45 \mu \mathrm{m}$ nitrocellulose membrane (Biorad) by electro-blotting. The membrane was blocked for $1 \mathrm{~h}$ at room temperature in $5 \%(\mathrm{w} / \mathrm{v})$ non-fat dry milk followed by overnight incubation $\left(4{ }^{\circ} \mathrm{C}\right)$ with a polyclonal antibody specific for OXPHOS proteins, $\alpha$-tubulin, NRF-1, Tfam, AMPK and phospho-AMPK (all from Cell Signaling, Beverly, MA, USA). Antibodies were all diluted 1/2000 in (w/v) non-fat dry milk. After three wash steps of 20 min each, the blots were probed with a peroxidase-conjugated secondary antibody (Vector Laboratories, Burlingame, CA, USA) and visualized using SuperSignal West Pico chemiluminescent substrate (Pierce Biotechnology, Rockford, IL, USA) according to the manufacturer's instructions. Western-blot films were imaged and quantified using the Quantity One analysis software from Bio-Rad.

\section{Enzyme activity assays}

Activity of $\beta$-hydroxy-acylCoA dehydrogenase (HAD) (EC 1.1.1.35) and cytochrome c oxidase (COX) (EC 1.9.3.1) were measured as described previously ${ }^{7,10}$. Enzyme activity levels were measured at $37^{\circ} \mathrm{C}$ and at $\mathrm{PH} 7.3$ and were corrected for total protein content which was determined using a detergent compatible protein determination assay (Bio-Rad). 


\section{Supplemental figures}
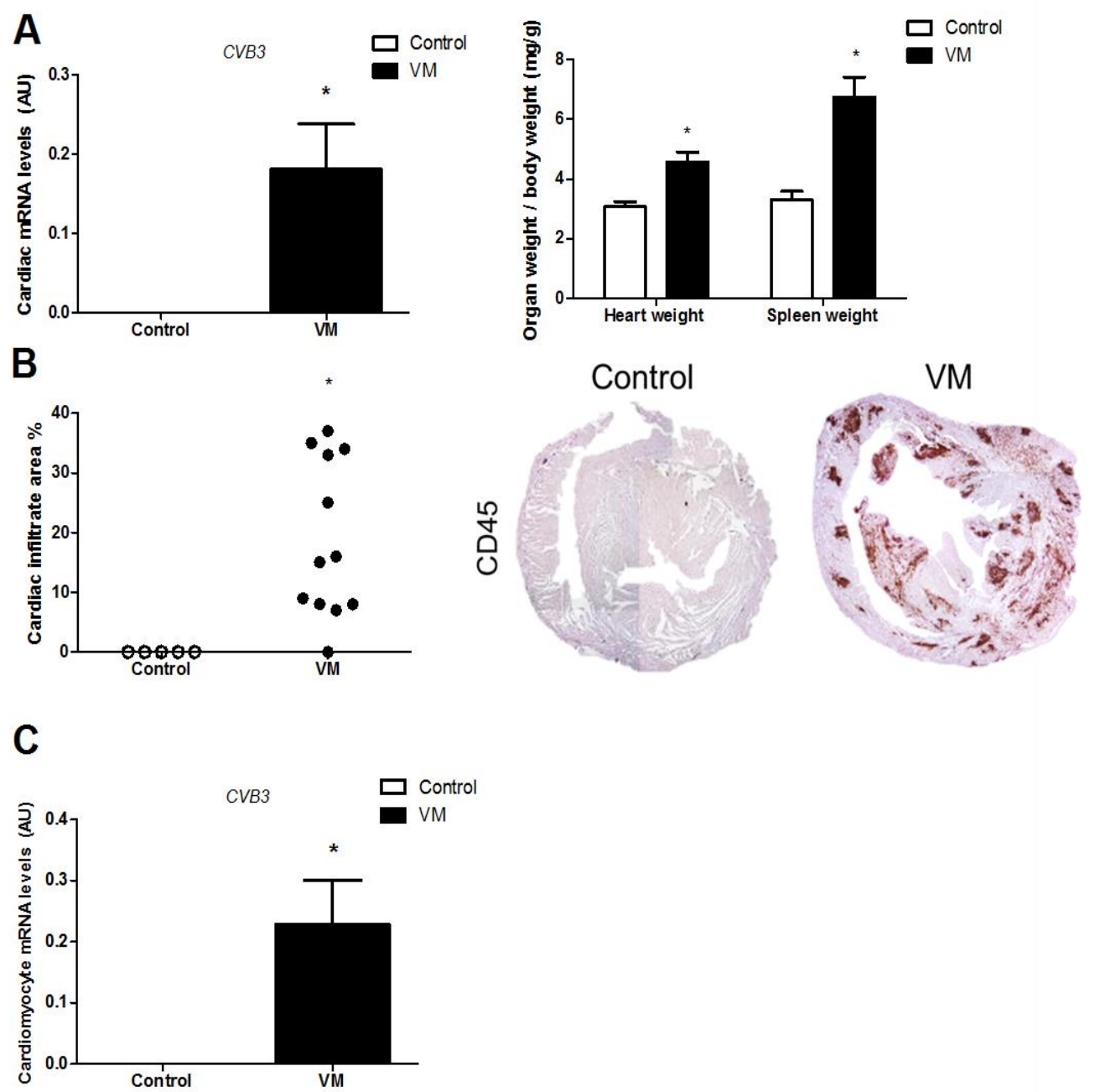

Figure S1. CVB3-induced VM in mice. C3H mice were subjected to CVB3-induced VM ( $n=12)$ or sham treatment $(n=5)$. The heart and spleen were collected and weighed and QPCR analysis was applied to determine viral load in the heart (A). Immunohistochemistry revealed massive cardiac immune cell influx (B). Cardiomyocytes were isolated from mice with CVB3-induced VM $(n=8)$ and sham-treated animals $(n=8)$ and CVB3 load was determined $(C){ }^{*} p \leq 0.05$ compared to control. 


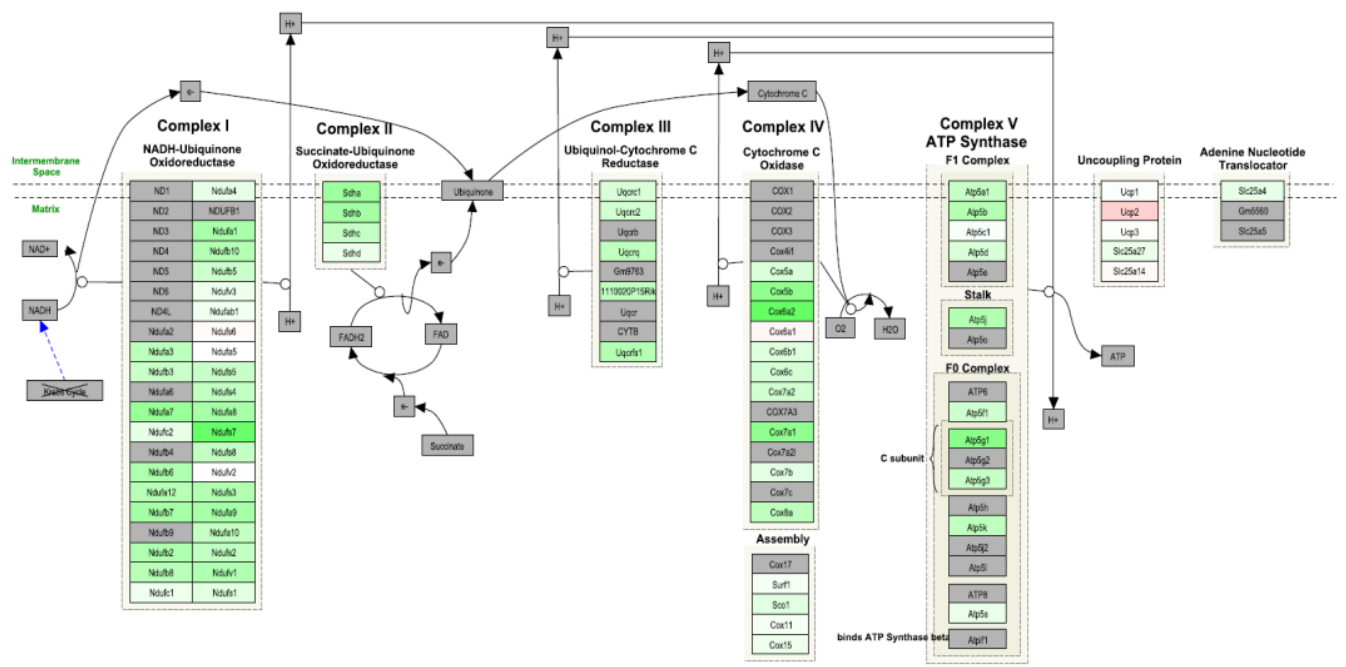

Figure S2. Pathway analysis of the electron transport chain in human VM. Red indicates upregulated and green indicates downregulated mRNA levels.

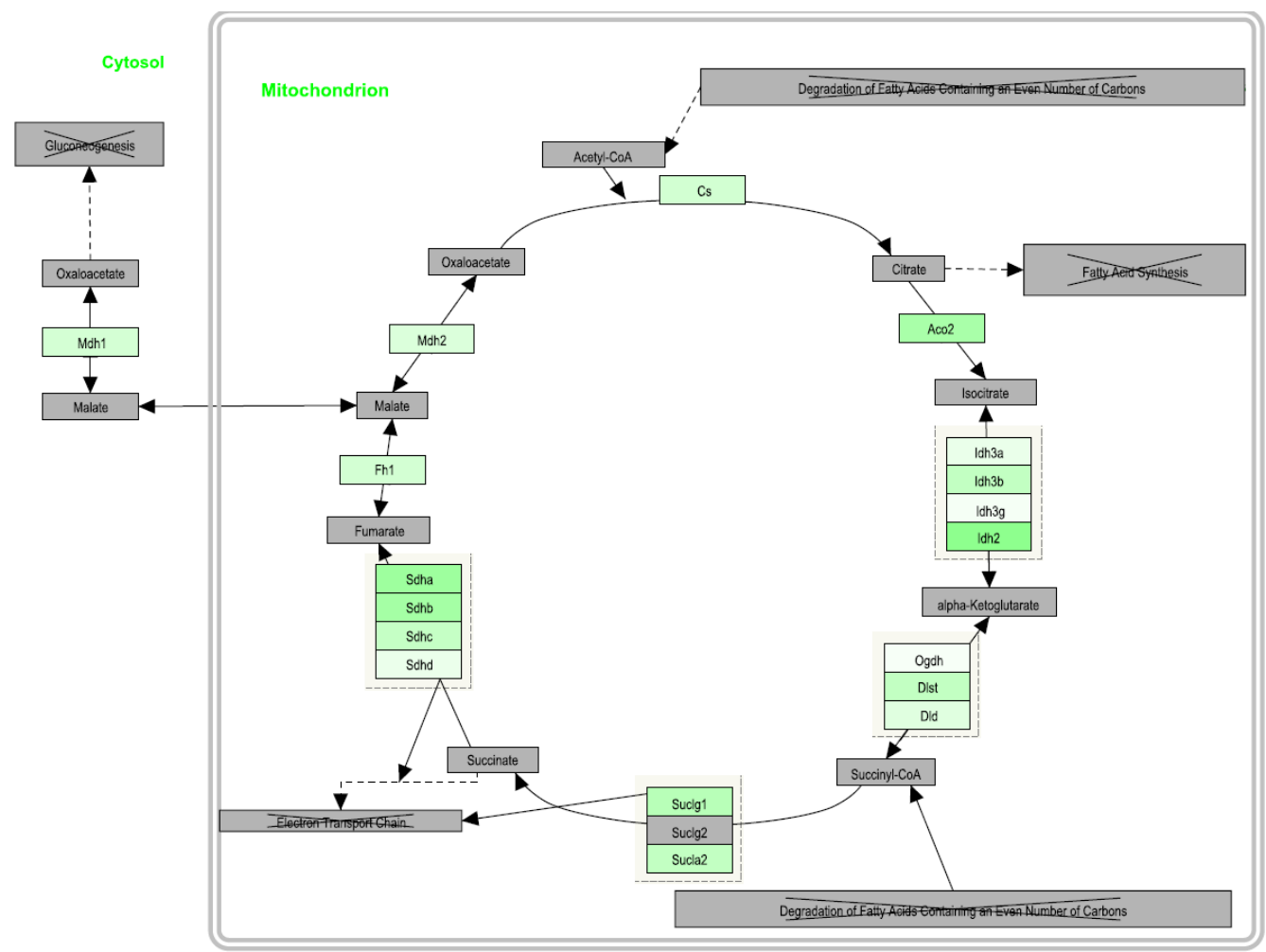

Figure S3. Pathway analysis of the TCA cycle in human VM. Red indicates upregulated and green indicates downregulated $m R N A$ levels.. 


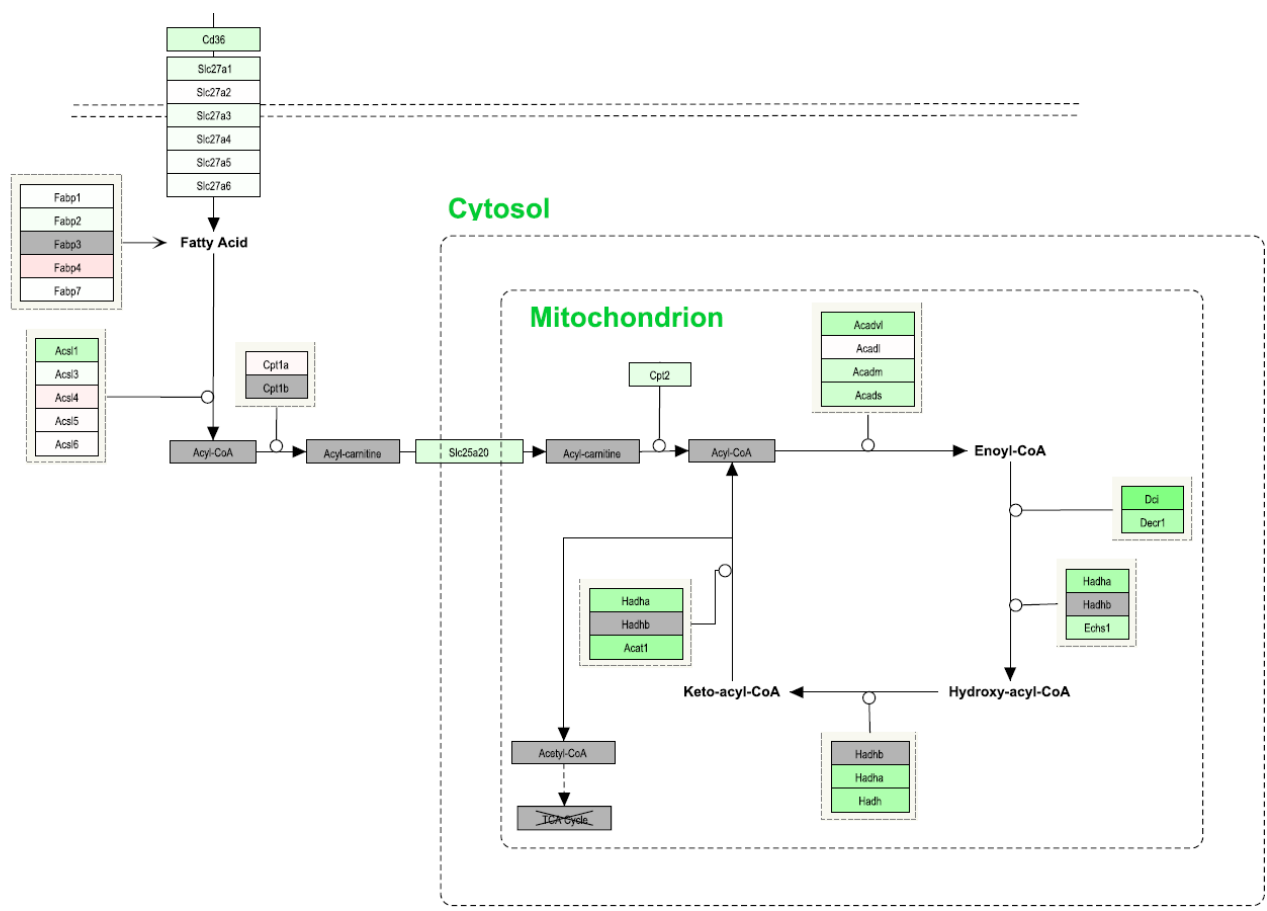

Figure S4. Pathway analysis of fatty acid $\beta$-oxidation in human VM. Red indicates upregulated and green indicates downregulated mRNA levels. 


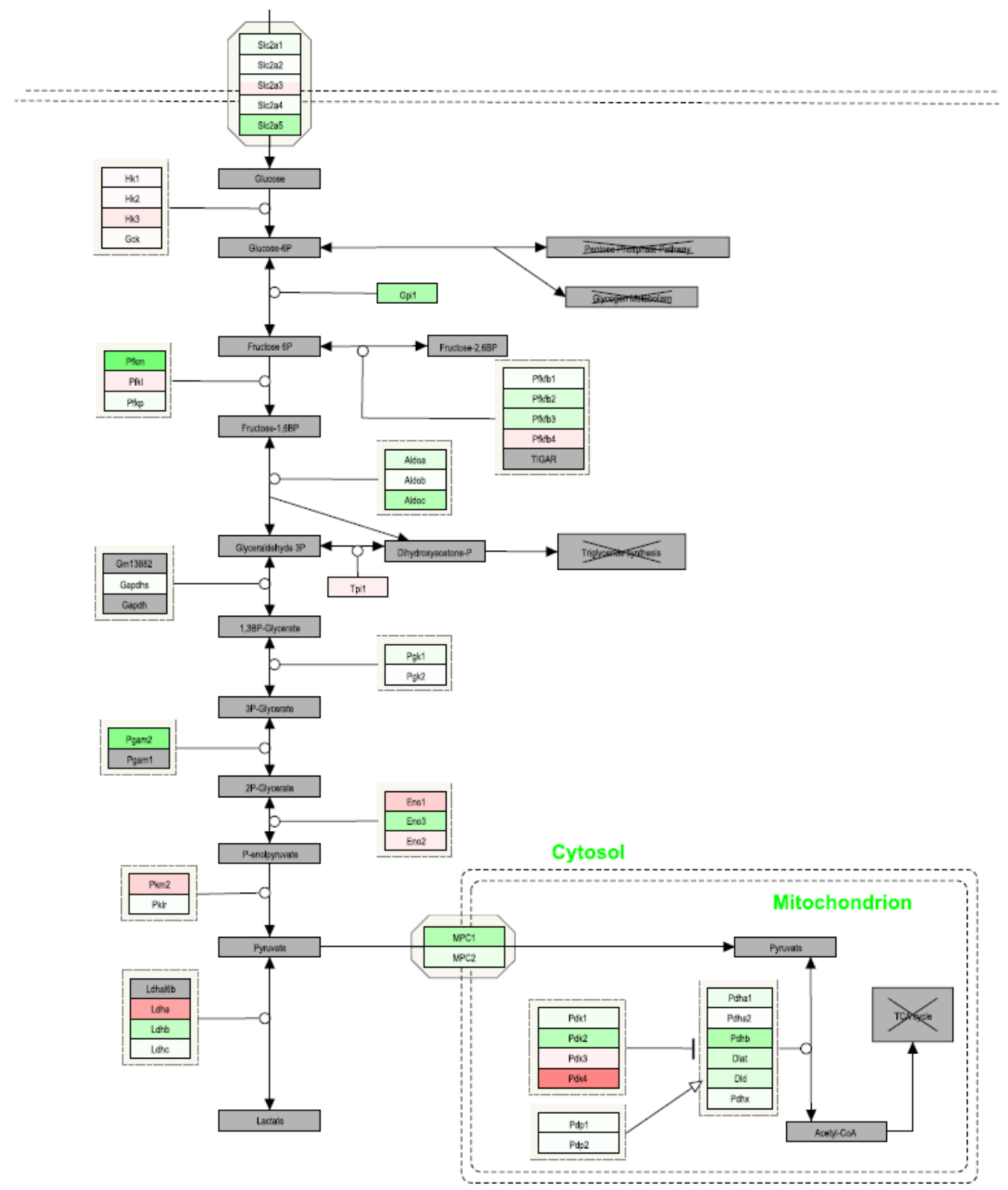

Figure S5. Pathway analysis of glycolysis in human VM. Red indicates upregulated and green indicates downregulated mRNA levels. 
A

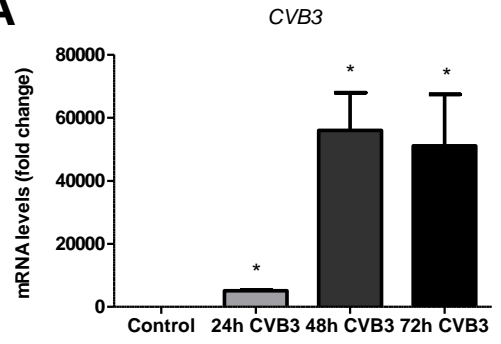

B

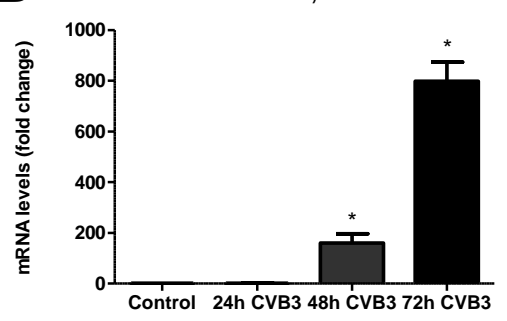

C

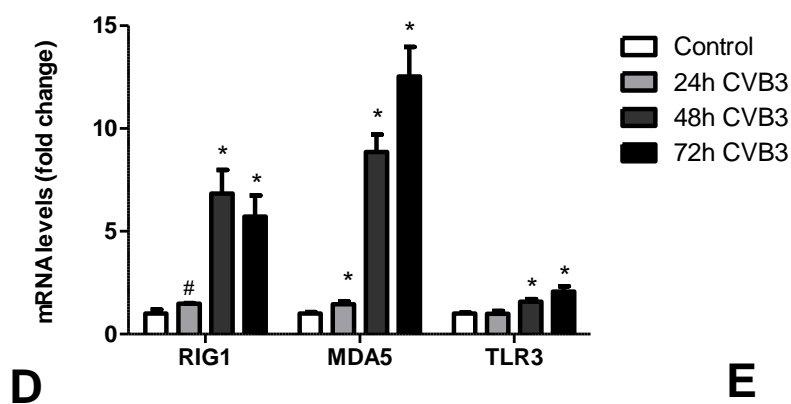

D
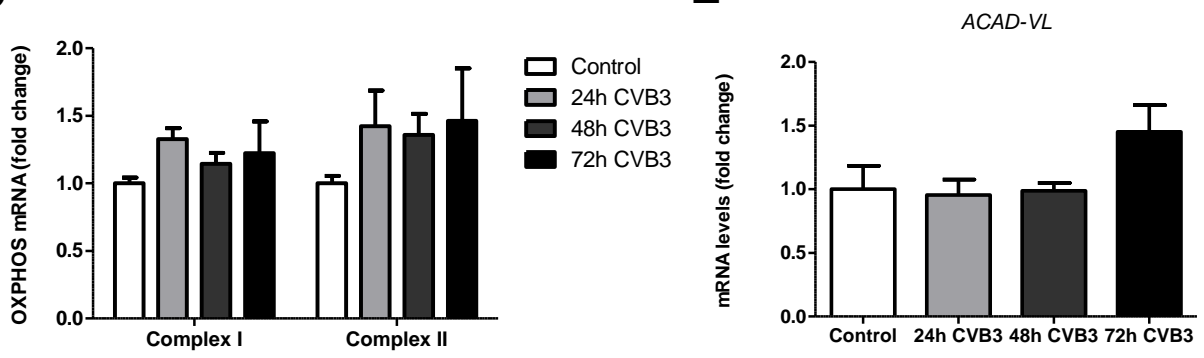

Figure S6. CVB3-induced gene expression in cardiomyocytes. Rat neonatal cardiomyocytes were cultured and stimulated with CVB-3 virus at a multiplicity of infection (MOI) of 1.0 for the indicated times. QPCR analyses revealed increased expression of genes involved in viral uptake and replication $(A-C)$ while genes involved in oxidative phosphorylation and fatty acid B-oxidation were unaltered or increased $(D, E){ }^{*} p \leq 0.05,{ }^{*} \leq 0.1$ compared to control. 

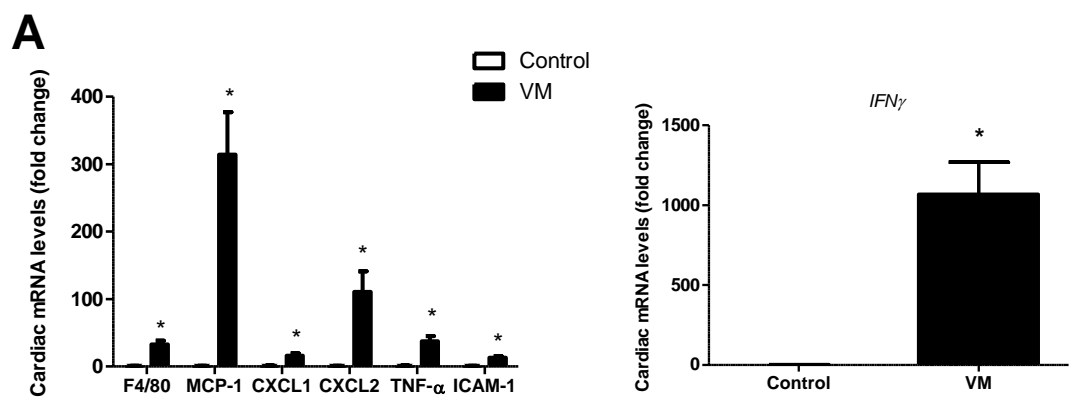

B
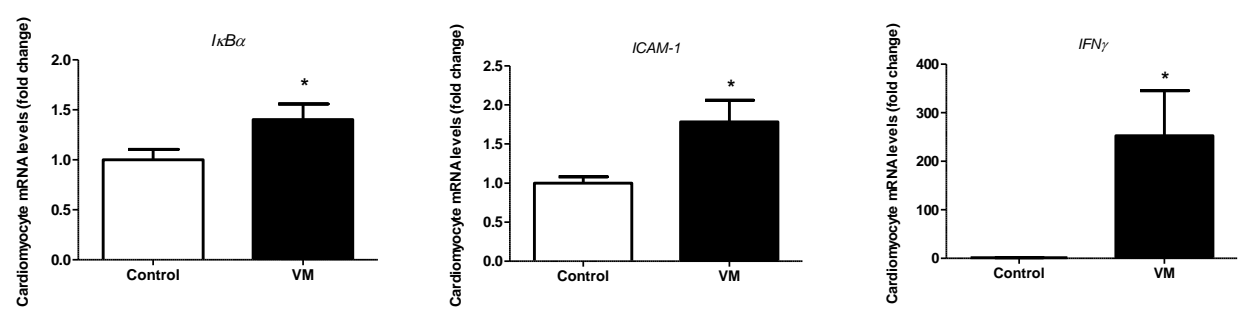

Figure S7. NF-KB activation in cardiomyocytes during CVB-3-induced VM. C3H mice were subjected to CVB3-induced $V M(n=12)$ or sham treatment $(n=5)$. QPCR analysis on intact hearts revealed increased expression of inflammatory mediators in the myocardium (A). Cardiomyocytes were isolated from mice with CVB3-induced VM ( $n=8)$ and shamtreated animals $(n=8)$ and QPCR analyses revealed increased expression levels of constituents and known target genes of the NF-KB pathway (B). ${ }^{*} p \leq 0.05$ compared to control. 
A

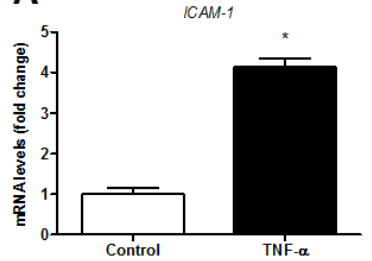

\section{HL-1 Cardiomyocytes}

Ad-GFP Ad-IKK $\beta$

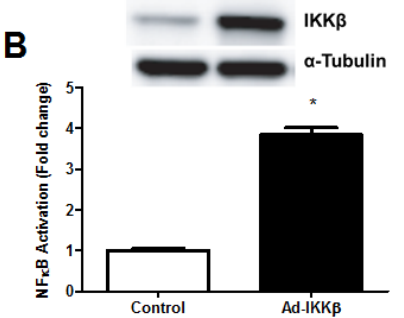

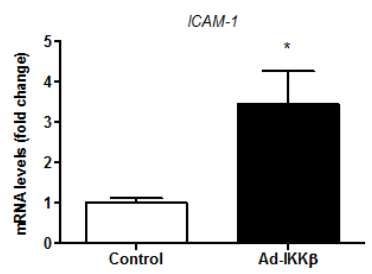

\section{Neonatal Cardiomyocytes}

C

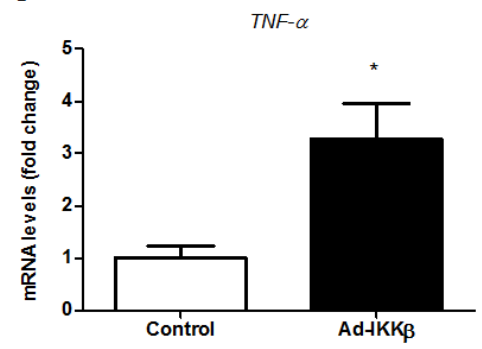

E

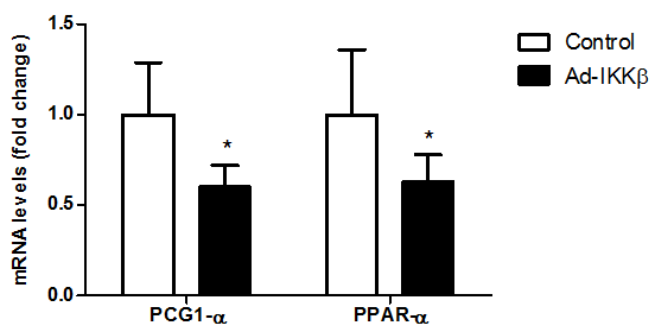

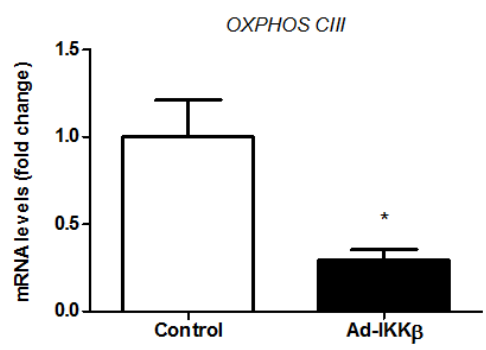

Figure S8. Activation of NF-KB signalling impairs cardiomyocyte oxidative gene expression. HL-1 cardiomyocytes were stimulated with TNF- $\alpha(10 \mathrm{ng} / \mathrm{ml})$ for $48 \mathrm{~h}$ and expression levels of ICAM-1were determined (A). HL-1 cardiomyocytes were subjected to adenoviral over-expression of IKKB or an adenoviral construct expressing GFP as a control for $48 \mathrm{~h}$. IKKB protein was increased and NF-KB transcriptional activity as well as ICAM-1 mRNA expression was induced (B). Rat neonatal cardiomyocytes were cultured and infected with an adenovirus over-expressing IKKB (Ad-IKKB) for 48h. QPCR analyses were performed and revealed increased inflammatory gene expression (C) and decreased transcript abundance of components of the oxidative phosphorylation chain (D) and constituents of PGC-1 signalling (E). Luciferase activity was corrected for total protein content. Western blots were corrected for tubulin \# $p \leq 0.1{ }^{*} p \leq 0.05$ compared to control. 


\section{Supplemental tables}

Table S1: Clinical characteristics of VM patients.

\begin{tabular}{lcc}
\hline & Controls & VM \\
\hline Age & $34.2 \pm 3.0$ & $28.3 \pm 0.33$ \\
Ejection Fraction (\%) & $56.0 \pm 3.4$ & $24.3 \pm 4.8^{*}$ \\
End Diastolic Dimension & $56.6 \pm 2.3$ & $53.7 \pm 0.3$ \\
End Systolic Dimension & $39.8 \pm 2.4$ & $47.7 \pm 1.3^{\#}$ \\
Interventricular Septum & $8.2 \pm 0.5$ & $10.3 \pm 0.9^{\#}$ \\
Wall Thickness & $8.1 \pm 0.6$ & $10.0 \pm 0.6^{\#}$ \\
PVB19 load & $77.3 \pm 36.3$ & $3105.6 \pm 140.9^{*}$ \\
CD45 $\left(/ \mathrm{mm}^{2}\right)$ & $6.2 \pm 1.1$ & $27.5 \pm 11.6^{*}$ \\
\hline
\end{tabular}

Table S2: Top 10 enriched pathways in VM patients.

\begin{tabular}{|c|c|c|c|}
\hline Pathway & Enriched & Z Score & p-value \\
\hline 1. Electron Transport Chain & $66.67 \%$ & 8,88 & 0 \\
\hline 2. Oxidative phosphorylation & $68.75 \%$ & 7,02 & 0 \\
\hline 3. Pathogenic Escherichia coli infection & $58.49 \%$ & 5,65 & 0 \\
\hline 4. Fatty Acid Beta Oxidation & $54.55 \%$ & 3,93 & 0 \\
\hline 5. TCA Cycle & $64.71 \%$ & 3,78 & 0 \\
\hline 6. Mitochondrial LC-Fatty Acid Beta-Oxidation & $66.67 \%$ & 3,73 & 0 \\
\hline 7. Fatty Acid Biosynthesis & $59.09 \%$ & 3,7 & 0 \\
\hline 8. TSH signaling pathway & $43.94 \%$ & 3,57 & 0 \\
\hline 9. Focal Adhesion & $35.33 \%$ & 3,28 & 0,003 \\
\hline 10. miR-targeted genes in squamous cell - TarBase & $36.75 \%$ & 2,96 & 0,003 \\
\hline
\end{tabular}

Table S3: Top 10 enriched pathways in CVB3-induced VM in mice.

\begin{tabular}{|c|c|c|c|}
\hline Pathway & Enrichment & Z Score & p-value \\
\hline 1. Electron Transport Chain & $91.30 \%$ & 5,51 & $<0,001$ \\
\hline 2. Oxidative phosphorylation & $97.50 \%$ & 4,98 & $<0,001$ \\
\hline 3. MAPK signaling pathway & $78.08 \%$ & 4,78 & $<0,001$ \\
\hline 4. XPodNet - protein-protein interactions in the podocyte & $66.15 \%$ & 4,5 & $<0,001$ \\
\hline 4. PodNet: protein-protein interactions in the podocyte & $70.71 \%$ & 4,27 & $<0,001$ \\
\hline 5. Insulin Signaling & $74.83 \%$ & 4,04 & 0,001 \\
\hline 6. Apoptosis & $81.08 \%$ & 3,91 & $<0,001$ \\
\hline 7. TCA Cycle & $93.33 \%$ & 3,84 & 0,001 \\
\hline 8. Chemokine signaling pathway & $73.05 \%$ & 3,78 & $<0,001$ \\
\hline 9. IL-5 Signaling Pathway & $80.88 \%$ & 3,71 & $<0,001$ \\
\hline 10. MicroRNAs in Cardiomyocyte Hypertrophy & $79.22 \%$ & 3,65 & 0,001 \\
\hline
\end{tabular}

Table S4: Correlations in gene expression levels in hearts of mice with CVB3-induced VM ( $n=12)$.

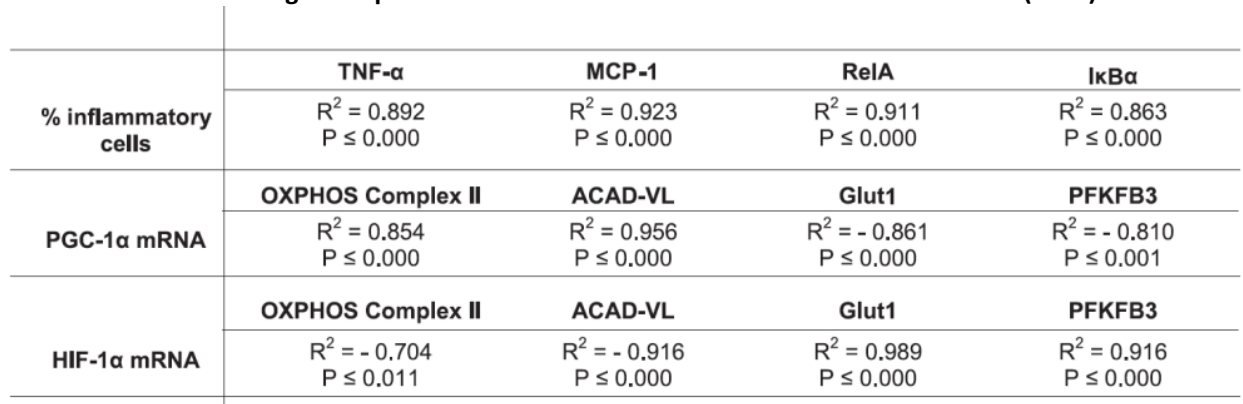




\section{Supplemental references}

1. Gautier L, Cope L, Bolstad BM and Irizarry RA. affy--analysis of Affymetrix GeneChip data at the probe level. Bioinformatics. 2004;20:307-15.

2. Du P, Kibbe WA and Lin SM. lumi: a pipeline for processing Illumina microarray. Bioinformatics. 2008;24:1547-8.

3. Ritchie ME, Phipson B, Wu D, Hu Y, Law CW, Shi W and Smyth GK. limma powers differential expression analyses for RNA-sequencing and microarray studies. Nucleic Acids Res. 2015;43:e47.

4. Kutmon M, van lersel MP, Bohler A, Kelder T, Nunes N, Pico AR and Evelo CT. PathVisio 3: an extendable pathway analysis toolbox. PLoS Comput Biol. 2015;11:e1004085.

5. Kutmon M, Riutta A, Nunes N, Hanspers K, Willighagen EL, Bohler A, Melius J, Waagmeester A, Sinha SR, Miller R, Coort SL, Cirillo E, Smeets B, Evelo CT and Pico AR. WikiPathways: capturing the full diversity of pathway knowledge. Nucleic Acids Res. 2015.

6. Papageorgiou AP, Heggermont W, Rienks M, Carai P, Langouche L, Verhesen W, De Boer RA and Heymans $\mathrm{S}$. Liver $\mathrm{X}$ receptor activation enhances CVB3 viral replication during myocarditis by stimulating lipogenesis. Cardiovasc Res. 2015;107:78-88.

7. Remels AH, Gosker HR, Bakker J, Guttridge DC, Schols AM and Langen RC. Regulation of skeletal muscle oxidative phenotype by classical NF-kappaB signalling. Biochim Biophys Acta. 2013;1832:1313-25.

8. Wu Z, Puigserver P, Andersson U, Zhang C, Adelmant G, Mootha V, Troy A, Cinti S, Lowell B, Scarpulla RC and Spiegelman BM. Mechanisms controlling mitochondrial biogenesis and respiration through the thermogenic coactivator PGC-1. Cell. 1999;98:115-24.

9. Gugneja S, Virbasius CM and Scarpulla RC. Nuclear respiratory factors 1 and 2 utilize similar glutaminecontaining clusters of hydrophobic residues to activate transcription. Mol Cell Biol. 1996;16:5708-16.

10. Gosker HR, van Mameren H, van Dijk PJ, Engelen MP, van der Vusse GJ, Wouters EF and Schols AM. Skeletal muscle fibre-type shifting and metabolic profile in patients with chronic obstructive pulmonary disease. Eur Respir J. 2002;19:617-25. 


\section{Chapter 4}

\section{MicroRNAs act as pleiotropic regulators of heart failure progression}

A high-throughput phenotypical screen in three primary cardiac cell types

\section{Authors:}

Robin Verjans $1,2 *$

Wouter Derks ${ }^{1,2 *}$

Kerstin Korn ${ }^{1}$

Birte Sönnichsen ${ }^{1}$

Rick van Leeuwen ${ }^{2}$

Blanche Schroen ${ }^{2}$

Marc van Bilsen 2,3

Stephane Heymans ${ }^{2}$

1.) Cenix BioScience $\mathrm{GmbH}$, Dresden, Germany.

2.) Department of Cardiology, Cardiovascular Research Institute Maastricht (CARIM), Maastricht University, Maastricht, the Netherlands.

3.) Department of Physiology, Cardiovascular Research Institute Maastricht (CARIM), Maastricht University, Maastricht, the Netherlands

* RV and WD Contributed equally

In preparation 


\begin{abstract}
MicroRNAs (miRNAs) have been shown to regulate pathophysiological processes underlying heart failure development, including hypertrophy and fibrosis. Recently, inflammatory miRNAs have been identified to be involved in cardiac hypertrophy and remodelling. The present study aimed to screen the relatively unknown role of a large number of inflammation-related miRNAs in cellular processes underlying cardiac failure. Based on microarray and RNA deep sequencing analyses in cardiac left ventricular samples of rodents and human, we selected 194 miRNAs that were differentially expressed in, and possibly related to, the inflammatory component of cardiomyocyte hypertrophy, fibroblast-mediated collagen production, and macrophage polarization. Following transfection with these miRNA mimics and by means of automated microscopy we quantified: (a) cell size of cardiac myocytes, (b) collagen 1 1 1-positive area in cardiac fibroblast and (c) morphological features associated with polarization status (cell roundness) and nuclear translocation of the NFkB subunit p65 in primary macrophages. Of the tested miRNAs, $13 \%$ significantly modulated cardiomyocyte size (hypertrophy) by more than $2 x$ the standard deviation of controls under unstimulated conditions. Using similar criteria, $32 \%$ of the tested miRNAs modulated collagen production by cardiac fibroblasts and $41 \%$ modulated macrophage polarization. Notably, 16 miRNAs significantly affected all three cellular processes, including miRNAs with established (miR-199-3p, miR-133b) and unknown (miR-200c-3p, miR-891a-3p) roles in cardiac pathophysiology. In conclusion, we identified roles for previously uncharacterized miRNAs in cardiac remodelling and were able to confirm known and attribute new effects to various more well-known miRNAs, thereby opening new avenues for the treatment of heart failure via miRNA-directed therapeutic approaches.
\end{abstract}




\section{Introduction}

Heart failure (HF), with its high prevalence and mortality rates ${ }^{1}$, is a huge societal burden ${ }^{2}$. The development of HF is characterized by hypertrophy of cardiac myocytes and excessive extracellular matrix deposition by fibroblasts. More recently it was recognized that HF is associated with a persistent pro-inflammatory state, characterised amongst others by infiltration of activated pro-inflammatory macrophages and polarisation of these macrophages towards a pro inflammatory (M1) phenotype ${ }^{3}$. This diverse spectrum of pathological events marks the complexity of HF development.

Specific microRNAs (miRNAs) have been shown to regulate the pathological processes underlying HF development ${ }^{4-6}$. MiRNAs represent a class of small ( \pm 22 nucleotides in length) non-coding RNA molecules that negatively regulate target gene expression 7,8 . MiRNA expression levels vary between cell types and have been shown to change under pathological conditions, including cardiac disease ${ }^{9}$. Recent studies indicate that the effect of miRNAs is not restricted to the cell type(s) in which they are expressed, as they are secreted via extracellular vesicles. In this way, miRNAs can act as paracrine signalling molecules on neighbouring cells ${ }^{10-12}$. Thus, despite various miRNAs associated with HF development being identified, our understanding of their regulating potential and the cardiac cell types in which they exert their function remains incomplete.

Thus, based on previous, published and unpublished studies from our group, we selected 194 miRNAs that were differentially expressed primarily in hearts of HF patients and in different animal models of inflammatory cardiac diseases such as viral myocarditis and acute cardiac rejection ${ }^{6,13}$. Next, we developed a high-throughput phenotypic screening platform to assess their regulatory function in both cardiac myocytes, fibroblasts and macrophages. MiRNA mimics were transfected into primary cultures of neonatal rat cardiac myocytes (nRCMs) and fibroblasts (nRCFs) to test their effects on the hypertrophic and fibrotic response, respectively. In addition, we screened these miRNAs for their influence on the polarization and activation of mouse bone marrow-derived macrophages (BMDMs). The simultaneous testing of this subset of miRNAs in different cell types under unstimulated and stimulated conditions allowed us to assess the role of these miRNAs in the different cellular processes underlying HF development in an integrative manner and to unravel pleiotropic effects of multiple miRNAs.

\section{Materials and Methods}

\section{miRNA selection}

miRNA expression profiling of patients diagnosed with viral myocarditis (VM), patients which underwent cardiac transplantation (HTX), and three cardiac disease animal models: Coxsackievirus B3 induced-VM, mouse HTX, and the ZSF1 model, resulted in the identification of 194 differentially expressed miRNAs. Several identified miRNAs showed an overlap between the different studied cardiac disease models. Both miRNA strands were included in the screen when the miRNA profiling data did not specify which miRNA strand (3$\mathrm{p} / 5-\mathrm{p})$ was measured. Supplemental Table 1 shows all miRNAs selected for in vitro validation based on a significantly altered expression in one or more cardiac disease animal models, indicating a possible regulatory role in cardiac disease progression and development. 


\section{Isolation and culture of primary cells}

Rat neonatal ventricular cardiac myocytes (nRCMs) and rat neonatal cardiac fibroblasts (nRCFs) were isolated by enzymatic dissociation from the hearts of 1-3 day old Wistar rats and cultured as described previously ${ }^{14}$. Briefly: After decapitation, neonatal rat pups were dissected using a cut through the sternum and hearts were collected under semi-sterile conditions. Subsequently, atria were removed and the ventricles were divided into 8-12 equal parts. Ventricles were consecutively digested with a mixture of $0.3 \mathrm{mg} / \mathrm{ml}$ Collagenase (Sigma \#C2674) and $0.3 \mathrm{mg} / \mathrm{ml}$ Pancreatin (Sigma \# P3292) in ADS buffer (120 mM NaCl, 5 $\mathrm{mM} \mathrm{KCl} 0.8 \mathrm{mM} \mathrm{MgSO}_{4}, 0.5 \mathrm{mM} \mathrm{KH}_{2} \mathrm{PO}_{4}, 0.3 \mathrm{mM} \mathrm{Na}_{2} \mathrm{HPO}_{4}, 20 \mathrm{mM}$ HEPES, $5.6 \mathrm{mM}$ Glucose, $\mathrm{pH} 7.35$ ) at $37^{\circ} \mathrm{C}$. Every $20 \mathrm{~min}$ the supernatant was collected, suspended in $10 \% \mathrm{NBCS}$, and $10 \mathrm{ml}$ fresh enzyme solution was added to the residual tissue. After 5 rounds of incubation, cell solutions were pooled, centrifuged, and re-suspended in nRCM plating medium (DMEM 11966, 17\% M199 medium, 10\% HS, 5\% NBCS) and pre-plated onto $162 \mathrm{~cm}$ Corning Costar cell culture flasks (Sigma \#CLS3151). After incubation in a humidified $37{ }^{\circ} \mathrm{C} / 5 \%$ CO2 incubator for $1 \mathrm{~h}$, the supernatant, containing mainly nRCMs, was collected and cells therein were counted manually. Adherent nRCFs remaining in the flask were cultured for 2 more days in nRCF medium (DMEM 22320, 10\% FBS).

Bone marrow-derived macrophages (BMDMs) were generated as previously described ${ }^{15}$, in brief: Bone marrow cells were isolated from 12 week old C57BL/6-N mice and cultured in RPMI supplemented with 15\% L929 conditioned medium (containing M-CSF) in petri dishes for 7 days to generate BMDMs. Cells were lifted for plating at day 8 using cell scrapers.

\section{Culture, transfection and stimulation of primary cell culture.}

nRCM: nRCMs were seeded into $1 \%$ gelatin coated 96 -well black, clear bottom, culture plates (Corning \#3603) at a density of 40,000 cells/well in $100 \mu$ l seeding medium. Prior to cell seeding, $0.171 \mu$ l Lipofectamine 2000 (Invitrogen \#11668-019) transfection reagent (diluted 700x in OPTIMEM) was added to the wells in combination with mirVana mimics (LifeTechnologies) according to manufacturer's protocol to end up with a final concentration of $10 \mathrm{nM}$. The day after isolation, cells were washed and medium was replaced for $100 \mu \mathrm{l}$ serumfree experimental medium (DMEM 11966, 20\% M199 medium) to starve cells for $24 \mathrm{~h}$ hours. After starvation, cells were stimulated for 72 hours with $5 \mu \mathrm{M}$ phenylephrine (PE) (Sigma \#P6126) or treated with PBS as control.

nRCF: nRCFs were lifted using trypsin/EDTA 48 hours after isolation, washed, centrifuged and seeded into uncoated 384 wells $\mu$ clear plates (Greiner \#781092) in $30 \mu \mathrm{l} \mathrm{RCF}$ medium containing $10 \%$ FBS. A seeding density of 2000 cells/well was used. After $24 \mathrm{~h}$ and prior to transfection, cells were washed extensively using an automated washer dispenser (EL406, BioTek) and $30 \mu$ low serum (0.1\% FBS) nRCF medium was added. Lipofectamine RNAiMax (Invitrogen) was mixed with OPTIMEM and mirVana mimics (Life-Technologies) according to manufacturer's protocol and $5 \mu \mathrm{l}$ of the mix was added to the wells, to give a final concentration of $20 \mathrm{nM}$ for mimics and a dilution factor of RNAiMAX of 700x. After $24 \mathrm{~h}$ of starvation, cells were stimulated with $10 \mathrm{ng} / \mathrm{ml} \mathrm{TGF \beta 1}$ (Peprotech hum. Rec TGF $\beta 1$ \# 100-21) or vehicle (PBS).

BMDM: BMDMs were lifted using a scraper after 8 days of differentiation. Subsequently, cells were counted and seeded at a density of 2000 cells/well into uncoated 384 wells $\mu$ clear plates (Greiner \#781092) in $30 \mu \mathrm{l} \mathrm{BDMD} \mathrm{medium.} \mathrm{After} \mathrm{24h,} \mathrm{medium} \mathrm{was} \mathrm{refreshed} \mathrm{prior} \mathrm{to}$ transfection. Viromer Green (Lypocalyx, Halle, Germany) was mixed with Buffer $F$ and 
mirVana mimics (Life-Technologies) according to manufacturer's protocol and $5 \mu$ of the mix was added to the wells, to give a final concentration of $20 \mathrm{nM}$ mimics and a dilution factor of 1800x for the transfection reagent. $24 \mathrm{~h}$ after transfection, cells were stimulated with 20 $\mathrm{ng} / \mathrm{ml} \mathrm{IL-4,} 20 \mathrm{ng} / \mathrm{ml} \mathrm{IFN}$ (Peprotech), $50 \mathrm{ng} / \mathrm{ml}$ LPS (Sigma) or vehicle (PBS).

\section{Immunostaining and Microscopy}

All cell types were fixed with $4 \%$ paraformaldehyde ( $45 \mathrm{~min}$ ) and treated with blocking buffer (15\% FBS, 0.3\% Triton X-100, 0.03\% Saponin in PBS) for 45 min. nRCMs were treated with permeabilisation buffer ( $3 \%$ Triton $X-100$ ) for 10 minutes preceding blocking procedure. After blocking, nRCMs were incubated for 3 hours at $37^{\circ} \mathrm{C}$ with the primary antibodies mouse anti$\alpha$-Actinin (1:1000, Sigma, \#A7811), and rat anti- $\alpha$-Tubulin (1:1500, Serotec \#MCA77G). nRCFs were treated with rabbit anti-Collagen $1 \alpha 1$ (1:500, Abcam \#AB34710) and rat anti- $\alpha$-Tubulin (1:750) antibody overnight at 4ㅇ. BMDMs were treated with rabbit NFKB p65 antibody (1:300 Santa Cruz \#sc-372) and rat anti- $\alpha$-tubulin (1:750) overnight at 4 으. After primary antibody incubation, nRCMs and nRCFs were incubated with secondary antibodies goat antirat AlexaFluor555 (1:750, Life Technologies, \#A11006), goat anti-mouse AlexaFluor488 (1:500, Life Technologies, \#A11001) and Hoechst (1:6000, Invitrogen \#H3570) for 1 hour at room temperature. BMDMs were incubated with secondary antibodies goat anti-rat AlexaFluor555 (1:500), goat anti-rabbit AlexaFluor488 (1:750, Life Technologies, \#A11008) and Hoechst for 1 hour at room temperature.

\section{Acquisition \& image analysis}

For the screening experiments, image acquisition was performed using an ImageXpress Micro automated high-content screening fluorescence microscope (Molecular Devices) at $\times 10$ magnification with FITC, CY3 and DAPI filter blocks. For the hypertrophy, fibrosis, and inflammation screen, a total of respectively 25, 16, and 9 images were acquired per wavelength per well.

Image analysis was performed using the eCognition software (Definiens, platform (Munich, Germany). Number of nuclei was determined based on Hoechst staining for all three cell types. $\mathrm{nRCM}$ and nRCF cell size was quantified using an algorithm that recognized $\alpha$-tubulin stained area. For nRCFs, collagen $1 \alpha 1$ positive area was quantified as a percentage of cell area. For BMDMs, cell roundness was determined as a percentage of cells detected with a length-width ratio larger then threshold. NFkB nuclear translocation was based on p65 positive area in nucleus (Hoechst) and cytoplasm ( $\alpha$-tubulin stained area) and expressed as ratio of nuclear/cytoplasmic p65 staining.

\section{Data analysis and statistics}

For all analysed miRNA samples, mean and standard deviation of individual wells over 3 replicate plates was determined. Inter-plate variability was corrected by scaling each data point to the corresponding negative control mean (negative control mimic transfected cells). The effect of miRNA transfection on different read outs was determined via calculation of the $\log _{2}$ fold change of the sample mean over the negative control mean within the same condition and statistically tested using an unpaired T-Test.

In all three screens, identification of miRNA mimic-induced phenotypical changes was based on a statistically significant $(p<0.05)$ sample mean $\log _{2}$ fold change of the main read out over negative control, deviating more than $2 x$ the STDEV from the negative control mean. The main readouts per screen were cell size for the hypertrophy screen, number of 
nuclei and collagen content for the fibrosis screen, and cell roundness and NFKB nuclear translocation for the inflammation screen. Only hit selection for the NFKB nuclear translocation read out in the inflammation screen was based on statistically significant mean $\log _{2}$ fold change over negative control, deviating more than $1 x$ the STDEV from the negative control mean. 


\section{Results}

We assembled a library of 194 miRNA mimics based on their differential expression in the hearts of different animal models of inflammatory cardiac disease, patients diagnosed with viral myocarditis, and patients undergoing cardiac transplantation (Supplemental table 1). To screen for involvement of these miRNAs in cellular processes driving HF, this HF-associated miRNA mimic library was overexpressed in three primary cell types held accountable for pathological cardiac remodelling (Figure 1).

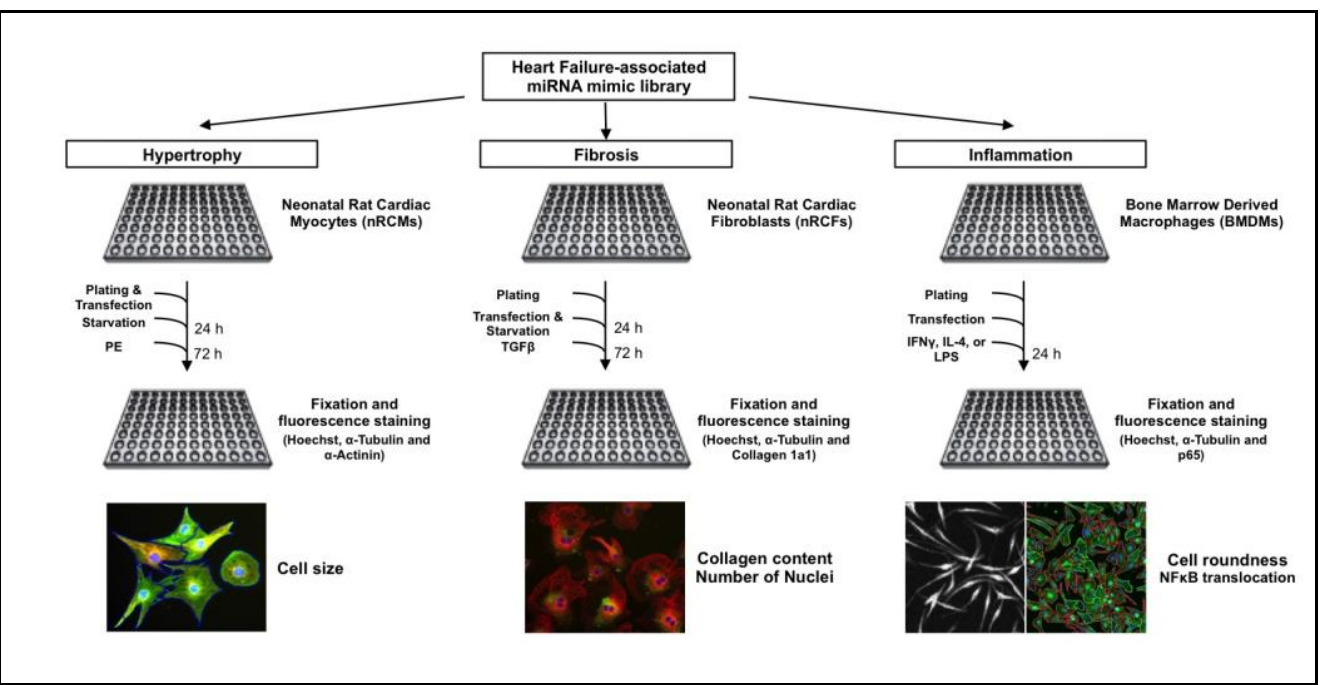

Figure 1. Phenotypical screening using a HF-associated miRNA mimic library was performed in parallel for three different HF-underlying processes using automated image analysis. Effects induced under control and HF-mimicking conditions by miRNA mimics on hypertrophy, fibrosis and inflammation were studied using nRCMs, nRCFs, and $B M D M s$ respectively. Read-outs were cardiac myocyte size, cardiac fibroblast number and collagen content, and macrophage roundness and NFKB translocation.

\section{Hypertrophy screen}

MiRNA mimics were transfected into unstimulated and PE-stimulated neonatal rat cardiac myocytes (nRCMs) and cell size was quantified to determine their effect on the hypertrophic response. PE stimulation led to cardiomyocyte hypertrophy, characterized by a $0.30 \log _{2}$ fold increase in cell size and increased transcript levels of hypertrophic markers ANF and BNP (Supplementary figure 1). Functional effects induced by transfection of miRNA mimics in nRCMs was first verified using the established anti-hypertrophic miRNA miR-1 ${ }^{4}$. Transfection with a miR-1 mimic resulted in a significant reduction of cell size, a $42 \%$ reduction in mRNA expression level of direct target CALM2, and prevented the PE-induced upregulation in transcript levels of ANF and BNP (Supplementary figure 1).

The effect of each miRNA on nRCM cell size under unstimulated conditions, relative to negative control-mimic transfected cells (further referred to as negative control), is presented in a vulcano plot (Figure 2A). Three miRNAs, miRNA-200c-3p, miR-125a-5p, and miR-92b-3p, significantly increased cell size with a $\log _{2}$ fold change greater than $2 x$ STDEV of negative control, and 22 miRNAs were found to reduce cell size with a similar fold change 
(Figure 2A). Among the latter, miR-486-3p, miR-145-3p, and our positive control miR-1 reduced cell size significantly.

Control

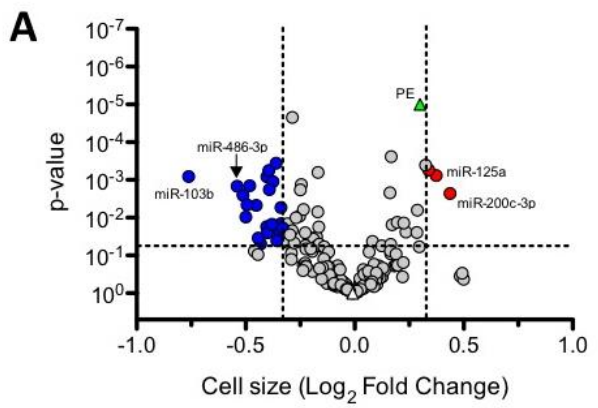

PE

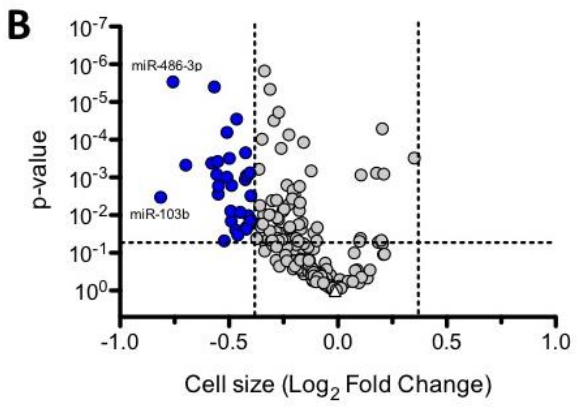

C

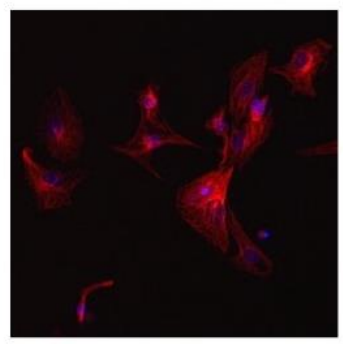

miR-486-3p

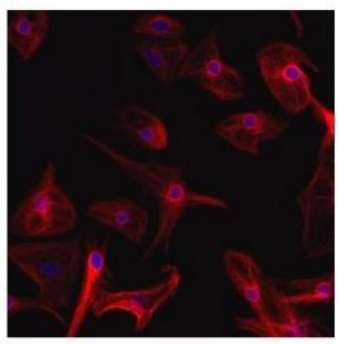

Negative Control

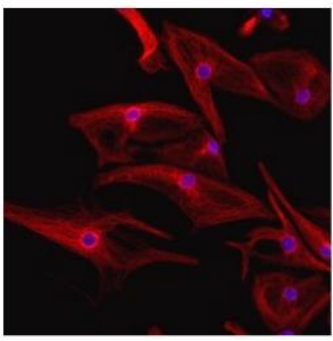

miR-125a-5p

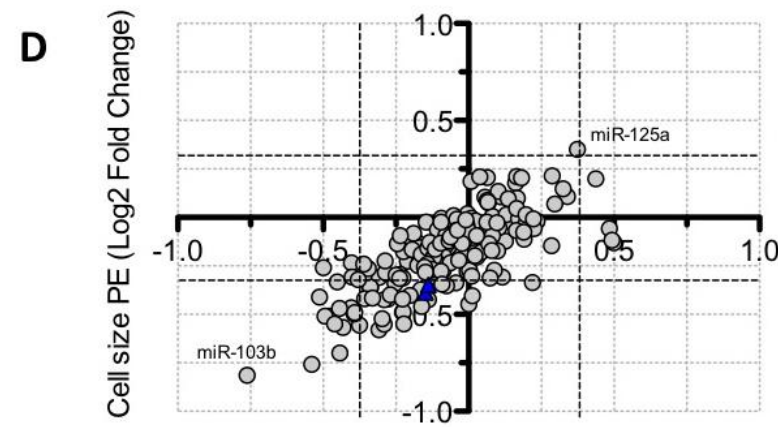

\section{Cell size Control ( $\log _{2}$ Fold Change)}

Figure 2. Hypertrophy screen identifies miRNAs that affect cardiac myocyte cell size. Panel $A-B$ shows $\log _{2}$ fold changes in cell size compared to negative control without (panel A) or with (panel B) PE stimulation. MiRNA mimics that induce a significant effect on cell size, deviating more than $2 x$ STDEV from negative control (indicated by vertical lines) are highlighted in red or blue. The PE-induced increase in cell size after $72 \mathrm{~h}$ is represented in green in panel $A$. Panel $C$ shows representative images of the miRNAs with most pronounced effect on cells size compared to negative control, miR-103b and miR-125a-5p. Panel D shows the effect of individual miRNA mimics on cardiac myocyte cell size under both unstimulated (control) and stimulated (PE) conditions. Transfection of mimics for miR-151-3p and $-5 p$ reduced cell size under PE-stimulated conditions without having an effect under control conditions (shown in blue). Values represent cardiomyocyte cell size expressed as $\log _{2}$ fold change, normalised to negative control mimictransfected cells within the same condition. 
PE stimulation triggered the hypertrophic response in $\mathrm{nRCMs}$, inducing a $0.30 \log _{2}$ fold increase in cardiac myocyte cell size (Figure 2A). Under PE-stimulated conditions, 8 miRNAs induced an additional significant increase in cell size compared to PE-stimulated negative control. However, none of these miRNAs induced an increase in cell size deviating more than 2x STDEV of negative control (Figure 2B). In contrast, 31 miRNAs were found to significantly reduce the $\mathrm{PE}$-induced increase in cardiac myocyte cell size by more than $2 x$ STDEV of negative control, among which miR-103b, miR-486-3p, miR-151a-3p, miR-145-3p, and miR891a-3p (Figure 2B). The top 5 miRNAs identified to increase or decrease cardiac myocyte cell size under control conditions are depicted in table 1. Effects induced after miRNA mimic transfection on cell size are shown for both control and PE-stimulated conditions. Combining the effect of the individual miRNA mimics on cardiac myocyte cell size under both unstimulated (control) and stimulated (PE) conditions (Figure 2D) reveals that miR-125a-5p and miR-103b have the most pronounced effect on cell size independent of PE stimulation. Interestingly, both miR-151a-3p and miR-151a-5p decreased cardiac myocyte cell size only in combination with PE-treatment (Figure 2D, shown in blue).

Table 1. Top 5 miRNAs able to increase or decrease cardiac myocyte cell size.

\begin{tabular}{lcccc}
\hline \multirow{2}{*}{ miRNA } & \multicolumn{2}{c}{ Control } & \multicolumn{2}{c}{ PE-stimulated } \\
& Cell size $\left(\log _{2} \mathrm{Fc}\right)$ & p-value & Cell size $\left(\log _{2} \mathrm{Fc}\right)$ & p-value \\
\hline miR-200c-3p & 0,437 & 0,002 & 0,200 & 0,047 \\
miR-125a-5p & 0,374 & 0,001 & 0,351 & 0,000 \\
miR-92b-3p & 0,339 & 0,001 & 0,108 & 0,441 \\
\hline miR-103b & $-0,761$ & 0,001 & $-0,814$ & 0,003 \\
miR-486-3p & $-0,540$ & 0,001 & $-0,757$ & 0,000 \\
miR-103a-3p & $-0,513$ & 0,003 & $-0,411$ & 0,010 \\
miR-1 & $-0,500$ & 0,010 & $-0,260$ & 0,058 \\
miR-214-3p & $-0,494$ & 0,005 & $-0,509$ & 0,001 \\
\hline
\end{tabular}

Table shows the $p$-value and $\log _{2}$ fold change in cardiac myocyte cell size under control and PE-stimulated conditions. Only three miRNA mimics increased cell size with more than 2x STDEV of negative control.

\section{Fibrosis screen}

To determine the effect of the HF-associated miRNAs on the fibrotic response, collagen production and proliferation of neonatal rat cardiac fibroblasts (nRCFs) were quantified. Transfection of nRCFs with a reported anti-fibrotic control mimic, miR-29b ${ }^{5}$, resulted in a decrease of $93 \%$ in transcript levels of direct target collagen-1 $\alpha 1$ (COL1 $\alpha 1$ ) (Supplementary figure $2 \mathrm{~A}$ ), confirming the functional effects of introducing the miR-29 mimic. To initiate a pro-fibrotic response cardiac fibroblasts were stimulated with TGF $\beta$. This resulted in a significant upregulation of transcript levels of the fibrosis marker connective tissue growth factor (CTGF) (Supplemental figure 2B), accompanied by an increase in collagen positive area (Figure $3 \mathrm{~A}$ ). Figure $3 \mathrm{~A}$ shows the $\log _{2}$ fold change in COL1 $\alpha 1$ positive stained cell area (further referred to as collagen content) for each mimic relative to negative control. A large number (51) of miRNA mimics significantly increased the collagen content, including miR-379-3p, miR-151a-5p, and miR-30d-5p. Twelve miRNA mimics decreased collagen content by more than 2x STDEV of negative control, among them are miR-199a-5p (-2.045 p=0.001), miR$103 a-2-5 p(-1.647 p=0.021)$ and several members of the miR-29 family: miR-29a-3p $(-2.238$ 
$p=0.009)$, miR-29b-3p $(-2.381 p=0.000)$, and miR-29c-3p $(-2.163 p=0.000)$ that are known to target several collagen subtypes ${ }^{5}$, including COL $1 \alpha 1$.
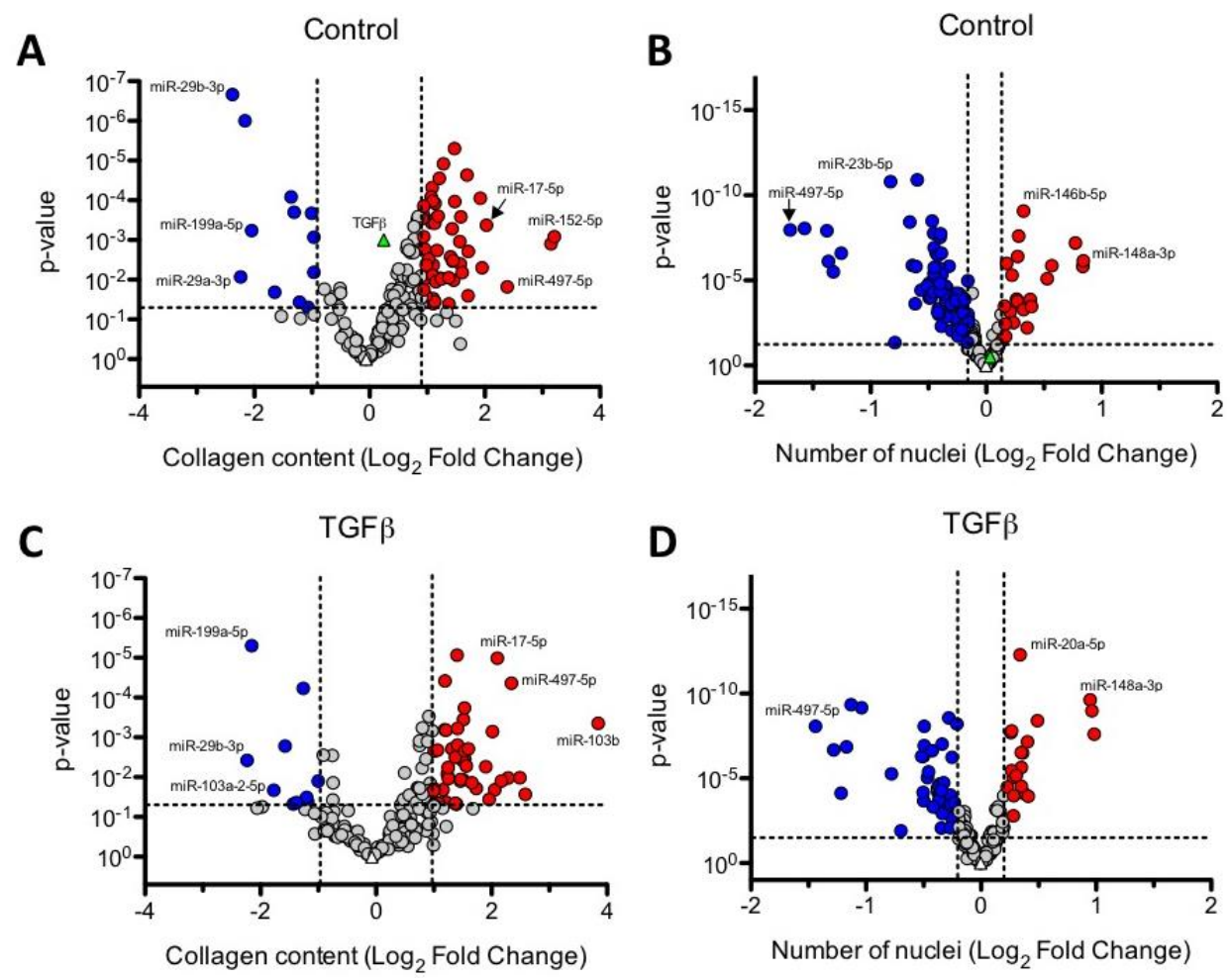

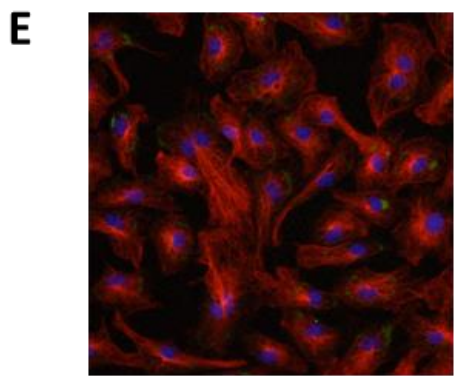

miR-199a-5p

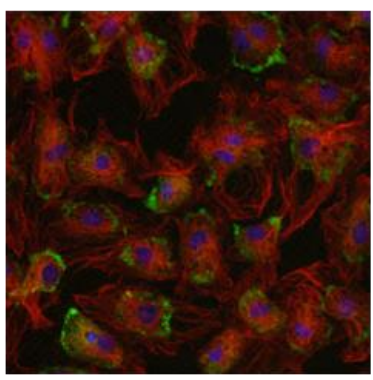

Negative control

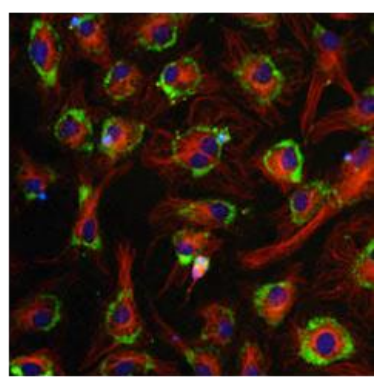

miR-17-5p

Figure 3. Effect of miRNA mimics on cardiac fibroblast collagen content and number of nuclei upon transfection. Vulcano plots showing collagen content and number of nuclei for individual miRNA mimics without (panel $A$ and B) or with (panel $C$ and D) TGFB stimulation for 72 hours, expressed as $\log _{2}$ fold change compared to negative control mimic-transfected nRCFs. MiRNA mimics that increase or reduce collagen content are highlighted in red or blue, respectively. As a point of reference, the effects that are reached by 72 hours of TGFB stimulation of untransfected controls are represented as green triangles in both panels $A$ and $B$. Panel $E$ shows representative images of miR-199a and $\operatorname{miR}-379-3 p$.

Figure $3 B$ shows the effect of each individual miRNA mimic on the number of nuclei of cardiac fibroblasts. A large fraction of the tested miRNA mimics was able to significantly increase (22) or decrease (95) the number of nuclei with more than 2x STDEV. Interestingly, 
41 miRNA mimics were able to alter both cardiac fibroblast collagen content and number of nuclei under baseline conditions. Among these are members of the miR-29 family known for their anti-fibrotic effects ${ }^{5,16}$.

Table 2. Top 5 miRNAs able to increase or decrease collagen content.

\begin{tabular}{|c|c|c|c|c|}
\hline \multirow{2}{*}{ miRNA } & \multicolumn{2}{|l|}{ Control } & \multicolumn{2}{|l|}{ TGF $\beta$-stimulated } \\
\hline & Collagen content ( $\log _{2}$ f.c.) & p-value & Collagen content ( $\log _{2}$ f.c.) & p-value \\
\hline miR-152-5p & 3,141 & 0,000 & 2,513 & 0,000 \\
\hline $\operatorname{miR}-7-5 p$ & 3,086 & 0,001 & 2,419 & 0,001 \\
\hline $\operatorname{miR}-497-5 p$ & 2,325 & 0,000 & 2,273 & 0,000 \\
\hline miR-17-5p & 1,966 & 0,001 & 2,029 & 0,001 \\
\hline miR-1 & 1,889 & 0,001 & 2,217 & 0,001 \\
\hline $\operatorname{miR}-29 b-3 p$ & $-2,381$ & 0,000 & $-2,232$ & 0,004 \\
\hline miR-199a-5p & $-2,110$ & 0,000 & $-2,224$ & 0,087 \\
\hline miR-103a-2-5p & $-1,711$ & 0,000 & $-1,843$ & 0,001 \\
\hline miR-216a-5p & $-1,598$ & 0,000 & $-0,330$ & 0,459 \\
\hline miR-891a-3p & $-1,426$ & 0,000 & $-0,983$ & 0,001 \\
\hline
\end{tabular}

$\log _{2}$ fold change in collagen content under control and TGFB-stimulated conditions and p-values are shown.

Under TGF $\beta$-stimulated conditions, our screen revealed 47 and 10 miRNAs to significantly increase or decrease collagen content, respectively (Figure $3 C$ ). In the presence of TGF $\beta, 20$ miRNA mimics increased and 45 decreased the number of nuclei by more or less than $2 x$ STDEV (Figure 3D). The same as under unstimulated conditions, miR101-3p significantly decreased the number of nuclei in nRCFs with more than 2x STDEV in the presence of TGF $\beta$. Mir-101 was shown to inhibit post-infarct cardiac fibrosis by decreasing the proliferation of fibroblasts via targeting Fos ${ }^{17}$. Table 2 lists the top 5 miRNAs with the most pronounced effect on cardiac fibroblast collagen content in both directions. For each miRNA, their effect on collagen content and on the number of nuclei is shown under control and TGF $\beta$ stimulated conditions.

\section{Macrophage polarisation and activation screen in BMDMs}

Lastly, we evaluated the effect of the miRNA mimics on macrophage polarisation and activation. Transfection of BMDMs with GAPDH siRNA resulted in a decrease of $93 \%$ in GAPDH transcripts levels (Supplementary figure $3 A$ ), confirming successful transfection of BMDMs. The effect of individual mimics on macrophage polarisation was quantified under unstimulated (M0), IFN $\gamma$ (M1) and IL-4 (M2) stimulated conditions. Morphological appearance of macrophages has been shown to be a marker of polarization status in BMDMs 18 , with M1 (pro-inflammatory) macrophages showing a round and M2 (anti-inflammatory) polarized macrophages showing an elongated phenotype. Stimulation of BMDMs with IFN $\gamma$ resulted in a $27 \%$ increase in the number of round classified cells and IL-4 stimulation resulted in a $21 \%$ increase in elongated classified cells (Figure 4A). In unstimulated macrophages, four miRNA mimics significantly decreased macrophage roundness with more than $2 x$ STDEV, these are: miR-488-5p, miR-34a-3p, miR-891a-3p, and miR-128-3p. A large number (76) of miRNAs significantly increased roundness (Figure 4A), among them miR-200c$5 p$, miR-148b-3p, and miR-155-5p, which is known to induce M1 polarisation ${ }^{19}$. Comparable 
results were found for macrophages polarised towards M1 (IFNY) and M2 (IL-4) (Supplemental figure 3B-C). In total, 58 miRNAs were able to significantly alter macrophage roundness in all three polarization conditions. Remarkably, only 2 miRNAs were identified to promote macrophage elongation irrespectively of the polarization stimuli, while 56 miRNAs increased macrophage roundness. MiR-155, a well-known pro-inflammatory miRNA, showed a pronounced and significant increase in roundness in all conditions. The top 5 miRNAs able to promote or decrease macrophage roundness, as identified under control conditions, along with their effect on NFkB nuclear translocation, are shown in Table 3.

A

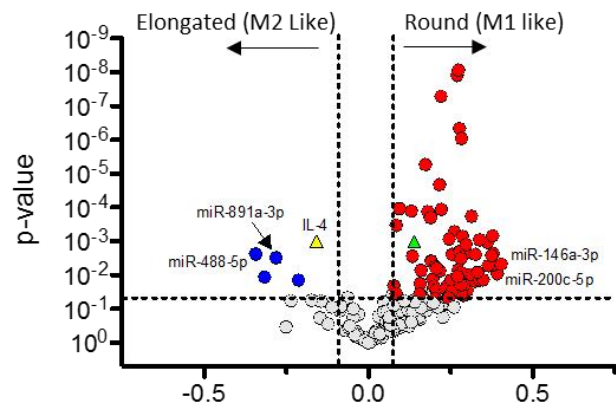

Roundness ( $\log _{2}$ Fold Change)
B

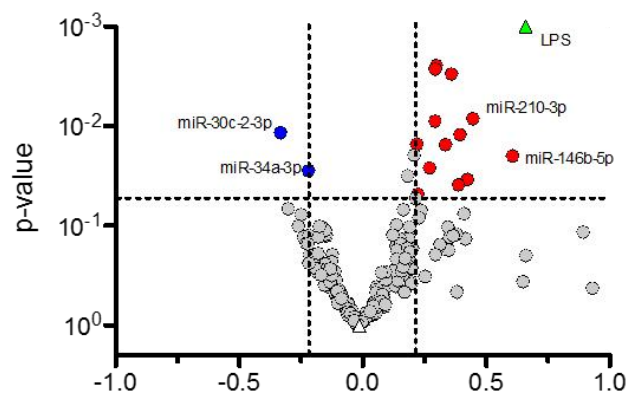

$\mathrm{NFkB}$ nuclear translocation ( $\log _{2}$ Fold Change)

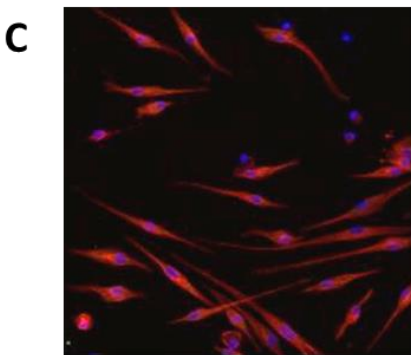

miR-488-5p

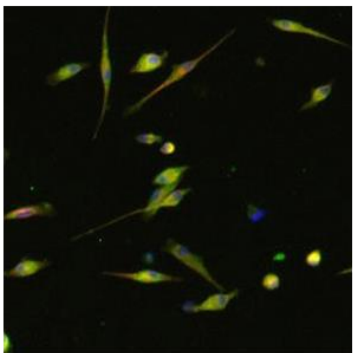

Negative control

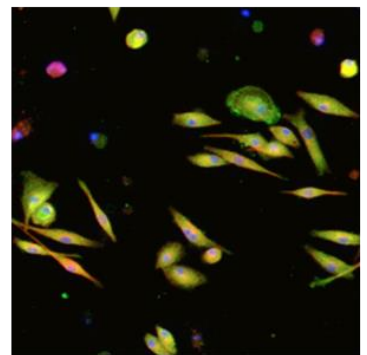

miR-146a-3p

Figure 4. Inflammation screen identifies many miRNAs affecting macrophage roundness and NFkB nuclear translocation. Vulcano plots showing $\log _{2}$ fold change in cell roundness and NFKB nuclear translocation in control conditions. MiRNA mimics that significantly increased or reduced these readouts according to previously described criteria are highlighted in red or blue, respectively. In panel A, polarization effects on cell roundness in negative control mimic-transfected cells induced by IFNY and IL-4 stimulation are represented as yellow and green triangles respectively. In panel B, LPS-induced effect on NFKB nuclear translocation in negative control mimic-transfected cells is represented as a green triangle. Panel $C$ shows representative images of miR-488-5p and miR-146a-3p with pronounced effects on macrophage roundness. 
Table 3. Top 5 miRNAs able to increase or decrease macrophage roundness.

\begin{tabular}{lcccc}
\hline \multirow{2}{*}{ miRNA } & \multicolumn{4}{c}{ Control } \\
& Roundness (Log $\mathbf{f}$.c.) & p-value & NFkB (Log $\mathbf{f}$.c.) & p-value \\
\hline miR-146a-3p & 0,404 & 0,005 & 0,171 & 0,212 \\
miR-200c-5p & 0,392 & 0,009 & 0,154 & 0,152 \\
miR-30a-3p & 0,380 & 0,002 & 0,893 & 0,116 \\
miR-148b-3p & 0,378 & 0,005 & 0,215 & 0,053 \\
miR-218-5p & 0,378 & 0,001 & 0,379 & 0,121 \\
\hline miR-488-5p & $-0,343$ & 0,002 & $-0,179$ & 0,130 \\
miR-34a-3p & $-0,317$ & 0,011 & $-0,22$ & 0,028 \\
miR-891a-3p & $-0,281$ & 0,003 & 0,05 & 0,669 \\
miR-128-3p & $-0,214$ & 0,014 & $-0,174$ & 0,101 \\
\hline
\end{tabular}

The table shows the $p$-value and $\log _{2}$ fold change in macrophage roundness and NFKB nuclear translocation under control conditions. Only four miRNA mimics reduced roundness with more than 2x STDEV of negative control.

The effect of individual mimics on the activation of BMDMs was quantified using an NFKB nuclear translocation assay either stimulated with- or in absence of LPS. Activation of negative control mimic-transfected BMDMs with LPS resulted in a significant $0.66 \log _{2}$ fold increase in NFKB nuclear translocation (Figure 4B). Only miR-30c-2-3p and miR-34a-3p were identified to significantly decrease NFKB nuclear translocation with more than $1 \mathrm{x}$ STDEV, while 13 miRNAs were found to significantly increase NFKB nuclear translocation by more than 1x STDEV $\log _{2}$ fold, including miR-210-3p, miR-590-5p, miR-96-5p, and miR-152-3p. MiR$146 b-5 p$, a positive regulator of the NFKB pathway ${ }^{20}$, induced the strongest increase in NFKB nuclear translocation. Supplemental figure 3E shows the $\log _{2}$ fold change in NFKB nuclear translocation for each individual miRNA mimic in macrophages activated with LPS.

\section{Pleiotropic effects of HF-associated miRNAs}

The vast majority of miRNAs (86\%) affected at least one of the HF-related outcome parameters (Figure $5 \mathrm{~A}$ ). Of the total miRNA-library, $27 \%$ of the selected miRNAs significantly altered cardiac myocyte size, more than half (56\%) of the tested miRNA mimics were able to significantly affect cardiac fibroblast collagen content, and $43 \%$ was identified as modulators of macrophage roundness. The overlap between the different processes is substantial, marking the relatively high number of miRNAs (32\%) affecting more than one process. Notably, 16 miRNAs influenced each of the HF-underlying processes to a significant extent (Table 4). Several interesting miRNAs are highlighted in figure 5B, visualizing their effects on all main readouts, illustrating the direction and magnitude of change for each of the studied processes. For example, miR-200c-3p was able to significantly increase cardiac myocyte cell size ( $0.44 \log _{2}$ fold change), fibroblast collagen content (0.88 $\log _{2}$ fold change), and macrophage roundness ( $0.375 \log _{2}$ fold change). The effects of each individual miRNA mimic in all three screens are shown in supplemental table 1. 
Table 4. MiRNAs affecting cardiac myocyte cell size, fibroblast collagen content, and macrophage elongation in parallel.

\begin{tabular}{lccc}
\hline \multirow{2}{*}{ miRNA } & Hypertrophy screen & Fibrosis screen & Inflammation screen \\
\cline { 2 - 4 } let-7c-3p & Cell size (Log $\mathbf{f . c .})$ & Collagen content (Log f.c.) & Roundness ( $\log _{2}$ f.c.) \\
miR-103b & $-0,253$ & $-1,069$ & 0,338 \\
miR-133b & $-0,761$ & 0,586 & 0,310 \\
miR-145-3p & $-0,403$ & 0,819 & 0,191 \\
miR-152-5p & $-0,392$ & 1,025 & 0,283 \\
miR-181b-5p & 0,235 & 3,206 & 0,291 \\
miR-199b-5p & $-0,269$ & 0,975 & 0,278 \\
miR-200c-3p & $-0,235$ & $-1,310$ & 0,239 \\
miR-210-3p & 0,437 & 0,940 & 0,375 \\
miR-212-5p & 0,285 & 0,646 & 0,273 \\
miR-29a-3p & $-0,375$ & 0,849 & 0,277 \\
miR-34a-3p & 0,195 & $-2,238$ & 0,085 \\
miR-497-5p & $-0,228$ & 0,773 & $-0,317$ \\
miR-499a-5p & $-0,443$ & 2,390 & 0,077 \\
miR-891a-3p & $-0,175$ & $-0,651$ & 0,363 \\
miR-9-5p & $-0,482$ & $-1,361$ & $-0,281$ \\
\hline Data are expressed as & $-0,404$ & 1,406 & 0,250 \\
\hline
\end{tabular}

Data are expressed as average $\log _{2}$ fold change in cardiac myocyte cell size, cardiac fibroblast collagen content, and macrophage roundness under non-stimulated control conditions. All 16 miRNA affected the $\log _{2}$ fold change of these readouts significantly with more than $2 x$ STDEV of negative control. 


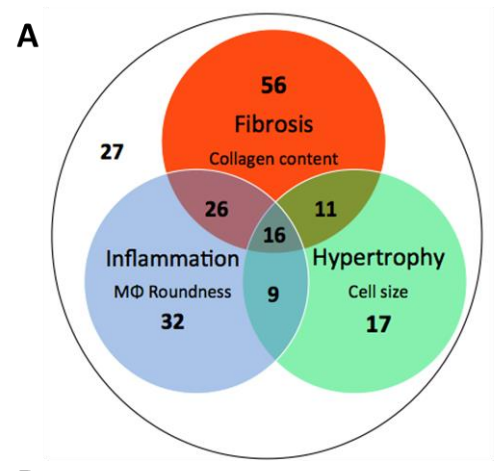

B

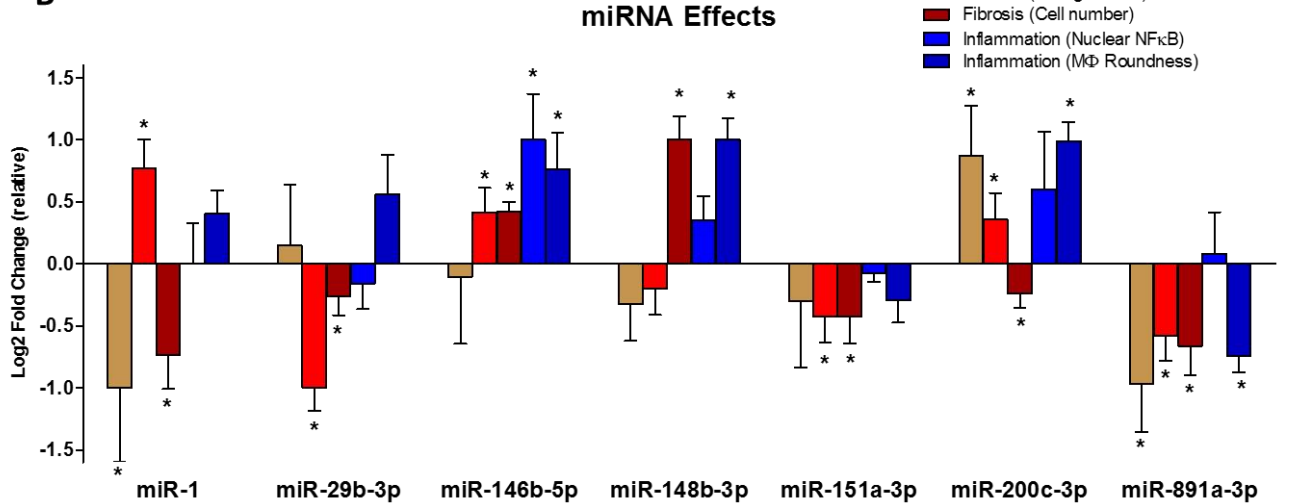

Figure 5. miRNAs affect multiple HF-related processes. A. The majority (86\%) of the miRNAs mimics affect at least one pathological process. Included read outs are cell size, collagen content, and cell roundness, for the hypertrophy, fibrosis, and inflammation screen, respectively. B. Panel B visualises the effect of a selection of miRNA mimics based on their ability to affect all main read outs or based on the usage as known HF-regulating control miRNA (miR-1 and miR-29b-3p). Bar graphs are made relative to the most pronounced miRNA-induced effect per readout. Bars are shown as Mean with SD of relative $\log _{2}$ fold change $* P<0.05$ compared to negative control. 


\section{Discussion}

In the present study, high-throughput phenotypical screening in three different primary cell types was applied to explore the effect of nearly $200 \mathrm{HF}$-associated miRNAs on three pivotal processes underlying HF development, namely hypertrophy, fibrosis, and inflammation ${ }^{1}$. Novel functions of well-studied miRNAs and less well-known miRNAs putatively involved in HF progression were identified. This comprehensive screening approach unveils the concerted actions of various miRNAs in cardiovascular disease and opens new avenues for the treatment of $\mathrm{HF}$ via miRNA-directed therapeutic approaches.

Previously phenotypical screens of miRNAs focussed on a single process, either on myocyte hypertrophy ${ }^{21}$, inflammation ${ }^{22}$, or cell proliferation ${ }^{23}$. These studies often made use of immortalised cell lines, like HL-1 cardiomyocytes and RAW 264.7 macrophages, that have lost some of their cell-type specific characteristics ${ }^{24,25}$. Furthermore, most studies only screened under basal conditions, not including stimuli that mimicked pathological processes 26. By simultaneously screening three primary cell types under basal conditions as well as after stimulation with relevant pathogenic factors, the current approach allows a better understanding of the role of these miRNAs in cardiac pathophysiology.

\section{MiRNAs that regulate cardiomyocyte hypertrophy}

We identified various miRNAs which yet have not been described to be involved in cardiac myocyte hypertrophy, and confirmed known anti- or pro-hypertrophic effects of miRNAs, such as miR-1 and miR-19a-3p (Figure 2, Table 1). By targeting calmodulin, miR-1 was shown to attenuate cardiomyocyte hypertrophy through modulation of calcium signalling ${ }^{4}$. MiR19a, on the other hand, has been shown to promote cardiac myocyte hypertrophy via targeting the anti-hypertrophic gene atrogin-1. Moreover, miR-19a plasma levels showed a positive correlation with plasma BNP and CAMP levels in HF patients ${ }^{27,28}$. Identification of these well-characterized miRNAs serves as an internal validation of our screening approach.

Transfection of 25 miRNAs mimics into cardiac myocytes altered cell size under unstimulated conditions. MiR-125a-5p, miR-200c-3p, and miR-92b-3p increased cell size, suggesting a pro-hypertrophic action. Cardiac miR-125a-5p is significantly upregulated in hypertrophic and dilated cardiomyopathy patients ${ }^{29}$, and miR-92b levels are increased in sera of patients suffering from HF and coronary artery disease ${ }^{30,31}$. Although these findings are suggestive for a role of these miRNAs is cardiac disease, prove for their actual involvement in the regulation of the hypertrophic response is provided in this study. Under PE-stimulated conditions, giving a $24 \%$ increase in cell size, 31 miRNAs mimics significantly reduced cell size, suggesting anti-hypertrophic properties. In contrast, none of the miRNAs induced an additional significant increase in cardiomyocyte size in excess of our predefined strict selection criteria of 2 times the standard deviation. Transfection with 13 miRNAs (including miR-148a-3p, miR-145-3p, miR-891a-3p, and miR-486-3p) significantly decreased cell size under both control and PE-stimulated conditions. MiR-486-3p had the most outspoken anti-hypertrophic effect, decreasing cardiomyocyte size by -0.54 and $-0.76 \log _{2}$ fold under unstimulated and PE-stimulated conditions, respectively.

Interestingly, several miRNAs seemed to have a unique PE-dependent effect. Transfection of miRNA mimics for both miR-151a-3p and $-5 p$ did not change cardiomyocyte cell size under unstimulated conditions, but decreased cell size significantly following PEtreatment. This suggests that miR-151a inhibits the hypertrophic response by targeting genes involved in the alpha-adrenergic signalling pathway. A previously performed screen ${ }^{21}$ 
identified miR-126 as anti-hypertrophic under basal conditions, while having no effect on cardiac myocyte cell size following PE treatment. In contrast, our screening approach identified miR-126-3p as anti-hypertrophic under both basal and PE-stimulated conditions. It is feasible that these differences are related to the higher dose (50 $\mu \mathrm{M})$ and/or shorter duration (48h) of PE treatment used by Jentzsch and co-workers.

\section{MiRNA mimics affecting cardiac fibroblast collagen content and proliferation}

Cardiac fibrosis is a well-established predictor of disease progression in $\mathrm{HF}^{32}$. Cardiac fibroblasts (CFs) proliferate, differentiate towards myofibroblasts, and increase their collagen production during HF disease progression ${ }^{33}$. Cellular proliferation is a commonly used readout for high-throughput screens ${ }^{23}$, mostly in cancer-related cell types, but also in fibroblasts cell lines ${ }^{34}$. As cell numbers are equal at the start, nuclei count after $72 \mathrm{~h}$ of incubation can be considered a surrogate readout for proliferation and apoptosis. Transfection with 22 miRNA mimics increased the number of nuclei. Amongst them are miR148a-3p, miR-152-3p, miR-19a-3p, and miR-195-3p, suggesting these miRNAs increase the number of fibroblasts in the heart and thereby promote cardiac fibrosis.

Conversely 95 miRNA mimics, including miR-152-5p, miR-15a-5p, miR-16-5p, and miR-195-5p, reduced the number of nuclei, pointing to an anti-proliferative or pro-apoptotic effect of these miRNA's. In this way these miRNAs attenuate the development of cardiac fibrosis. Among the miRNAs with the most outspoken anti-proliferative effect are the members of the miR-148/152 family that were previously described as tumour suppressors 35. MiR-16 which strongly reduced the number of fibroblasts in this screen, was shown to inhibit proliferation of 3 T3 fibroblasts in an earlier study ${ }^{36}$.

To determine collagen production, we quantified the relative cell area positive for collagen $1 \alpha 1$ staining. Various miRNAs showed a pronounced increase in this readout (for instance miR-152-5p and miR-497-5p, miR-152-5p, miR-7-5p, miR-17-5p, miR-1). However, for some miRNAs (miR-152-5p and miR-497-5p) this was accompanied by a significant reduction in cell number, which may confound interpretation of their effect on fibrosis (see limitations of the study). MiRNA mimics that increased collagen content without significantly effecting cell number, are miR-17-5p, miR-181c-5p, and several miR-30 family members. In line, miR-30 was shown to downregulate CTGF, a key pro-fibrotic factor ${ }^{37}$. MiRNA mimics that reduced collagen content include miR-21-3p, known for its role in cardiac fibrosis ${ }^{38}$, all members of the miR-29 family known to target collagen production ${ }^{5}$, and miR-24-1-5p, which was shown to reduce cardiac fibrosis after $\mathrm{MI}^{39}$.

\section{MiRNA mimics regulating BMDM polarisation and activation}

Here we present a novel approach for screening macrophage polarisation based on morphological features of BMDMs. Depending on their polarisation status, macrophages exhibit differences in morphology, cytoskeletal organization, functional characteristics, and migrating capacity ${ }^{40}$. We have screened for phenotypical changes that are specific for M1$\mathrm{M} 2$ polarisation ${ }^{18,41}$. Upon treatment with M1-polarizing stimuli (LPS and IFN $\mathrm{Y}$ ) macrophages acquire a rounder shape, whereas M2-polarizing stimuli (IL-4) induce an elongated cell shape 18,42 . The extent of cell roundness was shown to correlate with the expression levels of the M1 marker iNOS and cell elongation with expression levels of the M2 marker arginase-1 ${ }^{18}$. Figure 4 confirms that IFNy stimulation increased the number of round cells, while IL-4 stimulation promoted BMDM elongation (Figure 4 and supplemental figure 3). Although transfection itself induced mild activation of macrophages ${ }^{43}$, the screen is able to detect 
both positive and negative regulators of macrophage polarisation. However, the sensitivity to detect round classified cells is higher as unstimulated cells have a more elongated morphology, resulting in physical limitations to induce an additional increase in elongation (Figure 4). A decrease in BMDM roundness, indicative of anti-inflammatory M2-polarization occurred after transfection of miR-488-5p, miR-891a-3p, miR-128-3p, and miR-34a-3p that was previously shown to target the IL-6 receptor ${ }^{44}$. Our screen identified 76 miRNAs inducing an increase in cell roundness related to pro-inflammatory M1 polarization, including miR146a and miR-155-5p which have been linked to pro-inflammatory activation ${ }^{6,45,46}$.

Nuclear translocation of NFKB is a crucial step in the coupling of extracellular stimuli to the transcriptional activation of various pro-inflammatory cytokines ${ }^{47}$. Although the $24 \mathrm{~h}$ time-point is optimal to detect morphologic changes such as cell roundness, quantification of NFKB nuclear translocation is a more acute process and earlier time-points could potentially be more informative. However, this approach allowed the quantification of more permanent effects since changes in NFKB nuclear translocation were still observed after transfection of miRNA mimics. Decreased nuclear NFKB was observed after transfection with miR-30c-2-3p and miR-34a-3p. MiRNAs increasing nuclear NFkB miR-146b-5p, miR-210-3p, miR-21-3p, and miR-590-5p. In line, silencing of miR-146a and miR-146b in dendritic cells resulted in increased NFKB activation via down-regulation of NFKB inhibitor 1 KB $\alpha{ }^{48}$. Ding et al. ${ }^{22}$ screened for miRNAs that modulate the NFKB signalling pathway upon TNF stimulation of HEK293 cells, by using a NFKB luciferase reporter. There is significant overlap in the miRNAs identified in their study and the miRNAs affecting nuclear translocation of NFKB in our screen.

Accordingly, this BMDM screen identified several novel miRNAs involved in macrophage polarisation. These results can be extrapolated to all inflammatory pathologies in which macrophages are playing crucial roles (e.g. atherosclerosis, rheumatoid arthritis). Therefore, the miRNAs identified in this screen may also be therapeutically relevant in other diseases with an inflammatory signature.

\section{$3 p$ and $5 p$ arms of mimics can show opposite effects}

Passenger strands of miRNAs were previously widely neglected ${ }^{49}$, recently it was shown that most of them are equally functional as the main strand ${ }^{49}$ and levels were found to be enriched in secreted exosomes of cardiac fibroblasts ${ }^{10}$. Both the $-3 p$ and $-5 p$ strand of many miRNAs were included in the screen, enabling determination of differences in functionality between both arms. Both miR-152-5p and miR-195-5p have pronounced effect on the number of nuclei and are well known suppressors of cell proliferation ${ }^{50}$. Strikingly, the $-3 p$ and $-5 p$ arms of these miRNAs have opposing effects on cardiac fibroblast nuclei counts. MiR$152-5 p$ mimic transfected nRCFs have only $32 \%$ of the nuclei counts compared to control transfected cells, while miR-152-3p mimic transfected fibroblasts have $162 \%$ of the nuclei compared to control (Supplementary table 1). Similar effects are observed for miR-195 where the $-3 p$ arm mimic significantly increased the number of nuclei while transfection with the $-5 p$ lead to a significant decrease. Until now, only limited studies have reported opposing effects of both miRNA arms of miR-125a and miR-28 ${ }^{51,52}$. We also observed opposing effects of miR-125a-3p (0.271 $\log _{2}$ fold change, $\left.p=0.000\right)$ and $-5 p\left(-0.337 \log _{2}\right.$ fold change, $\left.p<0.001\right)$ on proliferation of CFs. It is interesting to speculate on a biological role or mechanism, for example that one arm is destined to leave the cell and targets other cell types, or that it is a potential feedback mechanism to dampen the effect of a miRNA. 


\section{Pleiotropic effects of miRNAs}

Combining the results of the three screens has led to identification of 16 miRNAs able to affect the main phenotypic read out in each individual cellular screen. For example, miR-210 was able to increase cardiac myocyte cell size, fibroblast collagen content, as well as macrophage roundness. Thus, miR-210 potentially plays an important role in aggravating HF development by promoting cardiac hypertrophy, fibrosis, and inflammation in three cell types simultaneously.

It is important to keep in mind that observed effects were induced after miRNA mimic-library transfection, not taking into account cell type dependent miRNA expression. However, miRNAs have recently emerged as regulators of intercellular communication during physiological and pathological processes in many organs, including the heart ${ }^{10,53,54}$. Secreted extracellular vesicles (EVs) and particularly the exosomal fraction, can carry nucleic acids, including miRNAs, which can be transferred to acceptor cells and inhibit gene expression and regulate biological functions in these cells ${ }^{11,12,53,54}$. Bang et al. ${ }^{10}$ identified a miRNA/exosomemediated communication mechanism between cardiac fibroblasts and myocytes, contributing to the development of cardiac myocyte hypertrophy. Fibroblast-derived exosomes were enriched with miR-21-3p, which subsequently was transported via exosomes towards neighbouring cardiac myocytes, mediating cellular hypertrophy. In addition, Barile et al. ${ }^{11}$ showed that miR-210, identified in our screens as a miRNA able to induce significant morphological changes in all three studied cell types, is highly enriched in EVs secreted by cardiac progenitor cells and is able to inhibit apoptosis in HL-1 cardiac myocytes. In our hypertrophy screen, miR-210 was able to significantly increase cardiac myocyte cell size, suggesting that miR-210 potentially plays a role in cardiac myocyte survival and/or growth. Together, these findings suggest that miRNAs function as paracrine signalling mediators, making basal miRNA expression in a given cell type not a prerequisite for a cell-specific effect, since physiological or pathological inter-cell communication overcomes this.

\section{Limitations of the study}

In the present study we used human miRNA mimics for transfection into rat and murine primary cells. However, the effect of inter-species differences is limited as sequence analysis showed that 118 out of the 194 miRNAs display a 100\% sequence match between human and rodent and only 9 miRNA mimics showed a mismatch in the seed sequence (Supplemental table 2), potentially leading to differences in their actual mRNA-targets.

Regarding the phenotypical readouts, it is important to note that miRNA mimicinduced phenotypical changes are not per se reflecting changes in pathological process. For example, an increase in cardiomyocyte size is not per definition related to pathological hypertrophy. This requires additional analysis, for instance via the measurement of bona fide hypertrophy markers, like atrial natriuretic factor or skeletal $\alpha$-actin. This also applies to the relative Col $1 \alpha 1$ positive fibroblast cell area, the main read out for fibrosis. As shown in the supplemental Figure 2 (panels $C-D$ ), various miRNAs gave rise to a significant reduction in cell number, often along with a reduction in cell size, which is reminiscent of a pro-apoptotic effect. The reduction in cell size translates into an increase in relative Col $1 \alpha 1$ positive area per cell. Theoretically this may lead to the identification of false-positive pro-fibrotic miRNAs, once again indicating that additional analysis is required to classify such miRNAs as truly profibrotic. In a similar way, it can be argued that macrophage roundness not necessarily reflects M1 polarization, as this could also be induced by miRNAs targeting genes involved in the arrangement of the cytoskeleton or apoptosis. 
Finally, inclusion of miRNAs in our screen was based on their differential expression in hearts of different rodent models of cardiac disease, patients diagnosed with viral myocarditis, and patients undergoing cardiac transplantation as determined by micro-array analysis or by deep sequencing of small RNAs. Generally speaking, these cardiac diseases are characterized by a marked inflammatory component, resulting in a bias for miRNAs related to inflammation. Nonetheless, many of the selected miRNAs were shown to influence hypertrophy and fibrosis as well, implying that these three processes are intricately linked.

\section{Conclusions}

We performed a high throughput phenotypical screen in three relevant primary (cardiac) cell types, thereby identifying miRNAs involved in hypertrophy, fibrosis and inflammation. We conclude that our multi-cell type high-throughput screening approach of unstimulated and stimulated cells, allows in-depth analysis of the effect of HF-associated miRNAs on cardiac pathophysiology. Screening for hypertrophy, fibrosis, and inflammation simultaneously resulted in a complete description of miRNA-induced effects in the development of HF. In this way we confirmed roles of well-known miRNAs, but also identified many miRNAs new to the HF-research field. These miRNAs, especially the miRNAs that affected multiple HFunderlying processes, are interesting candidates for further in-depth investigation as therapeutic targets.

\section{Acknowledgements}

Authors would like to thank Sarah Weiss, Andrew Walsh, Steffen Schubert, Kartini Kochar, Michael Brehm and Arend Grosse for excellent scientific and technical support. The research leading to these results has received funding from the European Union Commission's Seventh Framework program under grant the Marie-Curie Industry Academy Pathways and Partnerships (CARDIOMIR) N²85991 (screen of inflammation and hypertrophy), N 602904 (FIBROTARGETS) (previous animal work and fibroblast screen), and FP7-Health-2013Innovations-1 N 602156 (HECATOS) (bio-informatical analysis). 


\section{References}

1. Burchfield JS, Xie M and Hill JA. Pathological ventricular remodelling: mechanisms: part 1 of 2. Circulation. 2013;128:388-400.

2. Lloyd-Jones D, Adams RJ, Brown TM, Carnethon M, Dai S, De Simone G, Ferguson TB, Ford E, Furie K, Gillespie C, Go A, Greenlund K, Haase N, Hailpern S, Ho PM, Howard V, Kissela B, Kittner S, Lackland D, Lisabeth L, Marelli A, McDermott MM, Meigs J, Mozaffarian D, Mussolino M, Nichol G, Roger VL, Rosamond W, Sacco R, Sorlie P, Stafford R, Thom T, Wasserthiel-Smoller S, Wong ND, Wylie-Rosett J, American Heart Association Statistics $C$ and Stroke Statistics S. Executive summary: heart disease and stroke statistics--2010 update: a report from the American Heart Association. Circulation. 2010;121:94854.

3. Heymans S, Hirsch E, Anker SD, Aukrust P, Balligand JL, Cohen-Tervaert JW, Drexler H, Filippatos G, Felix SB, Gullestad L, Hilfiker-Kleiner D, Janssens S, Latini R, Neubauer G, Paulus WJ, Pieske B, Ponikowski P, Schroen B, Schultheiss HP, Tschope C, Van Bilsen M, Zannad F, McMurray J and Shah AM. Inflammation as a therapeutic target in heart failure? A scientific statement from the Translational Research Committee of the Heart Failure Association of the European Society of Cardiology. Eur J Heart Fail. 2009;11:119-29.

4. Ikeda S, He A, Kong SW, Lu J, Bejar R, Bodyak N, Lee KH, Ma Q, Kang PM, Golub TR and Pu WT. MicroRNA1 negatively regulates expression of the hypertrophy-associated calmodulin and Mef2a genes. Mol Cell Biol. 2009;29:2193-204.

5. van Rooij E, Sutherland LB, Thatcher JE, DiMaio JM, Naseem RH, Marshall WS, Hill JA and Olson EN. Dysregulation of microRNAs after myocardial infarction reveals a role of miR-29 in cardiac fibrosis. Proc Natl Acad Sci U S A. 2008;105:13027-32.

6. Corsten MF, Papageorgiou A, Verhesen W, Carai P, Lindow M, Obad S, Summer G, Coort SL, Hazebroek M, van Leeuwen R, Gijbels MJ, Wijnands E, Biessen EA, De Winther MP, Stassen FR, Carmeliet P, Kauppinen S, Schroen B and Heymans S. MicroRNA profiling identifies microRNA-155 as an adverse mediator of cardiac injury and dysfunction during acute viral myocarditis. Circulation research. 2012;111:415-25.

7. Ha M and Kim VN. Regulation of microRNA biogenesis. Nat Rev Mol Cell Biol. 2014;15:509-24.

8. Kunz M, Xiao K, Liang C, Viereck J, Pachel C, Frantz S, Thum T and Dandekar T. Bioinformatics of cardiovascular miRNA biology. J Mol Cell Cardiol. 2014.

9. Latronico MV and Condorelli G. MicroRNAs and cardiac pathology. Nature reviews Cardiology. 2009;6:419-29.

10. Bang C, Batkai S, Dangwal S, Gupta SK, Foinquinos A, Holzmann A, Just A, Remke J, Zimmer K, Zeug A, Ponimaskin E, Schmiedl A, Yin X, Mayr M, Halder R, Fischer A, Engelhardt S, Wei Y, Schober A, Fiedler J and Thum T. Cardiac fibroblast-derived microRNA passenger strand-enriched exosomes mediate cardiomyocyte hypertrophy. J Clin Invest. 2014;124:2136-46.

11. Barile L, Lionetti V, Cervio E, Matteucci M, Gherghiceanu M, Popescu LM, Torre T, Siclari F, Moccetti T and Vassalli G. Extracellular vesicles from human cardiac progenitor cells inhibit cardiomyocyte apoptosis and improve cardiac function after myocardial infarction. Cardiovasc Res. 2014;103:530-41.

12. Cervio E, Barile L, Moccetti T and Vassalli G. Exosomes for Intramyocardial Intercellular Communication. Stem cells international. 2015;2015:482171.

13. Van Aelst LN, Summer G, Li S, Gupta SK, Heggermont W, De Vusser K, Carai P, Naesens M, Van Cleemput J, Van de Werf F, Vanhaecke J, Thum T, Waer M, Papageorgiou AP, Schroen B and Heymans S. RNA Profiling in Human and Murine Transplanted Hearts: Identification and Validation of Therapeutic Targets for Acute Cardiac and Renal Allograft Rejection. Am J Transplant. 2015.

14. De Windt LJ, Willemsen PH, Popping S, Van der Vusse GJ, Reneman RS and Van Bilsen M. Cloning and cellular distribution of a group II phospholipase A2 expressed in the heart. Journal of molecular and cellular cardiology. 1997;29:2095-106.

15. Heymans S, Corsten MF, Verhesen W, Carai P, van Leeuwen RE, Custers K, Peters T, Hazebroek M, Stoger L, Wijnands E, Janssen BJ, Creemers EE, Pinto YM, Grimm D, Schurmann N, Vigorito E, Thum T, Stassen F, Yin X, Mayr M, de Windt LJ, Lutgens E, Wouters K, de Winther MP, Zacchigna S, Giacca M, van Bilsen M, Papageorgiou AP and Schroen B. Macrophage microRNA-155 promotes cardiac hypertrophy and failure. Circulation. 2013;128:1420-32.

16. He Y, Huang C, Lin X and Li J. MicroRNA-29 family, a crucial therapeutic target for fibrosis diseases. Biochimie. 2013;95:1355-9.

17. Pan Z, Sun X, Shan H, Wang N, Wang J, Ren J, Feng S, Xie L, Lu C, Yuan Y, Zhang Y, Wang Y, Lu Y and Yang B. MicroRNA-101 inhibited postinfarct cardiac fibrosis and improved left ventricular compliance via the FBJ osteosarcoma oncogene/transforming growth factor-beta1 pathway. Circulation. 2012;126:840-50. 
18. McWhorter FY, Wang T, Nguyen P, Chung T and Liu WF. Modulation of macrophage phenotype by cell shape. Proc Natl Acad Sci U S A. 2013;110:17253-8.

19. Graff JW, Dickson AM, Clay G, McCaffrey AP and Wilson ME. Identifying functional microRNAs in macrophages with polarized phenotypes. J Biol Chem. 2012;287:21816-25.

20. Taganov KD, Boldin MP, Chang KJ and Baltimore D. NF-kappaB-dependent induction of microRNA miR146, an inhibitor targeted to signaling proteins of innate immune responses. Proc Natl Acad Sci U S A. 2006;103:12481-6.

21. Jentzsch C, Leierseder S, Loyer X, Flohrschutz I, Sassi Y, Hartmann D, Thum T, Laggerbauer B and Engelhardt S. A phenotypic screen to identify hypertrophy-modulating microRNAs in primary cardiomyocytes. J Mol Cell Cardiol. 2012;52:13-20.

22. Ding J, Huang S, Wang $Y$, Tian $Q$, Zha R, Shi H, Wang Q, Ge C, Chen T, Zhao Y, Liang L, Li J and He X. Genome-wide screening reveals that miR-195 targets the TNF-alpha/NF-kappaB pathway by downregulating IkappaB kinase alpha and TAB3 in hepatocellular carcinoma. Hepatology. 2013;58:654-66.

23. Eulalio A and Mano M. MicroRNA Screening and the Quest for Biologically Relevant Targets. J Biomol Screen. 2015.

24. Eimre M, Paju K, Pelloux S, Beraud N, Roosimaa M, Kadaja L, Gruno M, Peet N, Orlova E, Remmelkoor R, Piirsoo A, Saks V and Seppet E. Distinct organization of energy metabolism in HL-1 cardiac cell line and cardiomyocytes. Biochim Biophys Acta. 2008;1777:514-24.

25. Hartley JW, Evans LH, Green KY, Naghashfar Z, Macias AR, Zerfas PM and Ward JM. Expression of infectious murine leukemia viruses by RAW264.7 cells, a potential complication for studies with a widely used mouse macrophage cell line. Retrovirology. 2008;5:1.

26. Fiedler LR, Maifoshie E and Schneider MD. Mouse models of heart failure: cell signaling and cell survival. Current topics in developmental biology. 2014;109:171-247.

27. Song DW, Ryu JY, Kim JO, Kwon EJ and Kim do H. The miR-19a/b family positively regulates cardiomyocyte hypertrophy by targeting atrogin-1 and MuRF-1. The Biochemical journal. 2014;457:151-62.

28. Miao Y, Chen H and Li M. MiR-19a overexpression contributes to heart failure through targeting ADRB1. International journal of clinical and experimental medicine. 2015;8:642-9.

29. Leptidis S, El Azzouzi H, Lok SI, de Weger R, Olieslagers S, Kisters N, Silva GJ, Heymans S, Cuppen E, Berezikov $E$, De Windt $L$ and da Costa Martins $P$. A deep sequencing approach to uncover the miRNOME in the human heart. PLoS One. 2013;8:e57800.

30. Goren Y, Kushnir M, Zafrir B, Tabak S, Lewis BS and Amir O. Serum levels of microRNAs in patients with heart failure. Eur J Heart Fail. 2012;14:147-54.

31. Taurino C, Miller WH, McBride MW, McClure JD, Khanin R, Moreno MU, Dymott JA, Delles C and Dominiczak AF. Gene expression profiling in whole blood of patients with coronary artery disease. Clin Sci (Lond). 2010;119:335-43.

32. Assomull RG, Prasad SK, Lyne J, Smith G, Burman ED, Khan M, Sheppard MN, Poole-Wilson PA and Pennell DJ. Cardiovascular magnetic resonance, fibrosis, and prognosis in dilated cardiomyopathy. J Am Coll Cardiol. 2006;48:1977-85.

33. Booz GW and Baker KM. Molecular signalling mechanisms controlling growth and function of cardiac fibroblasts. Cardiovasc Res. 1995;30:537-43.

34. Voorhoeve PM, le Sage C, Schrier M, Gillis AJ, Stoop H, Nagel R, Liu YP, van Duijse J, Drost J, Griekspoor A, Zlotorynski E, Yabuta N, De Vita G, Nojima H, Looijenga LH and Agami R. A genetic screen implicates miRNA-372 and miRNA-373 as oncogenes in testicular germ cell tumors. Cell. 2006;124:1169-81.

35. Chen Y, Song YX and Wang ZN. The microRNA-148/152 family: multi-faceted players. Mol Cancer. 2013;12:43.

36. Rissland OS, Hong SJ and Bartel DP. MicroRNA destabilization enables dynamic regulation of the miR-16 family in response to cell-cycle changes. Mol Cell. 2011;43:993-1004.

37. Duisters RF, Tijsen AJ, Schroen B, Leenders JJ, Lentink V, van der Made I, Herias V, van Leeuwen RE, Schellings MW, Barenbrug P, Maessen JG, Heymans S, Pinto YM and Creemers EE. miR-133 and miR-30 regulate connective tissue growth factor: implications for a role of microRNAs in myocardial matrix remodeling. Circ Res. 2009;104:170-8, 6p following 178.

38. Thum T, Gross C, Fiedler J, Fischer T, Kissler S, Bussen M, Galuppo P, Just S, Rottbauer W, Frantz S, Castoldi M, Soutschek J, Koteliansky V, Rosenwald A, Basson MA, Licht JD, Pena JT, Rouhanifard SH, Muckenthaler MU, Tuschl T, Martin GR, Bauersachs J and Engelhardt S. MicroRNA-21 contributes to myocardial disease by stimulating MAP kinase signalling in fibroblasts. Nature. 2008;456:980-4.

39. Wang J, Huang W, Xu R, Nie Y, Cao X, Meng J, Xu X, Hu S and Zheng Z. MicroRNA-24 regulates cardiac fibrosis after myocardial infarction. J Cell Mol Med. 2012;16:2150-60.

40. Murray PJ, Allen JE, Biswas SK, Fisher EA, Gilroy DW, Goerdt S, Gordon S, Hamilton JA, Ivashkiv LB, Lawrence T, Locati M, Mantovani A, Martinez FO, Mege JL, Mosser DM, Natoli G, Saeij JP, Schultze JL, 
Shirey KA, Sica A, Suttles J, Udalova I, van Ginderachter JA, Vogel SN and Wynn TA. Macrophage activation and polarization: nomenclature and experimental guidelines. Immunity. 2014;41:14-20.

41. Tyteca D, Nishino T, Debaix H, Van Der Smissen P, N'Kuli F, Hoffmann D, Cnops Y, Rabolli V, van Loo G, Beyaert R, Huaux F, Devuyst $O$ and Courtoy PJ. Regulation of macrophage motility by the water channel aquaporin-1: crucial role of MO/M2 phenotype switch. PLoS One. 2015;10:e0117398.

42. Vereyken EJ, Heijnen PD, Baron W, de Vries EH, Dijkstra CD and Teunissen CE. Classically and alternatively activated bone marrow derived macrophages differ in cytoskeletal functions and migration towards specific CNS cell types. J Neuroinflammation. 2011;8:58.

43. Carralot JP, Kim TK, Lenseigne B, Boese AS, Sommer P, Genovesio A and Brodin P. Automated highthroughput siRNA transfection in raw 264.7 macrophages: a case study for optimization procedure. $J$ Biomol Screen. 2009;14:151-60.

44. Rokavec M, Oner MG, Li H, Jackstadt R, Jiang L, Lodygin D, Kaller M, Horst D, Ziegler PK, Schwitalla S, Slotta-Huspenina J, Bader FG, Greten FR and Hermeking H. IL-6R/STAT3/miR-34a feedback loop promotes EMT-mediated colorectal cancer invasion and metastasis. J Clin Invest. 2014;124:1853-67.

45. Cheng Y, Kuang W, Hao Y, Zhang D, Lei M, Du L, Jiao H, Zhang X and Wang F. Downregulation of miR-27a* and miR-532-5p and upregulation of miR-146a and miR-155 in LPS-induced RAW264.7 macrophage cells. Inflammation. 2012;35:1308-13.

46. He Y, Sun X, Huang C, Long XR, Lin X, Zhang L, Lv XW and Li J. MiR-146a regulates IL-6 production in lipopolysaccharide-induced RAW264.7 macrophage cells by inhibiting Notch1. Inflammation. 2014;37:7182.

47. Trask OJ, Jr. Nuclear Factor Kappa B (NF-kappaB) Translocation Assay Development and Validation for High Content Screening. In: G. S. Sittampalam, N. P. Coussens, H. Nelson, M. Arkin, D. Auld, C. Austin, B. Bejcek, M. Glicksman, J. Inglese, P. W. Iversen, Z. Li, J. McGee, O. McManus, L. Minor, A. Napper, J. M. Peltier, T. Riss, O. J. Trask, Jr. and J. Weidner, eds. Assay Guidance Manual Bethesda (MD); 2004.

48. Park H, Huang X, Lu C, Cairo MS and Zhou X. MicroRNA-146a and microRNA-146b regulate human dendritic cell apoptosis and cytokine production by targeting TRAF6 and IRAK1 proteins. $J$ Biol Chem. 2015;290:2831-41.

49. Hirt MN, Werner T, Indenbirken D, Alawi M, Demin P, Kunze AC, Stenzig J, Starbatty J, Hansen A, Fiedler J, Thum $T$ and Eschenhagen T. Deciphering the microRNA signature of pathological cardiac hypertrophy by engineered heart tissue- and sequencing-technology. J Mol Cell Cardiol. 2015;81:1-9.

50. Huang H, Hu M, Li P, Lu C and Li M. Mir-152 inhibits cell proliferation and colony formation of CD133(+) liver cancer stem cells by targeting KIT. Tumour Biol. 2015;36:921-8.

51. Jiang L, Huang Q, Zhang S, Zhang Q, Chang J, Qiu X and Wang E. Hsa-miR-125a-3p and hsa-miR-125a-5p are downregulated in non-small cell lung cancer and have inverse effects on invasion and migration of lung cancer cells. BMC Cancer. 2010;10:318.

52. Almeida MI, Nicoloso MS, Zeng L, Ivan C, Spizzo R, Gafa R, Xiao L, Zhang X, Vannini I, Fanini F, Fabbri M, Lanza G, Reis RM, Zweidler-McKay PA and Calin GA. Strand-specific miR-28-5p and miR-28-3p have distinct effects in colorectal cancer cells. Gastroenterology. 2012;142:886-896 e9.

53. Valadi H, Ekstrom K, Bossios A, Sjostrand M, Lee JJ and Lotvall JO. Exosome-mediated transfer of mRNAs and microRNAs is a novel mechanism of genetic exchange between cells. Nat Cell Biol. 2007;9:654-9.

54. Ong SG, Lee WH, Huang M, Dey D, Kodo K, Sanchez-Freire V, Gold JD and Wu JC. Cross talk of combined gene and cell therapy in ischemic heart disease: role of exosomal microRNA transfer. Circulation. 2014;130:S60-9. 


\section{Supplemental Figures and Tables}

A
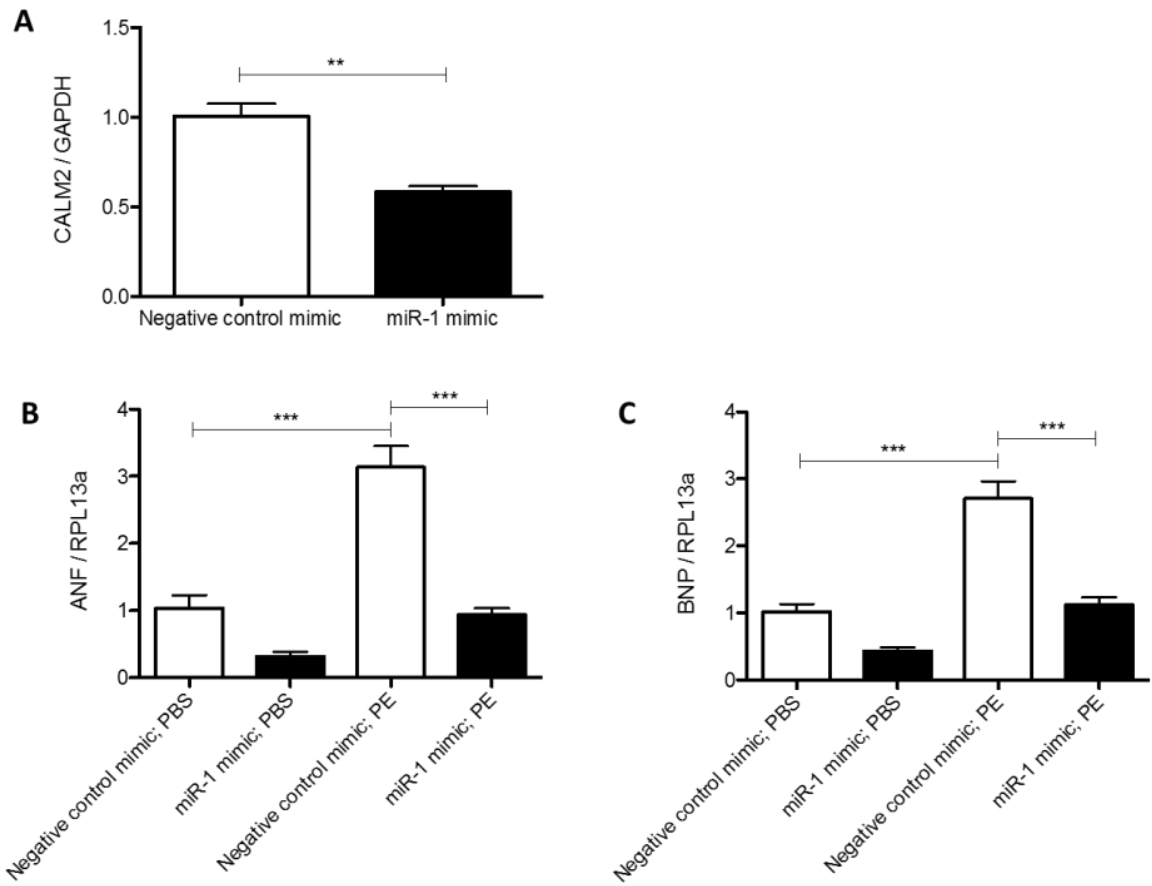

Supplemental Figure 1: A) RT-PCR analysis showed that transfection of miR-1 mimic in nRCMs resulted in decreased expression of direct target calmodulin 2 (CALM2). Unpaired $t$-test ${ }^{* *} p<0.01$. Stimulation of $n R C M s$ with PE resulted in upregulation of hypertrophic markers ANF (B) and BNP (C) and transfection of anti-hypertrophic miRNA miR-1 prevented the PE-induced upregulation of both ANF and BNP expression significantly. One-way ANOVA ${ }^{* * *} p<0.001$. 

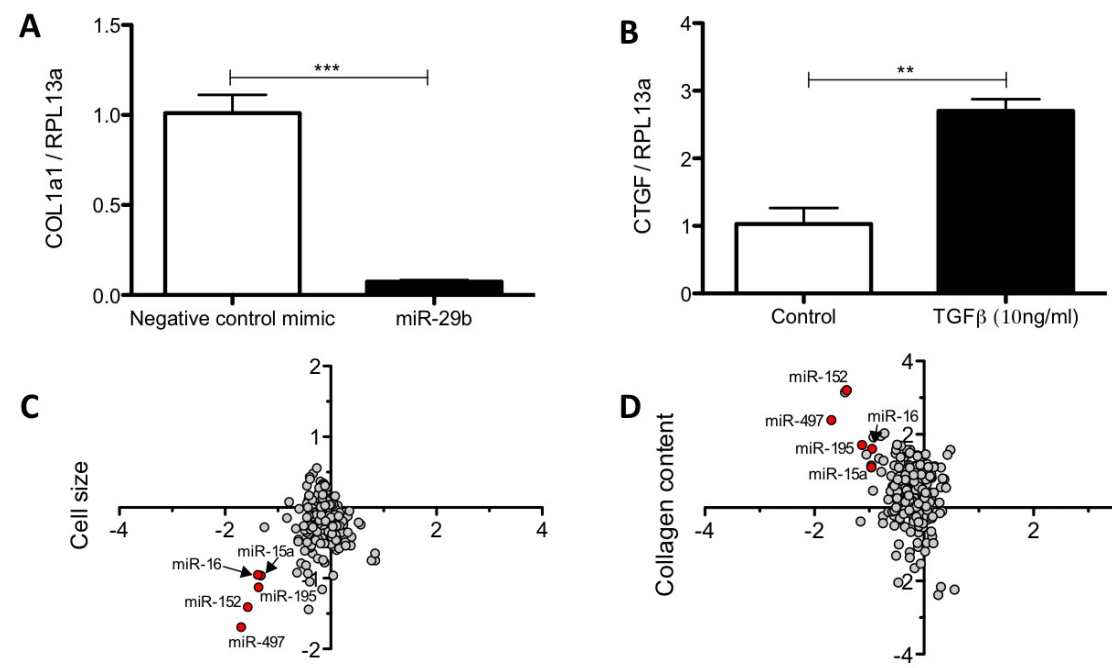

Number of nuclei

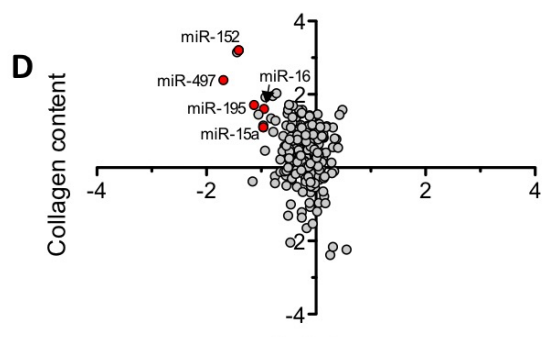

Cell size

Supplemental Figure 2: A) RT-PCR quantification showed that transfection of $n R C F s$ with a miR-29b mimic resulted in a significant decrease in mRNA expression of direct target COL1 1 1. B) Stimulation of nRCFs with TGFB resulted in increased transcript levels of CTGF. Unpaired T-Test ${ }^{*} P<0.05$. C -D) Several miRNAs gave rise to a substantial reduction in cell number and simultaneously reduced cell area, suggesting that these miRNAs promote apoptosis. This may have an impact on the relative Col1 11 positive area as well. 
A

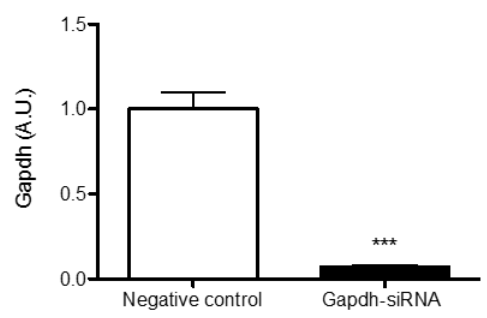

C

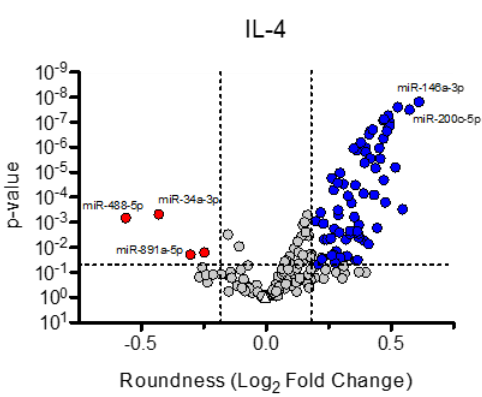

B

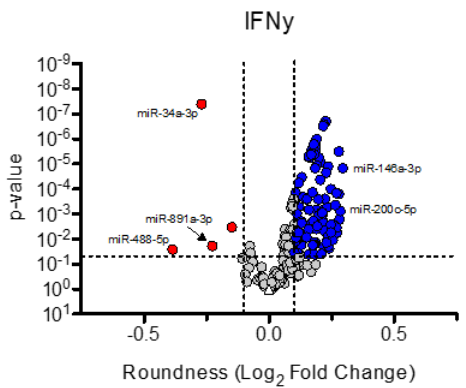

D

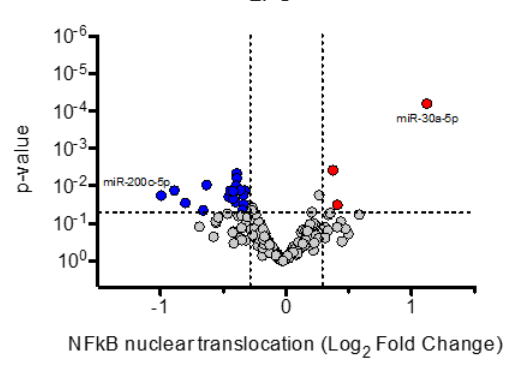

Supplemental 3: A) Transfection of BMDMs with a siRNA for Gapdh resulted in a 93\% decrease in mRNA levels. Vulcano plots showing $\log _{2}$ fold change in cell roundness in macrophages polarised towards B) M1 (IFNY) and C) M2 (IL-4) and in D) NFKB nuclear translocation in LPS-stimulated conditions compared to negative control mimic transfected BMDMs. MiRNAs able to increase and decrease BMDM roundness and NFKB nuclear translocation are shown in blue and red, respectively.

Supplemental Table 2. MiRNAs that do not have a conserved seed sequence between human and rat.

\begin{tabular}{lll}
\hline miRNA & Human sequence & Rat sequence \\
\hline hsa-miR-222-5p & CUCAGUAGCCAGUGUAGAUCCU & GGCUCAGUAGCCAGUGUAGAU \\
hsa-miR-450b-3p & UUGGGAUCAUUUUGCAUCCAUA & AUUGGGGACGCUUCGCAUUCA \\
hsa-miR-30b-3p & CUGGGAGGUGGAUGUUUACUUC & CUGGGAUGUGGAUGUUUACGUC \\
hsa-miR-345-5p & GCUGACUCCUAGUCCAGGGCUC & UGCUGACCCCUAGUCCAGUGC \\
hsa-miR-146b-3p & UGCCCUGUGGACUCAGUUCUGG & CCUAGGGACUCAGUUCUGGUG \\
hsa-miR-214-5p & UGCCUGUCUACACUUGCUGUGC & AGAGUUGUCAUGUGUCU \\
hsa-miR-210-5p & AGCCCCUGCCCACCGCACACUG & AGCCACUGCCCACAGCACACUG \\
hsa-miR-511-5p & GUGUCUUUUGCUCUGCAGUCA & CAUGCCUUUUGCUCUGCACUC \\
hsa-miR-135a-3p & UAUAGGGAUUGGAGCCGUGGCG & UGUAGGGAUGGAAGCCAUGAA \\
\hline
\end{tabular}


Supplemental Table 1. Effects of heart failure-associated miRNA mimics upon transfection, on the main read outs for each screen. Values are represented as relative $\log _{2}$ fold change over negative control mimic-transfected cells. MiRNAs were selected for high throughput phenotypical screening based on their differential expression in the hearts of patients diagnosed with viral myocarditis (Human VM), patients undergoing cardiac transplantation (Human TX), and in animal models of inflammatory cardiac disease, such as murine viral myocarditis (Mouse VM), murine cardiac transplant (Mouse TX), and rat metabolic syndrome (ZSF1). In the table Mouse VM $=A$, Mouse $T X=B, Z S F 1=C$, Human $V M=D$ and Human $T X=E$.

\begin{tabular}{|c|c|c|c|c|c|c|c|c|c|c|c|}
\hline \multirow[b]{2}{*}{ miRNA } & \multirow{2}{*}{$\begin{array}{l}\text { miRNA } \\
\text { Profiling } \\
\text { study }\end{array}$} & \multicolumn{2}{|c|}{$\begin{array}{l}\text { Cardiomyocyte } \\
\text { Cell Size }\end{array}$} & \multicolumn{2}{|c|}{$\begin{array}{l}\text { Fibroblast } \\
\text { Collagen content }\end{array}$} & \multicolumn{2}{|c|}{ N\# of Nuclei } & \multicolumn{2}{|c|}{$\begin{array}{l}\text { Macrophage } \\
\text { Roundness }\end{array}$} & \multicolumn{2}{|c|}{ Nuclear NFkB } \\
\hline & & $\log 2 \mathrm{Fc}$ & Pval & $\log 2 \mathrm{Fc}$ & Pval & $\log 2 \mathrm{Fc}$ & Pval & $\begin{array}{l}\log 2 \\
\text { Fc }\end{array}$ & Pval & $\log 2 \mathrm{Fc}$ & Pval \\
\hline let-7a-5p & $A, C$ & $-0,05$ & 0,59 & 0,58 & 0,00 & 0,02 & 0,58 & 0,04 & 0,43 & $-0,04$ & 0,74 \\
\hline let-7b-3p & A & 0,12 & 0,39 & 0,17 & 0,14 & $-0,15$ & 0,04 & 0,24 & 0,05 & 0,29 & 0,01 \\
\hline let-7b-5p & $A, C$ & $-0,09$ & 0,55 & 0,36 & 0,02 & $-0,02$ & 0,76 & 0,16 & 0,09 & $-0,10$ & 0,60 \\
\hline let-7c-3p & $A$ & $-0,25$ & 0,01 & $-1,13$ & 0,00 & $-0,09$ & 0,04 & 0,34 & 0,00 & 0,42 & 0,03 \\
\hline let-7c-5p & $A, C$ & 0,12 & 0,06 & 0,43 & 0,02 & 0,11 & 0,01 & 0,08 & 0,08 & $-0,08$ & 0,52 \\
\hline let-7d-5p & A & $-0,08$ & 0,46 & 0,43 & 0,02 & 0,03 & 0,39 & $-0,06$ & 0,05 & $-0,12$ & 0,37 \\
\hline let-7e-5p & $A$ & $-0,09$ & 0,29 & 1,39 & 0,00 & 0,13 & 0,00 & 0,16 & 0,14 & 0,65 & 0,37 \\
\hline let-7f-5p & $A$ & $-0,02$ & 0,94 & 0,71 & 0,00 & $-0,06$ & 0,20 & 0,02 & 0,51 & $-0,13$ & 0,32 \\
\hline let-7g-5p & $A$ & $-0,17$ & 0,06 & 0,27 & 0,08 & $-0,06$ & 0,16 & 0,02 & 0,45 & $-0,15$ & 0,12 \\
\hline miR-1 & A & $-0,50$ & 0,01 & 1,89 & 0,00 & $-0,56$ & 0,00 & 0,15 & 0,05 & 0,00 & 0,92 \\
\hline miR-100-5p & $A$ & $-0,04$ & 0,77 & $-0,21$ & 0,67 & $-0,02$ & 0,78 & 0,10 & 0,12 & $-0,16$ & 0,25 \\
\hline miR-101-3p & $A$ & $-0,25$ & 0,00 & 0,16 & 0,16 & $-0,35$ & 0,00 & 0,25 & 0,03 & 0,01 & 0,86 \\
\hline miR-103a-2-5p & B & $-0,12$ & 0,33 & $-1,71$ & 0,00 & $-0,35$ & 0,00 & 0,30 & 0,00 & 0,12 & 0,12 \\
\hline miR-103a-3p & $\mathrm{B}, \mathrm{C}$ & $-0,51$ & 0,00 & 0,31 & 0,04 & $-0,40$ & 0,00 & 0,12 & 0,15 & 0,08 & 0,50 \\
\hline miR-103b & B & $-0,76$ & 0,00 & 0,52 & 0,01 & $-0,39$ & 0,00 & 0,31 & 0,01 & 0,35 & 0,13 \\
\hline miR-106a-3p & B & 0,20 & 0,18 & 0,51 & 0,01 & $-0,42$ & 0,00 & 0,07 & 0,29 & $-0,03$ & 0,89 \\
\hline miR-106a-5p & B & $-0,35$ & 0,02 & 0,52 & 0,01 & $-0,06$ & 0,29 & 0,22 & 0,08 & 0,07 & 0,65 \\
\hline miR-106b-5p & B & $-0,24$ & 0,25 & 0,62 & 0,00 & $-0,21$ & 0,01 & 0,14 & 0,06 & $-0,17$ & 0,31 \\
\hline miR-107 & $B, C$ & $-0,04$ & 0,76 & $-0,16$ & 0,88 & $-0,40$ & 0,00 & 0,08 & 0,08 & $-0,22$ & 0,15 \\
\hline$m i R-10 a-5 p$ & C & $-0,05$ & 0,67 & $-0,74$ & 0,01 & 0,06 & 0,02 & 0,30 & 0,03 & 0,14 & 0,16 \\
\hline miR-10b-5p & $A$ & 0,10 & 0,46 & $-0,22$ & 0,63 & $-0,05$ & 0,49 & 0,24 & 0,05 & 0,05 & 0,75 \\
\hline miR-1224-3p & $B, A$ & 0,02 & 0,57 & 0,52 & 0,00 & $-0,09$ & 0,26 & 0,08 & 0,14 & 0,00 & 0,93 \\
\hline miR-1224-5p & $B, A$ & $-0,29$ & 0,02 & $-0,36$ & 0,27 & 0,01 & 0,72 & $-0,01$ & 0,70 & $-0,15$ & 0,11 \\
\hline miR-1246 & $\mathrm{D}$ & $-0,36$ & 0,00 & $-0,06$ & 0,73 & 0,08 & 0,18 & $-0,08$ & 0,08 & $-0,07$ & 0,69 \\
\hline $\operatorname{miR}-125 a-3 p$ & B & $-0,04$ & 0,58 & 0,86 & 0,00 & 0,27 & 0,00 & 0,07 & 0,07 & $-0,01$ & 1,00 \\
\hline miR-125a-5p & $A, B$ & 0,37 & 0,00 & 1,51 & 0,08 & $-0,34$ & 0,00 & 0,06 & 0,22 & 0,15 & 0,14 \\
\hline miR-125b-5p & $A, E, C$ & 0,16 & 0,09 & 0,71 & 0,00 & $-0,20$ & 0,00 & 0,06 & 0,30 & $-0,02$ & 0,99 \\
\hline miR-126-3p & $A, B$ & $-0,33$ & 0,02 & 0,92 & 0,00 & $-0,26$ & 0,01 & 0,02 & 0,74 & 0,39 & 0,04 \\
\hline miR-126-5p & A & $-0,18$ & 0,06 & $-0,12$ & 0,99 & 0,21 & 0,00 & 0,20 & 0,02 & 0,05 & 0,47 \\
\hline miR-128-1-5p & B & 0,04 & 0,31 & 0,87 & 0,00 & 0,03 & 0,49 & $-0,03$ & 0,59 & $-0,15$ & 0,24 \\
\hline miR-128-2-5p & B & 0,05 & 0,66 & $-0,03$ & 0,70 & 0,13 & 0,04 & 0,02 & 0,68 & $-0,24$ & 0,13 \\
\hline miR-128-3p & B & $-0,10$ & 0,62 & $-0,58$ & 0,03 & $-0,06$ & 0,14 & $-0,21$ & 0,01 & $-0,17$ & 0,10 \\
\hline miR-130a-3p & $A$ & $-0,40$ & 0,03 & 0,09 & 0,27 & $-0,26$ & 0,00 & 0,22 & 0,01 & 0,19 & 0,10 \\
\hline miR-130b-3p & $A$ & $-0,29$ & 0,09 & 0,16 & 0,15 & $-0,25$ & 0,00 & 0,19 & 0,08 & 0,01 & 0,88 \\
\hline $\operatorname{miR}-133 a-3 p$ & $A$ & $-0,04$ & 0,80 & 0,73 & 0,00 & $-0,13$ & 0,05 & 0,20 & 0,05 & 0,07 & 0,59 \\
\hline miR-133a-5p & A & 0,18 & 0,19 & 0,49 & 0,01 & $-0,45$ & 0,00 & 0,03 & 0,17 & $-0,11$ & 0,29 \\
\hline miR-133b & $A, C$ & $-0,40$ & 0,00 & 0,75 & 0,00 & $-0,10$ & 0,02 & 0,19 & 0,00 & $-0,04$ & 0,79 \\
\hline miR-135a-3p & A & $-0,13$ & 0,22 & 1,00 & 0,00 & $-0,13$ & 0,01 & 0,09 & 0,09 & $-0,22$ & 0,12 \\
\hline miR-135b-5p & $A, C$ & $-0,07$ & 0,24 & $-0,59$ & 0,04 & $-0,46$ & 0,00 & 0,14 & 0,12 & $-0,10$ & 0,48 \\
\hline miR-139-3p & $A, C, B$ & 0,02 & 0,75 & 0,29 & 0,04 & 0,08 & 0,13 & 0,07 & 0,04 & 0,14 & 0,36 \\
\hline miR-139-5p & $A, C, B$ & 0,01 & 0,87 & $-0,39$ & 0,22 & $-0,11$ & 0,05 & 0,17 & 0,00 & 0,07 & 0,43 \\
\hline$m i R-140-3 p$ & $\mathrm{C}$ & $-0,04$ & 0,66 & $-0,45$ & 0,15 & 0,18 & 0,00 & 0,09 & 0,00 & $-0,09$ & 0,56 \\
\hline miR-142-3p & $A, E$ & $-0,39$ & 0,00 & $-0,44$ & 0,21 & $-0,16$ & 0,11 & 0,13 & 0,11 & $-0,02$ & 0,96 \\
\hline$m i R-142-5 p$ & $A, E$ & $-0,01$ & 0,95 & 0,32 & 0,03 & $-0,13$ & 0,05 & 0,11 & 0,12 & $-0,06$ & 0,69 \\
\hline miR-143-3p & $B, A, C$ & $-0,06$ & 0,46 & $-0,13$ & 0,99 & 0,05 & 0,30 & $-0,14$ & 0,06 & $-0,04$ & 0,82 \\
\hline miR-145-3p & $B, A$ & $-0,39$ & 0,00 & 0,96 & 0,00 & $-0,27$ & 0,00 & 0,28 & 0,04 & 0,36 & 0,00 \\
\hline miR-145-5p & $B, A$ & 0,09 & 0,67 & 0,88 & 0,00 & $-0,01$ & 0,92 & 0,06 & 0,30 & $-0,06$ & 0,73 \\
\hline miR-146a-3p & A, B & 0,22 & 0,15 & $-0,30$ & 0,38 & $-0,15$ & 0,00 & 0,40 & 0,00 & 0,17 & 0,21 \\
\hline miR-146a-5p & $A, B, C$ & 0,50 & 0,42 & 1,07 & 0,00 & 0,26 & 0,00 & 0,01 & 0,89 & $-0,12$ & 0,40 \\
\hline miR-146b-3p & $A, B$ & $-0,17$ & 0,02 & 0,19 & 0,11 & $-0,11$ & 0,15 & $-0,04$ & 0,65 & $-0,15$ & 0,39 \\
\hline miR-146b-5p & $A, B, C$ & $-0,05$ & 0,72 & 1,01 & 0,00 & 0,32 & 0,00 & 0,29 & 0,04 & 0,61 & 0,02 \\
\hline miR-148a-3p & B & $-0,36$ & 0,04 & $-0,30$ & 0,45 & 0,84 & 0,00 & 0,19 & 0,08 & $-0,08$ & 0,62 \\
\hline miR-148b-3p & $\mathrm{C}$ & $-0,16$ & 0,05 & $-0,48$ & 0,10 & 0,77 & 0,00 & 0,38 & 0,01 & 0,21 & 0,05 \\
\hline miR-149-5p & $A, E$ & $-0,12$ & 0,30 & 0,71 & 0,00 & $-0,12$ & 0,01 & 0,22 & 0,00 & 0,05 & 0,59 \\
\hline
\end{tabular}




\begin{tabular}{|c|c|c|c|c|c|c|c|c|c|c|c|}
\hline miR-150-5p & C & $-0,06$ & 0,61 & 0,40 & 0,02 & $-0,18$ & 0,00 & 0,07 & 0,45 & $-0,03$ & 0,83 \\
\hline miR-151a-3p & B & $-0,15$ & 0,26 & $-1,03$ & 0,00 & $-0,33$ & 0,00 & $-0,11$ & 0,09 & $-0,04$ & 0,71 \\
\hline miR-151a-5p & $A, B$ & $-0,14$ & 0,17 & 1,51 & 0,00 & $-0,10$ & 0,02 & $-0,07$ & 0,43 & 0,22 & 0,05 \\
\hline miR-152-3p & A & $-0,24$ & 0,18 & $-0,18$ & 0,83 & 0,84 & 0,00 & 0,27 & 0,00 & 0,27 & 0,03 \\
\hline miR-152-5p & A & 0,23 & 0,02 & 3,14 & 0,00 & $-1,57$ & 0,00 & 0,29 & 0,02 & $-0,02$ & 0,95 \\
\hline miR-153-3p & $A$ & 0,48 & 0,35 & 0,39 & 0,03 & $-0,49$ & 0,00 & 0,31 & 0,00 & 0,19 & 0,18 \\
\hline miR-155-5p & $A, C, D$ & $-0,12$ & 0,08 & 0,21 & 0,09 & $-0,16$ & 0,04 & 0,35 & 0,01 & 0,20 & 0,38 \\
\hline miR-15a-5p & B & $-0,22$ & 0,19 & 1,04 & 0,00 & $-1,32$ & 0,00 & 0,09 & 0,05 & $-0,09$ & 0,60 \\
\hline miR-15b-5p & C & $-0,31$ & 0,05 & 1,10 & 0,00 & $-1,25$ & 0,00 & 0,22 & 0,00 & 0,29 & 0,00 \\
\hline miR-16-1-3p & B & $-0,22$ & 0,07 & 0,57 & 0,00 & 0,03 & 0,67 & 0,22 & 0,00 & 0,05 & 0,72 \\
\hline miR-16-2-3p & B & 0,13 & 0,22 & 0,80 & 0,00 & 0,11 & 0,07 & 0,18 & 0,11 & 0,08 & 0,49 \\
\hline miR-16-5p & B & $-0,46$ & 0,08 & 1,54 & 0,00 & $-1,38$ & 0,00 & 0,26 & 0,09 & 0,04 & 0,76 \\
\hline miR-17-5p & B & 0,08 & 0,49 & 1,97 & 0,00 & $-0,25$ & 0,01 & 0,17 & 0,06 & $-0,16$ & 0,28 \\
\hline miR-181a-5p & $A, B$ & $-0,22$ & 0,20 & 1,39 & 0,00 & $-0,40$ & 0,00 & 0,33 & 0,01 & 0,09 & 0,62 \\
\hline miR-181b-5p & $A, B$ & $-0,27$ & 0,01 & 0,91 & 0,00 & $-0,12$ & 0,03 & 0,28 & 0,01 & 0,18 & 0,17 \\
\hline miR-181c-5p & A & $-0,23$ & 0,16 & 1,86 & 0,00 & $-0,64$ & 0,00 & 0,27 & 0,02 & 0,13 & 0,41 \\
\hline miR-185-5p & $A, C$ & 0,00 & 0,97 & 1,28 & 0,00 & $-0,47$ & 0,00 & 0,04 & 0,55 & $-0,17$ & 0,27 \\
\hline miR-188-5p & $A$ & 0,16 & 0,00 & $-0,17$ & 0,88 & $-0,16$ & 0,00 & 0,01 & 0,68 & $-0,07$ & 0,61 \\
\hline miR-18a-5p & A & $-0,09$ & 0,35 & $-0,35$ & 0,30 & $-0,09$ & 0,20 & 0,28 & 0,00 & 0,20 & 0,17 \\
\hline miR-190b & $\mathrm{E}$ & 0,01 & 0,85 & $-0,73$ & 0,02 & 0,24 & 0,00 & 0,25 & 0,00 & 0,14 & 0,18 \\
\hline miR-192-5p & B & $-0,11$ & 0,43 & 0,78 & 0,00 & $-0,41$ & 0,00 & 0,12 & 0,32 & $-0,06$ & 0,79 \\
\hline miR-193a-3p & A & 0,13 & 0,37 & 0,87 & 0,00 & $-0,45$ & 0,00 & 0,20 & 0,01 & $-0,02$ & 0,95 \\
\hline miR-194-5p & $A$ & $-0,09$ & 0,56 & 0,44 & 0,01 & $-0,30$ & 0,00 & 0,16 & 0,04 & 0,04 & 0,64 \\
\hline miR-195-3p & $B, A$ & 0,06 & 0,36 & 0,68 & 0,00 & 0,39 & 0,00 & 0,37 & 0,01 & 0,25 & 0,32 \\
\hline miR-195-5p & $B, A, C$ & $-0,16$ & 0,22 & 1,64 & 0,00 & $-1,37$ & 0,00 & 0,20 & 0,07 & $-0,02$ & 0,98 \\
\hline miR-199a-3p & C & $-0,08$ & 0,55 & 0,13 & 0,27 & 0,09 & 0,13 & 0,30 & 0,00 & 0,21 & 0,02 \\
\hline miR-199a-5p & C & $-0,03$ & 0,68 & $-2,11$ & 0,00 & $-0,12$ & 0,09 & 0,25 & 0,02 & 0,18 & 0,22 \\
\hline$m i R-199 b-5 p$ & A & $-0,24$ & 0,01 & $-1,37$ & 0,00 & $-0,05$ & 0,25 & 0,24 & 0,02 & 0,02 & 0,75 \\
\hline miR-19a-3p & A & 0,29 & 0,01 & 0,82 & 0,00 & 0,57 & 0,00 & 0,25 & 0,01 & 0,17 & 0,23 \\
\hline miR-19b-3p & B & 0,11 & 0,19 & 0,12 & 0,25 & 0,53 & 0,00 & 0,32 & 0,01 & 0,35 & 0,10 \\
\hline miR-200c-3p & $E, C$ & 0,44 & 0,00 & 0,88 & 0,00 & $-0,18$ & 0,00 & 0,38 & 0,00 & 0,37 & 0,13 \\
\hline$m i R-200 c-5 p$ & $\mathrm{E}$ & $-0,29$ & 0,13 & 0,08 & 0,33 & $-0,20$ & 0,00 & 0,39 & 0,01 & 0,15 & 0,15 \\
\hline miR-204-5p & $A, D$ & 0,01 & 0,92 & 0,09 & 0,42 & 0,22 & 0,00 & 0,25 & 0,03 & 0,19 & 0,13 \\
\hline miR-208a-3p & A & $-0,10$ & 0,51 & $-0,60$ & 0,03 & $-0,13$ & 0,02 & 0,19 & 0,04 & 0,93 & 0,42 \\
\hline miR-208a-5p & A & 0,05 & 0,34 & 1,07 & 0,00 & $-0,33$ & 0,00 & $-0,03$ & 0,14 & $-0,11$ & 0,36 \\
\hline miR-208b-3p & C & $-0,11$ & 0,28 & $-0,08$ & 0,87 & $-0,27$ & 0,00 & 0,19 & 0,07 & 0,24 & 0,07 \\
\hline miR-20a-3p & C & $-0,34$ & 0,01 & 0,37 & 0,03 & $-0,35$ & 0,00 & 0,07 & 0,07 & $-0,14$ & 0,40 \\
\hline miR-20a-5p & C & $-0,12$ & 0,42 & 0,41 & 0,01 & $-0,07$ & 0,17 & 0,05 & 0,22 & $-0,18$ & 0,28 \\
\hline$m i R-20 b-5 p$ & $A$ & 0,22 & 0,37 & 0,77 & 0,00 & $-0,08$ & 0,25 & 0,27 & 0,00 & 0,19 & 0,21 \\
\hline miR-21-3p & $B, A$ & $-0,05$ & 0,66 & $-0,85$ & 0,00 & $-0,18$ & 0,00 & 0,04 & 0,59 & 0,22 & 0,02 \\
\hline miR-21-5p & $B, A, C$ & 0,17 & 0,05 & 0,10 & 0,25 & $-0,13$ & 0,05 & 0,11 & 0,25 & $-0,03$ & 0,93 \\
\hline miR-210-3p & A & 0,28 & 0,02 & 0,58 & 0,00 & 0,01 & 0,79 & 0,27 & 0,01 & 0,45 & 0,01 \\
\hline miR-210-5p & A & 0,00 & 0,82 & 0,64 & 0,00 & $-0,12$ & 0,02 & $-0,03$ & 0,64 & $-0,04$ & 0,80 \\
\hline miR-212-3p & $A, D$ & 0,06 & 0,36 & 0,22 & 0,11 & $-0,12$ & 0,00 & 0,22 & 0,08 & 0,36 & 0,12 \\
\hline$m i R-212-5 p$ & $A, D$ & $-0,38$ & 0,00 & 0,78 & 0,00 & $-0,61$ & 0,00 & 0,28 & 0,02 & 0,35 & 0,18 \\
\hline miR-214-3p & C & $-0,49$ & 0,00 & 0,59 & 0,00 & $-0,16$ & 0,00 & 0,05 & 0,06 & 0,14 & 0,22 \\
\hline miR-214-5p & C & 0,06 & 0,49 & $-0,22$ & 0,78 & $-0,07$ & 0,19 & $-0,04$ & 0,16 & $-0,13$ & 0,39 \\
\hline miR-216a-5p & D & 0,06 & 0,59 & $-1,60$ & 0,00 & $-0,06$ & 0,20 & 0,25 & 0,00 & 0,42 & 0,14 \\
\hline$m i R-218-5 p$ & C & 0,08 & 0,37 & 0,34 & 0,04 & $-0,08$ & 0,27 & 0,38 & 0,00 & 0,38 & 0,12 \\
\hline miR-22-3p & $B, A$ & $-0,04$ & 0,71 & $-1,28$ & 0,00 & $-0,32$ & 0,00 & 0,07 & 0,11 & $-0,14$ & 0,41 \\
\hline miR-22-5p & $B, A$ & $-0,20$ & 0,07 & 0,67 & 0,00 & $-0,48$ & 0,00 & 0,00 & 0,95 & $-0,07$ & 0,67 \\
\hline miR-221-3p & $B, C$ & $-0,04$ & 0,70 & 0,53 & 0,00 & 0,04 & 0,31 & 0,15 & 0,18 & $-0,04$ & 0,88 \\
\hline miR-221-5p & B & $-0,29$ & 0,00 & 0,41 & 0,01 & $-0,49$ & 0,00 & 0,23 & 0,07 & 0,20 & 0,27 \\
\hline miR-222-3p & $B, A, C$ & $-0,06$ & 0,45 & $-0,28$ & 0,42 & 0,05 & 0,27 & 0,18 & 0,07 & $-0,13$ & 0,23 \\
\hline$m i R-222-5 p$ & $B, A$ & 0,02 & 0,80 & $-0,40$ & 0,24 & $-0,30$ & 0,01 & 0,28 & 0,02 & 0,19 & 0,18 \\
\hline miR-223-3p & $B, A$ & $-0,24$ & 0,00 & $-0,17$ & 0,83 & 0,16 & 0,00 & 0,28 & 0,00 & 0,05 & 0,64 \\
\hline miR-223-5p & $B, A, C$ & $-0,43$ & 0,05 & 0,40 & 0,07 & $-0,43$ & 0,00 & 0,13 & 0,00 & 0,11 & 0,33 \\
\hline miR-23a-3p & $A, B, C$ & 0,07 & 0,08 & 1,15 & 0,00 & 0,32 & 0,00 & $-0,06$ & 0,36 & $-0,12$ & 0,20 \\
\hline miR-23a-5p & $A, B$ & $-0,02$ & 0,90 & $-0,57$ & 0,04 & 0,06 & 0,36 & 0,27 & 0,01 & 0,17 & 0,47 \\
\hline miR-23b-3p & $A, B$ & $-0,08$ & 0,19 & 1,09 & 0,00 & 0,38 & 0,00 & $-0,03$ & 0,70 & $-0,18$ & 0,30 \\
\hline miR-23b-5p & A, B & 0,19 & 0,18 & 0,43 & 0,01 & $-0,83$ & 0,00 & $-0,06$ & 0,31 & $-0,19$ & 0,27 \\
\hline miR-24-1-5p & $A$ & $-0,28$ & 0,05 & $-1,03$ & 0,00 & $-0,23$ & 0,00 & 0,09 & 0,30 & 0,02 & 0,76 \\
\hline miR-24-2-5p & $A, B$ & $-0,19$ & 0,26 & $-1,26$ & 0,00 & $-0,16$ & 0,00 & 0,34 & 0,01 & 0,66 & 0,20 \\
\hline miR-24-3p & A & 0,09 & 0,17 & 0,89 & 0,00 & 0,04 & 0,40 & 0,05 & 0,42 & 0,00 & 0,88 \\
\hline miR-26a-5p & A & 0,01 & 0,90 & 1,41 & 0,00 & 0,01 & 0,86 & 0,09 & 0,29 & 0,08 & 0,58 \\
\hline$m i R-26 b-5 p$ & A & 0,07 & 0,58 & 1,18 & 0,00 & 0,09 & 0,07 & 0,13 & 0,24 & 0,05 & 0,67 \\
\hline miR-27a-3p & A & 0,01 & 0,93 & 0,19 & 0,12 & 0,27 & 0,00 & 0,20 & 0,06 & 0,20 & 0,36 \\
\hline
\end{tabular}




\begin{tabular}{|c|c|c|c|c|c|c|c|c|c|c|c|}
\hline $\operatorname{miR}-27 b-3 p$ & $A, C$ & $-0,19$ & 0,04 & $-0,15$ & 0,91 & 0,28 & 0,00 & 0,04 & 0,61 & 0,04 & 0,61 \\
\hline miR-29a-3p & A & 0,20 & 0,01 & $-2,30$ & 0,00 & $-0,27$ & 0,00 & 0,08 & 0,03 & 0,06 & 0,64 \\
\hline miR-29b-3p & A & 0,07 & 0,50 & $-2,45$ & 0,00 & $-0,20$ & 0,00 & 0,21 & 0,09 & $-0,10$ & 0,44 \\
\hline $\operatorname{miR}-29 c-3 p$ & A & 0,00 & 0,95 & $-2,23$ & 0,00 & $-0,16$ & 0,00 & 0,10 & 0,34 & $-0,15$ & 0,27 \\
\hline miR-29c-5p & A & 0,07 & 0,33 & 0,46 & 0,01 & $-0,16$ & 0,04 & $-0,25$ & 0,34 & $-0,03$ & 0,96 \\
\hline miR-302a-3p & E & $-0,15$ & 0,25 & 0,68 & 0,00 & 0,01 & 0,89 & 0,18 & 0,00 & $-0,22$ & 0,19 \\
\hline miR-302a-5p & E & 0,07 & 0,52 & 0,71 & 0,00 & $-0,02$ & 0,70 & 0,09 & 0,17 & 0,17 & 0,14 \\
\hline $\operatorname{miR}-302 c-3 p$ & E & $-0,02$ & 0,85 & 0,61 & 0,00 & $-0,03$ & 0,67 & 0,06 & 0,11 & $-0,19$ & 0,23 \\
\hline miR-302d-5p & D & 0,22 & 0,01 & 1,07 & 0,00 & $-0,23$ & 0,01 & $-0,03$ & 0,68 & $-0,13$ & 0,37 \\
\hline miR-30a-3p & A & $-0,08$ & 0,44 & 0,82 & 0,00 & $-0,12$ & 0,07 & 0,38 & 0,00 & 0,89 & 0,12 \\
\hline miR-30a-5p & A & 0,04 & 0,75 & 1,50 & 0,00 & $-0,12$ & 0,00 & 0,07 & 0,10 & 0,06 & 0,41 \\
\hline miR-30b-3p & A & $-0,13$ & 0,44 & 1,38 & 0,00 & $-0,40$ & 0,00 & 0,29 & 0,00 & 0,11 & 0,40 \\
\hline miR-30b-5p & A & 0,14 & 0,25 & 1,31 & 0,00 & $-0,13$ & 0,02 & 0,05 & 0,12 & 0,09 & 0,29 \\
\hline miR-30c-2-3p & A & $-0,13$ & 0,55 & 0,85 & 0,00 & $-0,27$ & 0,01 & $-0,17$ & 0,06 & $-0,33$ & 0,01 \\
\hline$m i R-30 c-5 p$ & A & $-0,01$ & 0,99 & 1,16 & 0,00 & $-0,16$ & 0,01 & 0,01 & 0,88 & 0,10 & 0,35 \\
\hline miR-30d-5p & A & $-0,08$ & 0,68 & 1,41 & 0,00 & 0,05 & 0,20 & 0,05 & 0,11 & 0,13 & 0,28 \\
\hline miR-30e-3p & $A, B$ & 0,30 & 0,06 & 0,70 & 0,00 & $-0,05$ & 0,46 & 0,28 & 0,01 & 0,41 & 0,08 \\
\hline$m i R-30 e-5 p$ & $A, B, C$ & $-0,04$ & 0,79 & 1,31 & 0,00 & $-0,26$ & 0,00 & 0,06 & 0,17 & 0,03 & 0,73 \\
\hline miR-320a & B & 0,19 & 0,09 & 0,34 & 0,05 & $-0,24$ & 0,02 & 0,03 & 0,54 & $-0,10$ & 0,45 \\
\hline miR-331-3p & A & $-0,14$ & 0,12 & 1,52 & 0,00 & $-0,12$ & 0,15 & $-0,12$ & 0,28 & $-0,30$ & 0,07 \\
\hline miR-335-5p & A & 0,17 & 0,02 & 0,51 & 0,03 & $-0,27$ & 0,00 & 0,25 & 0,07 & 0,23 & 0,08 \\
\hline miR-338-3p & A & $-0,34$ & 0,01 & 1,37 & 0,00 & $-0,41$ & 0,00 & 0,16 & 0,20 & 0,16 & 0,26 \\
\hline miR-340-5p & $A$ & $-0,14$ & 0,06 & 0,20 & 0,25 & $-0,38$ & 0,00 & 0,23 & 0,03 & 0,05 & 0,73 \\
\hline miR-342-3p & A & $-0,12$ & 0,20 & 0,10 & 0,28 & 0,17 & 0,02 & 0,26 & 0,02 & 0,29 & 0,20 \\
\hline miR-345-5p & $A$ & 0,10 & 0,36 & 0,43 & 0,08 & $-0,18$ & 0,01 & 0,09 & 0,00 & $-0,18$ & 0,10 \\
\hline miR-34a-3p & $A, B$ & $-0,23$ & 0,02 & 0,71 & 0,00 & $-0,61$ & 0,00 & $-0,32$ & 0,01 & $-0,22$ & 0,03 \\
\hline$m i R-34 a-5 p$ & $A, B$ & 0,00 & 0,89 & 0,79 & 0,00 & $-0,42$ & 0,00 & 0,19 & 0,00 & 0,07 & 0,62 \\
\hline miR-361-3p & $B, A$ & 0,08 & 0,04 & 1,02 & 0,00 & 0,11 & 0,06 & 0,07 & 0,16 & $-0,13$ & 0,36 \\
\hline miR-361-5p & $B, A$ & $-0,45$ & 0,00 & $-0,36$ & 0,24 & $-0,24$ & 0,00 & 0,17 & 0,04 & 0,18 & 0,03 \\
\hline miR-378a-3p & $B, A, C$ & 0,01 & 0,88 & 1,12 & 0,00 & $-0,45$ & 0,00 & $-0,01$ & 0,86 & 0,01 & 0,83 \\
\hline miR-378a-5p & $B, A$ & $-0,15$ & 0,21 & $-0,31$ & 0,43 & $-0,45$ & 0,00 & 0,19 & 0,00 & 0,00 & 0,92 \\
\hline miR-379-3p & A & 0,00 & 0,96 & 1,62 & 0,00 & 0,02 & 0,64 & 0,16 & 0,02 & $-0,19$ & 0,25 \\
\hline miR-379-5p & A & $-0,03$ & 0,80 & 0,25 & 0,07 & $-0,66$ & 0,00 & 0,11 & 0,06 & $-0,09$ & 0,54 \\
\hline$m i R-409-5 p$ & D & $-0,15$ & 0,42 & $-0,55$ & 0,09 & $-0,37$ & 0,00 & 0,01 & 0,64 & 0,08 & 0,29 \\
\hline miR-410-3p & E & 0,10 & 0,58 & 0,29 & 0,05 & $-0,16$ & 0,00 & 0,28 & 0,00 & 0,16 & 0,32 \\
\hline miR-422a & E & $-0,09$ & 0,61 & $-0,50$ & 0,08 & $-0,60$ & 0,00 & 0,07 & 0,43 & $-0,05$ & 0,76 \\
\hline$m i R-423-5 p$ & $\mathrm{E}$ & $-0,14$ & 0,32 & 0,07 & 0,31 & $-0,22$ & 0,01 & 0,16 & 0,01 & 0,17 & 0,07 \\
\hline$m i R-425-3 p$ & B & $-0,23$ & 0,05 & $-0,13$ & 0,99 & $-0,16$ & 0,02 & 0,08 & 0,47 & $-0,11$ & 0,48 \\
\hline$m i R-425-5 p$ & B & 0,04 & 0,24 & 1,11 & 0,00 & 0,16 & 0,00 & 0,14 & 0,06 & 0,08 & 0,55 \\
\hline miR-431-5p & E & 0,32 & 0,00 & 1,43 & 0,00 & $-0,39$ & 0,00 & $-0,05$ & 0,10 & $-0,22$ & 0,24 \\
\hline miR-432-5p & D & 0,03 & 0,54 & 0,09 & 0,31 & $-0,35$ & 0,00 & 0,20 & 0,01 & 0,04 & 0,61 \\
\hline$m i R-450 b-3 p$ & D & 0,00 & 0,96 & 0,16 & 0,20 & $-0,44$ & 0,00 & $-0,03$ & 0,71 & $-0,15$ & 0,13 \\
\hline miR-466 & A & $-0,01$ & 0,98 & $-0,46$ & 0,12 & 0,08 & 0,29 & 0,33 & 0,00 & 0,16 & 0,28 \\
\hline miR-484 & $A, C$ & 0,06 & 0,26 & $-0,04$ & 0,66 & $-0,02$ & 0,81 & $-0,24$ & 0,06 & $-0,23$ & 0,15 \\
\hline$m i R-486-3 p$ & $B, A$ & $-0,54$ & 0,00 & 0,09 & 0,27 & $-0,26$ & 0,00 & 0,21 & 0,03 & $-0,03$ & 0,92 \\
\hline$m i R-486-5 p$ & $B, A, C$ & 0,00 & 0,94 & 0,64 & 0,00 & $-0,22$ & 0,00 & $-0,04$ & 0,60 & $-0,10$ & 0,48 \\
\hline$m i R-488-5 p$ & $\mathrm{D}$ & 0,10 & 0,29 & $-1,03$ & 0,00 & $-0,41$ & 0,00 & $-0,34$ & 0,00 & $-0,18$ & 0,13 \\
\hline$m i R-494-3 p$ & A & 0,04 & 0,64 & $-0,03$ & 0,64 & $-0,11$ & 0,10 & 0,21 & 0,01 & $-0,09$ & 0,47 \\
\hline miR-497-5p & A & $-0,44$ & 0,03 & 2,32 & 0,00 & $-1,70$ & 0,00 & 0,08 & 0,02 & $-0,04$ & 0,77 \\
\hline miR-499a-5p & A & $-0,17$ & 0,03 & $-0,72$ & 0,01 & $-0,16$ & 0,00 & 0,36 & 0,00 & 0,14 & 0,10 \\
\hline miR-511-3p & D & $-0,02$ & 0,90 & 1,09 & 0,00 & 0,05 & 0,45 & 0,08 & 0,14 & $-0,11$ & 0,47 \\
\hline miR-511-5p & D & 0,02 & 0,41 & 1,03 & 0,00 & $-0,11$ & 0,05 & 0,14 & 0,05 & 0,31 & 0,15 \\
\hline miR-520f-3p & E & 0,03 & 0,78 & 0,84 & 0,00 & 0,35 & 0,01 & 0,07 & 0,17 & $-0,18$ & 0,19 \\
\hline miR-520f-5p & $\mathrm{E}$ & 0,16 & 0,08 & $-0,05$ & 0,70 & 0,00 & 0,93 & $-0,05$ & 0,18 & $-0,13$ & 0,27 \\
\hline miR-532-5p & A & $-0,14$ & 0,22 & $-1,07$ & 0,00 & $-0,29$ & 0,00 & 0,16 & 0,03 & 0,05 & 0,67 \\
\hline miR-558 & D & $-0,05$ & 0,52 & 0,05 & 0,37 & $-0,18$ & 0,01 & 0,13 & 0,00 & $-0,06$ & 0,62 \\
\hline miR-582-5p & A & $-0,19$ & 0,08 & $-0,06$ & 0,75 & $-0,20$ & 0,00 & 0,26 & 0,00 & 0,30 & 0,00 \\
\hline miR-590-5p & C & 0,17 & 0,00 & 0,20 & 0,12 & $-0,19$ & 0,00 & 0,33 & 0,01 & 0,39 & 0,01 \\
\hline miR-629-5p & D & $-0,05$ & 0,55 & 1,04 & 0,00 & $-0,16$ & 0,02 & 0,07 & 0,34 & $-0,26$ & 0,10 \\
\hline miR-631 & D & $-0,31$ & 0,01 & 1,65 & 0,00 & $-0,45$ & 0,00 & $-0,09$ & 0,11 & $-0,16$ & 0,11 \\
\hline$m i R-7-5 p$ & A & $-0,38$ & 0,01 & 3,09 & 0,00 & $-0,42$ & 0,00 & 0,04 & 0,71 & 0,04 & 0,79 \\
\hline miR-877-5p & A & $-0,30$ & 0,03 & 1,22 & 0,00 & $-0,15$ & 0,11 & 0,01 & 0,95 & 0,09 & 0,65 \\
\hline miR-889-3p & D & $-0,13$ & 0,20 & 1,03 & 0,00 & 0,10 & 0,10 & 0,28 & 0,00 & 0,16 & 0,26 \\
\hline miR-889-5p & D & $-0,07$ & 0,61 & 1,04 & 0,00 & $-0,79$ & 0,05 & 0,00 & 0,93 & $-0,25$ & 0,08 \\
\hline miR-891a-3p & D & $-0,48$ & 0,00 & $-1,43$ & 0,00 & $-0,51$ & 0,00 & $-0,28$ & 0,00 & 0,05 & 0,67 \\
\hline miR-891a-5p & D & $-0,07$ & 0,50 & 0,35 & 0,08 & $-0,11$ & 0,01 & $-0,04$ & 0,45 & 0,04 & 0,76 \\
\hline $\mathrm{miR}-892 \mathrm{~b}$ & D & $-0,21$ & 0,03 & 0,26 & 0,10 & $-0,34$ & 0,00 & 0,02 & 0,60 & $-0,10$ & 0,42 \\
\hline
\end{tabular}




\begin{tabular}{l|l|ll|llll|llll} 
miR-9-5p & A & $-0,40$ & 0,02 & 1,34 & 0,00 & 0,15 & 0,02 & 0,25 & 0,02 & 0,05 & 0,68 \\
miR-92b-3p & D & 0,34 & 0,00 & $-0,31$ & 0,48 & 0,08 & 0,14 & $-0,15$ & 0,18 & $-0,10$ & 0,49 \\
miR-96-5p & A & $-0,44$ & 0,09 & 0,48 & 0,03 & $-0,39$ & 0,00 & 0,12 & 0,11 & 0,33 & 0,02 \\
miR-98-5p & A & $-0,13$ & 0,09 & 0,82 & 0,00 & $-0,10$ & 0,07 & 0,23 & 0,18 & 0,38 & 0,46 \\
miR-99a-5p & A & $-0,17$ & 0,00 & $-0,23$ & 0,60 & 0,02 & 0,61 & 0,02 & 0,78 & $-0,06$ & 0,60 \\
miR-99b-5p & A, C & $-0,17$ & 0,01 & $-0,13$ & 0,96 & 0,04 & 0,49 & 0,02 & 0,77 & $-0,16$ & 0,32
\end{tabular}




\section{Chapter 5}

\section{Literature-based gene to phenotype interaction network analysis reveals novel microRNA regulators in heart failure}

\section{Authors:}

Wouter Derks ${ }^{1 *}$

Georg Summer ${ }^{1,2^{*}}$

Rob Coenen ${ }^{1}$

Stephane Heymans ${ }^{1}$

Marc van Bilsen ${ }^{1,3}$

1.) Department of Cardiology, Cardiovascular Research Institute Maastricht (CARIM), Maastricht University, Maastricht, the Netherlands.

2.) TNO, Microbiology \& Systems Biology, Zeist, the Netherlands.

3.) Department of Physiology, Cardiovascular Research Institute Maastricht (CARIM), Maastricht University, Maastricht, the Netherlands.

*WD and GS Contributed equally

\section{Submitted}




\begin{abstract}
Chronic hypertension and aortic stenosis often lead to pressure overload-induced heart failure (HF). This is associated with maladaptive structural changes, marked by the enlargement of cardiomyocytes (hypertrophy) and excessive collagen deposition (fibrosis). More recently, the role of cardiac inflammation in the aetiology of HF is rapidly gaining attention. It remains to be further established, however, if and how inflammation, hypertrophy and fibrosis influence each other. In cardiac research genetically manipulated mouse models are crucial to study the molecular mechanisms underlying HF. In this study we extracted data from 284 preclinical articles on pressure overload-induced HF published between 2000 and 2014. This unique dataset was subsequently used (1) to investigate the relationship between inflammation, hypertrophy and fibrosis, (2) to generate a gene to phenotype interaction network (GPIN) allowing the identification of critical molecular players in HF development, and (3) to compare the features and define reference values of phenotypical outcome measures of the most commonly used models of pressure overload. By applying a meta-analysis based approach on preclinical studies we demonstrate that during HF development the process of cardiac inflammation is intricately linked with hypertrophy and fibrosis. Furthermore, by extending the dataset-derived GPIN with publically available microRNA targeting data we were able to identify various microRNAs (among which miR-466l, miR-124-3p and miR-30e-5p) predicted to have a large impact on cardiac inflammation, hypertrophy or fibrosis. In conclusion, we present a novel methodology to build and mine a gene-to-phenotype interaction network. This approach enabled us to generate new knowledge based on previously published cardiovascular research.
\end{abstract}




\section{Introduction}

Persistent hypertension and aortic stenosis are major risk factors for the development of heart failure (HF) ${ }^{1}$. Both conditions lead to pressure overload (PO) in the heart and initiate maladaptive cardiac remodelling characterized by enlargement of cardiomyocytes (hypertrophy) ${ }^{2}$ and increased collagen deposition (fibrosis) ${ }^{3}$. More recently, inflammation is recognized as an important player in the development of $\mathrm{PO}$-induced $\mathrm{HF}{ }^{4,5}$. Via the infiltration of leukocytes, the activation of resident immune cells, or the activation of proinflammatory signalling pathways within cardiomyocytes, inflammation has been shown to influence the development of hypertrophy and fibrosis, and consequently HF progression ${ }^{6,7}$.

Although recognized as a highly valuable tool in clinical science for decades, the application of meta-analyses and systematic reviews to preclinical studies has started only recently ${ }^{8-10}$. In preclinical cardiac research, rodent models of PO are used routinely to investigate the molecular mechanisms involved in the pathogenesis of non-ischemic HF. Rodent models have been developed in which cardiac PO is being imposed either via surgical intervention (Aortic Constriction, AC) or by pharmacological intervention (Angiotensin II or Isoproterenol infusion). Accordingly, in the present study we performed a systematic literature search for $\mathrm{PO}$-induced $\mathrm{HF}$ in rats and mice. Experimental details and outcome parameters (OCPs) were extracted from almost 300 articles and used to explore the relation between inflammation, on the one hand, and hypertrophy and fibrosis on the other.

Genetically modified mouse models are extensively used to investigate the molecular mechanisms involved in the pathogenesis of PO induced heart failure ${ }^{11}$. As the genetic manipulation (gain- or loss-of-function) of specific genes implicated in hypertrophy, fibrosis or inflammation forms the core of many studies, this information was used to build a gene to phenotype interaction network (GPIN) from the screened articles. The created network visualizes the effect these specific genes have on the HF processes resembled by the OCPs. Next, as microRNAs (miRNAs) are established negative regulators of gene-expression and have been shown to regulate pathophysiological processes associated with $\mathrm{HF}^{12-14}$, we extended the GPIN with miRNA targeting data. Based on the number of genes in the network that these miRNAs are predicted to target a score was calculated to indicate their relevance. This allows the identification of candidate miRNAs influencing cardiac inflammation, hypertrophy and fibrosis.

In addition, this extensive dataset was used to compare features of frequently used rodent PO models of which, despite their widespread use, the similarities and dissimilarities are ill-defined and reference values for the expected degree of hypertrophy, fibrosis and inflammation are still lacking. This comparison aids the interpretation of performed studies and the design of future PO studies.

In summary, here we present an approach for using existing literature to identify potential novel HF therapeutic targets by creating and mining a Gene to Phenotype interaction network based on a systematic review. Secondly, we explore the relation between inflammation, on the one hand, and hypertrophy and fibrosis on the other. A final goal was to define reference values for pathophysiological parameters, such as hypertrophy, fibrosis and inflammation in different models of hypertensive heart disease. 


\section{Methods}

\section{Search strategy}

Inclusion and exclusion criteria are listed in Table 1. In short, for inclusion a rat or mouse model of cardiac PO had to be used and a numerical quantification of at least 2 of the 3 outcome parameters (OCPs, being hypertrophy, fibrosis or inflammation) had to be given. A wild type control group or sham group also had to be part of the study design. Reviews, editorials and other non-experimental articles were excluded as well as articles in a language other than English. Based on these criteria a search strategy was formulated consisting of different components: disease model, species, selection of at least 2 of the 3 OCPs, organ, and additional filters for language and publication date. The combination of MeSH terms used to conduct the search is provided in the supplemental methods. Subsequently the developed search strategy was used on PubMed in March 2015, covered the years 2000 2014 and yielded 581 search results.

Table 1. Inclusion and exclusion criteria

\begin{tabular}{|c|c|}
\hline Inclusion criteria & Exclusion criteria \\
\hline PO inducing intervention & Full text in language other than English \\
\hline Rat or mouse studies & No original data (review, opinion etc.) \\
\hline \multirow{3}{*}{$\begin{array}{l}\text { Numerical outcome data for hypertrophy and fibrosis, } \\
\text { hypertrophy and inflammation fibrosis and } \\
\text { inflammation or all three }\end{array}$} & $\begin{array}{l}\text { Only addressing one of the OCPs of hypertrophy, fibrosis } \\
\text { and inflammation }\end{array}$ \\
\hline & No full text available \\
\hline & Lack of proper control groups \\
\hline
\end{tabular}

\section{Screening abstracts for inclusion}

The titles and abstracts of the retrieved articles were reviewed for selection by two authors (WD and GS). Full-text articles were retrieved from abstracts selected for further review. Abstracts of the 581 articles from the PubMed search were screened for relevance to the inclusion criteria (Table 1). Based on the abstract we excluded 231 articles (Figure 1). Based on the full text another 139 articles were excluded. By cross-checking the references of included articles, 73 additional papers were identified as relevant and included in the dataset. Some articles contained more than one study, for example, when TAC and Angll models were used in parallel. In that case, they were dealt with as separate studies, yielding an additional 85 studies, and taking the total amount of studies to 369 (Figure 1). 


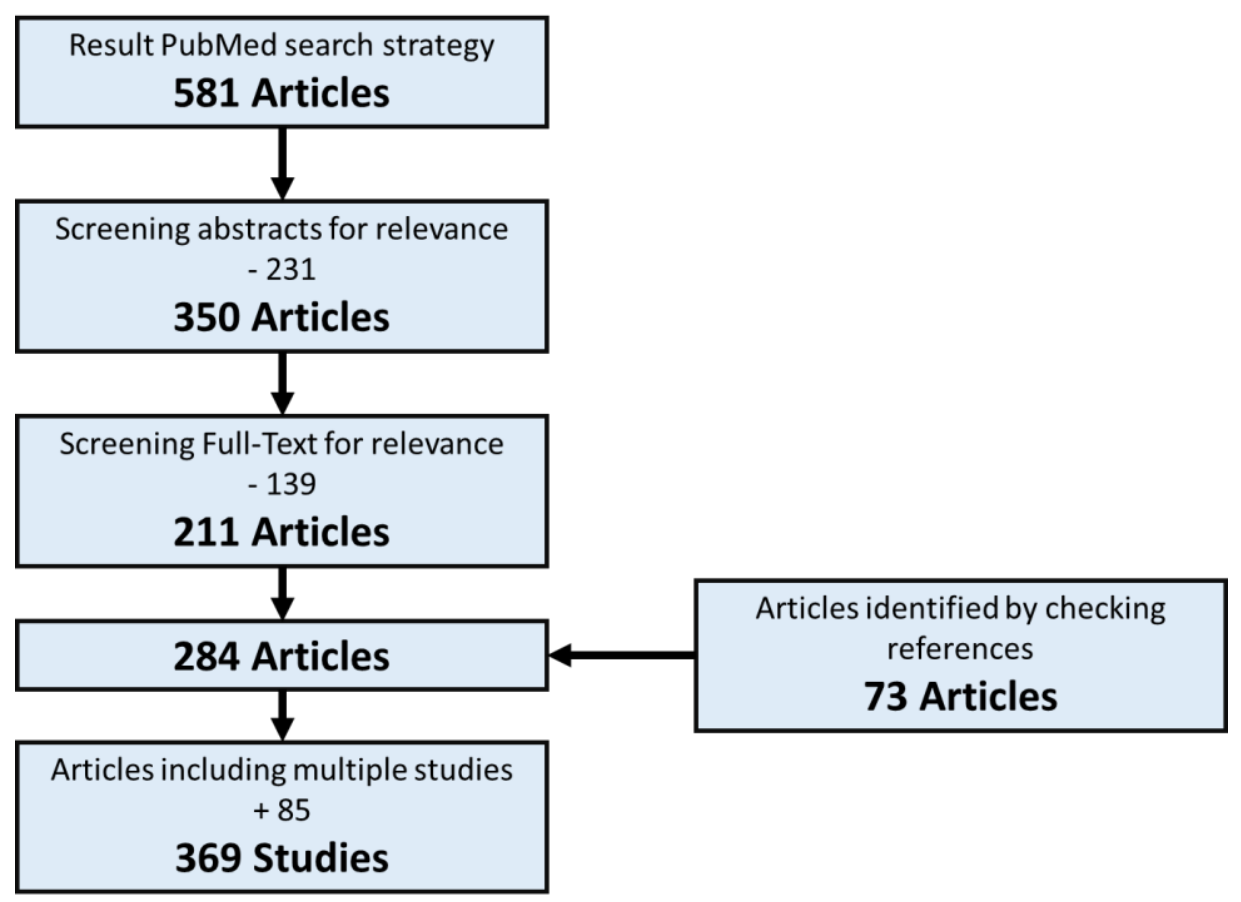

Figure 1. Flow chart of article selection procedure. Selection based on abstract and full text was performed by two authors. Additional 73 articles were added based on checking reference list in selected papers.

\section{Data extraction}

Experimental details and numerical data were extracted from the articles. In case data were presented in the form of figures, we used the freeware program DataThief III (B. Tummers, 2006, http://datathief.org/) to convert graphical information into numerical data. Supplemental Table 1 shows all the experimental details and OCPs extracted from the articles, comprising of: basal characteristics of the study the experimental details, OCPs and indications on how these OCPs were measured and presented. Where different measures of an OCP were reported we extracted the one most commonly used. From all studies we extracted mean values for 4 groups:

1. Control - Sham. Group that did not receive an experimental treatment and was not subjected to pressure overload (e.g. wild-type, sham-operated mice).

2. Experimental - Sham. Group that received the experimental treatment, but was not subjected to pressure overload (e.g. knockout, sham-operated mice).

3. Control-PO. Group that did not receive the experimental treatment, but was subjected to pressure overload (e.g. wild-type mice subjected to TAC).

4. Experimental - PO. Group that received the experimental treatment and was subjected to pressure overload (e.g. knockout mice subjected to TAC).

From the text of the paper we determined a general conclusion on how the experimental condition affected hypertrophy, fibrosis, cardiac function and inflammation and simplified the conclusions into: reduced $(\downarrow)$, increased $(\uparrow)$ or no significant change (-). These conclusions were not only based on numerical data. 


\section{Statistics and subgroup analysis}

Data in bar graphs and tables are presented as mean \pm SEM unless indicated otherwise. Statistical analyses were performed using GraphPad Prism 5. For multiple group comparisons a one-way ANOVA was performed. Comparisons between two groups were done using unpaired Student's t-tests. A two-tailed P-value of $<0.05$ was considered as statistically significant. Pearson's $r$ test was used to determine correlations in scatterplots. Fisher's exact test was used for comparison of categorical data.

\section{Gene to phenotype network creation}

We combined the effects of genetic manipulations with the effect of the PO model on the OCPs to create gene to phenotype interactions (GPIs). The effects are based on the comparison of the $\mathrm{Wt}-\mathrm{PO}$ and Experimental - PO group and on the overall conclusion of the study regarding the effect on OCPs. GPIs are directional (always from the gene towards the phenotype) and typed, that is, they describe the overall effect of the gene on an OCP. The type of GPI is either pro, anti or neutral. If the genetic manipulation in a study decreases the expression or function of a gene and the PO model leads to a reduction in the OCP, then the GPI is considered pro. For example, when mice with a knockout of the Tnf gene (representing a decrease in function of this gene) are subjected to TAC and subsequently cardiac fibrosis is significantly less than in wild-type mice subjected to TAC, than the GPI for Tnf and fibrosis is considered pro as the study implicates Tnf to be pro-fibrotic. The inverse case, overexpression of Tnf leading to an increase in fibrosis, is also a combination that leads to a pro typed GPI. Anti GPIs result from the genetic manipulation and the outcome opposing each other, e.g. a knockout of IL-10 resulting in an increase in fibrosis. The third type for a GPI is neutral: it represents the cases in which the genetic manipulations do not significantly affect a specific OCP. All GPIs are based on the individual studies and do not account for experimental differences between studies. This can lead to contradictory types for GPIs in which the majority type is used for the gene to phenotype interaction network (GPIN), but the confidence in a particular GPI is reduced to reflect the difference between studies. For instance, if three studies result in a pro type and one neutral, the confidence for the GPI is 0.75 (Figure 2). Table 2 summarizes the rules for typing GPIs. The collection of GPIs from all studies were combined into a single network.

Table 2. Rules for typing gene to phenotype interactions based on general conclusion.

\begin{tabular}{lll}
\hline Genetic manipulation of gene of interest & Effect on OCP (general conclusion) & GPI type \\
\hline down-regulation & Reduction $\downarrow$ & Pro \\
\hline up-regulation & Increase $\uparrow$ & \multirow{2}{*}{ Anti } \\
\hline down-regulation & Increase $\uparrow$ & neutral \\
\hline up-regulation & Reduction $\downarrow$ & no significant change
\end{tabular}

\section{Enriching the GPIN}

In the first step we merged miRNA targeting information into the GPIN. MiRTarbase $4.5^{15}$ served as a source for validated miRNA targeting information (9.199 interactions). For predicted miRNA interactions the following databases were used: TargetScan 6.2 (160.413 interactions) ${ }^{16}$, miRDB 5.0 (257.330 interactions) ${ }^{17}$ and DIANA microT-CDS (530.955 interactions) ${ }^{18}$. The miRNA targeting information is consolidated into a single interaction that is weighted based on how many data sources predict a particular interaction. 


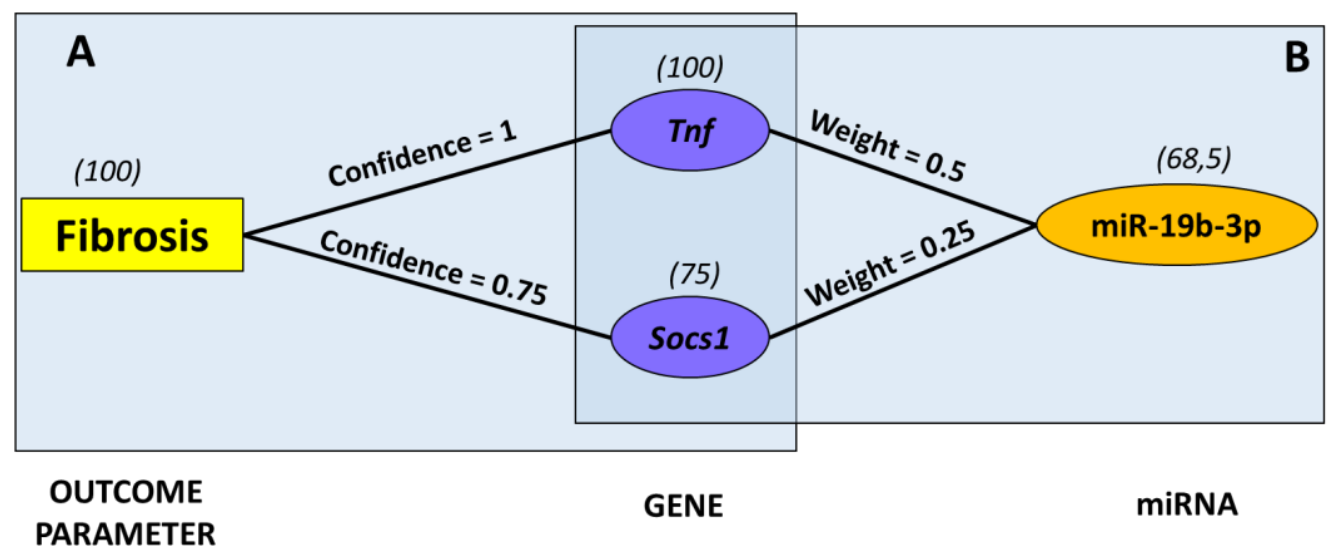

Figure 2. Workflow for scoring the relevance of miRNAs based on their targets and how those are connected to the OCPs. The score contribution from an OCP via the genes is depicted as the number between brackets (100). In box $A$, the score from the OCP is multiplied with the confidence of the GPI. Next the score of a gene is weighted based on the miRNA targeting information (box B). The sum of all scores flowing to a miRNA reflects its relevance for the OCP (in this example fibrosis). The schematic example in this figure is not based on the real data.

\section{Identifying heart failure relevant miRNAs}

The identification and prioritization of miRNAs relevant to HF is driven by a neighbourhood based network analysis strategy. Herewith, OCPs and genes are connected via GPIs and then via the miRNA targeting interactions to miRNAs. In the first step (Figure 2, box A) all genes connected to an OCP via a GPI are scored. For the gene score the confidence of a GPI is used. This leaves every gene connected with an OCP with one score for each of the OCPs. In the next step (Figure 2, box B) the genes confidence score is transferred to each microRNA targeting a particular gene. The genes confidence score is multiplied with the weight of a miRNA targeting interaction to calculate the contribution of a gene to the score of a miRNA. As one miRNA is often predicted to target multiple genes the transferred scores are accumulated to calculate the relevance scores of a miRNA for each of the OCPs. The final scores are then scaled between 0 and 1 and added to obtain the overall relevance score.

\section{Scripts and Software}

The software to build and enrich the GPIN are available at https://github.com/gsummer. The algorithm to score microRNA for their relevance regarding the OCPs can be found at https://github.com/gsummer/netlib-curator. In short, the GPIN was built iteratively by first adding relevant nodes (all genes from ENSEMBL 81 and mature miRNAs from miRBase 21) and then merging the different interaction databases into the GPIN. cyNeo4j ${ }^{19}$ was used to extract sub-networks from the GPIN into Cytoscape ${ }^{20}$ for visualization. 


\section{Results}

The selection of articles according to our pre-set criteria (Table 1, Figure 1) yielded a dataset of 369 pressure overload (PO) studies derived from 284 articles published between 2000 and 2014 (listed in supplementary file 1). Most studies (82\%) made use of mice, with the number of rat studies steadily decreasing over the years (from $60 \%$ in 2000 to $8 \%$ in 2014). By far the most commonly applied PO models in mice are aortic constriction (71\%) and angiotensin-II infusion (24\%, Table 3). As for the rat, more variation in the PO models applied was observed. The main characteristics of the most frequently applied PO models are summarized in the Supplemental Results and Supplementary Table 2.

The majority of studies made use of males (71.3\%) and only a limited number of studies used mixed gender or females. Notably, in $16 \%$ of the studies gender was not specified. The most commonly used strains were C57BL/6 for mice and Sprague-Dawley for rats (Table 3). Only 79 out of the 206 studies using C57BL/6 mice provided information about which substrain (6-J, 6-N) was used. The average age at the start of the experiment was 10.7 \pm 3.8 weeks for mice and $10.6 \pm 6.4$ weeks for rats and in most studies (31\%) the animals were subjected to 28 days of pressure overload.

Table 3. Overview of the study characteristics of the dataset.

\begin{tabular}{|c|c|c|c|c|c|}
\hline PO-intervention & $A C(n=243)$ & Angll ( $n=82)$ & ISO (n=12) & SHR $(n=15)$ & Other $(n=16)$ \\
\hline Mouse (n=306) & $71 \%$ & $24 \%$ & $3 \%$ & - & $2 \%$ \\
\hline Rat $(n=63)$ & $41 \%$ & $14 \%$ & $3 \%$ & $24 \%$ & $17 \%$ \\
\hline Total $(n=369)$ & $66 \%$ & $22 \%$ & $3 \%$ & $4 \%$ & $4 \%$ \\
\hline Gender & Male $(n=275)$ & Female $(n=19)$ & Both $(n=16)$ & ND $(n=60)$ & \\
\hline Mouse (n=306) & $71 \%$ & $5 \%$ & $5 \%$ & $19 \%$ & \\
\hline Rat $(n=63)$ & $89 \%$ & $5 \%$ & $5 \%$ & $2 \%$ & \\
\hline Total $(n=369)$ & $74 \%$ & $5 \%$ & $4 \%$ & $16 \%$ & \\
\hline Mouse strain & C57BL/6 $(n=206)$ & FVB $(n=20)$ & $C D-1(n=6)$ & Other $(n=40)$ & ND $(n=36)$ \\
\hline$(n=306)$ & $67 \%$ & $6 \%$ & $2 \%$ & $13 \%$ & $12 \%$ \\
\hline Rat strain & Wistar (n=13) & WKY (n=13) & Dahl $(n=5)$ & $S D(n=30)$ & Other $(n=2)$ \\
\hline$(n=63)$ & $21 \%$ & $21 \%$ & $8 \%$ & $49 \%$ & $1 \%$ \\
\hline
\end{tabular}

\section{Aortic constriction or Angiotensin II?}

The large number of mouse studies in the dataset (Table 3) provides the statistical power for a more detailed analysis of the phenotype induced by the two most commonly used PO interventions, i.e., trans-aortic constriction (TAC) and Angll infusion.

The effects of TAC and Angll on cardiac hypertrophy, fibrosis and function are shown in Figure 3. To exclude effects of differences in duration on outcome, only studies with a duration of 28 days were included in this analysis. The TAC model shows a more pronounced increase in cardiac mass $(+54.9 \%$ vs. $+31.1 \%, \mathrm{P}<0.01)$ and cardiomyocyte size $(+76.3 \%$ vs. $+62.1 \%$, N.S.) compared to Angll, indicative of a more pronounced hypertrophic response induced by TAC (Figure $3 A-B$ ). As for fractional shortening and collagen area, the dataset reveals no pronounced differences between these two PO models (Figure 3C-D). In 
conclusion, this analysis shows that TAC induces more pronounced cardiac hypertrophy compared to Angll infusion in mice, but this is not associated with a concurrent increase in fibrosis, or a more pronounced decline in systolic function.

In the Supplemental Results section of this manuscript an extensive description of PO models is given, including reference values of cardiac phenotypes induced by different PO interventions (Supplemental Table 2). In addition, an overview of the temporal changes induced after PO in mice (Supplemental Figure 1) as well as dose related effects on blood pressure and the PO induced phenotype (Supplemental Figure 2) are provided.
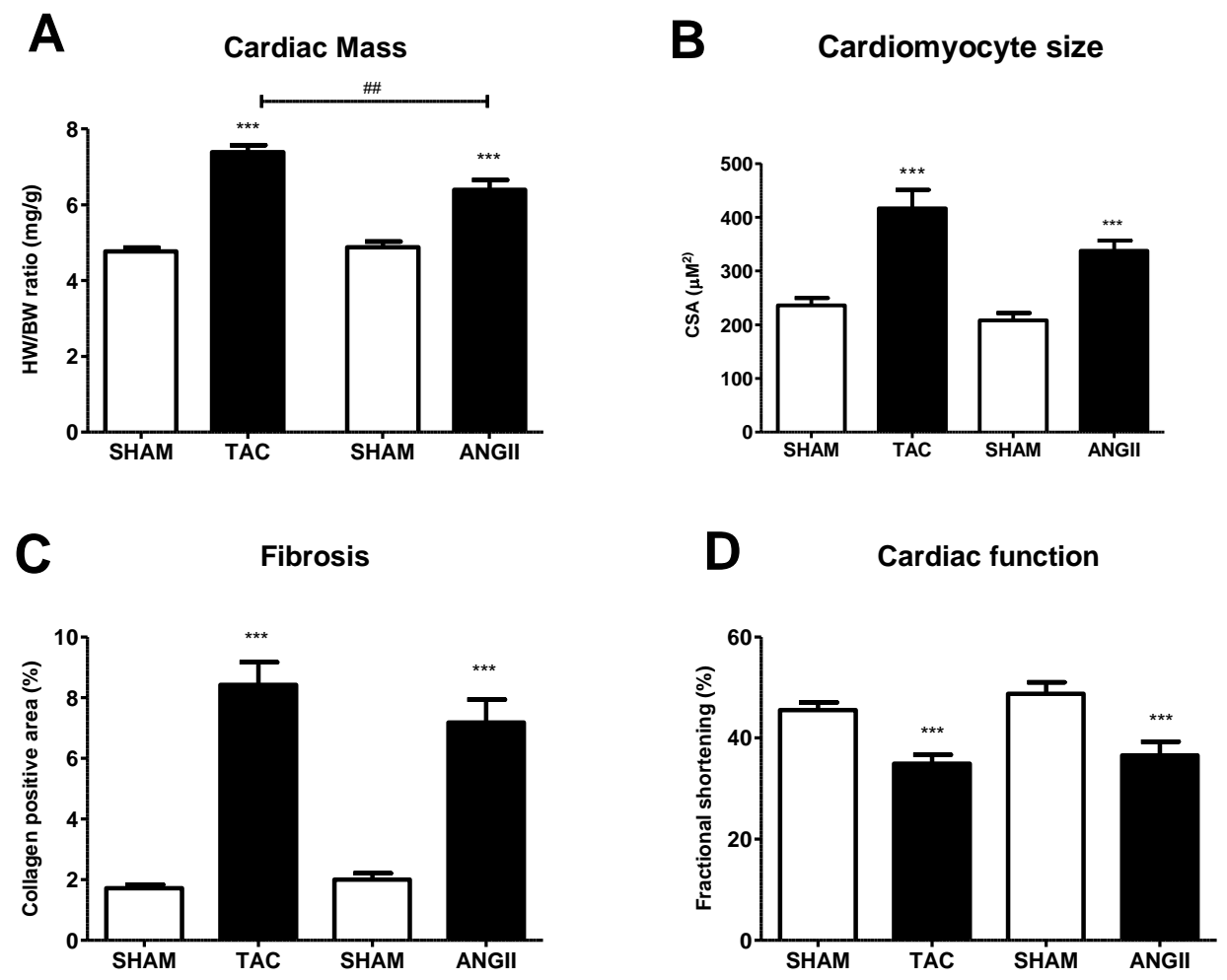

Figure 3. Comparison of cardiac phenotype induced by TAC and Angll in mice. The dataset was filtered to only include TAC $(n=69)$ and Angll studies $(n=23)$ having a duration of 4 weeks. Data are presented as means and SEM. Showing in panel A. HW/BW ratio. B. Cardiomyocyte cross sectional area (CSA). C. Collagen positive area. D. Fractional shortening. ${ }^{* * *} P<0.001$ compared to corresponding Sham, ${ }^{\#} P<0.01$ compared to Angll.

\section{Hypertrophy and fibrosis correlate after PO}

Genetically modified mouse models are extensively used to investigate the molecular mechanisms involved in the pathogenesis of PO induced heart failure ${ }^{11}$. Most studies in our dataset use knockout (KO) or transgenic mice (TG) to target a specific molecular pathway putatively involved either in cardiac hypertrophy, fibrosis or inflammation. Here, we investigated how these three parameters correlate after PO. We calculated fold changes for all OCPs induced by the experimental conditions for each study. Using these fold changes, we explored the relation between these parameters after experimental intervention. As expected, parameters associated with the same pathophysiological process (cardiac hypertrophy), cardiac mass and CSA correlate strongly $\left(R^{2}=0.64\right.$. $P<0.001$, Supplemental 
Figure 3). By presenting the effects of a genetic manipulation on the level of PO-induced hypertrophy and fibrosis (expressed as fold change) in a scatter plot (Figure 4A), it is immediately evident that the processes of hypertrophy and fibrosis closely correlate $\left(R^{2}\right.$ $=0.58, \mathrm{P}<0.0001)$.

\section{Inflammation in relation to cardiac hypertrophy and fibrosis}

Next, we combined the general conclusion on inflammation with the conclusion regarding hypertrophy and fibrosis, to determine the relation between these processes. Table 4 shows that changes in inflammation affect the other pathophysiological processes in the same direction, suggesting a close relation between inflammation, on the one hand, and cardiac hypertrophy and fibrosis, on the other.

Because numerical data on inflammation is limited in our dataset and the outcome measures used are diverse, in Figure $4 \mathrm{~B}$ we used colours to indicate the effect on cardiac inflammation. This clearly demonstrates that inflammation is following the change in both hypertrophy and fibrosis. In addition, in those studies in which leukocyte infiltration was quantified, the fold change in number of macrophages correlated with the fold change in cardiac mass and fibrosis after PO (Figure 4C-D).

Table 4. General outcome of all studies on cardiac hypertrophy, fibrosis and inflammation

\begin{tabular}{|c|c|c|c|c|}
\hline \multicolumn{5}{|c|}{ Hypertrophy vs. Fibrosis ${ }^{+}$} \\
\hline & $\begin{array}{c}\text { Fibrosis } \uparrow \\
(n=77)\end{array}$ & $\begin{array}{c}\text { Fibrosis - } \\
(n=49)\end{array}$ & $\begin{array}{c}\text { Fibrosis } \downarrow \\
(n=188)\end{array}$ & $N D(n=55)$ \\
\hline Hypertrophy $\uparrow(n=82)$ & 61 & 12 & $1^{*}$ & 8 \\
\hline Hypertrophy - $(n=85)$ & 13 & 24 & 35 & 12 \\
\hline Hypertrophy $\downarrow(n=177)$ & $2^{*}$ & 11 & 144 & 22 \\
\hline$N D(n=25)$ & 1 & 2 & 9 & 13 \\
\hline \multicolumn{5}{|c|}{ Hypertrophy vs. Inflammation ${ }^{\dagger}$} \\
\hline & $\begin{array}{c}\text { Inflammation } \uparrow \\
(\mathrm{n}=27)\end{array}$ & $\begin{array}{l}\text { Inflammation - } \\
(n=24)\end{array}$ & Inflammation $\downarrow(n=108)$ & ND $(n=210)$ \\
\hline Hypertrophy $\uparrow(n=82)$ & 19 & 9 & $3^{*}$ & 51 \\
\hline Hypertrophy - (n=85) & 7 & 8 & 27 & 43 \\
\hline Hypertrophy $\downarrow(n=177)$ & 0 & 4 & 70 & 102 \\
\hline$N D(n=25)$ & 1 & 3 & 7 & 14 \\
\hline \multicolumn{5}{|c|}{ 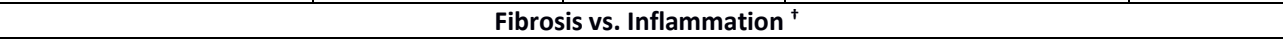 } \\
\hline & $\begin{array}{c}\text { Fibrosis } \uparrow \\
(n=77)\end{array}$ & $\begin{array}{c}\text { Fibrosis - } \\
(n=49)\end{array}$ & $\begin{array}{c}\text { Fibrosis } \downarrow \\
(n=188)\end{array}$ & ND $(n=55)$ \\
\hline Inflammation $\uparrow(n=27)$ & 19 & 4 & $1^{*}$ & 4 \\
\hline Inflammation - $(n=24)$ & 3 & 10 & 4 & 6 \\
\hline Inflammation $\downarrow(n=108)$ & $1^{*}$ & 11 & 87 & 8 \\
\hline$N D(n=210)$ & 56 & 22 & 94 & 37 \\
\hline
\end{tabular}

All studies (rat and mouse) with control and experimental groups after PO (groups 3 and 4 described in methods) are included. Number of studies are indicated per general conclusion: $\uparrow=$ increase, $-=$ No significant difference, $\downarrow=$ Reduced, ND = No data available. + statistically significant according to Fisher's test. Strikingly, a number of studies show opposite changes, e.g., a decrease in inflammation accompanied with an increase in hypertrophy ${ }^{21-23}$. In a similar way, in two studies the experimental condition affects inflammation and fibrosis in the opposite direction ${ }^{21,}$ ${ }^{24}$. All these studies are marked (*). Details of these studies can be found in Supplemental table 4. 

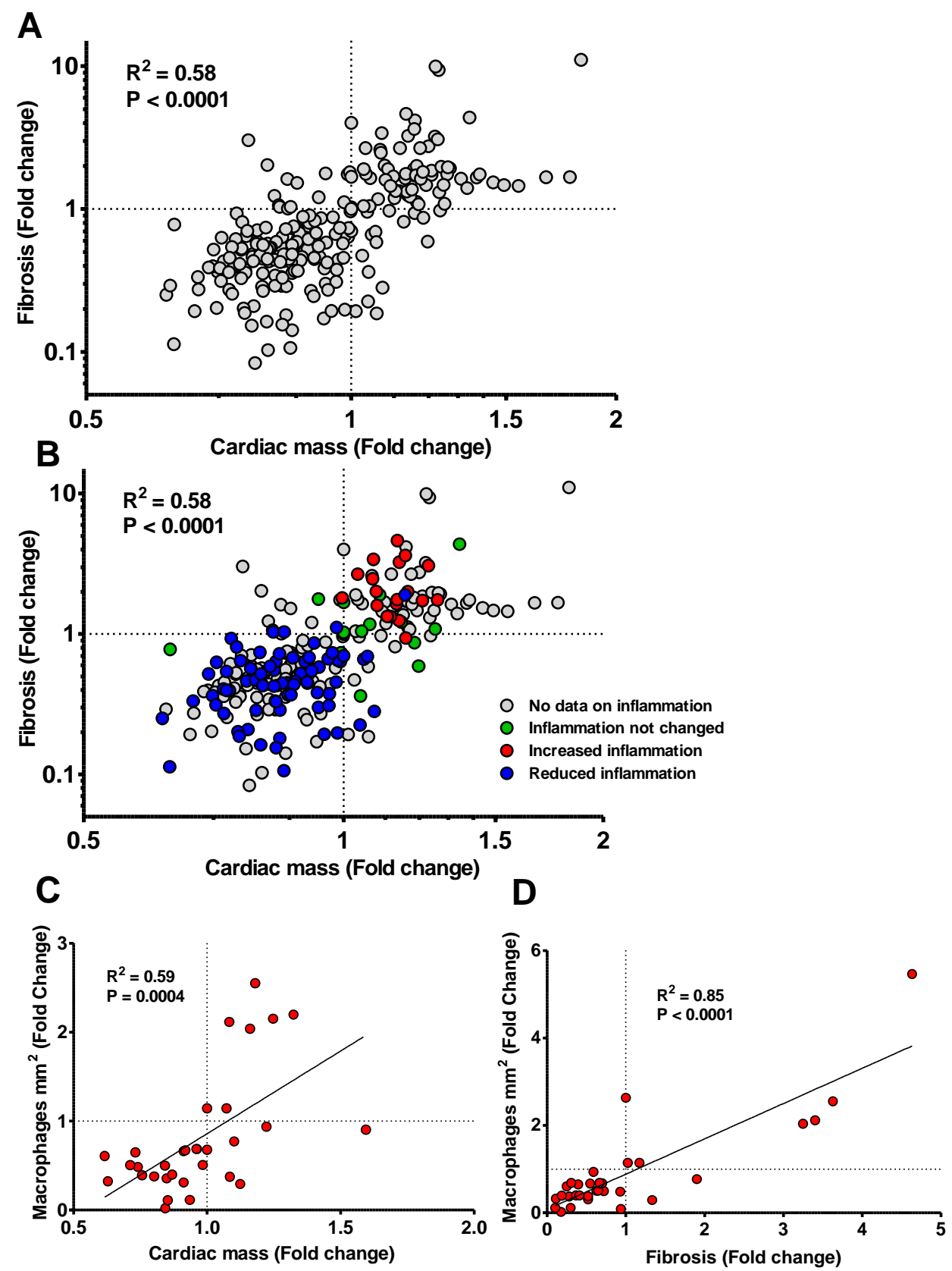

Figure 4. Inflammation correlates with the development of both cardiac hypertrophy and fibrosis. Panel $A$ shows the correlation between the fold change in cardiac mass and the fold change in fibrosis. In panel B the colour indicates the general conclusion on inflammation from this study in the same scatter plot. In panels C and D correlations between fold change in number of macrophages per $\mathrm{mm}^{2}$ and hypertrophy and fibrosis are shown. 


\section{Gene to Phenotype Interaction Network}

The gene to phenotype interaction network (GPIN) we created highlights the complex interactions between the three OCP processes. Our analysis included 253 studies that manipulated individual genes mostly through knockout or TG overexpression. Not all three OCPs were measured in all studies (Supplementary file 1). In fact, as shown in the Venn diagram (Figure $5 \mathrm{~A}$ ) the majority of studies derived from the literature database focused on two OCPs only, mostly fibrosis and hypertrophy. Due to overlaps between the studies this yielded 162 unique genes and mature miRNAs and 330 interactions after consolidating and confidence scoring for the creation of the GPIN. Figure 5B shows the GPIN, with the three OCPs in orange and genes coloured in blue. The pro interactions (172) are coloured in red and anti (158) in green. As Figure 5A indicates, the effect of manipulation of most genes is measured only on hypertrophy and fibrosis resulting in the majority of genes only affecting these OCPs. It is important to note that this is due to the lack in studies delivering this data instead of a representation of the actual biology at play.

\section{Identifying relevant miRNA regulators}

For further analysis of the interplay between the OCPs we merged multiple other interaction data sources (STRING, miRTarBase, TargetScan, miRDB and DIANA microT CDS) into the GPIN to add context (protein-protein interactions) and regulation (miRNA targeting). This increases the size of the network to 23024 nodes (protein coding genes, miRNAs, IncRNAs) and $2,978,343$ interactions. We used the GPIN to predict the relevance of individual miRNAs for the OCPs based on their gene targets and on the confidence score of the effect of these genes on the OCPs. This resulted in high scores for several miRNAs, among which, mmu-miR466l-3p, mmu-miR-124-3p, mmu-miR-30e-5p and mmu-miR-340-5p. Figure 6 shows miRNAs that score for at least one of the OCPs in the top $5 \%$ and their targets and OCPs. Table 5 lists the top 20 miRNAs ranked based on the sum of their individual OCP relevance scores. 
Table 5. Top 20 miRNAs relevant to pathophysiological processes in PO-induced HF. The score is based on the weight miRNA targeting interaction to genes in the network affecting the OCPs. As one miRNA targets multiple genes the scores are accumulated to calculate the relevance scores of a miRNA per OCP. The scores are scaled between 0 and 1 and summed for a general relevance score.

\begin{tabular}{|c|c|c|c|c|c|}
\hline & miRNA & Fibrosis Score & $\begin{array}{l}\text { Hypertrophy } \\
\text { Score }\end{array}$ & $\begin{array}{l}\text { Inflammation } \\
\text { Score }\end{array}$ & Accumulated Score \\
\hline 1 & mmu-miR-466l-3p & 1.00 & 1.00 & 1.00 & 3.00 \\
\hline 2 & mmu-miR-124-3p & 0.74 & 0.82 & 0.96 & 2.51 \\
\hline 3 & mmu-miR-30e-5p & 0.46 & 0.45 & 1.00 & 1.92 \\
\hline 4 & mmu-miR-340-5p & 0.58 & 0.63 & 0.69 & 1.90 \\
\hline 5 & mmu-let-7b-5p & 0.54 & 0.65 & 0.71 & 1.90 \\
\hline 6 & mmu-miR-19b-3p & 0.43 & 0.52 & 0.86 & 1.81 \\
\hline 7 & mmu-miR-384-5p & 0.43 & 0.43 & 0.91 & 1.78 \\
\hline 8 & mmu-miR-19a-3p & 0.41 & 0.50 & 0.82 & 1.73 \\
\hline 9 & mmu-miR-1961 & 0.50 & 0.56 & 0.66 & 1.72 \\
\hline 10 & mmu-miR-30d-5p & 0.41 & 0.42 & 0.87 & 1.70 \\
\hline 11 & mmu-miR-30c-5p & 0.41 & 0.42 & 0.87 & 1.70 \\
\hline 12 & mmu-miR-30b-5p & 0.41 & 0.42 & 0.87 & 1.70 \\
\hline 13 & mmu-miR-30a-5p & 0.41 & 0.42 & 0.87 & 1.70 \\
\hline 14 & mmu-let-7e-5p & 0.48 & 0.56 & 0.64 & 1.68 \\
\hline 15 & mmu-miR-325-3p & 0.60 & 0.57 & 0.48 & 1.65 \\
\hline 16 & mmu-let-7d-5p & 0.46 & 0.54 & 0.64 & 1.64 \\
\hline 17 & mmu-let-7i-5p & 0.47 & 0.54 & 0.62 & 1.62 \\
\hline 18 & mmu-miR-98-5p & 0.47 & 0.52 & 0.62 & 1.61 \\
\hline 19 & mmu-miR-27a-3p & 0.46 & 0.50 & 0.63 & 1.60 \\
\hline 20 & mmu-let-7g-5p & 0.45 & 0.52 & 0.62 & 1.59 \\
\hline
\end{tabular}




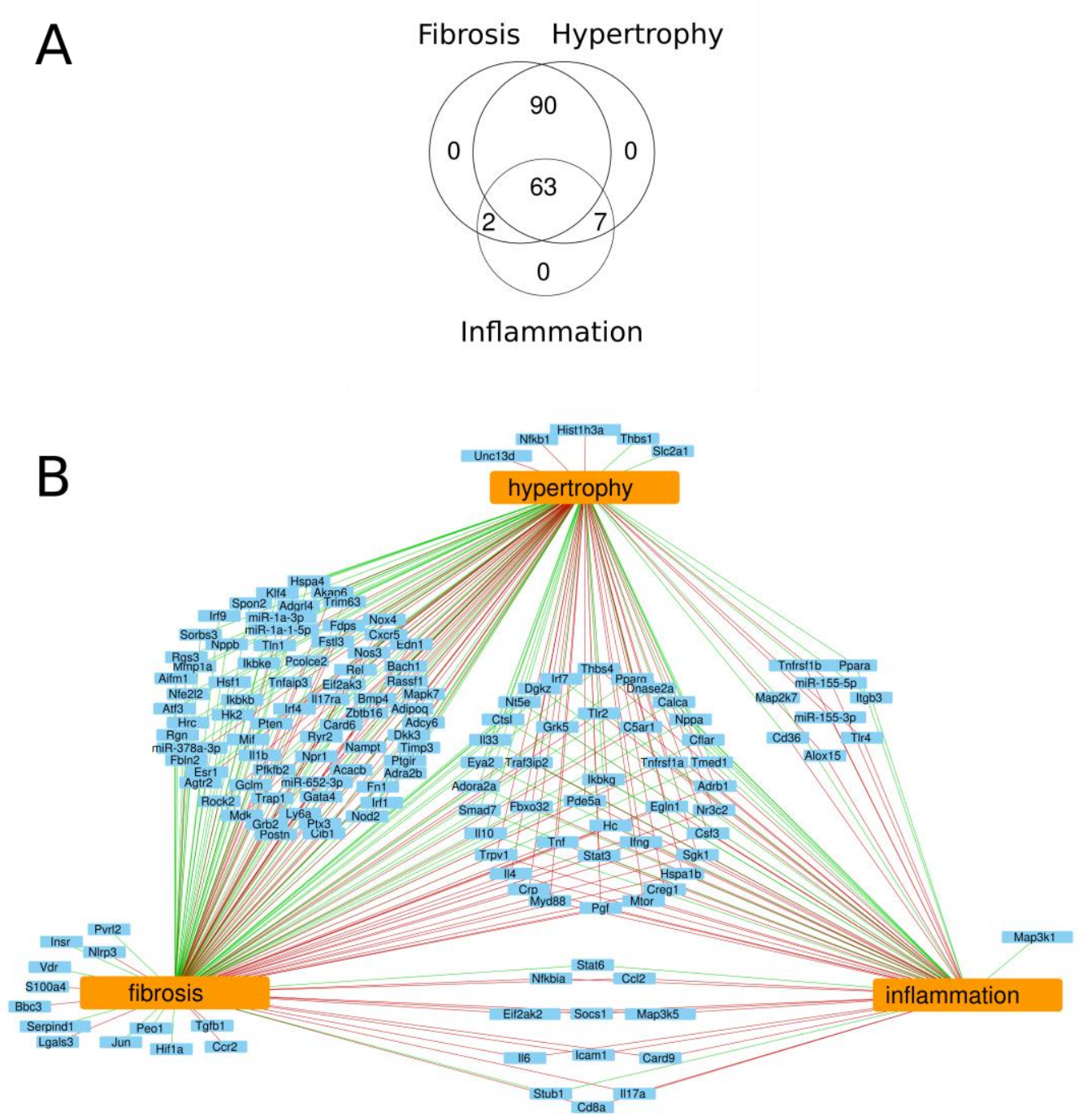

Figure 5. Gene to phenotype interaction network (GPIN). Panel A. Venn diagram showing how often each OCP was measured in all the studies used to build the GPIN. B. The genes that show an effect on at least one of the three OCPs. Red edges indicate a pro-effect and green edges an anti-effect. 


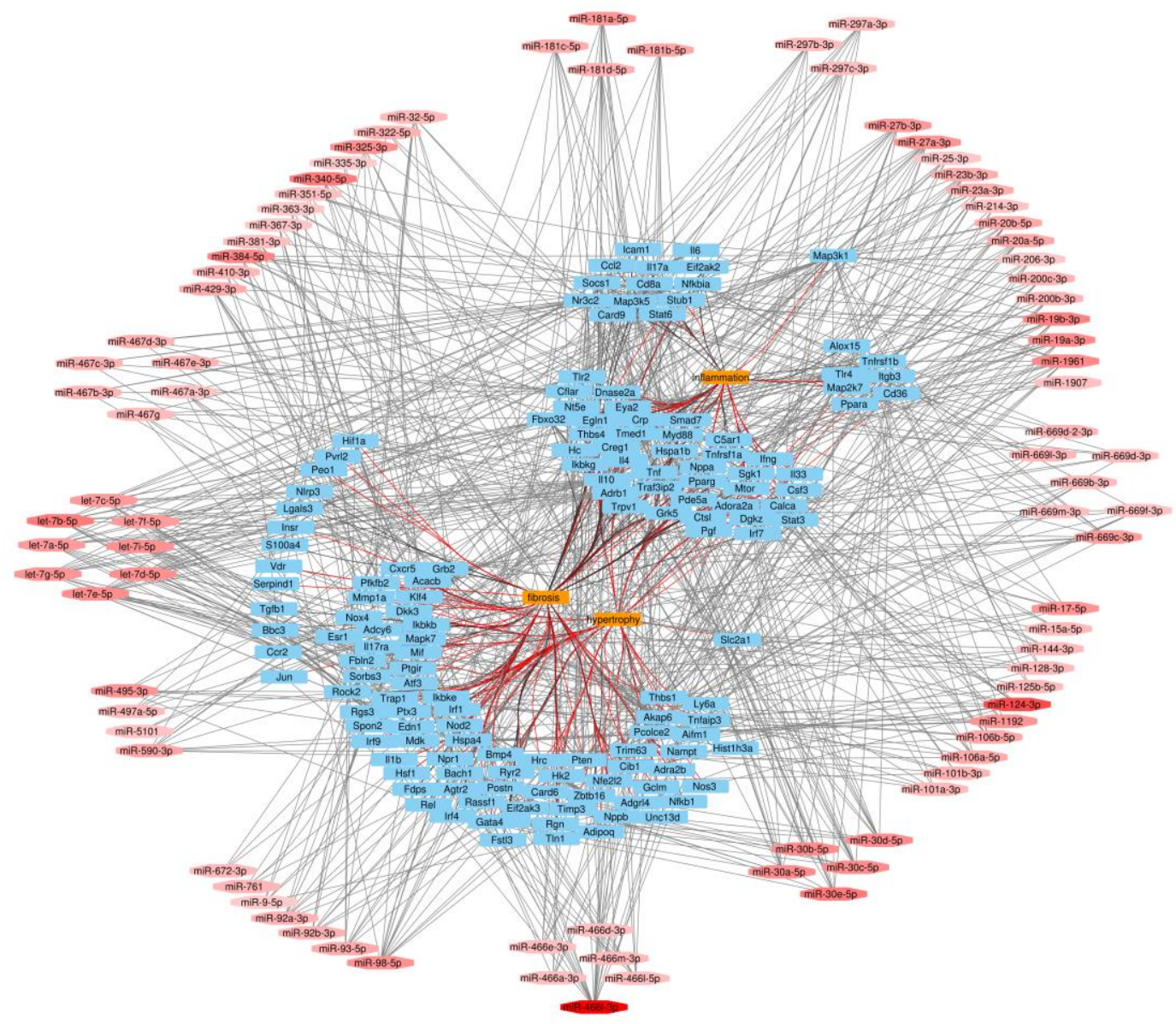

Figure 6. Extended GPIN network. Visualisation of the GPIN extended with miRNAs. OCPs (orange) are connected to genes (blue) that are targeted by the top 5\% most relevant miRNAs. The miRNAs are coloured according to their score. Red edges indicate a pro-effect and black edges an anti-effect, edges between miRNAs and genes are coloured grey. 


\section{Discussion}

The present study provides an unprecedented descriptive overview of pre-clinical cardiac PO studies published during the last 15 years. Animal models of PO-induced HF are vital for advancement of our understanding of the pathophysiology of hypertensive heart disease and aortic stenosis and play a crucial role in the development of new therapies ${ }^{11}$. By combining the results of 284 research articles we show that inflammation is closely linked with the pathophysiological processes of hypertrophy and fibrosis. In addition, we have constructed a gene-to-phenotype interaction network (GPIN) based on a systematic review of these preclinical studies. This approach of combining a systematic review with network analysis has to our knowledge never been used before. Using published data from articles the effect of individual genes on pathophysiological processes in $\mathrm{HF}$ was quantified. We have extended this network with miRNAs known (or predicted) to target the genes and identified miR-466I$3 p$, miR-124-3p, miR-30e-5p as potential therapeutic targets in PO induced HF. Finally, this dataset provides useful reference values to all researchers studying PO in animal models.

It is unclear to what extent the PO models of Angll infusion and TAC are comparable. One could argue that the Angll model better mimics hypertensive heart disease, while the TAC models shows more resemblance to aortic stenosis. The effects of Angll, however, are not limited to the development of cardiac PO due to increased hypertension only ${ }^{25}$ as Angll was shown to directly stimulate cardiomyocyte hypertrophy ${ }^{26}$. Moreover, Angll-induced systemic pro-inflammatory effects can play a role as well ${ }^{27}$. Analysis of the dataset points out that TAC induces a more profound increase in hypertrophy, as reflected by a larger increase in cardiac mass and cardiomyocyte size, whereas fibrosis and cardiac function do not differ between these PO models. These results indicate that the Angll model is milder in terms of hypertrophy, or alternatively, that the Angll model is characterized by more fibrosis, at a given level of hypertrophy.

We explored the relation between three pathophysiological parameters of POinduced HF, hypertrophy, fibrosis and inflammation. Our analysis showed that an experimental condition that significantly changes one of the OCPs often induces a parallel change in the other parameters. Noteworthy are the few studies where the pathophysiological parameters are changing in opposite directions. Cai et al. ${ }^{22}$, for example, showed that inhibition of HSP70 expression enhanced myocardial hypertrophy but suppressed cardiac fibrosis as induced by aortic constriction. Increased hypertrophy is initiated via the role of HSP70 in calcium handling, on the other hand, reduced fibrosis is mediated via a reduction in the inflammatory response as extracellular HSP70 attenuates the conjugation of HSP70 with TLR4, and thereby decreases macrophage infiltration and the inflammatory cytokines expression, which attenuates the pressure overload-induced cardiac fibrosis.

HF has been shown to be associated with inflammation ${ }^{28}$ and inflammation is believed to play an active role in HF disease progression ${ }^{29}$. Infiltrating inflammatory cells affect both cardiac hypertrophy and fibrosis ${ }^{5}$. It is interesting to speculate whether the process of inflammation is a cause or a consequence of HF. Regretfully, the number of studies where inflammation is numerically quantified is limited. In contrast to hypertrophy and fibrosis, inflammation is neither stringently defined nor measured in a consistent manner. Next to the infiltration of immune cells, the presence and degree of inflammation is monitored by the secretion of cytokines or the activation of intracellular inflammatory pathways, such as the NFkB pathway. In addition, the diverse set of measurements used to 
quantify the inflammatory processes limits the comparability of studies. We found the number of macrophages per $\mathrm{mm}^{2}$ of myocardium to be the most frequently used parameter to determine cardiac inflammation. In our dataset only 46 studies (12\%) included this measurement. Together, these data support the notion that inflammation correlates with adverse disease progression in $\mathrm{HF}$.

Numerous studies in the dataset target specific components of the inflammatory response, e.g. key pro-inflammatory cytokines such as, TNF ${ }^{30,31}{ }^{31}$ IL-6 ${ }^{32-34}$ or IFNY ${ }^{35,36}$. Deletion of these cytokines in these specific studies leads to a reduction in fibrosis and hypertrophy, thereby supporting the notion that inflammation plays an active role in the development of cardiac fibrosis and hypertrophy after PO. Nevertheless, it remains difficult to distinguish if inflammation is a cause or merely an effect of HF. To our surprise in several studies that manipulated the immune response, inflammation itself was not quantified. For instance, Honsho et al. ${ }^{37}$ measured IL-1 $\beta$ secretion from stretched CF's in vitro, but did not measure cardiac inflammation in IL-1 $\beta$ KO mice subjected to TAC. In other papers, the role of the immune-related interferon regulatory factor (IRF) family members, IRF1 ${ }^{38}$, IRF4 ${ }^{39}$ and IRF9 ${ }^{40}$ in HF was explored, without actually quantifying the amount of inflammation in the heart.

\section{Network analysis and miRNAs}

Based on the number of genes in the GPIN that miRNAs are predicted to target, we calculated scores for all miRNAs. This novel approach allowed the identification of candidate miRNAs influencing cardiac inflammation, hypertrophy and fibrosis. The microRNA with the highest score for enrichment is mmu-miR-466l-3p. Indeed, it was shown that acute inflammation upregulates miR-466l, which in turn regulates resolution ${ }^{41}$, a process in which inflammation is cleared. Overexpression of miR-466l promotes specialized pro-resolving mediator production ${ }^{41}$ making mmu-miR-466I-3p, identified in our GPIN, an interesting candidate for anti-inflammatory treatment. miR-124-3p, the second highest scorer in in our analysis, was described to control the proliferative, migratory, and inflammatory phenotype of pulmonary vascular fibroblasts ${ }^{42}$ and to play a role in the pathogenesis of pulmonary arterial hypertension. Moreover, it was shown to suppress the NFAT signalling pathway ${ }^{43}$, known to be involved in cardiac hypertrophy. Interesting to mention as well are miR-19 and miR-340. miRNA-19a and 19b are members of the miR-17-92 cluster, which regulates the expression of the ECM proteins CTGF and TSP-1 in ageing-related heart failure as shown by our group previously ${ }^{44}$. A recent study by Zhu et al. ${ }^{45}$ used network analysis to identify HF related miRs ${ }^{45}$, based on expression levels of miRNAs and genes in myocardial biopsies of dilated cardiac myopathy (DCM) patients. Their expression data driven miRNA-Gene-Network identified miR-340 as an important player in HF. This is in line with our GPIN analysis, in which miR-340 was identified as one of the most relevant miRNAs. MiRNAs with highest scores for individual OCPs are putatively important in the development of these pathophysiological processes. However, for many of these miRNAs it remains to be established if they are expressed in the heart and, if so, if their expression changes during $\mathrm{HF}$ development. Moreover, our scoring approach favours miRNAs that have many targets. MiR466I-3p in particular has 3196 predicted target genes in the miRNA targeting databases we integrated in the GPIN. Although potentially interesting as therapeutic target, this considerably increases the chance of unwanted side effects. 


\section{Systematic reviews of animal studies and publication bias}

Previously others have performed meta-analysis of animal studies, also in the cardiovascular field ${ }^{8,46}$. These meta-analyses are based on a much smaller number of studies, and focus on a specific treatment and effect size. A relevant question is to what extent publication bias effects the conclusions of the present study. In animals studies, the number of publications presenting negative results is generally low, as was demonstrated by a systematic review in animal studies of acute ischaemic stroke ${ }^{47}$, where only $2 \%$ of the included publications reported no significant effects ${ }^{47}$. In contrast to many other systematic reviews we did not focus on the effect size of a specific treatment or intervention. Generally speaking, that type of analysis is more prone to publication bias ${ }^{48}$. As our PO comparisons and reference values are mostly based on wild-type mice potential effects of publication bias are most likely limited. Moreover, as the articles in our dataset measure more than one outcome parameter the incorporation of neutral data is more common. This is reflected by the relatively high number of studies that report no significant difference in one of the three OCPs we focussed. Nonetheless, the scatterplot in Figure 4A clearly shows two clouds of data points and a void around the area reflecting no change, suggesting that neutral effect sizes are underrepresented ${ }^{48}$. Accordingly, we cannot entirely rule out that there is some effect of publication bias on outcome.

\section{Conclusion}

In conclusion, we established a novel approach to identify potential HF therapeutic targets by using existing literature to construct a Gene-to-Phenotype interaction network. In this way we identified several potentially interesting miRNAs. Secondly, we explored the relation between inflammation, on the one hand, and hypertrophy and fibrosis, on the other, and showed that inflammation strongly correlates with the pathophysiological processes of hypertrophy and fibrosis, supporting the concept that inflammation plays a central role in $\mathrm{HF}$ development.

\section{References}

(references combined and included with supplemental material) 


\section{Supplementary Methods}

\section{Detailed search terms and strategy:}

Disease model for pressure overload:

pressure overload [TIAB] or TAC[TIAB] or (aortic constriction[TIAB]) or Angiotensin I[TIAB] or Angiotensin II[MeSH] or (ANG II[TIAB]) or ((spontaneous and hypertensive) or (spontaneously and hypertensive))

Species:

Mouse [mesh] OR Rat [MeSH]

Selection of at least 2 of the three outcome parameters:

Fibrosis and inflammation: (((fibrosis[MeSH]) OR fibrosis[tiab]) and (inflammation[MeSH] or inflammation[tiab] or (inflammatory response[tiab])))

Fibrosis and hypertrophy: (((fibrosis[MeSH]) OR fibrosis[tiab]) and ((Cardiomyopathy, Dilated[mesh]) or (Hypertrophy, Left Ventricular[mesh]) or cardiac hypertrophy[MeSH]])

Inflammation and hyperthrophy: ((inflammation[MeSH] or inflammation[tiab] or (inflammatory response[tiab])) and ((Cardiomyopathy, Dilated[mesh]) or (Hypertrophy, Left Ventricular[mesh]) or cardiac hypertrophy[MeSH])))

Organ:

heart [MeSH]

Filters for publication date and language:

Date (01-01-2000 until 31-12-1014) and (English).

The combined search term used in PubMed:

((()Mouse [mesh] OR Rat [MeSH]) AND ( (((fibrosis[MeSH]) OR fibrosis[tiab]) and (inflammation[MeSH] or inflammation[tiab] or (inflammatory response[tiab]))) OR (((fibrosis[MeSH]) OR fibrosis[tiab]) and ((Cardiomyopathy, Dilated[mesh]) or (Hypertrophy, Left Ventricular[mesh]) or cardiac hypertrophy[MeSH])) OR ((inflammation[MeSH] or inflammation[tiab] or (inflammatory response[tiab])) and ((Cardiomyopathy, Dilated[mesh]) or (Hypertrophy, Left Ventricular[mesh]) or cardiac hypertrophy[MeSH]))) ) AND (pressure overload [tiab] or (aortic constriction) or TAC[tiab] or Angiotensin II[tiab] or Angiotensin II[mesh] or (ANG IItiab]) or (spontaneous and hypertensive) or (spontaneously and hypertensive)) AND heart [MeSH])) AND ("2000/01/01"[Date - Publication] : "2014/12/31"[Date Publication])

\section{Data analysis and processing}

Our approach was based on the methods of a meta-analysis, using a similar search protocol and strict inclusion criteria. In the data extraction, processing and visualisation we deviated from the approach used in a meta-analysis, by visualizing outcome parameters extracted from individual studies as data points in our plots instead of using forest plots. Standard deviations or other statistical measures of variance were not extracted from the studies, because it was not feasible to obtain these values from the graphics in many papers in a reliable way. Moreover, we felt that the sheer number of studies allows statistical analysis based on the mean values extracted.

\section{Normalization of numerical parameters}

In the various studies similar OCPs were assessed, normalized and presented in different ways. Cardiac mass for example was presented normalized for either body weight (BW) or for tibia length (TL). Cardiomyocyte size was usually presented as cross sectional area (CSA, $\mu \mathrm{m}^{2}$ ), but sometimes also as diameter $(\mu \mathrm{m})$. OCPs of cardiac mass, cardiomyocyte size and fibrosis were normalized to allow direct comparison of these outcome measures between the different studies in our analyses.

Cardiac Mass: In the literature cardiac mass was presented as: $\mathrm{HW} / \mathrm{BW}$ ratio $(\mathrm{mg} / \mathrm{g})$, $\mathrm{LVw} / \mathrm{BW}$ ratio $(\mathrm{mg} / \mathrm{g}), \mathrm{HW} / \mathrm{TL}$ ratio $(\mathrm{mg} / \mathrm{mm}), \mathrm{LVw} / \mathrm{TL}$ ratio $(\mathrm{mg} / \mathrm{mm})$, Absolute $\mathrm{HW}(\mathrm{mg})$, Absolute $\mathrm{LVw}(\mathrm{mg})$ and as relative increase vs. SHAM. All these values were converted to $\mathrm{HW} / \mathrm{BW}$ ratio, being the most frequently used measure. Corrections for TL towards BW were 
calculated using a correction factor based on historical data from the Jackson laboratory phenome database ${ }^{49}$ (http://phenome.jax.org/).

Cardiomyocyte size: Cardiomyocyte size was most often presented as CSA $\left(\mu \mathrm{m}^{2}\right)$, but also as myocyte diameter $(\mu \mathrm{m})$ or as Arbitrary Units (AU). Myocyte diameter was converted to CSA based on the area of a circle and scaled based on the mean SHAM level to mean CSA SHAM level ratio. We have corrected Myocyte diameter $\mu \mathrm{m}$ into CSA by using the following formula: (Diameter $/ 2)^{2} \times \pi \times 1.7$. Based on the area of a circle but multiplied by a factor 1.7 to scale the mean of SHAM groups levels to the mean CSA SHAM levels ( $244 \mu \mathrm{m}^{2}$ ).

Fibrosis area: Reported baseline values for the percentage fibrosis in the myocardium ranged from $0 \%$ to $12.3 \%$. Studies reporting a very low percentage fibrosis at baseline, show a very large relative increase after PO. To avoid artificially high values for the relative increase when baseline fibrosis areas approached zero, baseline values below $1 \%$ were set to $1 \%$. An exception was made when values after PO were lower than $2 \%$ to avoid creating an artificial decrease after PO.

Macrophages per $\mathbf{m m}^{2}$ : The only inflammation related parameter of which numerical data was extracted is the number of macrophages per $\mathrm{mm}^{2}$ determined by immuno-histochemical staining. Although different markers for macrophages were used (For mice: CD68, Mac-2, Mac-3, F4/80 and for Rat: ED-1) we did not distinguish between them in the analysis.

Supplemental Table 1. Overview of study details and outcome parameters (OCPs) extracted.

\begin{tabular}{|c|c|c|}
\hline General information & Experimental details & Extracted outcome data \\
\hline \multirow{2}{*}{$\begin{array}{l}\text { Name first } \\
\text { author }\end{array}$} & \multirow{14}{*}{$\begin{array}{l}\text { Species of Animals } \\
\text { Gender } \\
\text { Strain } \\
\text { Duration } \\
\text { Age at start } \\
\text { Number of animals used } \\
\text { Use of KO/TG animals } \\
\text { Gene ID of manipulated } \\
\text { gene } \\
\text { PO model } \\
\text { Infused dose (Angll / ISO) } \\
\text { Size of constriction (AC) }\end{array}$} & Hypertrophy \\
\hline & & Measure of cardiac mass (HW/BW) \\
\hline Year of & & Measure of cardiomyocyte size (CSA) \\
\hline publication & & Measure of wall thickness (LVPWd) \\
\hline & & General conclusion ( $\downarrow-)$ \\
\hline Title & & \\
\hline & & Cardiac Function \\
\hline PubMed ID & & Measure of cardiac function (EF/FS\%) \\
\hline Journal & & Measure of Blood pressure (SBP) \\
\hline Jourlidi & & General conclusion ( $\downarrow$-) \\
\hline \multirow[t]{4}{*}{ Brief description } & & Fibrosis \\
\hline & & $\begin{array}{l}\text { Measure of collagen/fibrotic area (\%) } \\
\text { General conclusion ( } \downarrow \downarrow-)\end{array}$ \\
\hline & & Inflammation \\
\hline & & $\begin{array}{l}\text { Macrophages number (cells per } \mathrm{mm}^{2} \text { ) } \\
\text { General conclusion ( } \uparrow \downarrow-\text { ) }\end{array}$ \\
\hline
\end{tabular}

Outcome parameters are mostly numerical data, except for the general conclusions on the articles on the processes hypertrophy, function, fibrosis and inflammation. 


\section{Supplemental Results}

\section{Introduction}

Understanding of the pathogenesis of HF requires a detailed characterisation of the underlying pathophysiological processes and of the different disease aetiologies, such as hypertensive heart disease and aortic stenosis. Rodent models are extensively used to investigate the molecular mechanisms involved in the pathogenesis of PO-induced heart failure ${ }^{11}$. In this supplemental section, our dataset, composed of 369 studies, in which over 16 thousand animals were used in total, was used to compare features of frequently used rodent PO models (Supplemental Table 2). Despite extensive use of these preclinical HHD models, the similarities and dissimilarities are ill-defined and reference values for the expected degree of hypertrophy, fibrosis and inflammation have not been reported so far. Moreover, the combined studies in our dataset allow a detailed analysis of the temporal changes induced by PO in the mouse heart. Collectively, this provides deeper insight into the interpretation of performed studies and can improve the design of future PO studies, thereby potentially reducing animal numbers needed in the future.

Supplemental table 2. Overview of most common mouse and rat models of pressure overload.

\begin{tabular}{|c|c|c|c|c|c|}
\hline \multicolumn{2}{|c|}{ Pressure overload model } & \multirow{2}{*}{$\begin{array}{l}\text { Brief description } \\
\begin{array}{l}\text { Banding of the } \\
\text { abdominal aorta }\end{array}\end{array}$} & \multirow{2}{*}{$\begin{array}{l}\text { Variables } \\
\text { Size of } \\
\text { constriction }\end{array}$} & \multirow{2}{*}{$\begin{array}{l}\text { Species } \\
\text { Rat }\end{array}$} & \multirow{2}{*}{$\begin{array}{l}\text { (dis)advantages } \\
\text { Induces potent hypertrophic } \\
\text { response. Invasive surgery. }\end{array}$} \\
\hline \multirow{2}{*}{$\begin{array}{l}\text { Aortic } \\
\text { constriction }\end{array}$} & $A A C$ & & & & \\
\hline & TAC & $\begin{array}{l}\text { Banding of the } \\
\text { transverse aorta }\end{array}$ & $\begin{array}{l}\text { Size of } \\
\text { constriction }\end{array}$ & Mouse & $\begin{array}{l}\text { Induces potent hypertrophic } \\
\text { response. Invasive surgery. }\end{array}$ \\
\hline \multicolumn{2}{|c|}{ Angiotensin II infusion } & $\begin{array}{l}\text { Infusion via osmotic } \\
\text { mini-pumps }\end{array}$ & $\begin{array}{l}\text { Dose and } \\
\text { Duration }\end{array}$ & $\begin{array}{l}\text { Mouse, } \\
\text { Rat }\end{array}$ & $\begin{array}{l}\text { Systemic elevation of BP, No } \\
\text { invasive surgery }\end{array}$ \\
\hline \multicolumn{2}{|c|}{ Isoproterenol infusion } & $\begin{array}{l}\text { Infusion via osmotic } \\
\text { mini-pumps }\end{array}$ & $\begin{array}{l}\text { Dose and } \\
\text { duration }\end{array}$ & $\begin{array}{l}\text { Mouse, } \\
\text { Rat }\end{array}$ & $\begin{array}{l}\text { No invasive surgery, hypertrophy } \\
\text { along with myocardial damage. }\end{array}$ \\
\hline \multicolumn{2}{|c|}{$\begin{array}{l}\text { Salt-diet in salt sensitive } \\
\text { rats }\end{array}$} & $\begin{array}{l}\text { Salt added to diet / } \\
\text { drinking water }\end{array}$ & $\begin{array}{l}\text { Duration, } \\
\text { genetic } \\
\text { background }\end{array}$ & Rat & $\begin{array}{l}\text { Clinically relevant model, Gradual } \\
\text { onset of hypertension. }\end{array}$ \\
\hline \multicolumn{2}{|c|}{ Genetic background } & $\begin{array}{l}\text { Genetic trait } \\
\text { associated with } \\
\text { hypertension. }\end{array}$ & $\begin{array}{l}\text { Genetic } \\
\text { background, } \\
\text { transgene, } \\
\text { age }\end{array}$ & Rat & $\begin{array}{l}\text { No invasive surgery, or } \\
\text { pharmacological intervention. } \\
\text { Gradual onset of hypertension. } \\
\text { Control animals often a problem. }\end{array}$ \\
\hline
\end{tabular}




\section{Results and Discussion}

\section{Cardiac features of rats and mice}

Analysis revealed baseline differences in several outcome parameters (OCPs) between rat and mice. In Supplemental Table 3 we defined reference values for (patho)physiological parameters, such as hypertrophy, fibrosis, cardiac function, blood pressure and inflammation in both rat and mouse. Strikingly, average baseline cardiomyocyte surface area (CSA) values reported for mice are lower for mice than for rat. Reported baseline values for fractional shortening ( $48 \pm 12 \%$ ) can be used as reference values. Baseline systolic blood pressure (SBP) is somewhat higher in rats than mice. Differences in collagen area at baseline and after PO between species were not observed. The number of infiltrated macrophages shows an enormous variation between studies, and is therefore difficult to compare. Because of the limited number of rat studies and the variation in the PO models applied (Table 4), we focused on mice studies only for further analysis.

Supplemental Table 3. Overview of reference values of outcome parameters in baseline and PO conditions for wild-type rat and mouse of all strains.

\begin{tabular}{lcccc}
\hline & \multicolumn{2}{c}{ Rat } & \multicolumn{2}{c}{ Mouse } \\
Parameter & Sham & PO & Sham & PO \\
\hline HW/BW Ratio $(\mathrm{mg} / \mathrm{g})$ & $2.9 \pm 0.49$ & $4.13 \pm 0.93$ & $4.6 \pm 0.7$ & $6.9 \pm 1.4$ \\
CSA $\left(\mu \mathrm{m}^{2}\right.$ ) & $308.2 \pm 114.3$ & $538.7 \pm 242.8$ & $244 \pm 15$ & $400 \pm 25$ \\
Fractional Shortening (\%) & $48.2 \pm 12.5$ & $\mathrm{~N} / \mathrm{A}$ & $48.6 \pm 12.6$ & $\mathrm{~N} / \mathrm{A}$ \\
LVPW(d) (mm) & $1.5 \pm 0.4$ & $2.0 \pm 0.5$ & $0.9 \pm 1.3$ & $1.2 \pm 1.4$ \\
Fibrosis (\% area) & $2.2 \pm 2.1$ & $8.4 \pm 3.5$ & $1.9 \pm 1.7$ & $7.9 \pm 2.9$ \\
Macrophages per $\mathrm{mm}^{2}$ & $48.8 \pm 103.0$ & $421.1 \pm 2005.4$ & $42.1 \pm 111.4$ & $232.6 \pm 669.2$ \\
SBP (mm Hg) & $118.6 \pm 15.5$ & $\mathrm{~N} / \mathrm{A}$ & $105.9 \pm 12.4$ & $\mathrm{~N} / \mathrm{A}$ \\
\hline
\end{tabular}

Values are represented as the mean with standard deviation. N/A: Values that are irrelevant without subgroup analysis due to large variation in study design.

\section{Cardiac hypertrophy, but not fibrosis, correlates with PO duration}

Supplemental Figure 1 shows the fold change for OCPs between sham and PO conditions plotted against the duration of the experiment. Cardiac mass correlates positively with the duration of the experiment, $\left(R^{2}=0.33, P<0.0001\right)$, characterized by a rapid increase in cardiac mass in the first weeks after PO which seems to stabilize after 21 days (Supplemental Figure 1A). Cardiomyocyte size shows a more or less similar pattern, albeit with far more variation between data points (Supplemental Figure 1B). Interestingly, no correlation was observed between the degree of fibrosis and the duration of the experiment (Supplemental Figure 1C). Cardiac function was found to decline $\left(R^{2}=0.19, P<0.0001\right)$ as a function of the duration of the experiment (Supplemental Figure 1D). It is noteworthy, however, that some studies (13\%) actually report an increase in fractional shortening upon PO, suggesting a compensatory response in systolic cardiac function under some conditions. 
A

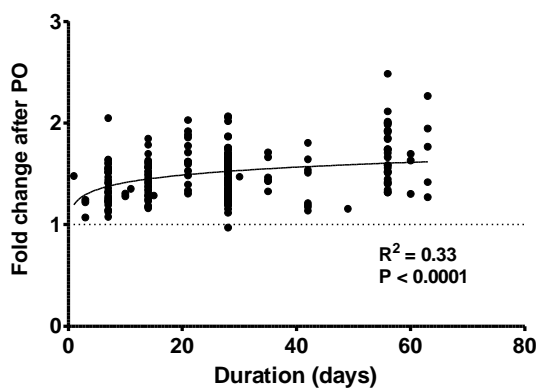

C

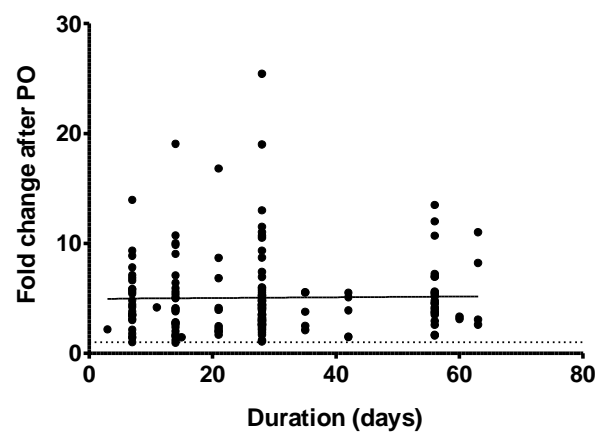

B

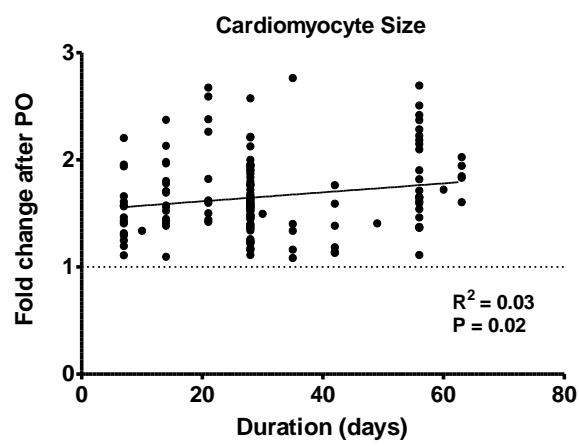

D

Fractional Shortening

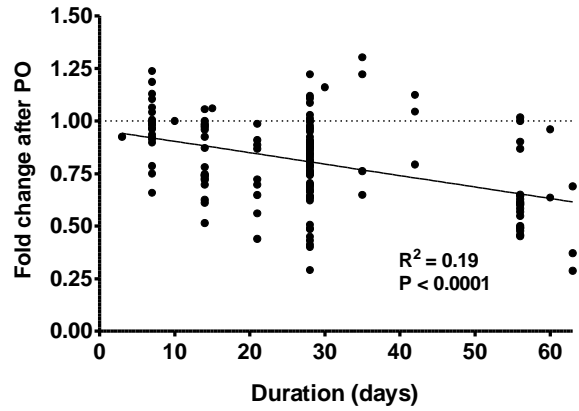

Supplemental figure 1. Temporal changes in cardiac phenotype in mice after PO. Mouse studies with TAC and Angll up to a duration of 63 days are included. A. Scatterplot showing fold increase in cardiac mass between sham and PO plotted against the duration of the experiment. B. Scatterplot showing fold change in cardiomyocyte size between sham and PO plotted against the duration of the experiment. C. Shows fold change in fibrosis for durations (binned). $D$. Scatterplot showing fold change in Fractional shortening between sham and PO plotted against the duration of the experiment. The dotted line indicates no difference in fold change.

Previously, a number of experimental studies provided data on the time course of changes in hypertrophy, fibrosis and inflammation after induction of $\mathrm{PO}{ }^{50-52}$. This allows a comparison of serial measurements performed by others, with the data generated in this systematic review. Histological examination of cardiac tissue at 3,7 and 28 days after TAC by Xia et al. ${ }^{50}$ indicates that cardiac mass as well as other parameters of hypertrophy increase with duration of the experiment. The infiltration of macrophages as well as cardiac fibrosis already peak at 7 days ${ }^{50}$. Interestingly, in our analysis the amount of fibrosis shows no linear increase either (Supplemental figure 1C). It is interesting to speculate whether the balance between collagen synthesis and degradation shifts as a function of time, as collagen degradation is induced by the infiltrating pro-inflammatory cells ${ }^{53}$. Well in line with our analysis (Supplemental figure 1D) cardiac function (fractional shortening) showed a steady linear decrease over time, becoming significant only after 28 days in the study of Xia and colleagues ${ }^{50}$. Rizzi and colleagues have studied temporal changes in cardiac matrix metalloproteinase activity after 15,30 and 75 days of $\mathrm{PO}{ }^{51}$. They reported a progressive increase in cardiac hypertrophy in time and measured highest collagen area at 15 days, indicating once more that collagen is degraded in later stages of disease. Stewart et al., on 
the other hand investigated fibroblast function after PO and showed collagen content to increase continuously 7,14 and 28 days after abdominal aortic constriction ${ }^{52}$. Others have studied the variety in the phenotype induced by TAC ${ }^{54}$ demonstrating that mice have lower ejection fraction at 3 or 9 weeks post-TAC, but that only a $28 \%$ of these mice develop HF.

The strength of our approach to characterize the PO-induced phenotype based on published articles is that the combination of results from hundreds of different labs corrects for the large variation in between labs due to animal handling, housing and other factors. The large number of studies combined gives a more accurate description of the PO induced phenotype. This allows better prediction of model variation and effect sizes, thereby reducing the number of animals needed for future studies.
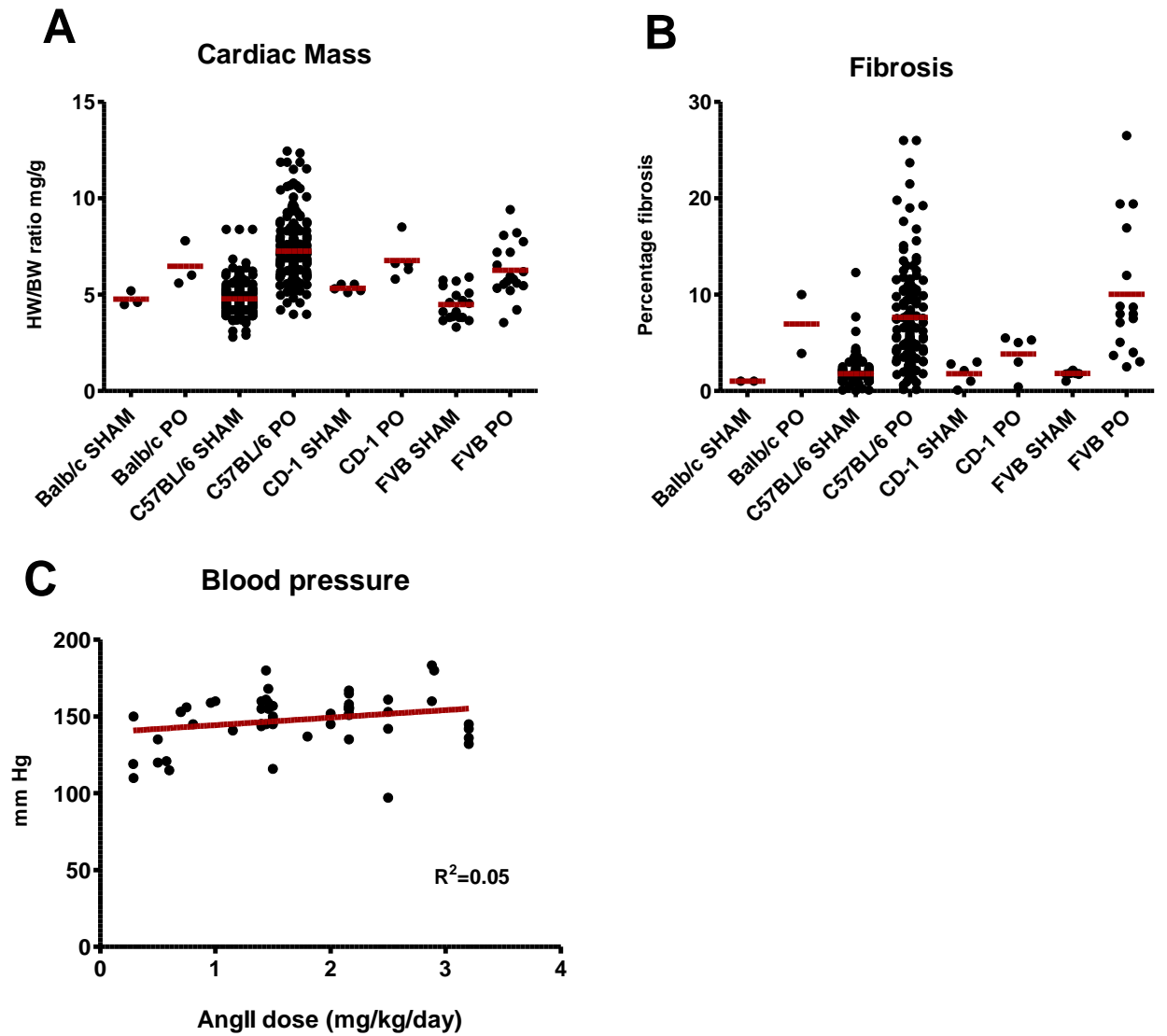

Supplemental figure 2. PO-induced cardiac phenotype in different mouse strains and used doses of Angll. $A$. $H W / B W$ ratios of TAC and Angll studies in different mice strain before and after PO. B. Percentage fibrosis in TAC and Angll studies in different mice strain before and after PO. C. Effect of Angll dose on blood pressure (SBP) showing only a modest and non-significant rise with increasing Angll doses. 


\section{Strains, dosing, blood pressure and other variables}

Clear phenotypical differences between the various mouse strains used in PO studies are not observed. Cardiac mass and fibrosis increased to a similar extent in C57BL/6, Balb/c, FVB and CD-1 mice (Supplemental Figure 2A-B). Supplemental Figure 2C shows the relation between the dose of Angll infused and the measured systolic blood pressure (SBP). Surprisingly, an Angll dose of $1 \mathrm{mg} / \mathrm{kg} /$ day suffices to achieve a pronounced increase in blood pressure (SBP) and the effect of higher doses on blood pressure seems limited.

There are many differences in experimental setup between studies. Studies vary in the use of strains, anaesthetics, size of constriction and Angll dose, etc., making comparisons difficult. This heterogeneity potentially explains differences in outcome. For example, it has been demonstrated that Angll infusion leads to dilated cardiomyopathy (DCM) in Balb/c mice, but not in C57BL/6 mice ${ }^{55}$. Notably, C57BL/6 mice of a different substrain (-J or $-\mathrm{N}$ substrain) have been shown to respond different to TAC ${ }^{56}$. Survival and cardiac function were significantly lower in the $-\mathrm{N}$ mice compared with $-\mathrm{J}$ mice ${ }^{56}$, stressing the importance of defining which substrain is actually used (Table 3). Even the type of mini-pump used for Angll infusion can lead to differences in blood pressure ${ }^{57}$. In order to enable better comparison of different studies, it would be useful to introduce further standardization of animal experimental conditions.

\section{Conclusion}

Systematic review of the literature shows that cardiac mass and cardiomyocyte size increase predominantly in the first weeks after PO, whereas cardiac function continues to decrease steadily over the next weeks. Surprisingly, the level of fibrosis hardly increases with time. The obtained reference values help to predict the model variation and effect sizes which, in turn, can help to reduce the number of animals needed in future studies. The current analysis also highlights the importance of detailed and proper documentation of all experimental conditions in order to increase reproducibility and interpretation of animal studies.

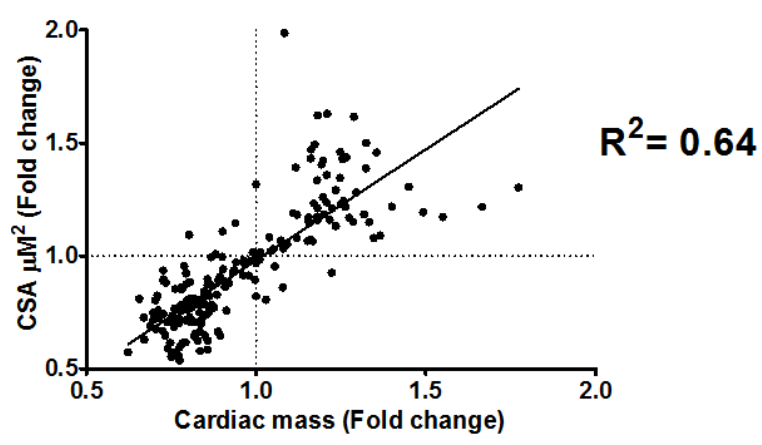

Supplemental figure 3. Correlation of fold change induced by the experimental condition between OCPS of hypertrophy cardiac mass and cardiomyocyte cross-sectional area (CSA). 
Supplemental table 4. Articles with general conclusions that show opposite directions for the pathophysiological processes of cardiac hypertrophy, fibrosis and inflammation

\begin{tabular}{lll}
\hline General conclusion & Author and year & PubMed ID \\
\hline Hypertrophy $\uparrow$ Fibrosis $\downarrow$ & Cai et al., 2010 & 20542874 \\
Hypertrophy $\downarrow$ Fibrosis $\uparrow$ & Honsho et al., 2009 & 19834007 \\
& Kazakov et al., 2012 & 22106415 \\
Hypertrophy $\uparrow$ Inflammation $\downarrow$ & Frolova et al., 2012 & 22362893 \\
& Cai et al., 2010 & 20542874 \\
& Ares-Carrasco, 2009 & 19820199 \\
Hypertrophy $\downarrow$ Inflammation $\uparrow$ & Liu et al., 2008 & 18490734 \\
Inflammation $\uparrow$ Fibrosis $\downarrow$ & Liu et al., 2008 & 18490734 \\
Inflammation $\downarrow$ Fibrosis $\uparrow$ & Frolova et al., 2012 & 22362893 \\
\hline
\end{tabular}

\section{References}

1. Bui AL, Horwich TB and Fonarow GC. Epidemiology and risk profile of heart failure. Nature reviews Cardiology. 2011;8:30-41.

2. Heineke J and Molkentin JD. Regulation of cardiac hypertrophy by intracellular signalling pathways. Nat Rev Mol Cell Biol. 2006; 7:589-600.

3. Weber KT, Sun Y, Guarda E, Katwa LC, Ratajska A, Cleutjens JP and Zhou G. Myocardial fibrosis in hypertensive heart disease: an overview of potential regulatory mechanisms. Eur Heart J. 1995;16 Suppl C:24-8.

4. Heymans S, Hirsch E, Anker SD, Aukrust $P$, Balligand JL, Cohen-Tervaert JW, Drexler H, Filippatos G, Felix SB, Gullestad L, Hilfiker-Kleiner D, Janssens S, Latini R, Neubauer G, Paulus WJ, Pieske B, Ponikowski P, Schroen B, Schultheiss HP, Tschope C, Van Bilsen M, Zannad F, McMurray J and Shah AM. Inflammation as a therapeutic target in heart failure? A scientific statement from the Translational Research Committee of the Heart Failure Association of the European Society of Cardiology. Eur J Heart Fail. 2009;11:119-29.

5. Frieler RA and Mortensen RM. Immune cell and other noncardiomyocyte regulation of cardiac hypertrophy and remodeling. Circulation. 2015;131:1019-30.

6. Nicoletti A and Michel JB. Cardiac fibrosis and inflammation: interaction with hemodynamic and hormonal factors. Cardiovasc Res. 1999;41:532-43.

7. Kuwahara F, Kai H, Tokuda K, Takeya M, Takeshita A, Egashira K and Imaizumi T. Hypertensive myocardial fibrosis and diastolic dysfunction: another model of inflammation? Hypertension. 2004;43:739-45.

8. Trachet B, Fraga-Silva RA, Jacquet PA, Stergiopulos N and Segers P. Incidence, severity, mortality, and confounding factors for dissecting AAA detection in angiotensin II-infused mice: a meta-analysis. Cardiovasc Res. 2015;108:159-70.

9. van der Spoel TI, Jansen of Lorkeers SJ, Agostoni P, van Belle E, Gyongyosi M, Sluijter JP, Cramer MJ, Doevendans PA and Chamuleau SA. Human relevance of pre-clinical studies in stem cell therapy: systematic review and meta-analysis of large animal models of ischaemic heart disease. Cardiovasc Res. 2011;91:649-58.

10. McCann SK, Irvine C, Mead GE, Sena ES, Currie GL, Egan KE, Macleod MR and Howells DW. Efficacy of antidepressants in animal models of ischemic stroke: a systematic review and meta-analysis. Stroke. 2014;45:3055-63.

11. Patten RD and Hall-Porter MR. Small animal models of heart failure: development of novel therapies, past and present. Circulation Heart failure. 2009;2:138-44.

12. Ikeda S, He A, Kong SW, Lu J, Bejar R, Bodyak N, Lee KH, Ma Q, Kang PM, Golub TR and Pu WT. MicroRNA1 negatively regulates expression of the hypertrophy-associated calmodulin and Mef2a genes. Mol Cell Biol. 2009;29:2193-204.

13. van Rooij E, Sutherland LB, Thatcher JE, DiMaio JM, Naseem RH, Marshall WS, Hill JA and Olson EN. Dysregulation of microRNAs after myocardial infarction reveals a role of miR-29 in cardiac fibrosis. Proc Natl Acad Sci U S A. 2008;105:13027-32.

14. Corsten MF, Papageorgiou A, Verhesen W, Carai P, Lindow M, Obad S, Summer G, Coort SL, Hazebroek M, van Leeuwen R, Gijbels MJ, Wijnands E, Biessen EA, De Winther MP, Stassen FR, Carmeliet P, Kauppinen S, Schroen B and Heymans S. MicroRNA profiling identifies microRNA-155 as an adverse mediator of cardiac injury and dysfunction during acute viral myocarditis. Circulation research. 2012;111:415-25.

15. Chou CH, Chang NW, Shrestha S, Hsu SD, Lin YL, Lee WH, Yang CD, Hong HC, Wei TY, Tu SJ, Tsai TR, Ho SY, Jian TY, Wu HY, Chen PR, Lin NC, Huang HT, Yang TL, Pai CY, Tai CS, Chen WL, Huang CY, Liu CC, Weng SL, 
Liao KW, Hsu WL and Huang HD. miRTarBase 2016: updates to the experimentally validated miRNA-target interactions database. Nucleic Acids Res. 2015.

16. Agarwal V, Bell GW, Nam JW and Bartel DP. Predicting effective microRNA target sites in mammalian mRNAs. Elife. 2015;4.

17. Wong $\mathrm{N}$ and Wang $\mathrm{X}$. miRDB: an online resource for microRNA target prediction and functional annotations. Nucleic Acids Res. 2015;43:D146-52.

18. Paraskevopoulou MD, Georgakilas G, Kostoulas N, Vlachos IS, Vergoulis T, Reczko M, Filippidis C, Dalamagas $T$ and Hatzigeorgiou AG. DIANA-microT web server v5.0: service integration into miRNA functional analysis workflows. Nucleic Acids Res. 2013;41:W169-73.

19. Summer G, Kelder T, Ono K, Radonjic M, Heymans S and Demchak B. cyNeo4j: connecting Neo4j and Cytoscape. Bioinformatics. 2015;31:3868-9.

20. Shannon P, Markiel A, Ozier O, Baliga NS, Wang JT, Ramage D, Amin N, Schwikowski B and Ideker T. Cytoscape: a software environment for integrated models of biomolecular interaction networks. Genome Res. 2003;13:2498-504.

21. Frolova EG, Sopko N, Blech L, Popovic ZB, Li J, Vasanji A, Drumm C, Krukovets I, Jain MK, Penn MS, Plow $\mathrm{EF}$ and Stenina OI. Thrombospondin-4 regulates fibrosis and remodeling of the myocardium in response to pressure overload. FASEB J. 2012;26:2363-73.

22. Cai WF, Zhang XW, Yan HM, Ma YG, Wang XX, Yan J, Xin BM, Lv XX, Wang QQ, Wang ZY, Yang HZ and Hu ZW. Intracellular or extracellular heat shock protein 70 differentially regulates cardiac remodelling in pressure overload mice. Cardiovasc Res. 2010;88:140-9.

23. Ares-Carrasco S, Picatoste B, Benito-Martin A, Zubiri I, Sanz AB, Sanchez-Nino MD, Ortiz A, Egido J, Tunon $\mathrm{J}$ and Lorenzo O. Myocardial fibrosis and apoptosis, but not inflammation, are present in long-term experimental diabetes. Am J Physiol Heart Circ Physiol. 2009;297:H2109-19.

24. Liu YY, Cai WF, Yang HZ, Cui B, Chen ZR, Liu HZ, Yan J, Jin W, Yan HM, Xin BM, Yuan B, Hua F and Hu ZW. Bacillus Calmette-Guerin and TLR4 agonist prevent cardiovascular hypertrophy and fibrosis by regulating immune microenvironment. J Immunol. 2008;180:7349-57.

25. Unger T. The role of the renin-angiotensin system in the development of cardiovascular disease. Am $\mathrm{J}$ Cardiol. 2002;89:3A-9A; discussion 10A.

26. Watkins SJ, Borthwick GM, Oakenfull R, Robson A and Arthur HM. Angiotensin II-induced cardiomyocyte hypertrophy in vitro is TAK1-dependent and Smad2/3-independent. Hypertens Res. 2012;35:393-8.

27. McMaster WG, Kirabo A, Madhur MS and Harrison DG. Inflammation, immunity, and hypertensive endorgan damage. Circ Res. 2015;116:1022-33.

28. Gullestad L, Ueland T, Vinge LE, Finsen A, Yndestad A and Aukrust P. Inflammatory cytokines in heart failure: mediators and markers. Cardiology. 2012;122:23-35.

29. Mann DL. Inflammatory mediators and the failing heart: past, present, and the foreseeable future. Circ Res. 2002;91:988-98.

30. Sun M, Chen M, Dawood F, Zurawska U, Li JY, Parker T, Kassiri Z, Kirshenbaum LA, Arnold M, Khokha R and Liu PP. Tumor necrosis factor-alpha mediates cardiac remodeling and ventricular dysfunction after pressure overload state. Circulation. 2007;115:1398-407.

31. Wang Y, Li Y, Wu Y, Jia L, Wang J, Xie B, Hui M and Du J. 5TNF-alpha and IL-1beta neutralization ameliorates angiotensin II-induced cardiac damage in male mice. Endocrinology. 2014;155:2677-87.

32. Coles B, Fielding CA, Rose-John S, Scheller J, Jones SA and O'Donnell VB. Classic interleukin-6 receptor signaling and interleukin-6 trans-signaling differentially control angiotensin II-dependent hypertension, cardiac signal transducer and activator of transcription-3 activation, and vascular hypertrophy in vivo. The American journal of pathology. 2007;171:315-25.

33. Lai NC, Gao MH, Tang E, Tang R, Guo T, Dalton ND, Deng A and Tang T. Pressure overload-induced cardiac remodeling and dysfunction in the absence of interleukin 6 in mice. Lab Invest. 2012;92:1518-26.

34. Ma F, Li Y, Jia L, Han Y, Cheng J, Li H, Qi Y and Du J. Macrophage-stimulated cardiac fibroblast production of IL-6 is essential for TGF beta/Smad activation and cardiac fibrosis induced by angiotensin II. PLoS One. 2012;7:e35144.

35. Garcia AG, Wilson RM, Heo J, Murthy NR, Baid S, Ouchi N and Sam F. Interferon-gamma ablation exacerbates myocardial hypertrophy in diastolic heart failure. Am J Physiol Heart Circ Physiol. 2012;303:H587-96.

36. Marko L, Kvakan H, Park JK, Qadri F, Spallek B, Binger KJ, Bowman EP, Kleinewietfeld M, Fokuhl V, Dechend $\mathrm{R}$ and Muller DN. Interferon-gamma signaling inhibition ameliorates angiotensin II-induced cardiac damage. Hypertension. 2012;60:1430-6.

37. Honsho S, Nishikawa S, Amano K, Zen K, Adachi Y, Kishita E, Matsui A, Katsume A, Yamaguchi S, Nishikawa K, Isoda K, Riches DW, Matoba S, Okigaki M and Matsubara H. Pressure-mediated hypertrophy and 
mechanical stretch induces IL-1 release and subsequent IGF-1 generation to maintain compensative hypertrophy by affecting Akt and JNK pathways. Circ Res. 2009;105:1149-58.

38. Jiang DS, Li L, Huang L, Gong J, Xia H, Liu X, Wan N, Wei X, Zhu X, Chen Y, Chen X, Zhang XD and Li H. Interferon regulatory factor 1 is required for cardiac remodeling in response to pressure overload. Hypertension. 2014;64:77-86.

39. Jiang DS, Bian ZY, Zhang Y, Zhang SM, Liu Y, Zhang R, Chen Y, Yang Q, Zhang XD, Fan GC and Li H. Role of interferon regulatory factor 4 in the regulation of pathological cardiac hypertrophy. Hypertension. 2013;61:1193-202.

40. Jiang DS, Luo YX, Zhang R, Zhang XD, Chen HZ, Zhang Y, Chen K, Zhang SM, Fan GC, Liu PP, Liu DP and Li H. Interferon regulatory factor 9 protects against cardiac hypertrophy by targeting myocardin. Hypertension. 2014;63:119-27.

41. Li Y, Dalli J, Chiang N, Baron RM, Quintana C and Serhan CN. Plasticity of leukocytic exudates in resolving acute inflammation is regulated by MicroRNA and proresolving mediators. Immunity. 2013;39:885-98.

42. Wang D, Zhang $H$, Li M, Frid MG, Flockton AR, McKeon BA, Yeager ME, Fini MA, Morrell NW, Pullamsetti SS, Velegala S, Seeger W, McKinsey TA, Sucharov CC and Stenmark KR. MicroRNA-124 controls the proliferative, migratory, and inflammatory phenotype of pulmonary vascular fibroblasts. Circ Res. 2014;114:67-78.

43. Kang K, Peng X, Zhang X, Wang Y, Zhang L, Gao L, Weng T, Zhang H, Ramchandran R, Raj JU, Gou D and Liu L. MicroRNA-124 suppresses the transactivation of nuclear factor of activated T cells by targeting multiple genes and inhibits the proliferation of pulmonary artery smooth muscle cells. J Biol Chem. 2013;288:25414-27.

44. van Almen GC, Verhesen $W$, van Leeuwen RE, van de Vrie $M$, Eurlings $C$, Schellings $M W$, Swinnen $M$, Cleutjens JP, van Zandvoort MA, Heymans S and Schroen B. MicroRNA-18 and microRNA-19 regulate CTGF and TSP-1 expression in age-related heart failure. Aging Cell. 2011;10:769-79.

45. Zhu X, Wang H, Liu F, Chen L, Luo W, Su P, Li W, Yu L, Yang X and Cai J. Identification of micro-RNA networks in end-stage heart failure because of dilated cardiomyopathy. J Cell Mol Med. 2013;17:1173-87.

46. van Hout GP, Jansen Of Lorkeers SJ, Wever KE, Sena ES, Kouwenberg LH, van Solinge WW, Macleod MR, Doevendans PA, Pasterkamp G, Chamuleau SA and Hoefer IE. Translational failure of anti-inflammatory compounds for myocardial infarction: a meta-analysis of large animal models. Cardiovasc Res. 2015.

47. Sena ES, van der Worp HB, Bath PM, Howells DW and Macleod MR. Publication bias in reports of animal stroke studies leads to major overstatement of efficacy. PLoS Biol. 2010;8:e1000344.

48. Korevaar DA, Hooft $L$ and ter Riet G. Systematic reviews and meta-analyses of preclinical studies: publication bias in laboratory animal experiments. Lab Anim. 2011;45:225-30.

49. Bogue MA and Grubb SC. The Mouse Phenome Project. Genetica. 2004;122:71-4.

50. Xia Y, Lee K, Li N, Corbett D, Mendoza L and Frangogiannis NG. Characterization of the inflammatory and fibrotic response in a mouse model of cardiac pressure overload. Histochemistry and cell biology. 2009;131:471-81.

51. Rizzi E, Ceron CS, Guimaraes DA, Prado CM, Rossi MA, Gerlach RF and Tanus-Santos JE. Temporal changes in cardiac matrix metalloproteinase activity, oxidative stress, and TGF-beta in renovascular hypertensioninduced cardiac hypertrophy. Exp Mol Pathol. 2013;94:1-9.

52. Stewart JA, Jr., Massey EP, Fix C, Zhu J, Goldsmith EC and Carver W. Temporal alterations in cardiac fibroblast function following induction of pressure overload. Cell and tissue research. 2010;340:117-26.

53. Van Linthout S, Miteva $\mathrm{K}$ and Tschope C. Crosstalk between fibroblasts and inflammatory cells. Cardiovasc Res. 2014;102:258-69.

54. Mohammed SF, Storlie JR, Oehler EA, Bowen LA, Korinek J, Lam CS, Simari RD, Burnett JC, Jr. and Redfield MM. Variable phenotype in murine transverse aortic constriction. Cardiovasc Pathol. 2012;21:188-98.

55. Peng H, Yang XP, Carretero OA, Nakagawa P, D'Ambrosio M, Leung P, Xu J, Peterson EL, Gonzalez GE, Harding $P$ and Rhaleb NE. Angiotensin II-induced dilated cardiomyopathy in Balb/c but not C57BL/6J mice. Exp Physiol. 2011;96:756-64.

56. Garcia-Menendez L, Karamanlidis G, Kolwicz S and Tian R. Substrain specific response to cardiac pressure overload in C57BL/6 mice. Am J Physiol Heart Circ Physiol. 2013;305:H397-402.

57. Kuroki MT, Fink GD and Osborn JW. Comparison of arterial pressure and plasma ANG II responses to three methods of subcutaneous ANG II administration. Am J Physiol Heart Circ Physiol. 2014;307:H670-9. 


\section{Supplemental File 1. Overview of all included studies.}

* article contains multiple studies. Pm ID = PubMed ID. Gene $=$ The Ensembl name of the manipulated gene and the direction of manipulation: $\downarrow$ Down (e.g. knockout) $\uparrow=$ up (e.g. transgenic). General conclusions regarding cardiac hypertrophy $(H)$, fibrosis (F) and inflammation (I) are indicated as follows: reduced ( $\downarrow)$, increased ( $\uparrow)$ or no significant change (-) ND = No data / Not determined.

\begin{tabular}{|c|c|c|c|c|c|c|c|c|}
\hline First Author & Year & Title & Pm ID & $\mathrm{s}$ & gene & $\mathrm{H}$ & $\mathrm{F}$ & 1 \\
\hline Muller & 2000 & $\begin{array}{l}\text { NF-kappaB inhibition ameliorates angiotensin II-induced inflammatory damage in } \\
\text { rats. }\end{array}$ & 10642297 & $\mathrm{R}$ & & $\downarrow$ & ND & $\downarrow$ \\
\hline Akishita* & 2000 & $\begin{array}{l}\text { Inhibitory Effect of Angiotensin II Type } 2 \text { Receptor on Coronary Arterial Remodeling } \\
\text { after Aortic Banding in Mice }\end{array}$ & 11015348 & M & Agtr2 $\downarrow$ & $\uparrow$ & $\uparrow$ & ND \\
\hline Akishita* & 2000 & $\begin{array}{l}\text { Inhibitory Effect of Angiotensin II Type } 2 \text { Receptor on Coronary Arterial Remodeling } \\
\text { after Aortic Banding in Mice }\end{array}$ & 11015348 & M & Agtr2 $\downarrow$ & - & $\uparrow$ & ND \\
\hline Koyanagi & 2000 & $\begin{array}{l}\text { Role of monocyte chemoattractant protein-1 in cardiovascular remodeling induced } \\
\text { by chronic blockade of nitric oxide synthesis. }\end{array}$ & 11056100 & $\mathrm{R}$ & $C c / 2 \downarrow$ & ND & - & $\downarrow$ \\
\hline Sakata & 2000 & $\begin{array}{l}\text { Calcineurin Inhibitor Attenuates Left Ventricular hypertrophy, Leading to } \\
\text { Prevention of Heart Failure in Hypertensive Rats }\end{array}$ & 11056104 & $\mathrm{R}$ & & $\downarrow$ & - & ND \\
\hline Uozumi & 2001 & gp130 plays a critical role in pressure overload-induced cardiac hypertrophy. & 11262406 & M & $\| 6 s t \downarrow$ & $\downarrow$ & ND & ND \\
\hline Knowles & 2001 & $\begin{array}{l}\text { Pressure-independent enhancement of cardiac hypertrophy in natriuretic peptide } \\
\text { receptor A-deficient mice. }\end{array}$ & 11306601 & M & $N p r 1 \downarrow$ & $\uparrow$ & $\uparrow$ & ND \\
\hline Ichihara & 2001 & $\begin{array}{l}\text { Angiotensin II Type } 2 \text { Receptor Is Essential for Left Ventricular Hypertrophy and } \\
\text { Cardiac Fibrosis in Chronic Angiotensin II-Induced Hypertension }\end{array}$ & 11457756 & M & Agtr2 $\downarrow$ & $\downarrow$ & $\downarrow$ & ND \\
\hline Rapacciuolo & 2001 & $\begin{array}{l}\text { Important role of endogenous norepinephrine and epinephrine in the development } \\
\text { of in vivo pressure-overload cardiac hypertrophy. }\end{array}$ & 11527648 & M & $D b h \downarrow$ & $\downarrow$ & ND & ND \\
\hline Eickels* & 2001 & 17b-Estradiol Attenuates the Development of Pressure-Overload Hypertrophy & 11560859 & M & & $\downarrow$ & - & ND \\
\hline Eickels* & 2001 & 17b-Estradiol Attenuates the Development of Pressure-Overload Hypertrophy & 11560859 & M & & $\downarrow$ & ND & ND \\
\hline Moser* & 2002 & $\begin{array}{l}\text { Predominant activation of endothelin-dependent cardiac hypertrophy by } \\
\text { norepinephrine in rat left ventricle. }\end{array}$ & 11959681 & $R$ & & - & ND & ND \\
\hline Moser* & 2002 & $\begin{array}{l}\text { Predominant activation of endothelin-dependent cardiac hypertrophy by } \\
\text { norepinephrine in rat left ventricle. }\end{array}$ & 11959681 & $\mathrm{R}$ & & - & ND & ND \\
\hline Baumgarten* & 2002 & $\begin{array}{l}\text { Load-Dependeent and -Independent Regulation of proinflammatory Cytokine and } \\
\text { Cytokine receptor Gene Expression in the Adult mammalian heart }\end{array}$ & 11994254 & M & & ND & ND & ND \\
\hline Baumgarten* & 2002 & $\begin{array}{l}\text { Load-Dependeent and -Independent Regulation of proinflammatory Cytokine and } \\
\text { Cytokine receptor Gene Expression in the Adult mammalian heart }\end{array}$ & 11994254 & M & & ND & ND & - \\
\hline Baumgarten* & 2002 & $\begin{array}{l}\text { Load-Dependeent and -Independent Regulation of proinflammatory Cytokine and } \\
\text { Cytokine receptor Gene Expression in the Adult mammalian heart }\end{array}$ & 11994254 & $\mathrm{R}$ & & ND & ND & ND \\
\hline Jeron & 2002 & $\begin{array}{l}\text { Systemic immunosuppression fails to suppress cardiac cytokine induction in } \\
\text { pressure overload hypertrophy in rats. }\end{array}$ & 11999344 & $\mathrm{R}$ & & - & ND & $\downarrow$ \\
\hline Kuwahara & 2002 & $\begin{array}{l}\text { Transforming growth factor-beta function blocking prevents myocardial fibrosis } \\
\text { and diastolic dysfunction in pressure-overloaded rats. }\end{array}$ & 12093782 & $\mathrm{R}$ & $T g f b 1 \downarrow$ & - & $\downarrow$ & ND \\
\hline Sadoshima & 2002 & $\begin{array}{l}\text { The MEKK1-JNK pathway plays a protective role in pressure overload but does not } \\
\text { mediate cardiac hypertrophy. }\end{array}$ & 12122119 & M & Map3k1 $\downarrow$ & - & ND & $\uparrow$ \\
\hline Nakayama & 2003 & $\begin{array}{l}\text { Cardiac-specific overexpression of a high Ca2+ affinity mutant of SERCA2a } \\
\text { attenuates in vivo pressure overload cardiac hypertrophy. }\end{array}$ & 12424227 & M & Atp2a1 个 & $\downarrow$ & ND & ND \\
\hline Cingolani* & 2003 & Regression of hypertensive myocardial fibrosis by $\mathrm{Na}(+) / \mathrm{H}(+)$ exchange inhibition. & 12574110 & $\mathrm{R}$ & & $\downarrow$ & $\downarrow$ & ND \\
\hline Cingolani* & 2003 & Regression of hypertensive myocardial fibrosis by $\mathrm{Na}(+) / \mathrm{H}(+)$ exchange inhibition. & 12574110 & $\mathrm{R}$ & & $\downarrow$ & $\downarrow$ & ND \\
\hline Cingolani* & 2003 & Regression of hypertensive myocardial fibrosis by $\mathrm{Na}(+) / \mathrm{H}(+)$ exchange inhibition. & 12574110 & $\mathrm{R}$ & & $\downarrow$ & - & ND \\
\hline Kuwahara & 2003 & Roles of intercellular adhesion molecule- 1 in hypertensive cardiac remodeling. & 12624002 & $\mathrm{R}$ & $\operatorname{Icam} 1 \downarrow$ & - & $\downarrow$ & $\downarrow$ \\
\hline Zhang & 2003 & $\begin{array}{l}\text { The role of the Grb2-p38 MAPK signaling pathway in cardiac hypertrophy and } \\
\text { fibrosis. }\end{array}$ & 12639989 & M & Grb2 $\downarrow$ & $\downarrow$ & $\downarrow$ & ND \\
\hline $\mathrm{Hu}$ & 2003 & $\begin{array}{l}\text { Minimally invasive aortic banding in mice: effects of altered cardiomyocyte insulin } \\
\text { signaling during pressure overload. }\end{array}$ & 12738623 & M & Insr $\downarrow$ & - & $\uparrow$ & ND \\
\hline Wang* & 2003 & $\begin{array}{l}\text { Effects of pressure overload on extracellular matrix expression in the heart of the } \\
\text { atrial natriuretic peptide-null mouse. }\end{array}$ & 12756220 & M & Nppa $\downarrow$ & - & - & ND \\
\hline Wang* & 2003 & $\begin{array}{l}\text { Effects of pressure overload on extracellular matrix expression in the heart of the } \\
\text { atrial natriuretic peptide-null mouse. }\end{array}$ & 12756220 & M & $N p p a \downarrow$ & $\uparrow$ & $\uparrow$ & ND \\
\hline Wang* & 2003 & $\begin{array}{l}\text { Effects of pressure overload on extracellular matrix expression in the heart of the } \\
\text { atrial natriuretic peptide-null mouse. }\end{array}$ & 12756220 & M & Nppa $\downarrow$ & $\uparrow$ & $\uparrow$ & ND \\
\hline Okumura & 2003 & $\begin{array}{l}\text { Disruption of type } 5 \text { adenylyl cyclase gene preserves cardiac function against } \\
\text { pressure overload. }\end{array}$ & 12904575 & M & $\operatorname{Adcy} 5 \downarrow$ & - & ND & ND \\
\hline Takahashi & 2003 & $\begin{array}{l}\text { Angiotensin II-induced ventricular hypertrophy and extracellular signal-regulated } \\
\text { kinase activation are suppressed in mice overexpressing brain natriuretic peptide in } \\
\text { circulation. }\end{array}$ & 14621189 & M & $N p p b \uparrow$ & $\downarrow$ & $\downarrow$ & ND \\
\hline Ichinose & 2003 & $\begin{array}{l}\text { Pressure overload-induced LV hypertrophy and dysfunction in mice are } \\
\text { exacerbated by congenital NOS3 deficiency. }\end{array}$ & 14644766 & M & Nos3 $\downarrow$ & $\uparrow$ & $\uparrow$ & ND \\
\hline Diep & 2004 & $\begin{array}{l}\text { PPARa activator fenofibrate inhibits myocardial inflammation and fibrosis in } \\
\text { angiotensin II-infused rats }\end{array}$ & 14871557 & $\mathrm{R}$ & & - & $\downarrow$ & $\downarrow$ \\
\hline Kuwahara & 2004 & $\begin{array}{l}\text { Hypertensive myocardial fibrosis and diastolic dysfunction: another model of } \\
\text { inflammation? }\end{array}$ & 14967845 & $\mathrm{R}$ & $C \mathrm{Cl} / 2 \downarrow$ & - & $\downarrow$ & $\downarrow$ \\
\hline Rasoul & 2004 & $\begin{array}{l}\text { Antifibrotic effect of Ac-SDKP and angiotensin-converting enzyme inhibition in } \\
\text { hypertension. }\end{array}$ & 15076166 & $\mathrm{R}$ & & - & $\downarrow$ & $\downarrow$ \\
\hline Chen & 2004 & $\begin{array}{l}\text { Heat shock treatment suppresses angiotensin II-induced activation of NF-kappaB } \\
\text { pathway and heart inflammation: a role for IKK depletion by heat shock? }\end{array}$ & 15087290 & $\mathrm{R}$ & & ND & $\downarrow$ & $\downarrow$ \\
\hline Hikoso & 2004 & $\begin{array}{l}\text { Pressure overload induces cardiac dysfunction and dilation in signal transducer and } \\
\text { activator of transcription 6-deficient mice. }\end{array}$ & 15492321 & M & Stat6 $\downarrow$ & - & $\uparrow$ & $\uparrow$ \\
\hline Gao & 2005 & Regression of pressure overload-induced left ventricular hypertrophy in mice. & 15665058 & M & & ND & ND & ND \\
\hline
\end{tabular}


Mineralocorticoid receptor inhibition ameliorates the transition to myocardia failure and decreases oxidative stress and inflammation in mice with chronic pressure overload.

Kawano* 2005

Kawano* 2006

$\mathrm{Ha}$

Hara

Kassiri

(1)

$\mathrm{Ha}$

Peng

Nagata

O'Connell

Kong*

Kong*

Gao

Empel

Franco

2006

Bisping

Sakamoto

2006

Ren

Harada

Sun

Duhaney

Buys

Sanada*

Sanada*

Nishioka

Oka

Coles*

Coles*

2007

Freeling*

2008

Freeling*

2008

Ruiz-Hurtado

2007

Müller

2008

Sharma

Smeets

Pillai

Kiriazis

Pfor-kappaB ameliorates myocardial hypertrophy in response to chronic infusion of angiotensin II.

Blockade of NF-kappaB ameliorates myocardial hypertrophy in response to chronic infusion of angiotensin II.

Reduced cardiac hypertrophy in toll-like receptor 4-deficient mice following pressure overload

Augmented cardiac hypertrophy in response to pressure overload in mice lacking the prostaglandin 12 receptor.

Combination of tumor necrosis factor-alpha ablation and matrix metalloproteinase inhibition prevents heart failure after pressure overload in tissue inhibitor of metalloproteinase-3 knock-out mice.

Blockade of MyD88 attenuates cardiac hypertrophy and decreases cardiac myocyte apoptosis in pressure overload-induced cardiac hypertrophy in vivo.

Angiotensin-converting enzyme inhibitors: a new mechanism of action.

Mineralocorticoid receptor antagonism attenuates cardiac hypertrophy and failure

in low-aldosterone hypertensive rats.

Alpha1-adrenergic receptors prevent a maladaptive cardiac response to pressure overload.

Suppression of class I and II histone deacetylases blunts pressure-overload cardiac hypertrophy.

Suppression of class I and II histone deacetylases blunts pressure-overload cardiac hypertrophy.

Inhibition of mTOR reduces chronic pressure-overload cardiac hypertrophy and fibrosis.

EUK-8, a Superoxide Dismutase and Catalase Mimetic, Reduces Cardiac Oxidative Stress and Ameliorates Pressure Overload-Induced Heart Failure in the Harlequin Mouse Mutant

Eplerenone prevents adverse cardiac remodelling induced by pressure overload in atrial natriuretic peptide-null mice.

Gata4 is required for maintenance of postnatal cardiac function and protection from pressure overload-induced heart failure.

Upregulation of heat shock transcription factor 1 plays a critical role in adaptive cardiac hypertrophy.

Beta3 integrin deficiency promotes cardiac hypertrophy and inflammation.

Diacylglycerol kinase zeta attenuates pressure overload-induced cardiac

hypertrophy.

Tumor necrosis factor-alpha mediates cardiac remodeling and ventricular dysfunction after pressure overload state

Peroxisome proliferator-activated receptor alpha-independent actions of fenofibrate exacerbates left ventricular dilation and fibrosis in chronic pressure overload.

Cardiomyocyte-restricted restoration of nitric oxide synthase 3 attenuates left ventricular remodeling after chronic pressure overload.

IL-33 and ST2 comprise a critical biomechanically induced and cardioprotective signaling system.

IL-33 and ST2 comprise a critical biomechanically induced and cardioprotective signaling system.

Eplerenone attenuates myocardial fibrosis in the angiotensin II-induced

hypertensive mouse: involvement of tenascin- $\mathrm{C}$ induced by aldosterone-mediated inflammation.

Genetic manipulation of periostin expression reveals a role in cardiac hypertrophy and ventricular remodeling.

Classic interleukin- 6 receptor signaling and interleukin- 6 trans-signaling

differentially control angiotensin II-dependent hypertension, cardiac signal

transducer and activator of transcription-3 activation, and vascular hypertrophy in vivo.

Classic interleukin- 6 receptor signaling and interleukin- 6 trans-signalin differentially control angiotensin II-dependent hypertension, cardiac signal transducer and activator of transcription-3 activation, and vascular hypertrophy in vivo.

Neostigmine and pilocarpine attenuated tumour necrosis factor alpha expression and cardiac hypertrophy in the heart with pressure overload.

Neostigmine and pilocarpine attenuated tumour necrosis factor alpha expression and cardiac hypertrophy in the heart with pressure overload.

LA419, a novel nitric oxide donor, prevents pathological cardiac remodeling in pressure-overloaded rats via endothelial nitric oxide synthase pathway regulation. Pressure-induced cardiac overload induces upregulation of endothelial and myocardial progenitor cells.

Novel anti-inflammatory mechanisms of N-Acetyl-Ser-Asp-Lys-Pro in hypertensioninduced target organ damage.

Cardiac hypertrophy is enhanced in PPAR alpha-/- mice in response to chronic pressure overload.

Activation of SIRT1, a class III histone deacetylase, contributes to fructose feedingmediated induction of the alpha-myosin heavy chain expression.

2008

\section{$15687129 \quad \mathrm{M}$}

$15921667 \quad M \quad N f k b 1 \downarrow \quad-\quad N D \quad N D$

$15921667 \quad M \quad N f k b 1 \downarrow \quad \downarrow \quad N D \quad-$

$15967420 \quad \mathrm{M} \quad$ TIr4 $\downarrow \quad \downarrow \quad-\quad \downarrow$

15983244 M Ptgir $\downarrow \quad \uparrow \quad \uparrow \quad$ ND

16037568 M Timp3 $\downarrow \quad \uparrow \quad \uparrow \quad$ ND

$16199478 \quad R \quad M y d 88 \downarrow \quad \downarrow \quad \downarrow \quad \downarrow$

$16216963 \mathrm{R} \quad-\quad \downarrow \quad \downarrow$

$16505208 \mathrm{R} \quad \downarrow \quad \downarrow \quad \downarrow$

16585965 M Adra1a $\downarrow, \quad-\quad \uparrow \quad$ ND

Adra1b $\downarrow$

16735673 M Hist1h3a $\downarrow \quad \downarrow \quad-\quad$ ND

16735673 M Hist1h3a $\downarrow \quad \downarrow \quad$ ND ND

$16877971 \quad \mathrm{M} \quad \downarrow \quad \downarrow \quad$ ND

$\begin{array}{llllll}16904556 & \text { M Aifm } 1 \downarrow & \uparrow & \uparrow & \text { ND }\end{array}$

16922805 M Nppa $\downarrow \quad \uparrow \quad \uparrow \quad$ ND

16983087 M Gata4 $\downarrow \quad \uparrow \quad \uparrow \quad$ ND

$17095722 \mathrm{M} \quad H S F 1 \uparrow \quad \downarrow \quad \downarrow \quad$ ND

$17184791 \mathrm{M} \quad \operatorname{ltg} 3 \downarrow \quad \uparrow \quad$ ND $\quad \uparrow$

$17251681 \quad \mathrm{M} \quad \operatorname{Dgkz} \uparrow \quad \downarrow \quad \downarrow \quad \downarrow$

17353445 M Tnf $\downarrow, / 11 b \quad \downarrow \quad \downarrow \quad \downarrow$

$17353509 \mathrm{M} \quad \uparrow \quad \uparrow \quad$ ND

17416602 M Nos3 $\downarrow \quad \uparrow \quad-\quad$ ND

17492053 M Tmed1 $\downarrow \quad \uparrow \quad \uparrow \quad \uparrow$

$17492053 \quad \mathrm{M} \quad$ /133个 $\uparrow \quad \downarrow \quad \downarrow \quad \downarrow$

17513943 M ND $\downarrow \downarrow \downarrow$

17569887 M Postn $\downarrow \quad \downarrow \quad \downarrow \quad$ ND

$17591976 \quad \mathrm{M} \quad 1 / 6 \downarrow \quad \downarrow \quad$ ND ND

$17591976 \quad \mathrm{M} \quad$ ll6st 个

$17872965 \quad R$

17872965

$17984372 \quad \mathrm{R}$

$18006457 \quad M$

$18178715 \quad M$

$18187461 \quad M$

Ppara $\downarrow$

$\downarrow \quad$ ND $\quad \downarrow$

$\downarrow \quad$ ND $\downarrow$

$\downarrow \quad \downarrow \quad N D$

$\downarrow \quad$ ND ND

$18192211 \quad M$

18193078

M




\begin{tabular}{|c|c|c|c|c|c|c|c|c|}
\hline $\begin{array}{l}\text { Nonaka- } \\
\text { Sarukawa }\end{array}$ & 2008 & $\begin{array}{l}\text { Adeno-associated virus vector-mediated systemic interleukin-10 expression } \\
\text { ameliorates hypertensive organ damage in Dahl salt-sensitive rats. }\end{array}$ & 18205252 & $\mathrm{R}$ & $1110 \uparrow$ & $\downarrow$ & $\downarrow$ & ND \\
\hline Oudit & 2008 & $\begin{array}{l}\text { Loss of PTEN attenuates the development of pathological hypertrophy and heart } \\
\text { failure in response to biomechanical stress. }\end{array}$ & 18281373 & M & Pten $\downarrow$ & $\downarrow$ & $\downarrow$ & ND \\
\hline Xu & 2008 & $\begin{array}{l}\text { Ecto-5'-nucleotidase deficiency exacerbates pressure-overload-induced left } \\
\text { ventricular hypertrophy and dysfunction. }\end{array}$ & 18391093 & M & $N t 5 e \downarrow$ & $\uparrow$ & $\uparrow$ & $\uparrow$ \\
\hline Giampietri & 2008 & $\begin{array}{l}\text { c-Flip overexpression reduces cardiac hypertrophy in response to pressure } \\
\text { overload. }\end{array}$ & 18398344 & M & Cflar $\uparrow$ & $\downarrow$ & - & $\downarrow$ \\
\hline Mito & 2008 & $\begin{array}{l}\text { Myocardial protection against pressure overload in mice lacking Bach1, a } \\
\text { transcriptional repressor of heme oxygenase-1. }\end{array}$ & 18426999 & M & Bach1 $\downarrow$ & $\downarrow$ & $\downarrow$ & ND \\
\hline Moens & 2008 & $\begin{array}{l}\text { Reversal of cardiac hypertrophy and fibrosis from pressure overload by } \\
\text { tetrahydrobiopterin: efficacy of recoupling nitric oxide synthase as a therapeutic } \\
\text { strategy. }\end{array}$ & 18474817 & M & & $\downarrow$ & $\downarrow$ & ND \\
\hline Liu & 2008 & $\begin{array}{l}\text { Bacillus Calmette-Guérin and TLR4 agonist prevent cardiovascular hypertrophy and } \\
\text { fibrosis by regulating immune microenvironment. }\end{array}$ & 18490734 & M & & $\downarrow$ & $\downarrow$ & - \\
\hline Caglayan* & 2009 & $\begin{array}{l}\text { Differential Roles of Cardiomyocyte and Macrophage Peroxisome Proliferator- } \\
\text { Activated Receptor } \gamma \text { in Cardiac Fibrosis }\end{array}$ & 18511847 & M & Pparg $\downarrow$ & $\uparrow$ & - & $\uparrow$ \\
\hline Caglayan* & 2008 & $\begin{array}{l}\text { Differential Roles of Cardiomyocyte and Macrophage Peroxisome Proliferator- } \\
\text { Activated Receptor } \gamma \text { in Cardiac Fibrosis }\end{array}$ & 18511847 & M & Pparg $\downarrow$ & $\uparrow$ & $\uparrow$ & $\uparrow$ \\
\hline Foronjy & 2008 & $\begin{array}{l}\text { Transgenic expression of matrix metalloproteinase- } 1 \text { inhibits myocardial fibrosis } \\
\text { and prevents the transition to heart failure in a pressure overload mouse model. }\end{array}$ & 18633185 & M & Mmp1a 个 & $\downarrow$ & $\downarrow$ & ND \\
\hline Gallo & 2008 & $\begin{array}{l}\text { Inhibition of class I histone deacetylase with an apicidin derivative prevents cardiac } \\
\text { hypertrophy and failure. }\end{array}$ & 18697792 & M & & $\downarrow$ & $\downarrow$ & ND \\
\hline Copaja Soto & 2008 & $\begin{array}{l}\text { Early expression of monocyte chemoattractant protein-1 correlates with the onset } \\
\text { of isoproterenol-induced cardiac fibrosis in rats with distinct angiotensin- } \\
\text { converting enzyme polymorphism }\end{array}$ & 18957386 & $\mathrm{R}$ & & - & - & - \\
\hline Di Zhang & 2008 & $\begin{array}{l}\text { Cross-Talk Between Mineralocorticoid and Angiotensin II Signaling for Cardiac } \\
\text { Remodeling }\end{array}$ & 18981328 & M & $\begin{array}{l}\text { NR3C2-003 } \\
\uparrow\end{array}$ & $\uparrow$ & $\uparrow$ & - \\
\hline Xia* & 2008 & $\begin{array}{l}\text { Characterization of the inflammatory and fibrotic response in a mouse model of } \\
\text { cardiac pressure overload. }\end{array}$ & 19030868 & M & & ND & ND & ND \\
\hline Xia* & 2008 & $\begin{array}{l}\text { Characterization of the inflammatory and fibrotic response in a mouse model of } \\
\text { cardiac pressure overload. }\end{array}$ & 19030868 & M & & ND & ND & ND \\
\hline Xia* & 2008 & $\begin{array}{l}\text { Characterization of the inflammatory and fibrotic response in a mouse model of } \\
\text { cardiac pressure overload. }\end{array}$ & 19030868 & M & & ND & ND & ND \\
\hline Molina* & 2009 & $\begin{array}{l}\text { Improvement in hemodynamic performance, exercise capacity, inflammatory } \\
\text { profile, and left ventricular reverse remodeling after intracoronary delivery of } \\
\text { mesenchymal stem cells in an experimental model of pressure overload } \\
\text { hypertrophy. }\end{array}$ & 19065545 & $\mathrm{R}$ & & ND & ND & ND \\
\hline Molina* & 2009 & $\begin{array}{l}\text { Improvement in hemodynamic performance, exercise capacity, inflammatory } \\
\text { profile, and left ventricular reverse remodeling after intracoronary delivery of } \\
\text { mesenchymal stem cells in an experimental model of pressure overload } \\
\text { hypertrophy. }\end{array}$ & 19065545 & $\mathrm{R}$ & & ND & ND & ND \\
\hline Molina* & 2009 & $\begin{array}{l}\text { Improvement in hemodynamic performance, exercise capacity, inflammatory } \\
\text { profile, and left ventricular reverse remodeling after intracoronary delivery of } \\
\text { mesenchymal stem cells in an experimental model of pressure overload } \\
\text { hypertrophy. }\end{array}$ & 19065545 & $R$ & & ND & ND & - \\
\hline Molina* & 2009 & $\begin{array}{l}\text { Improvement in hemodynamic performance, exercise capacity, inflammatory } \\
\text { profile, and left ventricular reverse remodeling after intracoronary delivery of } \\
\text { mesenchymal stem cells in an experimental model of pressure overload } \\
\text { hypertrophy. }\end{array}$ & 19065545 & $\mathrm{R}$ & & ND & ND & - \\
\hline Donaldson & 2009 & $\begin{array}{l}\text { Estrogen Attenuates Left Ventricular and Cardiomyocyte Hypertrophy by an } \\
\text { Estrogen Receptor -Dependent Pathway That Increases Calcineurin Degradation }\end{array}$ & 19074476 & M & & - & ND & ND \\
\hline Tang* & 2008 & $\begin{array}{l}\text { Lysosomal cysteine peptidase cathepsin L protects against cardiac hypertrophy } \\
\text { through blocking AKT/GSK3beta signaling. }\end{array}$ & 19096818 & M & Ctsl $\uparrow$ & $\downarrow$ & $\downarrow$ & ND \\
\hline Tang* & 2008 & $\begin{array}{l}\text { Lysosomal cysteine peptidase cathepsin L protects against cardiac hypertrophy } \\
\text { through blocking AKT/GSK3beta signaling. }\end{array}$ & 19096818 & M & Ctsl $\uparrow$ & $\downarrow$ & $\downarrow$ & $\downarrow$ \\
\hline Nagayama & 2009 & $\begin{array}{l}\text { Sildenafil stops progressive chamber, cellular, and molecular remodeling and } \\
\text { improves calcium handling and function in hearts with pre-existing advanced } \\
\text { hypertrophy caused by pressure overload. }\end{array}$ & 19130990 & M & Pde5a $\downarrow$ & $\downarrow$ & $\downarrow$ & ND \\
\hline Lee* & 2011 & $\begin{array}{l}\text { The transcription factor Eya2 prevents pressure overload-induced adverse cardiac } \\
\text { remodeling. }\end{array}$ & 19272299 & M & Eya2 个 & $\downarrow$ & $\downarrow$ & $\downarrow$ \\
\hline Lee* & 2010 & $\begin{array}{l}\text { The transcription factor Eya2 prevents pressure overload-induced adverse cardiac } \\
\text { remodeling. }\end{array}$ & 19272299 & M & Eya2 个 & $\downarrow$ & $\downarrow$ & ND \\
\hline Lee* & 2009 & $\begin{array}{l}\text { The transcription factor Eya2 prevents pressure overload-induced adverse cardiac } \\
\text { remodeling. }\end{array}$ & 19272299 & $M$ & Eya2 个 & - & - & ND \\
\hline $\mathrm{Li}$ & 2009 & $\begin{array}{l}\text { MyD88-dependent nuclear factor-kB activation is involved in fibrinogen-induced } \\
\text { hypertrophic response of cardiomyocytes }\end{array}$ & 19342961 & $\mathrm{R}$ & & ND & ND & ND \\
\hline Levick & 2009 & Cardiac Mast Cells Mediate Left Ventricular Fibrosis in the Hypertensive Rat Heart & 19398662 & $\mathrm{R}$ & & - & $\downarrow$ & $\downarrow$ \\
\hline Cai & 2009 & $\begin{array}{l}\text { Crocetin protects against cardiac hypertrophy by blocking MEK-ERK1/2 signalling } \\
\text { pathway. }\end{array}$ & 19413885 & M & & $\downarrow$ & $\downarrow$ & $\downarrow$ \\
\hline Bian* & 2009 & $\begin{array}{l}\text { Cellular repressor of E1A-stimulated genes attenuates cardiac hypertrophy and } \\
\text { fibrosis }\end{array}$ & 19413895 & M & Creg1 $\downarrow$ & $\downarrow$ & $\downarrow$ & $\downarrow$ \\
\hline Bian* & 2009 & $\begin{array}{l}\text { Cellular repressor of E1A-stimulated genes attenuates cardiac hypertrophy and } \\
\text { fibrosis }\end{array}$ & 19413895 & M & Creg1 $\downarrow$ & $\downarrow$ & $\downarrow$ & $\downarrow$ \\
\hline Kvakan & 2009 & Regulatory T cells ameliorate angiotensin II-induced cardiac damage. & 19470887 & M & & $\downarrow$ & $\downarrow$ & $\downarrow$ \\
\hline Hikoso & 2009 & The IkB Kinase b/Nuclear Factor kB Signaling Pathway Protects the Heart From & 19478205 & M & $I k b k b \downarrow$ & $\uparrow$ & $\uparrow$ & ND \\
\hline
\end{tabular}


Nakamura*

Panek

Moens

Honsho*

Honsho*

Levick

Kimura*

Shen

Haudek*

Haudek*

Haudek*

\section{Cai*}

Cai*

$\mathrm{Ai}$

Huang

Mohammed

Heineke

Sumitomo-

Ueda

Usher

Kuroda
Dismutase Expression

Liver $\mathrm{X}$ receptors are negative regulators of cardiac hypertrophy via suppressing NF-kappaB signalling.

Cardiac 12/15 lipoxygenase-induced inflammation is involved in heart failure. Critical role of apoptosis signal-regulating kinase 1 in aldosterone/salt-induced cardiac inflammation and fibrosis.

Critical role of apoptosis signal-regulating kinase 1 in aldosterone/salt-induced cardiac inflammation and fibrosis.

Nrf2 protects against maladaptive cardiac responses to hemodynamic stress. NF-kappaB activation is required for adaptive cardiac hypertrophy. Deficiency of nectin-2 leads to cardiac fibrosis and dysfunction under chronic pressure overload.

Exaggerated blood pressure variability superimposed on hypertension aggravates cardiac remodeling in rats via angiotensin II system-mediated chronic inflammation.

Connective tissue growth factor overexpression in cardiomyocytes promotes cardiac hypertrophy and protection against pressure overload.

Adverse ventricular remodeling and exacerbated NOS uncoupling from pressureoverload in mice lacking the beta3-adrenoreceptor.

Myocardial fibrosis and apoptosis, but not inflammation, are present in long-term experimental diabetes.

Pressure-mediated hypertrophy and mechanical stretch induces IL-1 release and subsequent IGF-1 generation to maintain compensative hypertrophy by affecting Akt and JNK pathways.

Pressure-mediated hypertrophy and mechanical stretch induces IL-1 release and subsequent IGF-1 generation to maintain compensative hypertrophy by affecting Akt and JNK pathways.

Antioxidant amelioration of dilated cardiomyopathy caused by conditional deletion of NEMO/IKKgamma in cardiomyocytes

Evidence for the importance of adiponectin in the cardioprotective effects of pioglitazone.

Evidence for the importance of adiponectin in the cardioprotective effects of pioglitazone.

Sympathetic nervous system modulation of inflammation and remodeling in the hypertensive heart.

Targeted deletion of the extracellular signal-regulated protein kinase 5 attenuates hypertrophic response and promotes pressure overload-induced apoptosis in the heart.

Targeted deletion of the extracellular signal-regulated protein kinase 5 attenuate hypertrophic response and promotes pressure overload-induced apoptosis in the heart.

Tetrandrine blocks cardiac hypertrophy by disrupting reactive oxygen speciesdependent ERK1/2 signalling.

Breviscapine protects against cardiac hypertrophy through blocking PKC-alphadependent signaling.

C-reactive protein promotes cardiac fibrosis and inflammation in angiotensin IIinduced hypertensive cardiac disease.

Allicin protects against cardiac hypertrophy and fibrosis via attenuating reactive oxygen species-dependent signaling pathways

Adiponectin deficiency exacerbates cardiac dysfunction following pressure overload through disruption of an AMPK-dependent angiogenic response.

Adenylyl cyclase 6 deletion reduces left ventricular hypertrophy, dilation, dysfunction, and fibrosis in pressure-overloaded female mice. Krüppel-like factor 4 regulates pressure-induced cardiac hypertrophy.

Chronic urotensin II receptor antagonist treatment does not alter hypertrophy or fibrosis in a rat model of pressure-overload hypertrophy.

Monocytic fibroblast precursors mediate fibrosis in angiotensin-II-induced cardiac hypertrophy.

Monocytic fibroblast precursors mediate fibrosis in angiotensin-II-induced cardiac hypertrophy.

2010 Monocytic fibroblast precursors mediate fibrosis in angiotensin-II-induced cardiac hypertrophy.

2010 Intracellular or extracellular heat shock protein 70 differentially regulates cardiac remodelling in pressure overload mice.

Intracellular or extracellular heat shock protein 70 differentially regulates cardiac remodelling in pressure overload mice.

Silibinin attenuates cardiac hypertrophy and fibrosis through blocking EGFRdependent signaling.

2010 Tumor suppressor A20 protects against cardiac hypertrophy and fibrosis by blocking transforming growth factor-beta-activated kinase 1-dependent signaling. Mineralocorticoid accelerates transition to heart failure with preserved ejection fraction via "nongenomic effects".

CIB1 is a regulator of pathological cardiac hypertrophy.

Heparin cofactor II protects against angiotensin II-induced cardiac remodeling via attenuation of oxidative stress in mice.

Myeloid mineralocorticoid receptor controls macrophage polarization and cardiovascular hypertrophy and remodeling in mice.

2010 NADPH oxidase 4 (Nox4) is a major source of oxidative stress in the failing heart.

\begin{tabular}{|c|c|c|c|c|}
\hline 19487338 & $M$ & $N r 1 h 3 \downarrow$ & $\uparrow$ & ND \\
\hline 19546247 & $M$ & Alox15 $\downarrow$ & $\downarrow$ & ND \\
\hline 19581502 & $M$ & Map $3 k 5 \downarrow$ & - & $\downarrow$ \\
\hline 19581502 & $M$ & Map3k5 $\downarrow$ & - & $\downarrow$ \\
\hline 19592468 & $M$ & $N r f 2 \downarrow$ & $\uparrow$ & $\uparrow$ \\
\hline 19620128 & $M$ & $N f k b i a \downarrow$ & - & - \\
\hline 19667252 & $M$ & Pvrl2 $\downarrow$ & - & $\uparrow$ \\
\hline 19704105 & $\mathrm{R}$ & & $\uparrow$ & $\uparrow$ \\
\hline 19707545 & $M$ & $\operatorname{Ctgf} \uparrow$ & ND & - \\
\hline 19766235 & $M$ & $A d r a 2 b \downarrow$ & $\uparrow$ & $\uparrow$ \\
\hline 19820199 & $\mathrm{R}$ & & $\uparrow$ & - \\
\hline 19834007 & $M$ & $\| 1 b \downarrow$ & $\downarrow$ & ND \\
\hline 19834007 & $M$ & $\| 1 b \downarrow$ & $\downarrow$ & $\uparrow$ \\
\hline 19850942 & $M$ & Ikbkg $\downarrow$ & $\uparrow$ & $\uparrow$ \\
\hline 19933924 & $M$ & Adipoq $\downarrow$ & $\uparrow$ & - \\
\hline 19933924 & $M$ & & $\downarrow$ & $\downarrow$ \\
\hline 20048196 & $\mathrm{R}$ & & $\downarrow$ & $\downarrow$ \\
\hline 20075332 & $M$ & Mapk7 $\downarrow$ & $\downarrow$ & $\downarrow$ \\
\hline 20075332 & $M$ & Mapk7 $\downarrow$ & $\downarrow$ & $\downarrow$ \\
\hline 20105174 & $M$ & & $\downarrow$ & $\downarrow$ \\
\hline 20127712 & $M$ & & $\downarrow$ & $\downarrow$ \\
\hline 20157054 & $M$ & Crp $\uparrow$ & ND & $\uparrow$ \\
\hline 20185286 & $M$ & & $\downarrow$ & $\downarrow$ \\
\hline 20206634 & $M$ & Adipoq $\downarrow$ & $\uparrow$ & $\uparrow$ \\
\hline 20359598 & $M$ & Adcy $6 \downarrow$ & $\downarrow$ & $\downarrow$ \\
\hline 20433848 & $M$ & $K l f 4 \downarrow$ & $\uparrow$ & $\uparrow$ \\
\hline 20452383 & $\mathrm{R}$ & $U t s 2 b \downarrow$ & - & - \\
\hline 20488188 & $M$ & $C c / 2 \downarrow$ & - & $\downarrow$ \\
\hline 20488188 & $M$ & $C c 12 \downarrow$ & - & $\downarrow$ \\
\hline 20488188 & $M$ & $C c / 2 \downarrow$ & - & $\downarrow$ \\
\hline 20542874 & $M$ & $H s p a 1 b \downarrow$ & $\uparrow$ & $\downarrow$ \\
\hline 20542874 & $M$ & Hspa1b $\downarrow$ & $\downarrow$ & $\downarrow$ \\
\hline 20564207 & $M$ & & $\downarrow$ & $\downarrow$ \\
\hline 20585109 & $M$ & Tnfaip3 $\uparrow$ & $\downarrow$ & $\downarrow$ \\
\hline 20625113 & $M$ & & $\uparrow$ & $\uparrow$ \\
\hline 20639889 & $M$ & $C i b 1 \downarrow$ & $\downarrow$ & $\downarrow$ \\
\hline 20660821 & $M$ & Serpind $1 \downarrow$ & - & $\uparrow$ \\
\hline 20697155 & $M$ & $N r 3 c 2 \downarrow$ & $\downarrow$ & $\downarrow$ \\
\hline 20713697 & $M$ & Nox4 $\downarrow$ & $\downarrow$ & $\downarrow$ \\
\hline
\end{tabular}




\begin{tabular}{|c|c|c|c|c|c|c|c|c|}
\hline Serpi & 2010 & $\begin{array}{l}\text { Vascular endothelial growth factor-B gene transfer prevents angiotensin II-induced } \\
\text { diastolic dysfunction via proliferation and capillary dilatation in rats. }\end{array}$ & 20733007 & $\mathrm{R}$ & Vegfb $\uparrow$ & ND & $\downarrow$ & ND \\
\hline Song & 2010 & $\begin{array}{l}\text { mTOR attenuates the inflammatory response in cardiomyocytes and prevents } \\
\text { cardiac dysfunction in pathological hypertrophy. }\end{array}$ & 20861467 & $M$ & $m T O R \downarrow$ & $\downarrow$ & $\downarrow$ & $\downarrow$ \\
\hline Del Re & 2010 & $\begin{array}{l}\text { Proapoptotic Rassf1A/Mst1 signaling in cardiac fibroblasts is protective against } \\
\text { pressure overload in mice. }\end{array}$ & 20890045 & $M$ & Rassf1 $\downarrow$ & $\uparrow$ & $\uparrow$ & ND \\
\hline Li* & 2010 & $\begin{array}{l}\text { Cellular FLICE-inhibitory protein protects against cardiac remodeling induced by } \\
\text { angiotensin II in mice. }\end{array}$ & 20975036 & $M$ & Cflar $\downarrow$ & $\uparrow$ & $\uparrow$ & ND \\
\hline $\mathrm{Li}^{*}$ & 2010 & $\begin{array}{l}\text { Cellular FLICE-inhibitory protein protects against cardiac remodeling induced by } \\
\text { angiotensin II in mice. }\end{array}$ & 20975036 & $M$ & Cflar $\uparrow$ & $\downarrow$ & $\downarrow$ & ND \\
\hline Patrick* & 2010 & $\begin{array}{l}\text { Stress-dependent cardiac remodeling occurs in the absence of microRNA- } 21 \text { in } \\
\text { mice. }\end{array}$ & 20978354 & $M$ & Mir21 $\downarrow$ & - & - & ND \\
\hline Patrick* & 2011 & $\begin{array}{l}\text { Stress-dependent cardiac remodeling occurs in the absence of microRNA- } 21 \text { in } \\
\text { mice. }\end{array}$ & 20978355 & $M$ & Mir21 $\downarrow$ & - & - & ND \\
\hline Harding & 2011 & $\begin{array}{l}\text { Lack of microsomal prostaglandin E synthase-1 reduces cardiac function following } \\
\text { angiotensin II infusion. }\end{array}$ & 21193590 & $M$ & Ptges $\downarrow$ & - & - & ND \\
\hline Sopko & 2010 & Bone marrow support of the heart in pressure overload is lost with aging. & 21203577 & M & & $\uparrow$ & ND & - \\
\hline Nagai* & 2011 & $\begin{array}{l}\text { C-reactive protein overexpression exacerbates pressure overload-induced cardiac } \\
\text { remodeling through enhanced inflammatory response. }\end{array}$ & 21220701 & M & Crp 个 & $\uparrow$ & - & $\uparrow$ \\
\hline Nagai* & 2011 & $\begin{array}{l}\text { C-reactive protein overexpression exacerbates pressure overload-induced cardiac } \\
\text { remodeling through enhanced inflammatory response. }\end{array}$ & 21220701 & M & Crp 个 & - & $\uparrow$ & $\uparrow$ \\
\hline Skoumal & 2011 & $\begin{array}{l}\text { Parthenolide inhibits STAT3 signaling and attenuates angiotensin II-induced left } \\
\text { ventricular hypertrophy via modulation of fibroblast activity. }\end{array}$ & 21223972 & $\mathrm{R}$ & Stat3 $\downarrow$ & $\downarrow$ & $\downarrow$ & $\downarrow$ \\
\hline Givvimani* & 2011 & $\begin{array}{l}\text { Hydrogen sulfide mitigates transition from compensatory hypertrophy to heart } \\
\text { failure. }\end{array}$ & 21233344 & M & & ND & $\downarrow$ & ND \\
\hline Givvimani* & 2011 & $\begin{array}{l}\text { Hydrogen sulfide mitigates transition from compensatory hypertrophy to heart } \\
\text { failure. }\end{array}$ & 21233344 & $M$ & & ND & $\downarrow$ & ND \\
\hline Shimano & 2011 & $\begin{array}{l}\text { Cardiac myocyte-specific ablation of follistatin-like } 3 \text { attenuates stress-induced } \\
\text { myocardial hypertrophy }\end{array}$ & 21245136 & M & Fst/3 $\downarrow$ & $\downarrow$ & $\downarrow$ & ND \\
\hline Chen & 2011 & $\begin{array}{l}\text { Omega-3 fatty acids prevent pressure overload-induced cardiac fibrosis through } \\
\text { activation of cyclic GMP/protein kinase G signaling in cardiac fibroblasts. }\end{array}$ & 21282499 & $M$ & & $\downarrow$ & $\downarrow$ & $\downarrow$ \\
\hline Liu & 2011 & $\begin{array}{l}\text { Deprivation of MKK7 in cardiomyocytes provokes heart failure in mice when } \\
\text { exposed to pressure overload. }\end{array}$ & 21284947 & $M$ & Map2k7 $\downarrow$ & $\uparrow$ & ND & $\uparrow$ \\
\hline Lother* & 2011 & $\begin{array}{l}\text { Ablation of mineralocorticoid receptors in myocytes but not in fibroblasts } \\
\text { preserves cardiac function. }\end{array}$ & 21321305 & $M$ & $N r 3 c 2 \downarrow$ & $\uparrow$ & - & - \\
\hline Lother* & 2011 & $\begin{array}{l}\text { Ablation of mineralocorticoid receptors in myocytes but not in fibroblasts } \\
\text { preserves cardiac function. }\end{array}$ & 21321305 & M & $N r 3 c 2 \downarrow$ & - & - & - \\
\hline$Q i$ & 2011 & $\begin{array}{l}\text { Angiotensin II infusion-induced inflammation, monocytic fibroblast precursor } \\
\text { infiltration, and cardiac fibrosis are pressure dependent. }\end{array}$ & 21373977 & $M$ & & $\downarrow$ & $\downarrow$ & $\downarrow$ \\
\hline Zhang & 2011 & HSP75 protects against cardiac hypertrophy and fibrosis. & 21381076 & M & Trap1 个 & $\downarrow$ & $\downarrow$ & ND \\
\hline Aceros* & 2011 & $\begin{array}{l}\text { Moxonidine improves cardiac structure and performance in SHR through inhibition } \\
\text { of cytokines, p38 MAPK and Akt }\end{array}$ & 21426316 & $\mathrm{R}$ & & $\downarrow$ & $\downarrow$ & $\downarrow$ \\
\hline Aceros* & 2011 & $\begin{array}{l}\text { Moxonidine improves cardiac structure and performance in SHR through inhibition } \\
\text { of cytokines, p38 MAPK and Akt }\end{array}$ & 21426316 & $\mathrm{R}$ & & $\downarrow$ & ND & $\downarrow$ \\
\hline Ren & 2011 & $\begin{array}{l}\text { Proinflammatory protein CARD9 is essential for infiltration of monocytic fibroblast } \\
\text { precursors and cardiac fibrosis caused by Angiotensin II infusion. }\end{array}$ & 21436792 & M & Card9 $\downarrow$ & - & $\downarrow$ & $\downarrow$ \\
\hline Gao & 2011 & $\begin{array}{l}\text { High salt intake accelerated cardiac remodeling in spontaneously hypertensive rats: } \\
\text { time window of left ventricular functional transition and its relation to salt-loading } \\
\text { doses. }\end{array}$ & 21529316 & $\mathrm{R}$ & & $\uparrow$ & $\uparrow$ & ND \\
\hline Liu & 2011 & $\begin{array}{l}\text { Lentivirus mediated IL-17R blockade improves diastolic cardiac function in } \\
\text { spontaneously hypertensive rats. }\end{array}$ & 21530504 & $\mathrm{R}$ & II17ra $\downarrow$ & $\downarrow$ & $\downarrow$ & ND \\
\hline Waehre & 2011 & $\begin{array}{l}\text { Lack of chemokine signaling through CXCR5 causes increased mortality, ventricular } \\
\text { dilatation and deranged matrix during cardiac pressure overload. }\end{array}$ & 21533157 & $M$ & $C x c r 5 \downarrow$ & $\uparrow$ & $\uparrow$ & - \\
\hline Zhu & 2011 & $\begin{array}{l}\text { The TIR/BB-loop mimetic AS-1 prevents cardiac hypertrophy by inhibiting IL-1R- } \\
\text { mediated MyD88-dependent signaling }\end{array}$ & 21533832 & $M$ & & $\downarrow$ & $\downarrow$ & $\downarrow$ \\
\hline $\mathrm{Xu}$ & 2011 & $\begin{array}{l}\text { CCR2 mediates the uptake of bone marrow-derived fibroblast precursors in } \\
\text { angiotensin II-induced cardiac fibrosis. }\end{array}$ & 21572015 & $M$ & $C c r 2 \downarrow$ & - & $\downarrow$ & ND \\
\hline Peng & 2011 & Angiotensin II-induced dilated cardiomyopathy in Balb/c but not C57BL/6J mice. & 21602297 & M & & - & $\uparrow$ & ND \\
\hline Ehrentraut & 2011 & The toll-like receptor 4-antagonist eritoran reduces murine cardiac hypertrophy & 21613426 & M & Tlr4 $\downarrow$ & $\downarrow$ & ND & $\downarrow$ \\
\hline Usui* & 2011 & $\begin{array}{l}\text { Endogenous muscle atrophy F-box mediates pressure overload-induced cardiac } \\
\text { hypertrophy through regulation of nuclear factor-kappaB. }\end{array}$ & 21617130 & M & Fbxo32 $\downarrow$ & $\downarrow$ & $\downarrow$ & $\downarrow$ \\
\hline Usui* & 2011 & $\begin{array}{l}\text { Endogenous muscle atrophy F-box mediates pressure overload-induced cardiac } \\
\text { hypertrophy through regulation of nuclear factor-kappaB. }\end{array}$ & 21617130 & M & $F b x o 32 \downarrow$ & $\downarrow$ & ND & ND \\
\hline Yan* & 2011 & $\begin{array}{l}\text { Cardiac-specific mindin overexpression attenuates cardiac hypertrophy via blocking } \\
\text { AKT/GSK3 } \beta \text { and TGF- } \beta 1 \text {-Smad signalling. }\end{array}$ & 21632881 & $M$ & Spon2 $\uparrow$ & $\downarrow$ & $\downarrow$ & ND \\
\hline Yan* & 2011 & $\begin{array}{l}\text { Cardiac-specific mindin overexpression attenuates cardiac hypertrophy via blocking } \\
\text { AKT/GSK3 } \beta \text { and TGF- } \beta 1 \text {-Smad signalling. }\end{array}$ & 21632881 & $M$ & Spon2 $\uparrow$ & $\downarrow$ & $\downarrow$ & ND \\
\hline $\mathrm{Xu}$ & 2011 & $\begin{array}{l}\text { IKB } \beta \text { attenuates angiotensin II-induced cardiovascular inflammation and fibrosis in } \\
\text { mice. }\end{array}$ & 21646597 & $M$ & $N f k b i a \downarrow$ & ND & $\downarrow$ & $\downarrow$ \\
\hline Mandl & 2011 & $\begin{array}{l}\text { Puma deletion delays cardiac dysfunction in murine heart failure models through } \\
\text { attenuation of apoptosis. }\end{array}$ & 21670227 & $M$ & $B b c 3 \downarrow$ & - & $\downarrow$ & ND \\
\hline Garlie* & 2011 & $\begin{array}{l}\text { Tumor necrosis factor receptor } 2 \text { signaling limits } \beta \text {-adrenergic receptor-mediated } \\
\text { cardiac hypertrophy in vivo. }\end{array}$ & 21691899 & $M$ & Tnfrsfla $\downarrow$ & - & - & $\downarrow$ \\
\hline Garlie* & 2011 & $\begin{array}{l}\text { Tumor necrosis factor receptor } 2 \text { signaling limits } \beta \text {-adrenergic receptor-mediated } \\
\text { cardiac hypertrophy in vivo. }\end{array}$ & 21691899 & M & Tnfrsf1a $\downarrow$ & $\downarrow$ & ND & ND \\
\hline Garlie* & 2011 & $\begin{array}{l}\text { Tumor necrosis factor receptor } 2 \text { signaling limits } \beta \text {-adrenergic receptor-mediated } \\
\text { cardiac hypertrophy in vivo. }\end{array}$ & 21691899 & $M$ & Tnfrsf $1 b \downarrow$ & $\uparrow$ & - & - \\
\hline Garlie* & 2011 & Tumor necrosis factor receptor 2 signaling limits $\beta$-adrenergic receptor-mediated & 21691899 & M & Tnfrsf $1 b \downarrow$ & - & ND & ND \\
\hline
\end{tabular}


Chan

2011

Steinbusch*

2011

Steinbusch*

2011

Iyer

2011

Castoldi

2011

Georgescu

Kanellakis

Buckley

Shu

Carnevale

2011

2011

2011

Xia

Rosenblatt

Velin

Zou

Zhou

Finckenberg

Zou*

Zou*

Zou*

Zou*

Kazakov

$\mathrm{He}$

Gaspar-Pereira

Martín-

Fernández

Frolova

Adiarto

Bian*

Bian*

Wei

Shang*

2012

2012

2012

Shang*

2012

2012

Kanellakis

Wu

Park

Oka

Yang

Wang

2012

Han

$\mathrm{Ma}$

Valente

Xiao

2012 cardiac hypertrophy in vivo.

Chronic b-adrenoceptor Antagonist Treatment Controls Cardiovascular Remodeling in Heart Failure in the Aging Spontaneously Hypertensive Rat

Absence of fatty acid transporter CD36 protects against Western-type diet-related cardiac dysfunction following pressure overload in mice.

Absence of fatty acid transporter CD36 protects against Western-type diet-related cardiac dysfunction following pressure overload in mice.

Inhibition of inflammation and fibrosis by a complement C5a receptor antagonist in DOCA-salt hypertensive rats.

MiR-133a Regulates Collagen1A1: Potential Role of miR-133a in Myocardial Fibrosis in Angiotensin II-Dependent Hypertension

Decreased metalloprotease 9 induction, cardiac fibrosis, and higher autophagy after pressure overload in mice lacking the transcriptional regulator $\mathrm{p} 8$.

$\mathrm{CD}^{+} \mathrm{CD} 25^{+} \mathrm{Foxp}^{+}$regulatory T cells suppress cardiac fibrosis in the hypertensive heart.

Mice lacking functional TRPV1 are protected from pressure overload cardiac hypertrophy.

Gastrodin protects against cardiac hypertrophy and fibrosis.

Placental Growth Factor Regulates Cardiac Inflammation Through the Tissue Inhibitor of Metalloproteinases-3/Tumor Necrosis Factor a Converting Enzyme Axis: Crucial Role for Adaptive Cardiac Remodeling During Cardiac Pressure Overload Endogenous thrombospondin 1 protects the pressure-overloaded myocardium by modulating fibroblast phenotype and matrix metabolism.

Cardiac dysfunction and impaired compensatory response to pressure overload in mice deficient in stem cell antigen-1.

Ryanodine receptor type 2 is required for the development of pressure overloadinduced cardiac hypertrophy.

Activating transcription factor 3 deficiency promotes cardiac hypertrophy, dysfunction, and fibrosis induced by pressure overload.

Caloric restriction ameliorates angiotensin II-induced mitochondrial remodeling and cardiac hypertrophy.

Qiliqiangxin inhibits the development of cardiac hypertrophy, remodeling, and

dysfunction during 4 weeks of pressure overload in mice.

Qiliqiangxin inhibits the development of cardiac hypertrophy, remodeling, and

dysfunction during 4 weeks of pressure overload in mice.

Qiliqiangxin inhibits the development of cardiac hypertrophy, remodeling, and dysfunction during 4 weeks of pressure overload in mice.

Qiliqiangxin inhibits the development of cardiac hypertrophy, remodeling, and

dysfunction during 4 weeks of pressure overload in mice.

Endothelial nitric oxide synthase of the bone marrow regulates myocardial

hypertrophy, fibrosis, and angiogenesis.

In vivo and in vitro protective effects of pentamethylquercetin on cardiac hypertrophy.

The NF-kB subunit c-Rel stimulates cardiac hypertrophy and fibrosis.

Spironolactone prevents alterations associated with cardiac hypertrophy produced

by isoproterenol in rats: involvement of serum- and glucocorticoid-regulated kinase

type 1.

Thrombospondin- 4 regulates fibrosis and remodeling of the myocardium in response to pressure overload.

ET-1 from endothelial cells is required for complete angiotensin II-induced cardiac fibrosis and hypertrophy.

Disruption of mindin exacerbates cardiac hypertrophy and fibrosis.

Disruption of mindin exacerbates cardiac hypertrophy and fibrosis.

Endothelial expression of hypoxia-inducible factor 1 protects the murine heart and aorta from pressure overload by suppression of TGF- $\beta$ signaling.

Involvement of reactive oxygen species and JNK in increased expression of MCP-1 and infiltration of inflammatory cells in pressure-overloaded rat hearts.

Involvement of reactive oxygen species and JNK in increased expression of MCP-1 and infiltration of inflammatory cells in pressure-overloaded rat hearts.

A pro-fibrotic role for interleukin- 4 in cardiac pressure overload.

Hexokinase II knockdown results in exaggerated cardiac hypertrophy via increased ROS production.

The chemical chaperone 4-phenylbutyric acid attenuates pressure-overload cardiac hypertrophy by alleviating endoplasmic reticulum stress.

Mitochondrial DNA that escapes from autophagy causes inflammation and heart failure

Serum-glucocorticoid regulated kinase 1 regulates alternatively activated macrophage polarization contributing to angiotensin II-induced inflammation and cardiac fibrosis.

Promyelocytic leukemia zinc finger protein activates GATA4 transcription and mediates cardiac hypertrophic signaling from angiotensin II receptor 2.

2012 Reciprocal interaction between macrophages and T cells stimulates IFN- $\gamma$ and MCP1 production in Ang II-induced cardiac inflammation and fibrosis.

Macrophage-stimulated cardiac fibroblast production of IL- 6 is essential for TGF

$\beta /$ Smad activation and cardiac fibrosis induced by angiotensin II.

CIKS (Act1 or TRAF3IP2) mediates Angiotensin-II-induced Interleukin-18 expression,

and Nox2-dependent cardiomyocyte hypertrophy.

Augmented cardiac hypertrophy in response to pressure overload in mice lacking
21709583

21712535

21712535

21753735

21769867

21775709

21785365

21814047

21833534

21900081

21947471

21957128

21986507

22053207

22068868

22075750

22075750

22075750

22075750

22106415

22183794

22210479

22327331

22362893

22365964

22367478

22367478

22403061

22470108

22470108

22492684

22517678

22525677

22535248

22556335

22558183

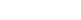

22567105

22574112

22575763

22606234
$\mathrm{R} \quad \downarrow \quad \downarrow \quad N D$

M Cd36 $\downarrow \quad \uparrow \quad N D \quad \uparrow$

Cd36 $\downarrow \quad-\quad N D \quad \downarrow$

$\operatorname{C5ar} 1 \downarrow \quad \downarrow \quad \downarrow \quad \downarrow$

$\downarrow \quad \downarrow \quad N D$

$p 8 \downarrow \quad-\quad \downarrow \quad \downarrow$

$\downarrow \quad \downarrow \quad \downarrow$

$\operatorname{Trpv} 1 \downarrow \quad \downarrow \quad \downarrow \quad \downarrow$

$\operatorname{Pgf} \downarrow \quad \downarrow \quad \downarrow \quad$ ND

Thbs1 $\downarrow \quad \uparrow$

$S c a 1 \downarrow \quad \uparrow \quad$ ND ND

$R y r 2 \downarrow \quad \downarrow \quad \downarrow \quad N D$

Atf $\downarrow \downarrow \quad \uparrow \quad \uparrow \quad N D$

$\downarrow \quad \downarrow \quad N D$

$\downarrow \quad \downarrow \quad \downarrow$

$\downarrow \quad \downarrow \quad \downarrow$

$\downarrow \quad \downarrow \quad \downarrow$

$\downarrow \quad \downarrow \quad \downarrow$

Nos3 $\downarrow \quad \downarrow \quad \uparrow \quad N D$

$\operatorname{Rel} \downarrow$

$\downarrow \quad \downarrow \quad \downarrow$

$\downarrow \quad \downarrow \quad$ ND

R $\downarrow \downarrow \downarrow \downarrow$

Thbs4 $\quad \uparrow \quad \uparrow \quad \downarrow$

$E d n 1 \downarrow \quad \downarrow \quad \downarrow \quad N D$

Spon2 $\downarrow \quad \uparrow \quad \uparrow \quad$ ND

Spon2 $\downarrow \quad \uparrow \quad \uparrow \quad$ ND

Hif1a $\downarrow-\quad$ ND

$\mathrm{R} \quad \downarrow \quad \downarrow \quad$ ND

$R$

$\mathrm{M} \quad 1 / 4 \downarrow$

$H k 2 \downarrow$

$\downarrow \quad \downarrow \quad N D$

$\downarrow \quad \downarrow \quad N D$

$\uparrow \uparrow$ ND

$\downarrow \quad \downarrow \quad N D$

Dnase2a $\uparrow \uparrow \uparrow \uparrow$

$S g k 1 \downarrow \quad \downarrow \quad \downarrow \quad \downarrow$

Zbtb16 $\downarrow \quad \downarrow \quad \downarrow \quad N D$

Ifng $\downarrow \quad-\quad \downarrow \quad \downarrow$

$116 \downarrow \quad-\quad \downarrow \quad \downarrow$

M Traf3ip $2 \downarrow \quad \downarrow \quad \downarrow \quad \downarrow$

M Adgrl4 $\downarrow \quad \uparrow \quad \uparrow \quad$ ND 
Takatsu*

Meems

Zhou*

Nagai*

Nagai*

Cittadini

Velten*

Velten*

Velten*

Velten*

Velten*

Velten*

Sekinishi

Tanaka

Markó
ELTD1.

Effects of the absence of procollagen C-endopeptidase enhancer-2 on myocardial collagen accumulation in chronic pressure overload.

CXCR4 gene transfer prevents pressure overload induced heart failure CXCR4 gene transfer prevents pressure overload induced heart failure Interleukin-10 treatment attenuates pressure overload-induced hypertrophic remodeling and improves heart function via signal transducers and activators of transcription 3-dependent inhibition of nuclear factor-kB.

Interleukin-10 treatment attenuates pressure overload-induced hypertrophic remodeling and improves heart function via signal transducers and activators of transcription 3-dependent inhibition of nuclear factor-kB.

Interleukin-10 treatment attenuates pressure overload-induced hypertrophic remodeling and improves heart function via signal transducers and activators of transcription 3-dependent inhibition of nuclear factor-kB.

Carboxyl terminus of heat shock protein 70 -interacting protein inhibits angiotensin II-induced cardiac remodeling.

Interferon- $\gamma$ ablation exacerbates myocardial hypertrophy in diastolic heart failure. Cardiac-specific deletion of acetyl CoA carboxylase 2 prevents metabolic remodeling during pressure-overload hypertrophy.

Cardioprotection of controlled and cardiac-specific over-expression of A(2A)adenosine receptor in the pressure overload.

Comparison of the effects of cilnidipine and amlodipine on cardiac remodeling and diastolic dysfunction in Dahl salt-sensitive rats.

Comparison of the effects of cilnidipine and amlodipine on cardiac remodeling and diastolic dysfunction in Dahl salt-sensitive rats.

The vitamin $\mathrm{D}$ receptor activator paricalcitol prevents fibrosis and diastolic dysfunction in a murine model of pressure overload.

Depletion of endogenous kallistatin exacerbates renal and cardiovascular oxidative stress, inflammation, and organ remodeling.

Pressure overload-induced cardiac remodeling and dysfunction in the absence of interleukin 6 in mice

Pressure overload-induced cardiac remodeling and dysfunction in the absence of interleukin 6 in mice.

Chronic administration of hexarelin attenuates cardiac fibrosis in the spontaneously hypertensive rat.

Chronic C-Type Natriuretic Peptide Infusion Attenuates Angiotensin II-Induced Myocardial Superoxide Production and Cardiac Remodeling.

Stem cell antigen 1 protects against cardiac hypertrophy and fibrosis after pressure overload.

Stem cell antigen 1 protects against cardiac hypertrophy and fibrosis after pressure overload.

Determining the absolute requirement of $\mathrm{G}$ protein-coupled receptor kinase 5 for pathological cardiac hypertrophy: short communication.

Eicosapentaenoic acid suppresses adverse effects of C-reactive protein overexpression on pressure overload-induced cardiac remodeling. Eicosapentaenoic acid suppresses adverse effects of $\mathrm{C}$-reactive protein overexpression on pressure overload-induced cardiac remodeling. SOCS1 gene transfer accelerates the transition to heart failure through the inhibition of the gp130/JAK/STAT pathway

Targeted disruption of Hspa4 gene leads to cardiac hypertrophy and fibrosis. Coronary artery remodeling in a model of left ventricular pressure overload is influenced by platelets and inflammatory cells.

Priming with synthetic oligonucleotides attenuates pressure overload-induced inflammation and cardiac hypertrophy in mice.

Priming with synthetic oligonucleotides attenuates pressure overload-induced inflammation and cardiac hypertrophy in mice.

Priming with synthetic oligonucleotides attenuates pressure overload-induced inflammation and cardiac hypertrophy in mice.

Priming with synthetic oligonucleotides attenuates pressure overload-induced inflammation and cardiac hypertrophy in mice.

Priming with synthetic oligonucleotides attenuates pressure overload-induced inflammation and cardiac hypertrophy in mice.

Priming with synthetic oligonucleotides attenuates pressure overload-induced inflammation and cardiac hypertrophy in mice.

Periodontal pathogen Aggregatibacter actinomycetemcomitans deteriorates pressure overload-induced myocardial hypertrophy in mice.

Inhibition of IKB phosphorylation prevents load-induced cardiac dysfunction in mice.

Interferon- $\gamma$ signaling inhibition ameliorates angiotensin II-induced cardiac damage.

Selective PDE5A inhibition with sildenafil rescues left ventricular dysfunction, inflammatory immune response and cardiac remodeling in angiotensin II-induced heart failure in vivo.

Chronic depletion of glutathione exacerbates ventricular remodelling and dysfunction in the pressure-overloaded heart.

Proteasome inhibition attenuates heart failure during the late stages of pressure overload through alterations in collagen expression

Macrophage migration inhibitory factor antagonizes pressure overload-induced

\begin{tabular}{|c|c|c|c|c|}
\hline 22610170 & $M$ & Pcolce2 $\downarrow$ & $\downarrow$ & $\downarrow$ \\
\hline 22668785 & $M$ & Cxcr4 个 & $\downarrow$ & ND \\
\hline 22668785 & M & Cxcr4 个 & $\downarrow$ & ND \\
\hline 22705886 & $M$ & $1 / 10 \downarrow$ & $\uparrow$ & $\uparrow$ \\
\hline 22705886 & $M$ & $\| 10 \downarrow$ & $\uparrow$ & $\uparrow$ \\
\hline 22705886 & $M$ & $1110 \uparrow$ & $\downarrow$ & $\downarrow$ \\
\hline 22717542 & $M$ & Stub1 个 & - & $\downarrow$ \\
\hline 22730392 & $M$ & Ifng $\downarrow$ & $\uparrow$ & - \\
\hline 22730442 & $M$ & $A c a c b \downarrow$ & $\downarrow$ & $\downarrow$ \\
\hline 22792196 & M & Adora $2 a \uparrow$ & $\downarrow$ & $\downarrow$ \\
\hline 22796710 & $\mathrm{R}$ & & $\downarrow$ & $\downarrow$ \\
\hline 22796710 & $\mathrm{R}$ & & $\downarrow$ & $\downarrow$ \\
\hline 22800987 & $M$ & $V d r \uparrow$ & - & $\downarrow$ \\
\hline 22811485 & $\mathrm{R}$ & & - & $\uparrow$ \\
\hline 22825686 & $M$ & $116 \downarrow$ & - & - \\
\hline 22825686 & M & $116 \downarrow$ & - & - \\
\hline 22842067 & $\mathrm{R}$ & & $\downarrow$ & $\downarrow$ \\
\hline 22848833 & $M$ & & $\downarrow$ & $\downarrow$ \\
\hline 22851736 & M & Sca1 $\downarrow$ & $\uparrow$ & $\uparrow$ \\
\hline 22851736 & $M$ & Sca1 $\uparrow$ & $\downarrow$ & $\downarrow$ \\
\hline 22859683 & $M$ & Grk5 $\downarrow$ & $\downarrow$ & $\downarrow$ \\
\hline 22875408 & $M$ & Crp $\uparrow$ & $\uparrow$ & $\uparrow$ \\
\hline 22875408 & $M$ & Crp $\uparrow$ & - & $\uparrow$ \\
\hline 22875468 & $\mathrm{R}$ & Socs1 $\uparrow$ & - & $\uparrow$ \\
\hline 22884543 & $M$ & Hspa4 $\downarrow$ & $\uparrow$ & $\uparrow$ \\
\hline 22916095 & M & $\operatorname{Rag} 1 \downarrow$ & - & - \\
\hline 22977006 & $M$ & & - & $\downarrow$ \\
\hline 22977006 & $M$ & & $\downarrow$ & $\downarrow$ \\
\hline 22977006 & $M$ & & $\downarrow$ & $\downarrow$ \\
\hline 22977006 & $M$ & & $\downarrow$ & - \\
\hline 22977006 & $M$ & & - & ND \\
\hline 22977006 & $M$ & & $\downarrow$ & ND \\
\hline 23038095 & $M$ & & $\uparrow$ & $\uparrow$ \\
\hline 23042949 & $M$ & & $\downarrow$ & $\downarrow$ \\
\hline 23108651 & $M$ & Ifng $\downarrow$ & $\downarrow$ & $\downarrow$ \\
\hline 23117837 & $M$ & Pde5a $\downarrow$ & $\downarrow$ & $\downarrow$ \\
\hline 23129588 & M & $G c / m \downarrow$ & $\uparrow$ & $\uparrow$ \\
\hline 23142711 & $\mathrm{R}$ & & - & $\downarrow$ \\
\hline 23144312 & M & Mif $\downarrow$ & $\uparrow$ & $\uparrow$ \\
\hline
\end{tabular}


Westphal*

Westphal*

Yu*

Yu*

Yu*

Morgan*

Morgan*

Sun

Manso

Okamoto

Yang

Fujita

Wang

Duerrschmid*

Dai

Tamaki*

Tamaki*

Zouein

Suzuki*

Suzuki*

Engebretsen

Zhou

Chen*

Chen*

Rifki

Zhang

Park

Jiang*

Jiang*

Karakikes cardiac hypertrophy.

Postnatal ablation of Foxm1 from cardiomyocytes causes late onset hypertrophy and fibrosis whitout Exacerbating Pressure Overload-Induced Cardiac Remodeling Olmesartan attenuates cardiac remodeling through DLL4/Notch1 pathway activation in pressure overload mice.

Nampt secreted from cardiomyocytes promotes development of cardiac hypertrophy and adverse ventricular remodeling.

Effect of oxymatrine, the active component from Radix Sophorae flavescentis (Kushen), on ventricular remodeling in spontaneously hypertensive rats.

Baicalein protects against cardiac hypertrophy through blocking MEK-ERK1/2 signaling.

Effects of estrogen, an ER $\alpha$ agonist and raloxifene on pressure overload induced cardiac hypertrophy. cardiac hypertrophy.

Effects of estrogen, an ER $\alpha$ agonist and raloxifene on pressure overload induced cardiac hypertrophy.

Genetic and pharmacological inhibition of galectin-3 prevents cardiac remodeling by interfering with myocardial fibrogenesis.

Genetic and pharmacological inhibition of galectin-3 prevents cardiac remodeling by interfering with myocardial fibrogenesis.

Genetic and pharmacological inhibition of galectin-3 prevents cardiac remodeling by interfering with myocardial fibrogenesis.

Soluble epoxide hydrolase inhibition does not prevent cardiac remodeling and dysfunction after aortic constriction in rats and mice.

Soluble epoxide hydrolase inhibition does not prevent cardiac remodeling and dysfunction after aortic constriction in rats and mice.

Bone morphogenetic protein-4 mediates cardiac hypertrophy, apoptosis, and fibrosis in experimentally pathological cardiac hypertrophy.

Talin1 has unique expression versus talin 2 in the heart and modifies the hypertrophic response to pressure overload.

FHL2 prevents cardiac hypertrophy in mice with cardiac-specific deletion of ROCK2. Inhibition of farnesyl pyrophosphate synthase attenuates angiotensin II-induced cardiac hypertrophy and fibrosis in vivo.

Atrial natriuretic peptide exerts protective action against angiotensin II-induced cardiac remodeling by attenuating inflammation via endothelin-1/endothelin receptor A cascade.

Reduced cardiac fructose 2,6 bisphosphate increases hypertrophy and decreases glycolysis following aortic constriction.

Apocynin attenuates oxidative stress and cardiac fibrosis in angiotensin II-induced cardiac diastolic dysfunction in mice.

TNF receptor 1 signaling is critically involved in mediating angiotensin-II-induced cardiac fibrosis

TNF receptor 1 signaling is critically involved in mediating angiotensin-II-induced cardiac fibrosis

IKKi Deficiency Promotes Pressure Overload-Induced Cardiac Hypertrophy and Fibrosis

Metastasis-associated protein, S100A4 mediates cardiac fibrosis potentially through the modulation of $\mathrm{p} 53$ in cardiac fibroblasts.

Metastasis-associated protein, S100A4 mediates cardiac fibrosis potentially through the modulation of $\mathrm{p} 53$ in cardiac fibroblasts.

Role of STAT3 in angiotensin II-induced hypertension and cardiac remodeling revealed by mice lacking STAT3 serine 727 phosphorylation.

Long pentraxin PTX3 exacerbates pressure overload-induced left ventricular dysfunction.

Long pentraxin PTX3 exacerbates pressure overload-induced left ventricular dysfunction.

Decorin, lumican, and their GAG chain-synthesizing enzymes are regulated in myocardial remodeling and reverse remodeling in the mouse.

Paeoniflorin attenuates pressure overload-induced cardiac remodeling via inhibition of TGF $\beta /$ Smads and NF-KB pathways.

Vinexin-b protects against cardiac hypertrophy by blocking the Akt-dependent signalling pathway

Vinexin-b protects against cardiac hypertrophy by blocking the Akt-dependent signalling pathway

RaIGDS-dependent cardiomyocyte autophagy is required for load-induced ventricular hypertrophy.

Triptolide protects rat heart against pressure overload-induced cardiac fibrosis. Targeted ablation of the histidine-rich $\mathrm{Ca}(2+)$-binding protein $(\mathrm{HRC})$ gene is associated with abnormal SR $\mathrm{Ca}(2+)$-cycling and severe pathology under pressureoverload stress.

Role of interferon regulatory factor 4 in the regulation of pathological cardiac hypertrophy.

Role of interferon regulatory factor 4 in the regulation of pathological cardiac hypertrophy.

Therapeutic cardiac-targeted delivery of miR-1 reverses pressure overload-induced cardiac hypertrophy and attenuates pathological remodeling.

Indole-3-carbinol protects against pressure overload induced cardiac remodeling

\begin{tabular}{|c|c|c|c|c|}
\hline 23144938 & M & Foxm1 $\downarrow$ & - & ND \\
\hline 23188126 & $\mathrm{M}$ & & $\downarrow$ & $\downarrow$ \\
\hline 23203961 & $\mathrm{M}$ & Nampt $\downarrow$ & $\downarrow$ & $\downarrow$ \\
\hline 23211799 & $\mathrm{R}$ & & $\downarrow$ & $\downarrow$ \\
\hline 23225340 & $\mathrm{M}$ & & $\downarrow$ & $\downarrow$ \\
\hline 23227210 & $\mathrm{M}$ & Esr1 个 & - & $\downarrow$ \\
\hline 23227210 & $M$ & Esr1 个 & - & $\downarrow$ \\
\hline 23227210 & $\mathrm{M}$ & Esr1 个 & - & - \\
\hline 23230309 & $\mathrm{M}$ & Gal3 $\downarrow$ & - & $\downarrow$ \\
\hline 23230309 & $\mathrm{M}$ & Gal3 $\downarrow$ & - & $\downarrow$ \\
\hline 23230309 & $\mathrm{R}$ & Gal3 $\downarrow$ & - & $\downarrow$ \\
\hline 23232840 & $M$ & & - & - \\
\hline 23232840 & $\mathrm{R}$ & & - & - \\
\hline 23248151 & $\mathrm{M}$ & $B m p 4 \downarrow$ & $\downarrow$ & $\downarrow$ \\
\hline 23266827 & $\mathrm{M}$ & Talin1 $\downarrow$ & $\downarrow$ & $\downarrow$ \\
\hline 23271052 & $M$ & Rock2 $\downarrow$ & $\downarrow$ & $\downarrow$ \\
\hline 23277274 & $M$ & $F d p s \downarrow$ & $\downarrow$ & $\downarrow$ \\
\hline 23277455 & $\mathrm{R}$ & Nppa $\uparrow$ & - & $\downarrow$ \\
\hline 23308291 & $M$ & $P f k f b 2 \downarrow$ & $\uparrow$ & $\uparrow$ \\
\hline 23334241 & $\mathrm{M}$ & & $\downarrow$ & $\downarrow$ \\
\hline 23337087 & $\mathrm{M}$ & Tnfrsf $1 a \downarrow$ & $\downarrow$ & $\downarrow$ \\
\hline 23337087 & $\mathrm{M}$ & Tnfrsf $1 b \downarrow$ & - & - \\
\hline 23349709 & $\mathrm{M}$ & Ikbke $\downarrow$ & $\uparrow$ & $\uparrow$ \\
\hline 23352991 & $M$ & $S 100 a 4 \downarrow$ & - & $\downarrow$ \\
\hline 23352991 & $\mathrm{M}$ & S100a4 $\downarrow$ & - & $\downarrow$ \\
\hline 23364341 & $M$ & 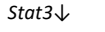 & $\downarrow$ & - \\
\hline 23372656 & $\mathrm{M}$ & Ptx3 $\downarrow$ & $\downarrow$ & $\downarrow$ \\
\hline 23372656 & $\mathrm{M}$ & Ptx3 个 & $\uparrow$ & $\uparrow$ \\
\hline 23412898 & $M$ & & ND & ND \\
\hline 23417833 & $\mathrm{M}$ & & $\downarrow$ & $\downarrow$ \\
\hline 23429936 & $\mathrm{M}$ & Sorbs $3 \downarrow$ & $\uparrow$ & $\uparrow$ \\
\hline 23429936 & $\mathrm{M}$ & Sorbs3 $\uparrow$ & $\downarrow$ & ND \\
\hline 23473774 & $\mathrm{M}$ & Ralgds $\downarrow$ & $\downarrow$ & ND \\
\hline 23541609 & $\mathrm{R}$ & & - & $\downarrow$ \\
\hline 23553082 & $M$ & $\operatorname{Hrc} \downarrow$ & $\uparrow$ & $\uparrow$ \\
\hline 23589561 & $\mathrm{M}$ & $\operatorname{Irf} 4 \downarrow$ & $\downarrow$ & $\downarrow$ \\
\hline 23589561 & $\mathrm{M}$ & Irf4 个 & $\uparrow$ & ND \\
\hline 23612897 & $\mathrm{R}$ & Mir1b 个 & $\downarrow$ & $\downarrow$ \\
\hline 23625645 & $\mathrm{M}$ & & $\downarrow$ & $\downarrow$ \\
\hline
\end{tabular}




\begin{tabular}{|c|c|c|c|c|c|c|c|c|}
\hline Ganesan & 2013 & $\begin{array}{l}\text { MiR-378 controls cardiac hypertrophy by combined repression of mitogen- } \\
\text { activated protein kinase pathway factors. }\end{array}$ & 23625957 & $M$ & Mir378 个 & $\downarrow$ & $\downarrow$ & ND \\
\hline Deng & 2013 & $\begin{array}{l}\text { Hesperetin protects against cardiac remodelling induced by pressure overload in } \\
\text { mice. }\end{array}$ & 23719775 & $M$ & & $\downarrow$ & $\downarrow$ & ND \\
\hline Misaka & 2013 & $\begin{array}{l}\text { Deficiency of senescence marker protein } 30 \text { exacerbates angiotensin II-induced } \\
\text { cardiac remodelling. }\end{array}$ & 23723062 & $\mathrm{M}$ & $\operatorname{Rgn} \downarrow$ & $\uparrow$ & $\uparrow$ & ND \\
\hline Chen & 2013 & $\begin{array}{l}\text { Intermedin suppresses pressure overload cardiac hypertrophy through activation } \\
\text { of autophagy. }\end{array}$ & 23737997 & $M$ & & $\downarrow$ & $\downarrow$ & ND \\
\hline Liu & 2013 & $\begin{array}{l}\text { A Novel Immunomodulator, FTY- } 720 \text { Reverses Existing Cardiac Hypertrophy and } \\
\text { Fibrosis From Pressure Overload by Targeting NFAT (Nuclear Factor of Activated T- } \\
\text { cells) Signaling and Periostin }\end{array}$ & 23753531 & $\mathrm{M}$ & & $\downarrow$ & $\downarrow$ & $\downarrow$ \\
\hline Wei & 2013 & Smad7 inhibits angiotensin II-induced hypertensive cardiac remodelling. & 23761400 & M & Smad7 $\uparrow$ & $\downarrow$ & $\downarrow$ & $\downarrow$ \\
\hline Jiang & 2013 & $\begin{array}{l}\text { Role for granulocyte colony stimulating factor in angiotensin II-induced neutrophil } \\
\text { recruitment and cardiac fibrosis in mice. }\end{array}$ & 23761490 & M & $\operatorname{Csf3} \downarrow$ & $\downarrow$ & $\downarrow$ & $\downarrow$ \\
\hline Ikeda & 2013 & $\begin{array}{l}\text { Deletion of phd } 2 \text { in myeloid lineage attenuates hypertensive cardiovascular } \\
\text { remodeling. }\end{array}$ & 23778187 & M & $E g \ln 1 \downarrow$ & $\downarrow$ & $\downarrow$ & $\downarrow$ \\
\hline $\mathrm{Li}^{*}$ & 2013 & $\begin{array}{l}\text { Alpha-calcitonin gene-related peptide is protective against pressure overload- } \\
\text { induced heart failure }\end{array}$ & 23816470 & M & Calca $\downarrow$ & $\uparrow$ & $\uparrow$ & $\uparrow$ \\
\hline $\mathrm{Li}^{*}$ & 2013 & $\begin{array}{l}\text { Alpha-calcitonin gene-related peptide is protective against pressure overload- } \\
\text { induced heart failure }\end{array}$ & 23816470 & $M$ & Calca $\downarrow$ & $\uparrow$ & $\uparrow$ & $\uparrow$ \\
\hline Wang & 2013 & $\begin{array}{l}\text { Pirfenidone attenuates cardiac fibrosis in a mouse model of TAC-induced left } \\
\text { ventricular remodeling by suppressing NLRP3 inflammasome formation. }\end{array}$ & 23839341 & M & & $\downarrow$ & $\downarrow$ & $\downarrow$ \\
\hline Tanaka & 2013 & $\begin{array}{l}\text { The overexpression of Twinkle helicase ameliorates the progression of cardiac } \\
\text { fibrosis and heart failure in pressure overload model in mice. }\end{array}$ & 23840758 & $M$ & Peo1 $\uparrow$ & - & $\downarrow$ & ND \\
\hline Zhang & 2013 & $\begin{array}{l}\text { Fibulin- } 2 \text { deficiency attenuates angiotensin II-induced cardiac hypertrophy by } \\
\text { reducing transforming growth factor- } \beta \text { signalling. }\end{array}$ & 23841699 & $M$ & $F b / n 2 \downarrow$ & $\downarrow$ & $\downarrow$ & ND \\
\hline Jia & 2013 & $\begin{array}{l}\text { Inhibition of platelet activation by clopidogrel prevents hypertension-induced } \\
\text { cardiac inflammation and fibrosis. }\end{array}$ & 23887740 & M & & $\downarrow$ & $\downarrow$ & $\downarrow$ \\
\hline Wei & 2013 & $\begin{array}{l}\text { Deficiency of Smad7 enhances cardiac remodeling induced by angiotensin II } \\
\text { infusion in a mouse model of hypertension. }\end{array}$ & 23894614 & $M$ & Smad7 $\downarrow$ & $\uparrow$ & $\uparrow$ & $\uparrow$ \\
\hline Konstandin & 2013 & $\begin{array}{l}\text { Fibronectin contributes to pathological cardiac hypertrophy but not physiological } \\
\text { growth. }\end{array}$ & 23912225 & M & $F n 1 \downarrow$ & $\downarrow$ & $\downarrow$ & ND \\
\hline Heymans* & 2013 & Macrophage microRNA-155 promotes cardiac hypertrophy and failure. & 23956210 & M & Mir155 $\downarrow$ & $\downarrow$ & - & $\downarrow$ \\
\hline Heymans* & 2013 & Macrophage microRNA-155 promotes cardiac hypertrophy and failure. & 23956210 & M & Mir155 $\downarrow$ & $\downarrow$ & - & $\downarrow$ \\
\hline Zong & 2013 & $\begin{array}{l}\text { NOD2 deletion promotes cardiac hypertrophy and fibrosis induced by pressure } \\
\text { overload }\end{array}$ & 23958879 & M & Nod $2 \downarrow$ & $\uparrow$ & $\uparrow$ & ND \\
\hline Pedram* & 2013 & $\begin{array}{l}\text { ER } \beta \text { selective agonist inhibits angiotensin-induced cardiovascular pathology in } \\
\text { female mice. }\end{array}$ & 23970786 & M & $E s r 1 \downarrow$ & - & - & ND \\
\hline Pedram* & 2013 & $\begin{array}{l}\text { ER } \beta \text { selective agonist inhibits angiotensin-induced cardiovascular pathology in } \\
\text { female mice. }\end{array}$ & 23970786 & $M$ & Esr1 $\uparrow$ & $\downarrow$ & $\downarrow$ & ND \\
\hline Windak & 2013 & $\begin{array}{l}\text { The AP- } 1 \text { transcription factor c-Jun prevents stress-imposed maladaptive } \\
\text { remodeling of the heart. }\end{array}$ & 24039904 & M & Jun $\downarrow$ & - & $\uparrow$ & ND \\
\hline Jiang* & 2013 & $\begin{array}{l}\text { Interferon regulatory factor } 9 \text { protects against cardiac hypertrophy by targeting } \\
\text { myocardin }\end{array}$ & 24144649 & $M$ & $\operatorname{Irf} 9 \downarrow$ & $\uparrow$ & $\uparrow$ & ND \\
\hline Jiang* & 2013 & $\begin{array}{l}\text { Interferon regulatory factor } 9 \text { protects against cardiac hypertrophy by targeting } \\
\text { myocardin }\end{array}$ & 24144649 & M & $\operatorname{Irf} 9 \downarrow$ & $\uparrow$ & $\uparrow$ & ND \\
\hline Jiang* & 2013 & $\begin{array}{l}\text { Interferon regulatory factor } 9 \text { protects against cardiac hypertrophy by targeting } \\
\text { myocardin }\end{array}$ & 24144649 & $M$ & $\operatorname{Irfg} \uparrow$ & $\downarrow$ & $\downarrow$ & ND \\
\hline Jiang* & 2013 & $\begin{array}{l}\text { Interferon regulatory factor } 9 \text { protects against cardiac hypertrophy by targeting } \\
\text { myocardin }\end{array}$ & 24144649 & $M$ & $\operatorname{Irfg} \uparrow$ & $\downarrow$ & $\downarrow$ & ND \\
\hline Higashikuni & 2013 & $\begin{array}{l}\text { Toll-like receptor- } 2 \text { mediates adaptive cardiac hypertrophy in response to pressure } \\
\text { overload through interleukin-1B upregulation via nuclear factrok kB activation }\end{array}$ & 24249711 & M & $T / r 2 \downarrow$ & $\downarrow$ & $\downarrow$ & $\downarrow$ \\
\hline Netsu & 2014 & Midkine exacerbates pressure overload-induced cardiac remodeling & 24291499 & M & $M d k \uparrow$ & $\uparrow$ & $\uparrow$ & ND \\
\hline Liu & 2014 & Regulator of G protein signaling 3 protects against cardiac hypertrophy in mice. & 24375609 & M & RGS3 个 & $\downarrow$ & $\downarrow$ & ND \\
\hline Jiang* & 2014 & $\begin{array}{l}\text { Interferon regulatory factor } 7 \text { functions as a novel negative regulator of } \\
\text { pathological cardiac hypertrophy. }\end{array}$ & 24396025 & M & Irf7 $\downarrow$ & $\uparrow$ & $\uparrow$ & $\uparrow$ \\
\hline Jiang* & 2014 & $\begin{array}{l}\text { Interferon regulatory factor } 7 \text { functions as a novel negative regulator of } \\
\text { pathological cardiac hypertrophy. }\end{array}$ & 24396025 & M & $\operatorname{lrf} 7 \uparrow$ & $\downarrow$ & $\downarrow$ & $\downarrow$ \\
\hline Zhang* & 2014 & Dickkopf-3 attenuates pressure overload-induced cardiac remodelling. & 24413772 & M & $D k k 3 \downarrow$ & $\uparrow$ & $\uparrow$ & ND \\
\hline Zhang* & 2014 & Dickkopf-3 attenuates pressure overload-induced cardiac remodelling. & 24413772 & M & Dkk3 个 & $\downarrow$ & $\downarrow$ & ND \\
\hline van Bilsen & 2014 & $\begin{array}{l}\text { Hypertension is a conditional factor for the development of cardiac hypertrophy in } \\
\text { type } 2 \text { diabetic mice. }\end{array}$ & 24416343 & $M$ & & $\uparrow$ & - & - \\
\hline Zhang & 2014 & $\begin{array}{l}\text { Antagonist of C5aR prevents cardiac remodeling in angiotensin II-induced } \\
\text { hypertension. }\end{array}$ & 24419904 & $M$ & $H c \downarrow$ & $\downarrow$ & $\downarrow$ & $\downarrow$ \\
\hline Wang & 2014 & $\begin{array}{l}\text { Double-stranded RNA-dependent protein kinase deficiency protects the heart from } \\
\text { systolic overload-induced congestive heart failure. }\end{array}$ & 24463368 & $M$ & Eif2ak2 $\downarrow$ & - & $\downarrow$ & $\downarrow$ \\
\hline Maejima* & 2014 & $\begin{array}{l}\text { Muscle-specific RING finger } 1 \text { negatively regulates pathological cardiac hypertrophy } \\
\text { through downregulation of calcineurin A. }\end{array}$ & 24526353 & $M$ & $\operatorname{Trim} 63 \downarrow$ & $\uparrow$ & ND & ND \\
\hline Maejima* & 2014 & $\begin{array}{l}\text { Muscle-specific RING finger } 1 \text { negatively regulates pathological cardiac hypertrophy } \\
\text { through downregulation of calcineurin A. }\end{array}$ & 24526353 & M & Trim63 $\downarrow$ & $\uparrow$ & $\uparrow$ & ND \\
\hline Pereira & 2014 & $\begin{array}{l}\text { GLUT1 deficiency in cardiomyocytes does not accelerate the transition from } \\
\text { compensated hypertrophy to heart failure. }\end{array}$ & 24583251 & $M$ & $S / c 2 a 1 \downarrow$ & $\uparrow$ & - & ND \\
\hline Songstad & 2014 & $\begin{array}{l}\text { Effect of transverse aortic constriction on cardiac structure, function and gene } \\
\text { expression in pregnant rats. }\end{array}$ & 24586871 & $\mathrm{R}$ & & - & - & ND \\
\hline Jang & 2014 & $\begin{array}{l}\text { Imatinib mesylate attenuates myocardial remodeling through inhibition of platelet- } \\
\text { derived growth factor and transforming growth factor activation in a rat model of } \\
\text { hypertension. }\end{array}$ & 24591339 & $\mathrm{R}$ & & $\downarrow$ & $\downarrow$ & ND \\
\hline
\end{tabular}




\begin{tabular}{|c|c|c|c|c|c|c|c|c|}
\hline Ma* & 2014 & $\begin{array}{l}\text { The requirement of } \mathrm{CD} 8+\mathrm{T} \text { cells to initiate and augment acute cardiac } \\
\text { inflammatory response to high blood pressure. }\end{array}$ & 24600037 & $\mathrm{M}$ & $C c / 2 \downarrow$ & ND & $\downarrow$ & $\downarrow$ \\
\hline Ma* & 2014 & $\begin{array}{l}\text { The requirement of CD8+ T cells to initiate and augment acute cardiac } \\
\text { inflammatory response to high blood pressure. }\end{array}$ & 24600037 & M & $C d 8 a \downarrow$ & ND & $\downarrow$ & $\downarrow$ \\
\hline Jiang* & 2014 & $\begin{array}{l}\text { Interferon regulatory factor } 1 \text { is required for cardiac remodeling in response to } \\
\text { pressure overload. }\end{array}$ & 24732887 & $M$ & $\operatorname{Irf} 1 \downarrow$ & $\downarrow$ & $\downarrow$ & ND \\
\hline Jiang* & 2014 & $\begin{array}{l}\text { Interferon regulatory factor } 1 \text { is required for cardiac remodeling in response to } \\
\text { pressure overload. }\end{array}$ & 24732887 & $M$ & $\operatorname{Irf1} \uparrow$ & $\uparrow$ & $\uparrow$ & ND \\
\hline Jiang* & 2014 & $\begin{array}{l}\text { Interferon regulatory factor } 1 \text { is required for cardiac remodeling in response to } \\
\text { pressure overload. }\end{array}$ & 24732887 & $\mathrm{R}$ & $\operatorname{Irf} 1 \downarrow$ & $\downarrow$ & $\downarrow$ & ND \\
\hline Wang & 2014 & Nrf2 enhances myocardial clearance of toxic ubiquitinated proteins. & 24747945 & M & $N r f 2 \uparrow$ & $\downarrow$ & $\downarrow$ & ND \\
\hline Li* & 2014 & $\begin{array}{l}\text { Caspase recruitment domain } 6 \text { protects against cardiac hypertrophy in response to } \\
\text { pressure overload. }\end{array}$ & 24777975 & M & Card $6 \downarrow$ & $\uparrow$ & $\uparrow$ & ND \\
\hline Li* & 2014 & $\begin{array}{l}\text { Caspase recruitment domain } 6 \text { protects against cardiac hypertrophy in response to } \\
\text { pressure overload. }\end{array}$ & 24777975 & M & Card6个 & $\downarrow$ & $\downarrow$ & ND \\
\hline $\mathrm{Li}^{*}$ & 2014 & $\begin{array}{l}\text { Caspase recruitment domain } 6 \text { protects against cardiac hypertrophy in response to } \\
\text { pressure overload. }\end{array}$ & 24777975 & M & Card6 1 & $\downarrow$ & $\downarrow$ & ND \\
\hline Kritzer & 2014 & $\begin{array}{l}\text { The scaffold protein muscle A-kinase anchoring protein } \beta \text { orchestrates cardiac } \\
\text { myocyte hypertrophic signaling required for the development of heart failure. }\end{array}$ & 24812305 & $M$ & Akap6 $\downarrow$ & $\downarrow$ & $\downarrow$ & ND \\
\hline Bracey & 2014 & $\begin{array}{l}\text { Mitochondrial NLRP3 protein induces reactive oxygen species to promote Smad } \\
\text { protein signaling and fibrosis independent from the inflammasome. }\end{array}$ & 24841199 & M & NIrp3 $\downarrow$ & - & $\downarrow$ & ND \\
\hline Demos-Davies* & 2014 & $\begin{array}{l}\text { HDAC6 contributes to pathological responses of heart and skeletal muscle to } \\
\text { chronic angiotensin-II signaling. }\end{array}$ & 24858848 & $M$ & Hdac6 $\downarrow$ & - & - & ND \\
\hline Demos-Davies* & 2014 & $\begin{array}{l}\text { HDAC6 contributes to pathological responses of heart and skeletal muscle to } \\
\text { chronic angiotensin-II signaling. }\end{array}$ & 24858848 & M & Hdac6 $\downarrow$ & - & - & - \\
\hline $\mathrm{Li}$ & 2014 & $\begin{array}{l}\text { } \delta \mathrm{T} \text { Cell-Derived Interleukin-17A via an Interleukin-1ß-Dependent Mechanism } \\
\text { Mediates Cardiac Injury and Fibrosis in Hypertension }\end{array}$ & 24866139 & M & $\| 17 a \downarrow$ & ND & $\downarrow$ & $\downarrow$ \\
\hline Wang & 2014 & $\begin{array}{l}\text { 5TNF- } \alpha \text { and IL-1 } \beta \text { neutralization ameliorates angiotensin II-induced cardiac damage } \\
\text { in male mice. }\end{array}$ & 24877626 & M & $\begin{array}{l}\text { Tnf } \downarrow, \| 1 b \\
\downarrow\end{array}$ & $\downarrow$ & $\downarrow$ & $\downarrow$ \\
\hline Liu & 2014 & $\begin{array}{l}\text { Endoplasmic reticulum stress sensor protein kinase R-like endoplasmic reticulum } \\
\text { kinase (PERK) protects against pressure overload-induced heart failure and lung } \\
\text { remodeling. }\end{array}$ & 24958502 & M & Eif2ak3 $\downarrow$ & $\uparrow$ & $\uparrow$ & ND \\
\hline Endo & 2014 & $\begin{array}{l}\text { 18-HEPE, an } n-3 \text { fatty acid metabolite released by macrophages, prevents pressure } \\
\text { overload-induced maladaptive cardiac remodeling. }\end{array}$ & 25049337 & $M$ & & $\downarrow$ & $\downarrow$ & $\downarrow$ \\
\hline Cardin* & 2014 & $\begin{array}{l}\text { Differences in cell-type-specific responses to angiotensin II explain cardiac } \\
\text { remodeling differences in C57BL/6 mouse substrains. }\end{array}$ & 25069667 & M & & - & - & $\downarrow$ \\
\hline Cardin* & 2014 & $\begin{array}{l}\text { Differences in cell-type-specific responses to angiotensin II explain cardiac } \\
\text { remodeling differences in C57BL/6 mouse substrains. }\end{array}$ & 25069667 & $M$ & & - & - & $\uparrow$ \\
\hline Patrucco & 2014 & $\begin{array}{l}\text { Roles of cGMP-dependent protein kinase I (cGKI) and PDE5 in the regulation of Ang } \\
\text { II-induced cardiac hypertrophy and fibrosis. }\end{array}$ & 25139994 & M & Prkg1 $\downarrow$ & - & - & ND \\
\hline Bernardo & 2014 & $\begin{array}{l}\text { Therapeutic silencing of miR- } 652 \text { restores heart function and attenuates adverse } \\
\text { remodeling in a setting of established pathological hypertrophy. }\end{array}$ & 25145628 & M & Mir652 $\downarrow$ & $\downarrow$ & $\downarrow$ & ND \\
\hline Yang & 2014 & $\begin{array}{l}\text { Granule cargo release from bone marrow-derived cells sustains cardiac } \\
\text { hypertrophy. }\end{array}$ & 25239803 & $M$ & $U g r 2 b \downarrow$ & $\downarrow$ & - & - \\
\hline
\end{tabular}


Chapter 6

General Discussion 


\section{Inflammation ignites cardiac disease progression}

Understanding the molecular mechanism underlying cardiac disease is crucial to improve diagnosis and develop novel treatment strategies. In this context, the role of inflammation in the pathophysiology of cardiac disease, and heart failure (HF) in particular, is receiving widespread attention and it is becoming evident that inflammation plays a pivotal role in the progression of this disease ${ }^{1,2}$. Besides that, patients suffering from inflammatory pathologies with increased blood cytokine levels, such as rheumatoid arthritis, have an increased risk to develop $\mathrm{HF}^{3}$. Several key studies have shown levels of the inflammatory cytokines TNF $\alpha$, IL$1 \beta$ and IL- 6 to be elevated the blood ${ }^{4}$ as well as in the myocardium ${ }^{5}$ of HF patients. In addition, plasma concentrations of TNF $\alpha$ and IL- 6 predict survival chances in HF patients ${ }^{6}$. Collectively, these observations indicate that inflammatory mechanisms play a prominent role in disease progression in HF.

The aim of this thesis was to better understand the role of the immune system in $\mathrm{HF}$ and to explore how it can be manipulated in such way that it preserves its crucial role in beneficial processes such as wound healing and pathogen clearance, but that its destructive effects on the cardiac tissue are diminished. All four experimental chapters of this thesis have touched upon this goal in different ways and will be discussed in the context of inflammation in this chapter.

Since the role of inflammation in the disease progression of HF was first described several decades ago ${ }^{7}$, pre-clinical papers have appeared in which immuno-modulatory approaches were tested in animal models of HF. At the same time, in the mid-nineties, the wide availability of knockout (KO) and transgenic (TG) mice increased the toolset available to manipulate the inflammatory response. The first pre-clinical papers to elaborate on inflammation in HF focussed on the role of TNF ${ }^{8-10}$, considered to be one of the most important pro-inflammatory cytokines. Swiftly, the focus broadened to other proinflammatory effectors, including numerous cytokines and chemokines. For instance, treatment of hypertensive rats with a MCP-1 neutralising anti-body resulted in improvement of cardiac diastolic function ${ }^{11}$. Subsequently more studies showed that modulation of inflammatory processes influences cardiac pathology upon pressure overload (PO) induced $\mathrm{HF}^{12-16}$.

Cardiac stress results in myocardial inflammation, reflected by infiltration of multiple immune cell populations into the myocardium ${ }^{5}$. The synthesis and release of chemokines by cardiac cells attracts myeloid cells like monocytes, macrophages 17, 18, neutrophils ${ }^{18}$, as well as lymphocytes ${ }^{1}$ to the heart. Each of these leukocyte populations exerts specific effects on the pathophysiological processes underlying HF development ${ }^{1}$. Moreover, these cell types are bearing specific subtypes that can either be pro- or antiinflammatory, and therefore harmful or beneficial in disease progression. For instance, Tregulatory cells have been shown to ameliorate Angll induced cardiac damage ${ }^{19}$ and inhibit cardiac fibrosis after TAC ${ }^{20}$. In contrast, another lymphocyte cell type, Th17, was shown to be deleterious in cardiac disease ${ }^{21}$. Studies in both patients and animals indicated a relationship between pro-inflammatory markers and pressure-overload induced hypertrophy and fibrosis ${ }^{5}$. The question is if immune activation per se is detrimental in the chronically pressure overloaded heart. In this thesis we focussed on the role of inflammation in two different HF disease aetiologies, hypertensive heart disease (HHD) and viral myocarditis (VM). HHD causes pressure overloading (PO) of the heart and ultimately leads to HF and is associated with chronic, low-grade inflammation. VM, however, is caused by a viral infection of the heart and initiates an acute and severe cardiac inflammatory response. 


\section{An adverse role for CD40 activation in the pressure-overloaded heart}

An important effector of the immune system, the CD40 receptor, was left unstudied in the context of HHD induced HF. Our study, described in chapter two, shows that the absence of the co-stimulatory molecule receptor CD40 in myeloid cells blunts cardiac hypertrophy upon Angiotensin-II (AngII) infusion. Thereby we have shown once more that intervening with the cardiac immune response is a potential way to inhibit the progression of cardiac disease. Furthermore, we have shown involvement of both CD40 downstream signalling pathways using TRAF2/3/5 and TRAF6 in the development of cardiac hypertrophy. Both these pathways are known to activate the classical NFKB pathway ${ }^{22}$, previously described as a crucial mediator of cardiac hypertrophy ${ }^{23-25}$.

An interesting finding is that deletion of CD40 reduced hypertrophy, but not fibrosis, suggesting that separate pathways are responsible for these two processes. Previously, it was shown that alternatively activated (M2) macrophages can induce fibrosis via TGF $\beta$ secretion ${ }^{26}$. The deletion of CD40 induces more alternatively activated (M2) macrophages ${ }^{27}$, that in turn, induce more fibrosis, possibly counteracting the reduction in fibrosis associated with a reduction in disease phenotype in the CD40 KO mice.

Interestingly, the ligand of the CD40 receptor, CD40 ligand (CD40L), has become an established biomarker of $\mathrm{HF}$ in hypertensive patients ${ }^{28,}{ }^{29}$. But in contrast to other cardiovascular diseases such as atherosclerosis ${ }^{27}$, no functional role for CD40L-CD40 signalling in the development of HHD was described and we are the first to report on these effects. In addition, blocking CD40L-CD40 interactions has already been subject of clinical trials in inflammatory diseases ${ }^{30}$ and a specific compound blocking CD40-TRAF6 signalling has been developed ${ }^{31,32}$. This gives opportunities for targeting CD40L-CD40 signalling as therapeutic approach for HHD.

Our research on the CD40 receptor in HHD still leaves several questions unanswered. For example, it remains unclear what paracrine factors secreted from CD40activated macrophages promote myocyte hypertrophy. Detailed proteomic analysis of the sectretome of activated $\mathrm{KO}$ and Wild type (Wt) macrophages could answer this question. Alternatively, miRNAs secreted in exosomes by these myeloid cells could be responsible for the pro-hypertrophic effect. This type of inter-cellular signalling using miRNAs has been shown previously between cardiac fibroblasts and cardiomyocytes ${ }^{33}$, where miR-21-3p derived from exosomes of fibroblasts induced hypertrophy in cardiomyocytes ${ }^{33}$.

The differences between Angll infusion and other models inducing cardiac PO, such as transverse aortic constriction (TAC), should be considered also. The pharmacological model of Angll infusion induced marked hypertension in our study, but hypertrophy induced in this model may not be limited to pressure overloading alone, as Angll can stimulate cardiomyocyte hypertrophy directly. Moreover, previously described systemic proinflammatory effects of Angll may play a role as well ${ }^{34}$. A surgical model like TAC induces PO with more local effects only. Consequently, the Angll model mimics HHD in patients, whilst TAC represents better aortic stenosis in otherwise healthy patients. 


\section{Metabolic changes during acute cardiac inflammation in viral myocarditis}

In chapter three of thesis we demonstrated that acute and fulminant inflammation in itself leads to perturbations in cardiac metabolism. Our data show that acute VM, in both humans and mice, is associated with extensive metabolic remodelling of the heart. These findings provide evidence that suppression of cardiomyocyte mitochondrial oxidative metabolism is controlled by inflammatory mechanisms, and here specifically by activation of the inflammatory NFKB pathway. In this mechanism paracrine signalling by cytokines secreted from leukocytes that infiltrate the myocardium plays a crucial role (Figure 1).

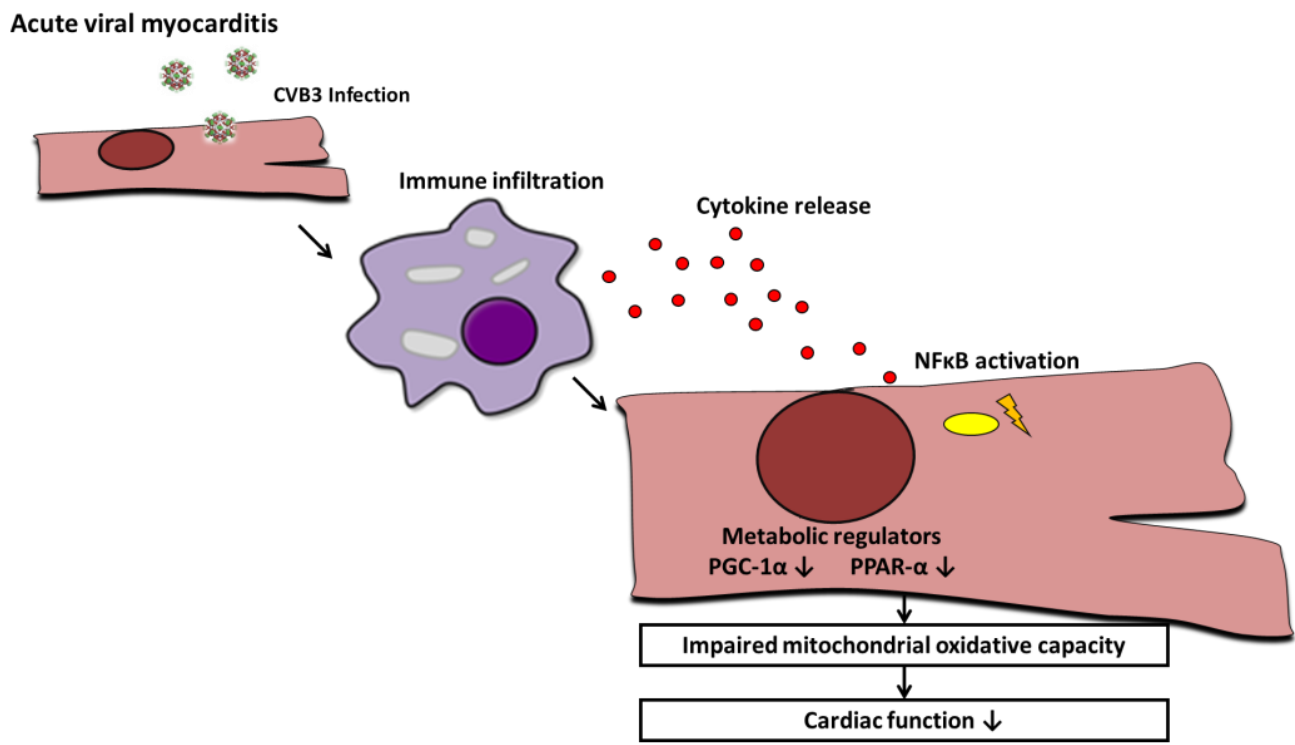

Figure 1. Representation of our findings on the effects of acute inflammation on the metabolic phenotype of the heart. Upon infection with CVB3 virus monocytes and macrophages infiltrate the heart and secrete pro-inflammatory cytokines that, in turn, activate NFKB in cardiomyocytes. NFKB inhibits the expression and activity of metabolic regulators PGC-1 $\alpha$ and PPARs. This leads to impaired mitochondrial oxidative capacity and reduced cardiac function.

To investigate if a metabolic therapy would ameliorate VM-induced cardiac injury, we treated mice with dichloroacetate (DCA), a drug known to stimulate glucose oxidation via the activation of the pyruvate dehydrogenase (PDH) complex. Previously, it was demonstrated that DCA improved cardiac function and survival in a rat model of HHD ${ }^{35}$. Strikingly, in our VM model DCA worsened the VM phenotype as reflected by a marked increase in leukocyte infiltration. Notably, administration of DCA to these animals also increased spleen weight indicative of immune-modulatory effects of DCA. This is in line with literature showing that DCA modulates the immune response by skewing macrophage polarisation towards M1 and inducing T-helper 1 lymphocyte function ${ }^{36}$. These immune-modulatory effects of DCA may override the beneficial effects of DCA on cardiac metabolism. It is unclear how this increase in inflammation affects the function of the heart at later stages of the disease. Possibly, increased M1 and Th1 function benefits the anti-viral response in the heart, allowing complete viral removal and therefore complete recovery. On the other hand, pronounced inflammation induces more irreversible tissue damage, ultimately leading to the development of dilated cardiomyopathy (DCM).

This brings us to the general question on how inflammation in VM should be understood. Different mouse strains show variable susceptibility to CVB3-induced VM, 
possibly due to differences in their immuno-genetic background. Likewise, some people develop a myocarditis during a CVB3 or PVB19 infection while the majority does not. Also here the immuno-genetic background, gender, previous infections and other factors may play a role ${ }^{37}$. Patients that do develop myocarditis sometimes completely recover, leaving no visible damage to the heart, while other patients develop dilated cardiomyopathy over time. In this light, the question can be posed whether inflammation is beneficial or harmful in CVB3-induced VM. Most likely, pronounced pro-inflammatory activation does more harm than good to the myocardial tissue, but is an inevitable consequence of the viral infection in the heart ${ }^{37}$.

An interesting question is, if inflammation is inducing adverse perturbations in cardiac metabolism, why this mechanism has evolved? It is likely that these metabolic perturbations serve a function in the immune response. Previously, macrophage-mediated inflammation and insulin resistance were shown to be linked ${ }^{38}$. This crosstalk between the immune system and metabolism is conserved among species ${ }^{39}$ suggesting that inflammation induced metabolic perpetuations are beneficial under specific circumstances. One such scenario is an acute infection, where it provides a mechanism for diverting nutrients away from non-essential functions, such as storage, towards immunity by raising glucose levels to "fuel" the highly glycolic leukocytes ${ }^{40}$. In this way metabolic changes can aid the antipathogen response.

\section{What distinguishes adverse from beneficial inflammation during HF?}

The results presented in chapter two and three of this thesis suggest that inflammation is playing a deleterious role in $\mathrm{HF}$, and that targeting the cardiac immune response is a good strategy to prevent disease progression. Finding a therapy that effectively reduces the intensity and duration of the immune response, without interfering with wound healing and reparative mechanisms remains a challenge. Although there have been several clinical trials addressing this (see ${ }^{41}$ for review), no such adequate therapy has yet emerged. The difficulty to discriminate between beneficial and harmful inflammation and the pleiotropic effects of many cytokines are possible reasons for this. For instance the cytokine IL- 4 is considered to have anti-inflammatory effects, but at the same time was shown stimulate fibrosis ${ }^{42}$.

In the heart a competent cardiac immune response is mounted after acute damage induced by myocardial infarction (MI). Necrotic and damaged cardiomyocytes release DAMPs and are in turn cleared by phagocytic cells. In the overloaded heart it is less clear what initiates the inflammatory response. There are indications that mitochondria become damaged in the pressure-overloaded heart and are degraded by autophagy ${ }^{43}$. A recent study by Oka et al. ${ }^{44}$ shows that mtDNA from these stressed mitochondria escapes autophagy and is recognized by Toll-like receptor (TLR) 9, thereby initiating cytokine release. Haemodynamic stress is not limited to cardiac disease but also occurs during exercise ${ }^{45}$. In this context it is worth mentioning that endurance athletes who develop physiological hypertrophy, also show increased IL- 6 levels in the blood after a bout of exercise ${ }^{46}$. Moreover, it has been shown that activation of the NFKB pathway is needed for development of physiological cardiac hypertrophy in mice ${ }^{47}$. Considering this, it is unlikely that elevated cytokine levels per se initiate adverse cardiac remodelling during $\mathrm{PO}$, but rather their continuous presence induced by the sustained haemodynamic stress ${ }^{5}$. This continuous pro-inflammatory activation prevents resolving of inflammation and leads to accumulation of leukocytes in the heart. Elimination of these deleterious immune populations from the myocardium could be achieved by preventing their migration, or by skewing their polarisation towards anti- 
inflammatory phenotypes. In this perspective intervening in CD40 signalling seems a smart strategy, as it was shown previously to affect the infiltration of leukocytes ${ }^{30}$, macrophage polarisation ${ }^{27,48}$ and activation of lymphocytes ${ }^{30}$.

\section{The complex immune response of the heart: Alternative research approaches needed?}

Because cardiac inflammation is such a complex process, it is difficult to draw conclusions from studies that manipulate one single effector by for example knockdown of a specific cytokine or chemokine. Moreover, the redundancy in function of many cytokines and chemokines ${ }^{49}$, possibly limits the effect of these single gene $\mathrm{KO}$ studies on the disease phenotype. Indeed, in most cases HF is not a single loss-of-function genetic abnormality. Therefore, a better approach might be to target miRNAs instead of protein coding genes. Because miRNAs target multiple genes involved in pathophysiological pathways and repress, but not completely ablate transcript levels of their targets ${ }^{50}$, they exert more subtle effects. Therefore, miRNAs are able to fine-tune molecular processes involved in HF development, including inflammation. A disadvantage of the use of miRNAs is that the high number of targets per miRNA makes potential side-effects difficult to predict.

In chapter four of this thesis a high-throughput phenotypical screening was applied to explore the effect of $194 \mathrm{HF}$-associated miRNAs on three pivotal cell-type specific processes underlying HF development, namely hypertrophy, fibrosis, and inflammation ${ }^{51}$. This comprehensive screening approach unveiled the effects of various miRNAs in cardiovascular disease and opened new avenues for the treatment of HF via miRNA-directed therapeutic approaches. By performing this screen in three cell types, we discovered novel roles for miRNAs in hypertrophy, fibrosis and inflammation, and we confirmed the effects of well-known "inflammatory" miRNAs such as miR-155 ${ }^{17,52}$. Additionally, we identified miRNAs new to the HF research field. Several miRNAs affected all of the pathophysiological processes investigated. For example, transfection with mimics of miR-200c-3p and miR-210-3p increased cell size in cardiac myocytes (hypertrophy), increased collagen content in cardiac fibroblasts (fibrosis), and it skewed macrophages towards M1 polarization. MiR-891a-3p mimics, on the other hand, influence these processes in the other, more beneficial direction. Both these results open up therapeutic opportunities in HF, either by blocking (miR-200c-3p, miR-210-3p) or delivering (miR-891a-3p) these miRNAs in vivo.

\section{New insights using published pre-clinical studies}

Animal models are vital for advancement of our understanding of the pathophysiology of HF and therefore the development of novel therapies. With the wide availability of KO and TG mice a plethora of studies addressing the molecular pathways of HF has been published over the past decades (chapter five of this thesis). Although many of these studies have shown promising results in the protection against HF in animal models, disappointingly, success in the translation of these studies into clinical practice remains limited ${ }^{2}$. In combination, however, these pre-clinical studies are the puzzle pieces that give clues about the complex molecular and cellular pathways involved in the pathogenesis of HF. In chapter five we have combined almost 300 of these studies, thereby providing an unprecedented overview of preclinical cardiac PO studies published in the last 15 years. Using this dataset, we could show that inflammation closely correlates with the pathophysiological processes of hypertrophy and fibrosis and that, whenever the degree of hypertrophy or fibrosis changes, there is a parallel change in the degree of inflammation. Conversely, actively decreasing or increasing inflammation by targeting genes involved in the molecular processes underlying 
inflammation, almost always leads to a comparable change in hypertrophy and fibrosis. Previously, network analysis has been used to link expression levels of messenger RNA and microRNAs in dilated cardiomyopathy (DCM) in a miRNA-Gene-Network ${ }^{53}$. In chapter five we have constructed a gene to phenotype network (GPIN) based on our systematic review of preclinical studies. Using published data, we determined the effect of individual genes on, what we consider, key pathophysiological processes in HF. This network was extended with miRNAs predicted to target the genes that are linked to phenotypes. By using a scoring system for miRNAs targeting these genes, we identified several miRNAs as crucial regulators of pathophysiological processes underlying HF disease progression. The fact that the relatively unknown miR-466I-3p turned out to be the miRNA with the highest cumulative impact on the three outcome parameters, warrants further investigation into its role in PO induced HF. We also identified miR-340, previously identified in DCM patients by network analysis ${ }^{53}$, to be involved in PO-induced HF. Interestingly, several miRNAs identified in chapter five also displayed pronounced phenotypes in the in vitro the screen described in chapter four, thereby conforming their importance in HF disease progression once more. Transfection with miR-19a-3p induced significant increases in all readouts, except NFkB nuclear translocation. MiR-340-5p significantly increases macrophage roundness and decreases both the number of fibroblasts as well as cardiomyocyte cell size. In conclusion, the meta-analysis-based GPIN has proven to be an insightful novel approach to generate new "knowledge" from published data and awaits future application to other animal models of human cardiovascular disease.

\section{Conclusion and future perspectives}

The research in this thesis has led to a better understanding of the immune response during $\mathrm{HF}$ and created several openings on how to manipulate this response. In chapter two we show that CD40L-CD40-TRAF signalling modulates cardiac hypertrophy. Moreover, we provided additional evidence for the importance of myeloid cells in the development of cardiac hypertrophy. The CD40L-CD40 co-stimulatory dyad offers opportunities for the treatment of HHD. The study described in chapter three supports the concept that cytokines, including TNF- $\alpha$, released from inflammatory cells infiltrating the myocardium, lead to the activation of NFKB in cardiomyocytes, ultimately resulting in impairment of mitochondrial oxidative metabolism. These metabolic perturbations are likely to contribute to the cardiac contractile dysfunction during acute VM. In chapter four we performed a high throughput screen in three relevant primary cell types simultaneously, thereby identifying miRNAs involved in hypertrophy, fibrosis and inflammation. In this way, not only we confirmed roles of well-known miRNAs, but also identified many miRNAs new to the HF-research field. Finally, we established a novel approach to identify potential HF therapeutic targets in chapter five. Using existing literature, a Gene to Phenotype inte raction network was created that allowed the identification of novel miRNAs putatively involved in HF pathogenesis. The vast amount of miRNA data in this thesis is a gold-mine for future miRNA research and might ultimately lead to novel therapies. 


\section{References}

1. Frieler RA and Mortensen RM. Immune cell and other noncardiomyocyte regulation of cardiac hypertrophy and remodeling. Circulation. 2015;131:1019-30.

2. Heymans S, Hirsch E, Anker SD, Aukrust P, Balligand JL, Cohen-Tervaert JW, Drexler H, Filippatos G, Felix SB, Gullestad L, Hilfiker-Kleiner D, Janssens S, Latini R, Neubauer G, Paulus WJ, Pieske B, Ponikowski P, Schroen B, Schultheiss HP, Tschope C, Van Bilsen M, Zannad F, McMurray J and Shah AM. Inflammation as a therapeutic target in heart failure? A scientific statement from the Translational Research Committee of the Heart Failure Association of the European Society of Cardiology. Eur J Heart Fail. 2009;11:119-29.

3. Wolfe $\mathrm{F}$ and Michaud K. Heart failure in rheumatoid arthritis: rates, predictors, and the effect of antitumor necrosis factor therapy. Am J Med. 2004;116:305-11.

4. Bozkurt B, Mann DL and Deswal A. Biomarkers of inflammation in heart failure. Heart Fail Rev. 2010;15:331-41.

5. Mann DL. Inflammatory mediators and the failing heart: past, present, and the foreseeable future. Circ Res. 2002;91:988-98.

6. Rauchhaus M, Doehner W, Francis DP, Davos C, Kemp M, Liebenthal C, Niebauer J, Hooper J, Volk HD, Coats AJ and Anker SD. Plasma cytokine parameters and mortality in patients with chronic heart failure. Circulation. 2000;102:3060-7.

7. Levine B, Kalman J, Mayer L, Fillit HM and Packer M. Elevated circulating levels of tumor necrosis factor in severe chronic heart failure. N Engl J Med. 1990;323:236-41.

8. Bozkurt B, Kribbs SB, Clubb FJ, Jr., Michael LH, Didenko VV, Hornsby PJ, Seta Y, Oral H, Spinale FG and Mann DL. Pathophysiologically relevant concentrations of tumor necrosis factor-alpha promote progressive left ventricular dysfunction and remodeling in rats. Circulation. 1998;97:1382-91.

9. Kubota T, McTiernan CF, Frye CS, Slawson SE, Lemster BH, Koretsky AP, Demetris AJ and Feldman AM. Dilated cardiomyopathy in transgenic mice with cardiac-specific overexpression of tumor necrosis factoralpha. Circ Res. 1997;81:627-35.

10. Moe GW, Marin-Garcia J, Konig A, Goldenthal M, Lu X and Feng Q. In vivo TNF-alpha inhibition ameliorates cardiac mitochondrial dysfunction, oxidative stress, and apoptosis in experimental heart failure. Am J Physiol Heart Circ Physiol. 2004;287:H1813-20.

11. Kuwahara F, Kai H, Tokuda K, Takeya M, Takeshita A, Egashira K and Imaizumi T. Hypertensive myocardial fibrosis and diastolic dysfunction: another model of inflammation? Hypertension. 2004;43:739-45.

12. Duerrschmid C, Crawford JR, Reineke E, Taffet GE, Trial J, Entman ML and Haudek SB. TNF receptor 1 signaling is critically involved in mediating angiotensin-ll-induced cardiac fibrosis. J Mol Cell Cardiol. 2013;57:59-67.

13. Honsho S, Nishikawa S, Amano K, Zen K, Adachi Y, Kishita E, Matsui A, Katsume A, Yamaguchi S, Nishikawa K, Isoda K, Riches DW, Matoba S, Okigaki M and Matsubara H. Pressure-mediated hypertrophy and mechanical stretch induces IL-1 release and subsequent IGF-1 generation to maintain compensative hypertrophy by affecting Akt and JNK pathways. Circ Res. 2009;105:1149-58.

14. Haudek SB, Cheng J, Du J, Wang Y, Hermosillo-Rodriguez J, Trial J, Taffet GE and Entman ML. Monocytic fibroblast precursors mediate fibrosis in angiotensin-II-induced cardiac hypertrophy. J Mol Cell Cardiol. 2010;49:499-507.

15. Hirota H, Yoshida K, Kishimoto $\mathrm{T}$ and Taga T. Continuous activation of gp130, a signal-transducing receptor component for interleukin 6-related cytokines, causes myocardial hypertrophy in mice. Proc Natl Acad Sci U S A. 1995;92:4862-6.

16. Coles B, Fielding CA, Rose-John S, Scheller J, Jones SA and O'Donnell VB. Classic interleukin-6 receptor signaling and interleukin-6 trans-signaling differentially control angiotensin II-dependent hypertension, cardiac signal transducer and activator of transcription-3 activation, and vascular hypertrophy in vivo. The American journal of pathology. 2007;171:315-25.

17. Heymans S, Corsten MF, Verhesen W, Carai P, van Leeuwen RE, Custers K, Peters T, Hazebroek M, Stoger L, Wijnands E, Janssen BJ, Creemers EE, Pinto YM, Grimm D, Schurmann N, Vigorito E, Thum T, Stassen F, Yin X, Mayr M, de Windt LJ, Lutgens E, Wouters K, de Winther MP, Zacchigna S, Giacca M, van Bilsen M, Papageorgiou AP and Schroen B. Macrophage microRNA-155 promotes cardiac hypertrophy and failure. Circulation. 2013;128:1420-32.

18. Xia Y, Lee K, Li N, Corbett D, Mendoza L and Frangogiannis NG. Characterization of the inflammatory and fibrotic response in a mouse model of cardiac pressure overload. Histochemistry and cell biology. 2009;131:471-81. 
19. Kvakan H, Kleinewietfeld M, Qadri F, Park JK, Fischer R, Schwarz I, Rahn HP, Plehm R, Wellner M, Elitok S, Gratze P, Dechend R, Luft FC and Muller DN. Regulatory T cells ameliorate angiotensin II-induced cardiac damage. Circulation. 2009;119:2904-12.

20. Kanellakis P, Dinh TN, Agrotis A and Bobik A. CD4(+)CD25(+)Foxp3(+) regulatory T cells suppress cardiac fibrosis in the hypertensive heart. J Hypertens. 2011;29:1820-8.

21. Madhur MS, Lob HE, McCann LA, Iwakura Y, Blinder Y, Guzik TJ and Harrison DG. Interleukin 17 promotes angiotensin II-induced hypertension and vascular dysfunction. Hypertension. 2010;55:500-7.

22. Hayden MS and Ghosh S. Regulation of NF-kappaB by TNF family cytokines. Semin Immunol. 2014;26:25366.

23. Li Y, Ha T, Gao X, Kelley J, Williams DL, Browder IW, Kao RL and Li C. NF-kappaB activation is required for the development of cardiac hypertrophy in vivo. Am J Physiol Heart Circ Physiol. 2004;287:H1712-20.

24. Freund C, Schmidt-Ullrich R, Baurand A, Dunger S, Schneider W, Loser P, El-Jamali A, Dietz R, Scheidereit C and Bergmann MW. Requirement of nuclear factor-kappaB in angiotensin II- and isoproterenol-induced cardiac hypertrophy in vivo. Circulation. 2005;111:2319-25.

25. Kawano S, Kubota T, Monden Y, Kawamura N, Tsutsui H, Takeshita A and Sunagawa K. Blockade of NFkappaB ameliorates myocardial hypertrophy in response to chronic infusion of angiotensin II. CardiovasC Res. 2005;67:689-98.

26. Huber S, Schramm C, Lehr HA, Mann A, Schmitt S, Becker C, Protschka M, Galle PR, Neurath MF and Blessing $M$. Cutting edge: TGF-beta signaling is required for the in vivo expansion and immunosuppressive capacity of regulatory CD4+CD25+ T cells. J Immunol. 2004;173:6526-31.

27. Lutgens E, Lievens D, Beckers L, Wijnands E, Soehnlein O, Zernecke A, Seijkens T, Engel D, Cleutjens J, Keller AM, Naik SH, Boon L, Oufella HA, Mallat Z, Ahonen CL, Noelle RJ, de Winther MP, Daemen MJ, Biessen EA and Weber C. Deficient CD40-TRAF6 signaling in leukocytes prevents atherosclerosis by skewing the immune response toward an antiinflammatory profile. J Exp Med. 2010;207:391-404.

28. Ueland T, Aukrust P, Yndestad A, Otterdal K, Froland SS, Dickstein K, Kjekshus J, Gullestad L and Damas JK. Soluble CD40 ligand in acute and chronic heart failure. Eur Heart J. 2005;26:1101-7.

29. Yan JC, Liu PJ, Du RZ, Liang Y, Ma GS, Zhu J and Luo D. Relationship between CD40 ligand expression and B type natriuretic peptide levels in patients with chronic heart failure. Clin Chim Acta. 2008;392:17-20.

30. Engel D, Seijkens T, Poggi M, Sanati M, Thevissen L, Beckers L, Wijnands E, Lievens D and Lutgens E. The immunobiology of CD154-CD40-TRAF interactions in atherosclerosis. Semin Immunol. 2009;21:308-12.

31. van den Berg SM, Seijkens TT, Kusters PJ, Zarzycka B, Beckers L, den Toom M, Gijbels MJ, Chatzigeorgiou A, Weber C, de Winther MP, Chavakis T, Nicolaes GA and Lutgens E. Blocking CD40-TRAF6 interactions by small-molecule inhibitor 6860766 ameliorates the complications of diet-induced obesity in mice. International journal of obesity. 2015;39:782-90.

32. Chatzigeorgiou A, Seijkens T, Zarzycka B, Engel D, Poggi M, van den Berg S, van den Berg S, Soehnlein O, Winkels H, Beckers L, Lievens D, Driessen A, Kusters P, Biessen E, Garcia-Martin R, Klotzsche-von Ameln A, Gijbels M, Noelle R, Boon L, Hackeng T, Schulte KM, Xu A, Vriend G, Nabuurs S, Chung KJ, Willems van Dijk K, Rensen PC, Gerdes N, de Winther M, Block NL, Schally AV, Weber C, Bornstein SR, Nicolaes G, Chavakis $\mathrm{T}$ and Lutgens E. Blocking CD40-TRAF6 signaling is a therapeutic target in obesity-associated insulin resistance. Proc Natl Acad Sci U S A. 2014;111:2686-91.

33. Bang C, Batkai S, Dangwal S, Gupta SK, Foinquinos A, Holzmann A, Just A, Remke J, Zimmer K, Zeug A, Ponimaskin E, Schmiedl A, Yin X, Mayr M, Halder R, Fischer A, Engelhardt S, Wei Y, Schober A, Fiedler J and Thum T. Cardiac fibroblast-derived microRNA passenger strand-enriched exosomes mediate cardiomyocyte hypertrophy. J Clin Invest. 2014;124:2136-46.

34. McMaster WG, Kirabo A, Madhur MS and Harrison DG. Inflammation, immunity, and hypertensive endorgan damage. Circ Res. 2015;116:1022-33.

35. Kato T, Niizuma S, Inuzuka Y, Kawashima T, Okuda J, Tamaki Y, Iwanaga Y, Narazaki M, Matsuda T, Soga T, Kita T, Kimura T and Shioi T. Analysis of metabolic remodeling in compensated left ventricular hypertrophy and heart failure. Circulation Heart failure. 2010;3:420-30.

36. Ohashi T, Akazawa T, Aoki M, Kuze B, Mizuta K, Ito $Y$ and Inoue N. Dichloroacetate improves immune dysfunction caused by tumor-secreted lactic acid and increases antitumor immunoreactivity. Int J Cancer. 2013;133:1107-18.

37. Corsten MF, Schroen B and Heymans S. Inflammation in viral myocarditis: friend or foe? Trends Mol Med. 2012;18:426-37.

38. Chawla A, Nguyen KD and Goh YP. Macrophage-mediated inflammation in metabolic disease. Nat Rev Immunol. 2011;11:738-49.

39. Hotamisligil GS. Inflammation and metabolic disorders. Nature. 2006;444:860-7.

40. Newsholme P, Gordon S and Newsholme EA. Rates of utilization and fates of glucose, glutamine, pyruvate, fatty acids and ketone bodies by mouse macrophages. Biochem J. 1987;242:631-6. 
41. Mann DL, McMurray JJ, Packer M, Swedberg K, Borer JS, Colucci WS, Djian J, Drexler H, Feldman A, Kober L, Krum H, Liu P, Nieminen M, Tavazzi L, van Veldhuisen DJ, Waldenstrom A, Warren M, Westheim A, Zannad $\mathrm{F}$ and Fleming T. Targeted anticytokine therapy in patients with chronic heart failure: results of the Randomized Etanercept Worldwide Evaluation (RENEWAL). Circulation. 2004;109:1594-602.

42. Peng H, Sarwar Z, Yang XP, Peterson EL, Xu J, Janic B, Rhaleb N, Carretero OA and Rhaleb NE. Profibrotic Role for Interleukin-4 in Cardiac Remodeling and Dysfunction. Hypertension. 2015;66:582-9.

43. Nakai A, Yamaguchi O, Takeda T, Higuchi Y, Hikoso S, Taniike M, Omiya S, Mizote I, Matsumura Y, Asahi $\mathrm{M}$, Nishida K, Hori M, Mizushima N and Otsu K. The role of autophagy in cardiomyocytes in the basal state and in response to hemodynamic stress. Nat Med. 2007;13:619-24.

44. Oka T, Hikoso S, Yamaguchi O, Taneike M, Takeda T, Tamai T, Oyabu J, Murakawa T, Nakayama H, Nishida K, Akira S, Yamamoto A, Komuro I and Otsu K. Mitochondrial DNA that escapes from autophagy causes inflammation and heart failure. Nature. 2012;485:251-5.

45. Goldberg AD, Becker LC, Bonsall R, Cohen JD, Ketterer MW, Kaufman PG, Krantz DS, Light KC, McMahon RP, Noreuil T, Pepine CJ, Raczynski J, Stone PH, Strother D, Taylor H and Sheps DS. Ischemic, hemodynamic, and neurohormonal responses to mental and exercise stress. Experience from the Psychophysiological Investigations of Myocardial Ischemia Study (PIMI). Circulation. 1996;94:2402-9.

46. Gleeson M. Immune function in sport and exercise. J Appl Physiol (1985). 2007;103:693-9.

47. Heineke J and Molkentin JD. Regulation of cardiac hypertrophy by intracellular signalling pathways. Nat Rev Mol Cell Biol. 2006; 7:589-600.

48. Verreck FA, de Boer T, Langenberg DM, van der Zanden L and Ottenhoff TH. Phenotypic and functional profiling of human proinflammatory type-1 and anti-inflammatory type-2 macrophages in response to microbial antigens and IFN-gamma- and CD40L-mediated costimulation. J Leukoc Biol. 2006;79:285-93.

49. Le $Y$, Zhou $Y$, Iribarren $P$ and Wang J. Chemokines and chemokine receptors: their manifold roles in homeostasis and disease. Cell Mol Immunol. 2004;1:95-104.

50. Latronico MV and Condorelli G. MicroRNAs and cardiac pathology. Nature reviews Cardiology. 2009;6:419-29.

51. Burchfield JS, Xie M and Hill JA. Pathological ventricular remodeling: mechanisms: part 1 of 2. Circulation. 2013;128:388-400.

52. Corsten MF, Papageorgiou A, Verhesen W, Carai P, Lindow M, Obad S, Summer G, Coort SL, Hazebroek M, van Leeuwen R, Gijbels MJ, Wijnands E, Biessen EA, De Winther MP, Stassen FR, Carmeliet P, Kauppinen S, Schroen B and Heymans S. MicroRNA profiling identifies microRNA-155 as an adverse mediator of cardiac injury and dysfunction during acute viral myocarditis. Circulation research. 2012;111:415-25.

53. Zhu X, Wang H, Liu F, Chen L, Luo W, Su P, Li W, Yu L, Yang X and Cai J. Identification of micro-RNA networks in end-stage heart failure because of dilated cardiomyopathy. J Cell Mol Med. 2013;17:1173-87. 


\section{Summary}

Heart failure (HF) is the incapability of the heart to supply the body with sufficient levels of oxygenated blood. With an increasing number of patients, HF is currently one the biggest healthcare challenges worldwide. The pathophysiology of HF is marked by structural changes in the heart such as the enlargement of cardiac myocytes (hypertrophy) and production of excessive collagen (fibrosis) in the heart. Inflammation has been shown to play a crucial role in the initiation of these processes. The goal of this thesis was to increase our understanding of the inflammatory response during HF and to explore how it can be manipulated in a way that it preserves its crucial role in beneficial processes such as wound healing, debris clearance and pathogen clearance, but that its destructive effects on the cardiac tissue are diminished. The research in this thesis is described in four experimental chapters and creates several openings on how to manipulate the inflammatory response during HF.

A common cause of HF is cardiac pressure overload. This is often induced by hypertension. Chapter two focuses on the deletion and the genetic manipulation of the pro-inflammatory CD40 receptor in an animal model of angiotensin-II induced hypertension. CD40 is a receptor expressed on the cell surface of several types of inflammatory cells. The CD40 receptor was shown to be a critical regulator of the immune response in different chronic inflammatory diseases like atherosclerosis, multiple sclerosis and arthritis. In all these pathologies blocking of CD40 interactions was found to reduce disease severity. The effect of this receptor on the development of HF and cardiac hypertrophy, as induced by chronic hypertension, was never investigated before. Here we show that CD40 signalling modulates cardiac hypertrophy and we demonstrated, using mice with a mutated CD40 receptor, that the intracellular TRAF signalling molecules are involved in this mechanism. Moreover, we provided additional evidence for the importance of myeloid cells in the development of cardiac hypertrophy. These results indicate that the CD40 receptor and its interaction partners offer opportunities for the development of treatment strategies against HF.

Myocarditis is an acute inflammation of the myocardium, which is often caused by a viral infection and is a common cause for sudden cardiac death in young adults. The infiltration of inflammatory cells into the myocardium represents a hallmark of the disease. In chapter three we investigated how this acute and fulminant inflammatory response affects cardiac metabolism. For this study we used cardiac samples of viral myocarditis patients and a mouse model of virus-induced myocarditis. The acute effects of pronounced cardiac inflammation, and viral myocarditis in particular, on cardiac metabolism were not well studied so far.

We elaborately describe how the metabolism of the heart changes during this acute inflammation. A healthy cardiomyocyte generates its energy mainly by burning (oxidation) fatty acids. We observed that cardiomyocytes switch to the use of glucose instead of fatty acids as a substrate during this acute inflammation. This, in turn, leads to an inefficient energy supply. On top of this, we observed that the activity of the oxidative mechanism is reduced which further disturbs the energy supply of the cardiomyocytes. In conclusion, from our study the concept arises that cytokines, including TNF- $\alpha$, released from inflammatory cells infiltrating the myocardium, lead to the activation of NF-KB in cardiomyocytes, ultimately resulting in impairment of mitochondrial oxidative metabolism. These metabolic 
perturbations are likely to contribute to the cardiac contractile dysfunction during acute viral myocarditis.

Over the last decade, the way in which new molecules for potential therapies are identified has changed substantially. Where previously a specific hypothesis formed the basis of a new study, it is nowadays more common to work with large data sets and select interesting new molecules based on so-called high-throughput screens. In these screens a large number of different molecules can be tested on their ability to change the properties of cultured cells. The experimental work and the analysis are fully automated in these screens in order to limit the variation in the results.

In chapter four an in vitro screening approach is applied in which 194 microRNAs, previously identified to be differentially expressed in inflammatory diseases of the heart, were tested for their effects on hypertrophy, fibrosis and inflammation in different cell types. We have designed and performed this screen at a specialised company, Cenix BioScience in Dresden, Germany. These three pathophysiological processes have been studied in three relevant primary cell types. To study the effects of these microRNAs on the hypertrophic response we used primary cardiomyocytes, to determine the effect on collagen production we used primary fibroblasts and to study the effects of these microRNAs on the inflammatory response we have used macrophages that were cultured from the bone marrow of mice. By transfecting microRNAs into these cells in both unstimulated and stimulated conditions, we could quantify the role of these different microRNAs in cardiac hypertrophy, fibrosis and inflammation. Thereby, we could not only confirm roles of wellknown microRNAs, but also identify novel roles of microRNAs in HF development.

In preclinical HF research there are several commonly used rodent models of cardiac pressure overload that mimic hypertension or aortic stenosis in humans. The last decades a plethora of studies focusing on the genetic manipulation of mice in these models has been published. In chapter five, we have made a comprehensive overview of these studies. Thereby focussing on the role of inflammation during pressure overload of the heart. To this end, we have selected studies that intervened in one of the pathophysiological processes of HF. For the analyses in this chapter we extracted the data from 284 articles and combined them into a large dataset. Subsequently, this dataset was used to compare features, and to define reference values of the most commonly used preclinical pressure overload models. By analysing the phenotypes of these models we could show that aortic constriction induces more pronounced cardiac hypertrophy than angiotensin-II infusion.

Next, we used this dataset to identify novel microRNA targets for HF therapy. As the genetic manipulation of specific genes implicated in hypertrophy, fibrosis or inflammation forms the core of most studies in our dataset, the reported effect these genetic manipulations have on HF-related processes was used to build a gene-to-phenotype interaction network (GPIN). As microRNAs are established negative regulators of gene expression and have been shown to regulate pathophysiological processes associated with HF, we extended the GPIN with microRNA targeting data. This allowed the identification of microRNAs influencing cardiac inflammation, hypertrophy and/or fibrosis. Some of these microRNAs are already known in the cardiovascular field, while others have not been linked to cardiac disease previously. Accordingly, this network analysis can be used as a starting point for future preclinical studies specifically addressing the role of these microRNAs in HF. We present a novel approach to build and mine a gene to phenotype interaction network 
based on a meta-analysis. Our meta-analysis-based network analysis approach enables us to generate new insights from published cardiovascular research and the newly created methodology will also be of value for other areas of research.

The research presented in this thesis shows that inflammatory processes significantly contribute to the worsening of the disease phenotype in different aetiologies of $\mathrm{HF}$ and created several openings to manipulate this response, for example by targeting the CD40 receptor and its interaction partners or through the delivery of microRNAs. Despite these promising new findings, future translational research is needed to further confirm the potential of these results. 


\section{Samenvatting}

Hartfalen is een aandoening waarbij het hart niet meer in staat is voldoende zuurstofrijk bloed rond te pompen om in de behoeftes van het lichaam te voorzien. Het is het eindstadium van vele cardiovasculaire aandoeningen. Met een alsmaar toenemend aantal patiënten vormt hartfalen één van de grote uitdagingen voor de gezondheidszorg wereldwijd. Het doel van dit proefschrift is de rol van ontsteking (inflammatie) in het ontstaan en het verloop van hartfalen beter te begrijpen. Daarnaast willen we onderzoeken op welke manier de inflammatoire respons kan worden gemanipuleerd zodat de goedaardige onderdelen van dit proces intact blijven, zoals wondherstel en het opruimen van dode cellen en ziekteverwekkers, terwijl de schadelijke effecten op het hartweefsel worden verminderd. Het onderzoek beschreven in dit proefschrift bevat vier experimentele hoofdstukken waarin dit doel is nagestreefd.

Een veel voorkomende oorzaak van hartfalen is drukoverbelasting van het hart, vaak wordt dit veroorzaakt door een te hoge bloeddruk (hypertensie). Hoofdstuk twee van dit proefschrift beschrijft onderzoek in muizen waarbij de pro-inflammatoire CD40 receptor is weggehaald of de signaalfunctie ervan is aangepast in een diermodel waarbij hypertensie is geïnduceerd door de muizen te behandelen met angiotensine-II, dat een bloeddruk verhogende werking heeft. De CD40 receptor is aanwezig op de buitenkant van bepaalde witte bloedcellen en vervult een belangrijke functie bij het opstarten van een inflammatoire respons. Het is reeds aangetoond dat deze receptor een cruciale rol speelt bij het ontstaan van inflammatoire aandoeningen zoals atherosclerose, multipele sclerose en reumatische artritis. Bij al deze aandoeningen heeft het blokkeren van de CD40 receptor geleid tot verbeteringen in het ziektebeeld. Of de CD40 receptor ook een rol speelt bij de inflammatoire processen tijdens hartfalen was echter nog niet eerder onderzocht. In hoofdstuk twee van dit proefschrift laten we zien dat de CD40 receptor een rol speelt bij het ontstaan van een pathologische toename in grootte van de hartspier, ook wel cardiale hypertrofie genoemd. Verder laten we zien dat witte bloedcellen een cruciale rol spelen in dit mechanisme en dat de zogenaamde TRAF-moleculen belangrijk zijn in het signaleringsmechanisme binnen in de cel. Dit betekent dat de CD40 receptor en zijn interactie partners een goed aanknopingspunt bieden voor het ontwikkelen van nieuwe medicijnen tegen hartfalen.

Myocarditis is een acute ontsteking van de hartspier die vaak wordt veroorzaakt door een virusinfectie, maar ook een bacteriële infectie of bepaalde parasieten kunnen deze ziekte initiëren. Het treft vooral jonge gezonde mensen. In het derde hoofdstuk van dit proefschrift hebben we onderzocht hoe zo'n hevige acute ontsteking aan de hartspier (myocarditis) het metabolisme van het hart beïnvloedt. Daarvoor hebben we gebruik gemaakt van patiëntmateriaal en muizen die we hebben geïnfecteerd met een virus wat myocarditis veroorzaakt. De acute effecten van een hevige ontsteking van de hartspier op het cardiale-metabolisme waren tot op heden nog nooit bestudeerd.

We beschrijven op een uitgebreide manier hoe het metabolisme van het hart tijdens acute ontsteking veranderd. Een gezonde hartspiercel genereert zijn energie grotendeels door verbranding (oxidatie) van vetzuren, maar tijdens de acute ontsteking schakelt deze cel over op het gebruik van glucose i.p.v. vetzuren als substraat. Daarnaast is de activiteit van het oxidatieve mechanisme verminderd. Dit alles leidt tot een minder efficiënte 
energievoorziening voor de hartspier. Het belangrijkste concept beschreven in hoofdstuk drie is dat cytokines, afgegeven door infiltrerende immuuncellen in het hart, bijdragen aan de activatie van de pro-inflammatoire transcriptie factor NFKB in hartspiercellen. Dit leidt uiteindelijk tot verhindering van het oxidatieve mechanisme in de mitochondriën van deze cellen. Het is zeer waarschijnlijk dat deze verhindering bijdraagt aan de vermindering van de contractie van het hart tijdens acute virale myocarditis.

De manier waarop nieuwe moleculen voor mogelijke therapieën tegen hartfalen kunnen worden geïdentificeerd is erg veranderd de laatste jaren. Waar vroeger vaak een specifieke hypothese aan de basis stond van een onderzoek, wordt tegenwoordig met grote datasets gewerkt en kunnen interessante nieuwe moleculen op basis van zogenaamde highthroughput screens worden geselecteerd. In deze screens kan van een groot aantal verschillende moleculen worden getest of ze bepaalde eigenschappen van gekweekte cellen kunnen beïnvloeden. In hoofdstuk vier is een dergelijke screen beschreven die door ons is uitgevoerd bij het bedrijf Cenix BioScience in Dresden, Duitsland. In deze screen hebben we gebruik gemaakt van microRNAs. Dit zijn kleine RNA-moleculen die de expressie van hun target genen kunnen verminderen. Voor deze studie hebben we 194 microRNAs op voorhand geïdentificeerd op basis van differentiële expressie in het hart tijdens inflammatoire hartziekten. De opzet van deze screen was om te kijken wat de effecten van deze microRNAs zijn op drie cruciale processen betrokken bij het ontstaan van hartfalen, namelijk: hypertrofie (toename in grootte van hartspiercellen), fibrose (overmatige productie van bindweefsel) en inflammatie. Deze drie pathofysiologische processen zijn onderzocht in verschillende relevante celtypen die we direct uit weefsel hebben geïsoleerd. Zo hebben we hartspiercellen gebruikt om het effect van deze microRNAs op hypertrofie te bekijken en fibroblasten om productie van bindweefsel te bestuderen. Voor het bestuderen van de effecten van microRNAs op inflammatoire processen hebben we macrofagen opgekweekt uit het beenmerg van muizen. Door deze microRNAs middels transfectie, in ongestimuleerde en gestimuleerde cardiomyocyten, fibroblasten en macrofagen te brengen hebben we de rol van deze verschillende microRNAs in cardiale hypertrofie, fibrose en inflammatie in kaart gebracht. Daarbij hebben we niet alleen bestaande functies van eerder beschreven microRNAs kunnen bevestigen, maar ook nieuwe microRNAs kunnen identificeren die een rol in hartfalen spelen.

In hoofdstuk vijf, ten slotte, hebben we een uitgebreide analyse gemaakt van dierstudies waarin is gekeken naar inflammatie tijdens drukoverbelasting van het hart. Hiervoor hebben we studies geselecteerd waarbij werd ingegrepen in een van de pathofysiologische processen tijdens hartfalen. Voor de analyses in dit hoofdstuk hebben we uit 284 wetenschappelijke artikelen alle data gehaald en deze gecombineerd tot één grote dataset. Deze dataset hebben we vervolgens gebruikt om het effect van een verandering in één van de pathofysiologische processen op de andere te bekijken, een belangrijke conclusie hieruit was dat de mate van inflammatie sterk correleert met de mate van hypertrofie en fibrose.

Daarnaast hebben we met behulp van de dataset verschillende eigenschappen van deze diermodellen beter gedefinieerd en met elkaar vergeleken. Een interessante uitkomst daarbij was dat de chirurgische techniek van het afbinden van de aorta een sterkere hypertrofie induceert dan de farmacologische techniek waarbij muizen worden blootgesteld aan angiotensine II. 
Vervolgens hebben we de dataset gebruikt om fenotypische gegevens en genen met elkaar te verbinden middels een netwerkanalyse, ook wel een Gene-to-Phenotype-Network (GPIN) genoemd. Vervolgens hebben we microRNA target informatie aan dit netwerk toegevoegd zodat we per microRNA een geaccumuleerd effect op de ziekteprocessen van hartfalen hebben kunnen berekenen. Hiermee hebben we kunnen voorspellen wat voor effect elke microRNA heeft op de processen van hypertrofie, fibrose of inflammatie. Daaruit kwam naar voren dat micro-RNA-466l een zeer sterk effect had op genen die vervolgens deze drie processen kunnen beïnvloeden. Het is daarom zeer de moeite waard deze en andere door ons geïdentificeerde microRNAs nader te onderzoeken.

Het onderzoek gepresenteerd in dit proefschrift laat zien dat inflammatoire processen in belangrijke mate bijdragen aan het verergeren van het ziektebeeld bij verschillende ziekteprocessen die tot hartfalen kunnen leiden. Daarnaast heeft het geleid tot een beter begrip van de inflammatoire respons en verschillende mogelijkheden gecreëerd om deze respons te manipuleren, voornamelijk via microRNAs. Ondanks deze hoopvolle nieuwe bevindingen is er nog veel toekomstig onderzoek nodig om het potentieel van de door ons geïdentificeerde microRNAs verder te bevestigen. 


\section{Valorization}

\section{Relevance}

Heart failure (HF) is the end stage of many cardiovascular diseases. It is accompanied by severe symptoms that decrease the patients' quality of life. Half of the patients diagnosed with HF die within five years. Patient numbers have increased in the recent years, mainly because of the aging population and the increasing number of people with obesity. At this time, a targeted therapy or treatment with drugs is still lacking, therefore, the development of novel treatment strategies is an absolute necessity. For this, a better understanding of the pathology of this disease is crucial and that is exactly what the research described in this thesis has focused on.

In recent years it has become increasingly evident that HF is associated with increased inflammation in the heart, and there is increasing evidence that inflammatory factors play an active role in the pathology of many cardiovascular diseases. We have provided clues for the development of new drugs that interfere with the inflammatory response of the hart. In particular, we have shown that the CD40 receptor and its binding partners offer interesting opportunities for this and we could identify microRNAs with important roles in the pathology of HF.

We have also shown that inflammation plays a major role in the development of cardiac hypertrophy and demonstrated that severe acute inflammation interferes with the metabolism of the cardiomyocytes.

\section{Target groups}

The research described in this thesis can be of use for different groups outside the academic world. The identification of microRNAs as potential targets could be used to develop drugs for HF therapy by the pharmaceutical industry. In addition, the method of selection of microRNAs that we developed in chapter five can be applied more widely and possibly microRNAs can be selected for further in vivo studies based on our data. The microRNA research field grows rapidly and the delivery of microRNA mimics now offers exciting therapeutic possibilities. However, it is important to map the precise effects of all these microRNAs, including all of the pathophysiological processes taking place in the failing heart as we did here.

The results generated in this thesis are only of indirect value to patients. Despite the fact that many of the findings in this thesis point to a role of inflammation in HF it is difficult to translate these results into medical practice. Because the animal models used do not directly represent the clinical practice additional translational research approaches must be initiated.

\section{Activities, Products \& Innovation}

Several of the microRNAs identified by us in vitro or in silico could be used for the development of new drugs for HF therapy. The results of the screen can be used outside the cardiology field. For example, to study muscle growth or fibrosis in other organs such as kidney or lung. Our methodology of microRNA identification through network analysis can be applied in a much wider context. Therefore, we have made the source of our methodology publicly available. Direct translation of our results into products is difficult to predict at this time. We do offer our expertise in setting up a network analysis based on a meta-analysis. 


\section{Planning \& Realisation}

Several difficulties could arise in the process of developing medicines based on microRNAs. Delivery of microRNA mimics in vivo remains difficult and the many target gens make side effects difficult to predict. Indeed, despite many attempts, there are very few successful microRNA based drugs developed so far. Other results we have obtained are simply too far removed from direct application. One possible problem is that the steps that are required to get our results closer to the clinical practice cannot be put in action because it requires big investments. 


\section{Valorisatie}

\section{Relevantie}

Hartfalen is het eindstadium van vele cardiovasculaire aandoeningen. De ernstige symptomen zorgen ervoor dat patiënten een verminderde kwaliteit van leven ervaren. Bijna de helft van de mensen met de diagnose ernstig hartfalen sterft binnen vijf jaar. Het aantal mensen dat lijdt aan hartfalen neemt de laatste jaren toe. Dit komt onder meer door de vergrijzende bevolking en door een toename van het aantal mensen met een ongezonde levensstijl, waaronder zwaarlijvigheid. Op dit moment ontbreekt voor hartfalen een gerichte therapie of behandeling met medicijnen, waardoor het ontwikkelen van nieuwe behandelingsstrategieën hoogst wenselijk is. Hiervoor is een beter begrip van de pathologie van dit syndroom cruciaal en dat is dan ook waar het onderzoek beschreven in dit proefschrift op gericht is.

De laatste jaren is het steeds duidelijker geworden dat hartfalen gepaard gaat met een verhoogde aanwezigheid van ontstekingsfactoren in het hart. Ook komt er steeds meer bewijs dat deze ontstekingsfactoren een actieve rol spelen in de pathologie van de vele cardiovasculaire aandoeningen die uiteindelijk tot hartfalen kunnen leiden. Met het onderzoek beschreven in dit proefschrift hebben we nieuwe aanknopingspunten geleverd voor het ontwikkelen van medicijnen die ingrijpen in de werking van deze ontstekingsmechanismen. We laten onder meer zien dat de CD40 receptor en de bijbehorende signaaleiwitten een belangrijke rol spelen bij het ontstaan van cardiale hypertrofie, suggererend dat deze eiwitten wellicht goede aangrijpingspunten voor therapie bieden. Daarnaast hebben we verschillende microRNAs kunnen identificeren die een belangrijke rol hebben in de pathologische processen die een rol spelen bij het ontstaan van hartfalen. Ook laten we zien dat hevige acute ontstekingen het metabolisme van de hartspier flink kunnen verstoren. Deze kennis is erg waardevol bij de ontwikkeling van nieuwe medicijnen tegen hartfalen.

\section{Doelgroepen}

De onderzoeksresultaten beschreven in dit proefschrift kunnen van betekenis zijn voor verschillende groepen buiten de academische wetenschap. De door ons geïdentificeerde microRNAs hebben potentie als medicijnen voor de behandeling van hartfalen. Dit is in eerste instantie interessant voor de farmaceutische industrie. Deze microRNAs kunnen worden gebruikt voor verder in vivo onderzoek, de medicijnen die daar dan hopelijk uit voort komen zijn uiteraard in het belang van de patiënten.

Het microRNA onderzoeksveld neemt snel in grootte toe en het nabootsen van microRNAs met zogenaamde mimics biedt inmiddels interessante therapeutische mogelijkheden. Daarom is het belangrijk om van alle interessante microRNAs de precieze effecten in kaart te brengen, ook op alle relevante pathofysiologische processen, zodat eventuele bijwerkingen beter kunnen worden ingeschat. Door de rol van microRNAs in verschillende pathofysiologische processen tegelijk te bestuderen hebben we hieraan bijgedragen.

Ondanks dat de vele bevindingen in dit proefschrift die op een rol van ontstekingsmechanismen in hartfalen duiden, naast de al aanwezige bewijzen, is het moeilijk om de resultaten van dit basale onderzoek op korte termijn in de medische praktijk toe te passen. 


\section{Activiteiten, Producten \& Innovatie}

De resultaten van de screen beschreven in Hoofdstuk 4 zijn ook relevant voor buiten de cardiologie, bijvoorbeeld in het onderzoek naar spiergroei (hypertrofie) of fibrose in andere organen zoals de nieren en longen. De door ons ontwikkelde methodologie, identificatie van microRNAs door middel van netwerkanalyse kan veel breder worden toegepast, zelfs buiten de medisch biologische wetenschap. Daarom is de broncode van onze methodologie openbaar beschikbaar gemaakt. Directe vertaling van onze resultaten in concrete producten is op dit moment lastig vorm te geven, hooguit het aanbieden van de expertise in het opzetten van een netwerkanalyse gebaseerd op een meta-analyse.

\section{Planning \& Realisatie}

Eventuele risico's bij het proces van ontwikkelen van medicijnen op basis van microRNAs zijn dat het nog steeds erg lastig is om de microRNA mimics op de juiste plek in het lichaam te krijgen. Ook het feit dat microRNAs vaak vele genen tegelijk kunnen reguleren maakt de kans op ongewenste bijwerkingen groot.

Mede daardoor zijn er ondanks recente pogingen nog maar weinig succesvolle medicijnen op basis van microRNAs ontwikkeld. Andere punten uit het proefschrift staan te ver af van directe toepassing. Een mogelijk probleem is dat de stappen die nodig zijn om onze resultaten dichter bij de klinische praktijk te krijgen niet kunnen/zullen worden gezet omdat het te grote investeringen vereist. 


\section{Dankwoord}

Eindelijk is het dan zover. Mijn werk van de afgelopen jaren allemaal bij elkaar in dit boekje. Dit geeft een trots gevoel en daarom wil ik hier graag een aantal mensen bedanken, in dit enige stukje proefschrift waar ik zonder wetenschappelijke kopzorgen een verhaal kan houden dat waarschijnlijk ook nog het meest gelezen zal worden. Ten eerste wil ik mijn copromotor en begeleider Marc van Bilsen bedanken. Beste Marc, vanaf dag één van mijn promotietraject ben jij degene geweest bij wie ik altijd voor hulp kon aankloppen. Geduldig heb je je door menig van mijn manuscripten versie $1.0 \mathrm{t} / \mathrm{m}$ versie veel.nogheelveelmeer heen gewerkt en ze van commentaar voorzien. Ik mag me gelukkig prijzen met een zo'n betrokken begeleider. Jij bent cruciaal geweest voor alle onderdelen van dit proefschrift, heel veel dank hiervoor.

Ook veel dank ben ik verschuldigd aan Professor Heymans. Beste Stephane, bedankt voor de kans die ik heb gekregen om in het Cardiologie lab mijn promotieonderzoek te mogen doen. Van de vele feedback en het zien van mogelijkheden heb ik veel geleerd gedurende mijn PhD tijd. Hopelijk kan ik daar in de komende jaren ook nog de vruchten van plukken. Daarnaast wil ik Esther Lutgens bedanken, vooral in het begin ook erg betrokken bij mijn onderzoek. Het is geweldig om met een zo'n enthousiaste onderzoeker samen te mogen werken. Daarnaast ook een constante aanleverlijn voor de CD40 knock-out muizen die er in mijn onderzoek door heen zijn gegaan.

De leden van de leescommissie, die de moeite hebben genomen mijn proefschrift te bekijken en op zijn waarde te beoordelen, wil ik hier ook graag bedanken: Prof. dr. C. Schalkwijk, Prof. dr. R.A. de Boer, Dr. P.A. da Costa Martins, Prof dr. F.W. Prinzen en Prof. dr. M.P.J. de Winther.

Ook belangrijk waren de vele collega's van het cardiologie lab die op allen op een directe of indirecte manier bij mijn werk betrokken zijn geweest. Ten eerste Marieke en Tim, mede AIO's van het eerste uur. Marieke, indrukwekkend om te zien hoe snel je met je medische achtergrond vertrouwd raakte met alle biologische skills, ook je verdediging was super om te zien. Je gaat het nog ver schoppen in de medische wereld en hopelijk ook nog in de wetenschap. Tim, in het begin was ik erg onder de indruk van je grondige aanpak en je parate kennis, gelukkig was je altijd bereid om iedereen te helpen waardoor we daar allemaal van mee hebben kunnen profiteren. Het was top om met je samen te werken, veel succes en geluk met je jonge gezinnetje!

Georg en Robin, Jullie waren de collega's waarmee ik het uiteindelijk het meest heb samen mogen werken en daarom zijn jullie nu ook mijn paranimfen. Georg, je was ook vanaf begin 2011 al in het lab dus precies dezelfde periode als ik. Zonder jouw bio-informatica skills was dit proefschrift, met name hoofdstuk 5 nooit tot stand gekomen. Daarnaast was je een erg gezellige collega die menig etentje en borrel mee tot het einde heeft uitgezeten! Hopelijk gaan we nog vaak eens een biertje drinken. Robin, we hebben een jaar lang samen gewerkt bij Cenix in Dresden. Met het mooie resultaat beschreven in hoofdstuk 4. Als Masterstudent maakte je al een degelijke en gedreven indruk. Ik weet zeker dat je nog veel vruchten gaat plukken van je goede werk. Hopelijk blijf je ook nog de tijd vinden voor handballen! 
Veel dank ook aan de technicians in ons lab: Rick van Leeuwen, opper-analist en voor meer dan een jaar kamergenoot, Wouter Verhesen, mosasaurus of muziek, altijd verbaasde je me met al je diverse hobby's en humor. Kevin Custers, als enige echte Sjeng in het Cardio lab maakte je op mij een onvergetelijke indruk.

Als jonge onderzoeker is het fijn als er mensen zijn bij wie je terecht kan voor vragen en advies, dan denk ik vooral aan Anna en Blanche. Anna, je zorgde ook altijd voor de goede sfeer in het lab en het eten bij het BBQ-restaurant in Boston zal ik niet gauw vergeten. Blanche ook bij jou kon ik altijd terecht met vragen, veel dank voor alles en veel succes met je eigen groepje en je gezinnetje. Maarten, op het moment dat ik begon was jij de enige overgebleven en ervaren aio van de Heymans groep. Fantastisch hoe veel ik van je heb kunnen leren en ook dat je me meteen thuis hebt laten voelen in Maastricht, dankjewel!

Niet te vergeten ook de klinische aio's, Mark $\mathbf{H}$ en Jort, door jullie verloor ik ook het klinische oogpunt niet uit het oog, bedankt ook voor de goede sfeer tijdens de koffie en vlaai momenten.

Ook de Leuvense telg van het Lab mag hier niet vergeten worden, Lucas, Ward en Sophie inmiddels zijn jullie PhDs ook ten einde en wens ik jullie veel succes in verdere carrières. Paolo, de vele hulp van jou bij mijn dierexperimenten was cruciaal. Grazie mille!

Inmiddels is er weer ook een nieuwe lichting PHD studenten begonnen in ons lab. Monika \& Steffie veel succes en bedankt voor alle gezelligheid!

Also thanks all other colleagues from the groot-cardiolab amongst them all the members of the de Windt lab: Rio, Burcu, Leonne, Ellen, Stefanos, Serve and all the others that have worked there over the last several years. Speciale dank ook naar Nicole voor alle hulp met mijn muis experimenten.

Dan zijn er ook nog een aantal studenten die hun bijdrage aan dit proefschrift hebben geleverd: Veel dank vooral aan Wendy, Feras, Rob en natuurlijk alle andere studenten die we hebben voorbij zien komen in de Heymans groep die voor goede sfeer en gezonde afwisseling hebben gezorgd.

Barbara en Lilian voor alle logistieke en administratieve ondersteuning en natuurlijk gezelligheid.

Buiten de cardiologie vakgroep zijn er ook nog UM-collega's die ik wil bedanken. Met name Alex Remels voor de goede samenwerking, waarvan de resultaten in hoofdstuk 3 beschreven staan. Bij fysiologie Chantal Munts voor alle in vitro hulp, alle mensen bij het CPV ben ik dank verschuldigd voor verzorging van mijn muizen en Erwin Wijnands voor alle hulp met de FACS-experimenten.

For over a year of my PhD time I have lived in the beautiful city of Dresden where I had the chance to work at Cenix Bioscience. Regretfully I also had to experience the sad ending of the company. Despite this misfortune my time at Cenix was one big nice adventure that learned me a lot about (1) High throughput screening (2) Working in a company compared to academia (3) What nice a city Dresden is when living in the Neustadt (4) That you can make beautiful hikes in Sächsische Schweiz (5) and I've learned a lot about the Germans. The hard work there has also yielded nice results as described in chapter four of this book.

For this I would like to thank all people at Cenix, also for giving me a warm welcome in Dresden and a great time. I should name everyone, because all of you made my stay a great and informative time. But in particular I need to thank: Birte and Kerstin for all the help in the design of the screens and the interpretation of the results. Alex, Kartini, Sarah thanks 
for all practical help and Steffen, Andrew and Christophe for all help with the data analysis, image analysis in Definiens, Statistics in KNIME and so on, it would not have been possible without all of you! Also thanks to Gisela also for helping me on the housing hunt, my Dresden home was the best apartment I ever had.

Zonder vrienden en goede afleiding is het leven nu eenmaal een stuk saaier en gave momenten zorgen ook weer voor nieuwe inspiratie en motivatie. Daarom veel dank aan al mijn Wageningse vrienden voor de vele mountainbike en racefiets tochtjes overal in ZuidLimburg en de Ardennen, ook de transalp tochten waren geweldig. Een legendarische skivakantie in Val-tho kan ik mij ook nog goed herinneren. Thanks: Karel, Thomas, Wouter, Ronnie, Robert, Maarten, Remko, Bas, Sjoerd, hopelijk komen er nog veel meer van dit soort mooie avonturen bij in de komende jaren!

Werken in het buitenland is leuk, maar vooral als je altijd een geweldige plek hebt om weer thuis te komen daarvoor veel dank aan alle vrienden uit Venray: Niels, Hanneke, Johan, Jer, Chiel, Linda, Chiel, Joris, Floor, Stan, Leonie, Hanneke, Bram, Martijn, Jasper, Joris, Elle, Joep, Linda, Jesper, Danielle, Marco, Anna. Veel geluk met jullie startende gezinnetjes en hopelijk blijft er ook nog af en toe tijd over om bier te drinken.

Mijn lieve zus en zwager, Marieke \& Nick, wil ik ook bedanken voor de vele gezellige bezoekjes aan de Zakstraat en waarbij in de zomer menig wijnvoorraad is gesneuveld op mijn dakterras.

Zonder mijn ouders, Toon \& Diny, had dit proefschrift niet tot stand kunnen komen, alleen al omdat ik zonder hen natuurlijk nooit ter wereld was gekomen, maar niet in de laatste plaats omdat ze mij altijd gesteund hebben in al mijn keuzes. Van mijn studie biologie tot dit resultaat. Dit boekje is er daarom ook voor jullie.

Lieve Judith, jou wil ik natuurlijk het meest bedanken. Je bent de allerliefste persoon die ik ooit heb ontmoet en ik mag me gelukkig prijzen met jou als vriendin. Daarnaast heb je me ook geholpen bij het tot stand komen van dit proefschrift en vaak heb je me van nuttige feedback voorzien bij het oefenen van de vele presentaties in de laatste jaren. Zelfs voor dit boekje heb je jouw spelling en grammatica skills ingezet. Hopelijk kunnen we nog lang zo door gaan. Dankjewel, je bent de allerliefste. 


\section{Biography}

Wouter Derks was born on the $5^{\text {th }}$ of May 1984 in Heide, near Venray, the Netherlands. After finishing his secondary education with profile nature, technology and health, he started to study biology at Wageningen University. In Wageningen he specialised in animal biology and immunology. He completed a thesis on the role of chemokines in the immune system of teleost fish. Later, Wouter went to perform research internships at the INRA institute near Paris and at the INSERM institute in Montpellier, France. After receiving his MSc degree from Wageningen University, Wouter commenced his PhD program in the lab of prof. dr. S. Heymans at Maastricht University, Department of Cardiology, in January 2011 in a cooperation with prof. dr. E. Lutgens and supervised by dr. M. van Bilsen.

During his PhD he studied the role of the immune response on the development of cardiac disease using pre-clinical animal models. He worked on the role of the CD40 receptor in hypertensive heart disease and studied the effect of acute inflammation in the heart induced by viral myocarditis. From February 2014 until March 2015 Wouter has worked at Cenix Bioscience $\mathrm{GmbH}$, Dresden, Germany, in the context the European CardiomiR project, there he developed high-throughput screening assays to identify microRNAs involved in inflammatory cardiac pathology.

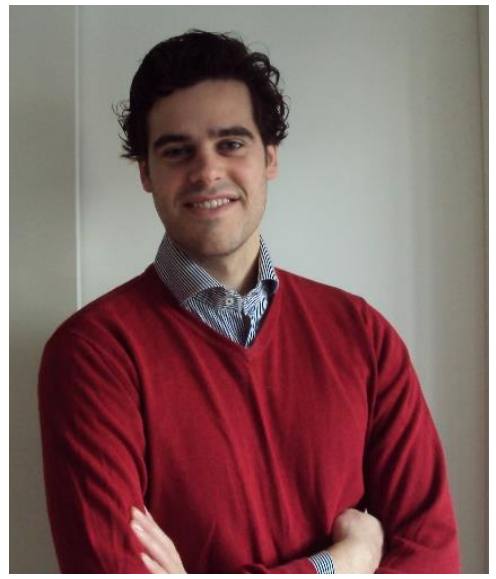




\section{List of Publications}

Submitted: Inhibition of CD40-CD40 ligand (CD40L) and downstream TRAF signalling reduces angiotensin II induced cardiac hypertrophy. Derks W, Carai P, Boon L, Lutgens E, Van Bilsen M, Heymans S.

In preparation: Inflammation-induced metabolic remodelling of the heart in acute viral myocarditis. Remels $A^{*}$, Derks $W^{*}$, Verhees K, Kelders M, Summer G, Theije C, Heymans S, Papageorgiou $A$, van Bilsen $M$

*contributed equally

In preparation: MicroRNAs act as pleiotropic regulators of heart failure progression - A high-throughput phenotypical screen in three primary cardiac cell types. Verjans $\mathrm{R}^{*}$, Derks $W^{*}$, Korn K, Sönnichsen B, Van Bilsen M, Schroen B, Heymans S.

*contributed equally

Submitted: Literature-based Gene to Phenotype Interaction Network analysis reveals novel microRNA regulators in Heart Failure. Derks $W^{*}$, Summer $G^{*}$, Coenen R, Heymans S, Van Bilsen M.

*contributed equally

Hypertension is a conditional factor for the development of cardiac hypertrophy in type 2 diabetic mice. van Bilsen $M$, Daniels A, Brouwers $O$, Janssen BJ, Derks WJA, Brouns AE, Munts C, Schalkwijk CG, van der Vusse GJ, van Nieuwenhoven FA. PLoS One. 2014 Jan 9;9(1)

Diversification of IFNp-inducible $\mathbf{C X C b}$ chemokines in cyprinid fish. van der $A a L M$, Chadzinska M, Derks W, Scheer M, Levraud JP, Boudinot P, Lidy Verburg-van Kemenade BM. Dev Comp Immunol. 2012 Oct;38(2) 